\title{
Welcome to the 10th volume of Epigenomics
}

\author{
Joseph Martin*,1 \\ ${ }^{1}$ Future Medicine Ltd, Unitec House, London, N3 1QB, UK \\ * Author for correspondence: j.martin@futuremedicine.com
}

\section{"Over the past year, we have seen some great strides in the development of epigenetics for medical applications."}

First draft submitted: 13 November 2017; Accepted for publication: 15 November 2017; Published online: 1 December 2017

Welcome to the 10th volume of Epigenomics. I would like to take this opportunity to wish all of our readers a Happy New Year. In this short Foreword, I would like to take a look back at some of the journal highlights of 2017.

Over the past year, we have seen some great strides in the development of epigenetics for medical applications. However, this advancing field, while niche, still needs to overcome a number of obstacles before we see a more widespread use of this technology. It has been a privilege for Epigenomics to publish work in this fascinating field, which continues to be of the highest quality.

\section{Special focus issues}

A particular highlight of 2017 was to see the publication of two special focus issues. These were:

- Issue 3: 'Effects of the in utero environment on the epigenome' [1];

- Issue 4: 'Epigenetics of the immune system and alterations in inflammation and autoimmunity' [2].

I would like to give special thanks to the guest editors of both special issues, Richard Saffery (University of Melbourne), Jorg Tost (CEA-Institut de Génomique), Steffen Gay (University of California) and Gary Firestein (University Hospital Zurich) without whom publication would not be possible.

Special focus issues are a great way of showcasing the latest research in a specific subfield or concept in nanomedicine. By having an entire journal issue dedicated to a topic, each article can focus on one aspect in detail, contributing to the bigger picture when they are all compiled together. I would like to thank all of the Guest Editors who have aided us in the organization of this year's special focus issues, without whom publication would not be possible.

\section{Article highlights}

We have also published a number of outstanding manuscripts in the journal this year. I would like to draw your attention to some of the particular highlights of 2017.

At the time of writing our most read article from 2017 was a review entitled 'Epigenetics and allergy: from basic mechanisms to clinical applications' by Potaczek et al. [3]. Part of our second special focus issue, the authors review key facts on the involvement of epigenetic modifications in allergic diseases and critically evaluate the lessons learned from epigenome-wide association studies.

Our second most-accessed article authored by Andrew E Teschendorff and Shijie C Zheng was a critical review of the major existing algorithms for correcting cell-type composition in the context of Illumina Infinium Methylation Beadarrays, which provided recommendations to the epigenome-wide association studies community [4].

Our most popular research article from the past year was a piece by Mayne et al. entitled 'Accelerated placental aging in early onset preeclampsia pregnancies identified by DNA methylation' [5]. The team from University of Adelaide worked to determine whether dynamic DNA methylation changes in the human placenta can be used to predict gestational age.

Altmetrics is a powerful way to measure the impact of our publications. An excellent example of this from 2017 has been the research article by Moody et al. entitled 'Postnatal diet remodels hepatic DNA methylation in metabolic pathways established by a maternal high-fat diet' [6], which received an Altmetrics score of 84 since its 


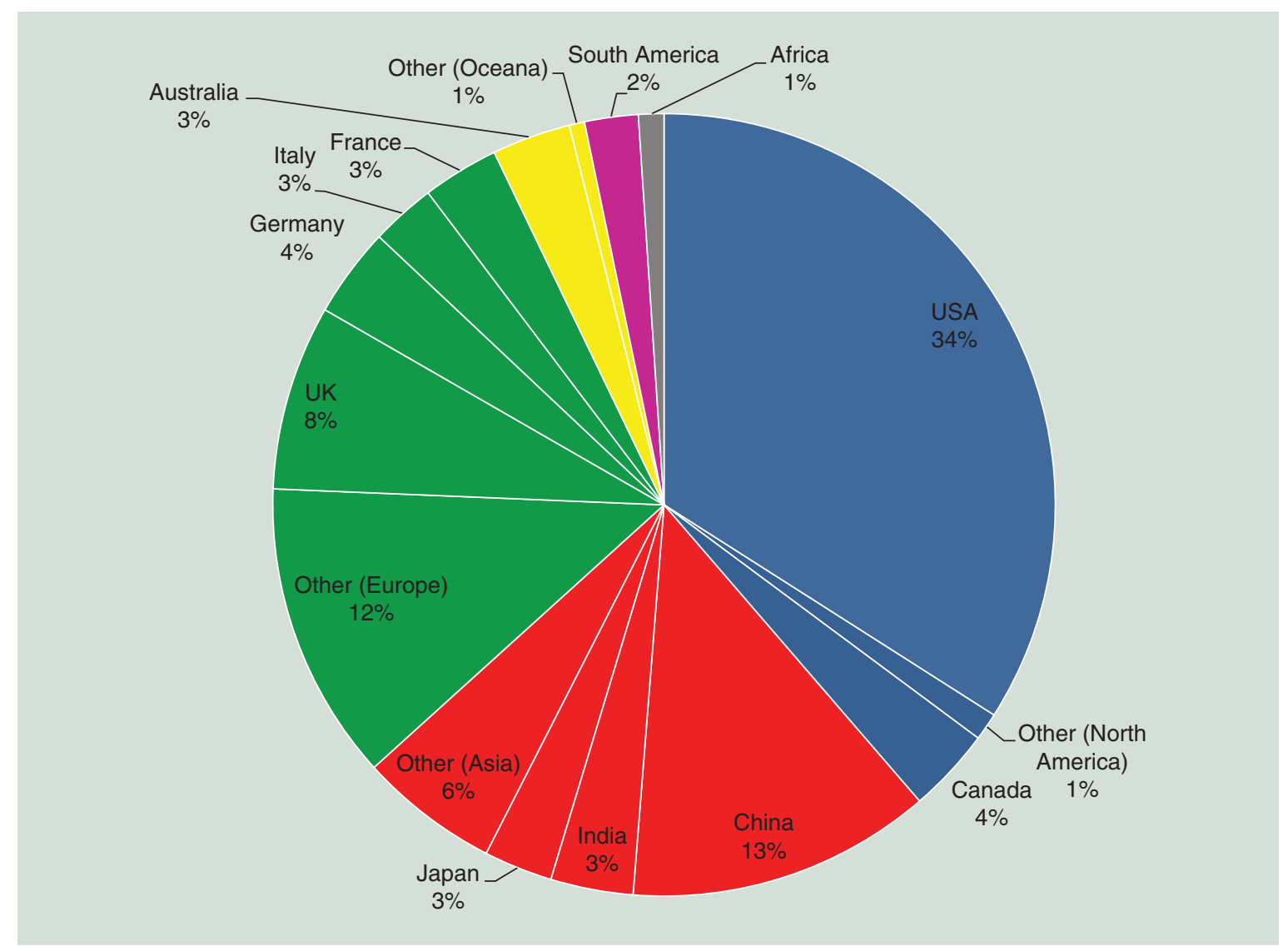

Figure 1. Proportion of readership demographics for Epigenomics in 2017.

publication in November. A combination of tweets, news outlets and blogs has helped this article reach a wide range of readers worldwide. In this study, the team from University of Illinois at Urbana-Champaign emphasizes the dynamic nature of the methylome even after early life.

\section{Geographical spread of our contributors}

As the diversity of the research community continues to grow, the readership of Epigenomics has also changed throughout the past year. Our content was most read by experts in North America (39\%), with the majority based within the USA (34\%). China also makes up a large proportion of our readership (13\%). A high proportion of our readers also come from Europe, in particular the UK (8\%), Germany (4\%), France (3\%) and Italy (3\%) (Figure 1). Epigenomics continues to welcome both readers and content from across the world.

\section{Twitter}

As many of you will be aware, Epigenomics has a Twitter account. We showcase content highlights, journal updates and key news from the field. I have thoroughly enjoyed engaging with our readers and authors on these platforms and encourage anyone on Twitter to follow us (@fsgepi) [7].

\section{Conclusion}

I would like to take this opportunity to thank everyone that has helped with the success of Epigenomics in 2017. We welcome unsolicited research, review and opinion article proposals, and would be delighted to hear from you if you are interested in submitting to the journal.

Many thanks for your continuing interest and support for Epigenomics, I look forward to collaborating with many of you over the coming year. 
Financial \& competing interests disclosure

$J$ Martin is an employee of Future Medicine Ltd. The author has no other relevant affiliations or financial involvement with any organization or entity with a financial interest in or financial conflict with the subject matter or materials discussed in the manuscript apart from those disclosed.

No writing assistance was utilized in the production of this manuscript.

\section{References}

1 Saffery R. Effects of the in utero environment on the epigenome. Epigenomics 9(3), 209-211 (2017).

2 Tost J, Gay S, Firestein G. Epigenetics of the immune system and alterations in inflammation and autoimmunity. Epigenomics 9(4), 371-373 (2017).

3 Potaczek DP, Harb H, Michel S, Alhamwe BA, Renz H, Tost J. Epigenetics and allergy: from basic mechanisms to clinical applications. Epigenomics 9(4), 539-571 (2017).

4 Teschendorff AE, Zheng SC. Cell-type deconvolution in epigenome-wide association studies: a review and recommendations. Epigenomics 9(5), 757-768 (2017).

5 Mayne BT, Leemaqz SY, Smith AK, Breen J, Roberts CT, Bianco-Miotto T. Accelerated placental aging in early onset preeclampsia pregnancies identified by DNA methylation. Epigenomics 9(3), 279-289 (2017).

6 Moody L, Chen H, Pan Y-X. Postnatal diet remodels hepatic DNA methylation in metabolic pathways established by a maternal high-fat diet. Epigenomics 9(11), 1387-1402 (2017).

7 Twitter. Epigenomics. https://twitter.com/fsgepi 
(

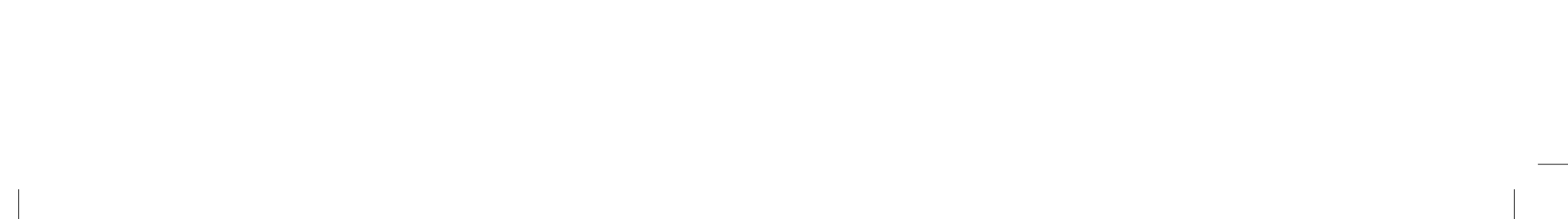




\title{
Role of MeCP2 in neurological disorders: current status and future perspectives
}

\author{
Juan Ausió* ${ }^{\prime} 1$ \\ ${ }^{1}$ Department of Biochemistry \& Microbiology, University of Victoria, Victoria, BC, V8W 3P6, Canada \\ * Author for correspondence: jausio@uvic.ca
}

\begin{abstract}
"MeCP2 has also been shown to directly participate in important neurodegenerative disorders such as Parkinson's disease, Huntington's disease and Lou Gehrig's disease and Alzheimer's."
\end{abstract}

First draft submitted: 29 September 2017; Accepted for publication: 10 October 2017; Published online: 27 November 2017

Keywords: epigenetics $\bullet$ methyl CpG binding protein $2 \bullet$ neurodevelopmental and neurodegenerative disorders $\bullet$ post-translational modifications $\bullet$ Rett syndrome $\bullet$ substance abuse

Methyl CpG protein binding 2 (MeCP2) is a protein first described as a member of a complex family of 'reader' proteins that binds to mehtylated DNA through their methyl binding domain (MBD) [1] (Figure 1). MeCP2 also has a structurally important RNA binding domain in the form of an AT-hook [2] that together with its MBD allow it bind to its nucleic acid (DNA and RNA) partners within a chromatin context [3]. In addition, it has the ability to interact with diverse protein partners [4]. Interaction with these partners is often modulated by a plethora of $\mathrm{MeCP} 2$ post-translational modifications (PTMs) [5]. All these features together are critical for the protein's normal complex functionality.

Although MeCP2 has been extensively studied within the context of Rett syndrome (RTT) [6], its dysregulation is involved in a multitude of other diseases (Figure 1) such as for instance cancer. Dysregulation of DNA methylation is an epigenetic component that plays an important role in cancer [7] and the MeCP2 gene has recently been shown to be an oncogene [8]. In the brain where $\mathrm{MeCP} 2$ is a major constituent of neuronal chromatin [3] the homeostasis of this protein is critical for the proper functioning of this organ [9]. Under normal conditions, such homeostasis is maintained within a range since the protein can experience fluctuations due to different states of activity; an example of this is provided by the change in activity levels experienced during the daily circadian cycle [10]. Thus, it is not surprising that abnormal alterations of the MeCP2 levels beyond its regular oscillatory pattern and mutations in the gene encoding this protein are involved in a large variety of neurological disorders beyond RTT [4]. Additionally, MeCP2 PTMs, such as phosphorylation, can be modified with changes in neuronal activity and can indirectly affect MeCP2 homeostasis [11]. Abnormal alterations of these PTMs are also often observed in several neurological disorders [3]. What follows is a summary of the current knowledge about how these structural and functional alterations of $\mathrm{MeCP} 2$ contribute to neurological disorders and the future perspectives.

\section{MeCP2 \& neurological disorders}

Due to its aforementioned high abundance in the brain, any kind of disturbances of MeCP2 in this tissue have the potential to be involved in all of the neurodevelopmental and neurodegenerative disorders [4] (Figure 1). Disturbances in MeCP2 levels may also result from external factors or injury such as substance abuse [3] or concussion. Moreover, MeCP2 is a critical regulator of the brain-derived neurotrophic factor ( $B d n f)$ gene [12] as well as other neuronal-specific long genes [13], which are important for the growth and differentiation of neurons and proper axon and synaptic formation. Bdnffunction itself has many connections to several of the neurological disorders described here.

Neurodevelopmental disorders

In relation to neurodevelopmental disorders, the roles of MeCP2 in RTT and in MeCP2 duplication syndrome [14] have been most extensively studied. RTT results from MeCP2 mutations in $85-90 \%$ of cases, however, this does

Future Medicine 


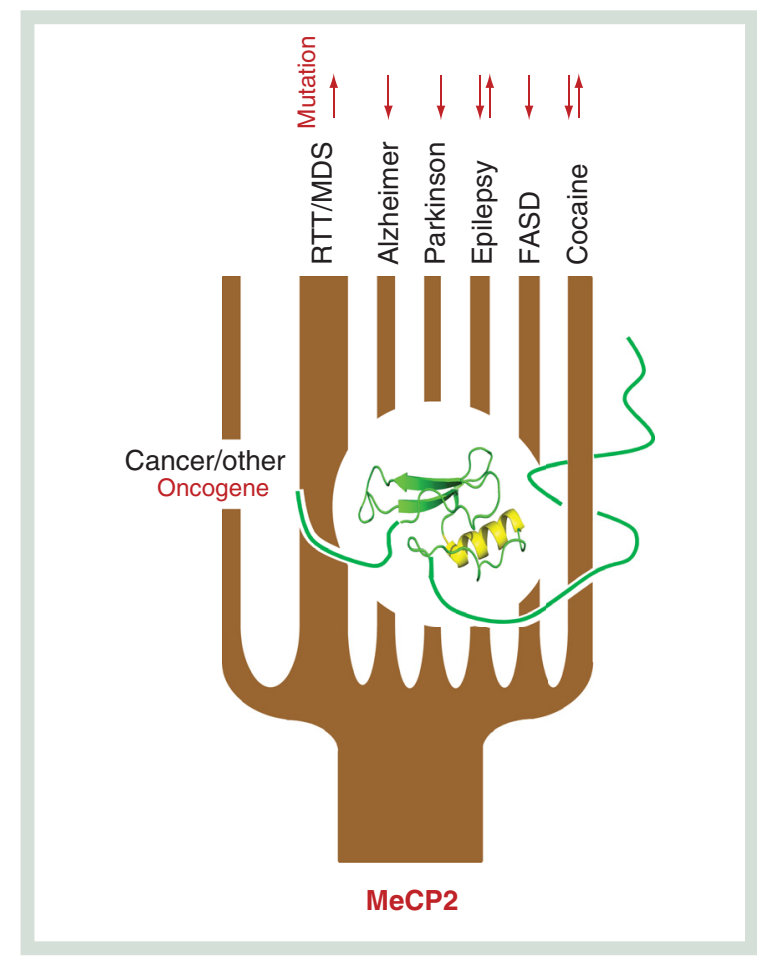

Figure 1. Schematic representation highlighting several of the many diseases involving MeCP2. The occurrence of the MeCP2 alterations resulting from mutations or its abnormal increased or decreased levels (indicated by arrows) is shown. Also shown in the center of the figure is the 3D organization of the methyl binding domain of the protein.

MeCP2: Methyl CpG protein binding 2.

not mean that all of the RTT cases are the result of MeCP2 mutation, nor that all MeCP2 mutations result in RTT [15] or only in RTT.

As a point of interest, it has recently been found that, chromatin regulators other than MeCP2 are implicated in several RTT cases [16]. Conversely, an MeCP2 (S164I) mutation was recently shown to be involved in Asperger syndrome and early-onset schizophrenia [17]. Additionally, MeCP2 AT-hook1 mutations have now been observed in patients with intellectual disability and schizophrenia [Sheikh TI, Harripaul R, Ayub M, Vincent JB. MeCP2 AT-hook1 mutations disrupt DNA binding and chromatin compaction in patients with intellectual disability and schizophrenia. Human Mutation (2017); submitted].

Diseases that are also associated with MeCP2 irregularities but have not been as extensively researched within this section are: Down syndrome, attention deficit hyperactivity disorder, mental retardation and schizophrenia [4]. In the first three instances MeCP2 appears to be under expressed [4] (Figure 1). In schizophrenia, however, MeCP2 appears to upregulate the expression of certain GABAergic neuronal genes that in some cases maybe directly involved in this disease in addition to the involvement of the previously described MeCP2 mutations [17][SHEIKH TI, HARRIPAUL R, AYUB M, VINCENT JB. MECP2 AT-HOOKI MUTATIONS DISRUPT DNA BINDING AND CHROMATIN COMPACTION IN PATIENTS WITH INTELLECTUAL DISABILITY AND SCHIZOPHRENIA. HUMAN MUTATION (2OI7); SUBMITTED].

\section{Neurodegenerative disorders \& brain alterations}

$\mathrm{MeCP} 2$ has also been shown to directly participate in important neurodegenerative disorders such as Parkinson's disease, Huntington's disease and Lou Gehrig's disease and Alzheimer's. [4]. Of note, early life exposure of mice to lead $(\mathrm{Pb})$, which results in a latent increase in Alzheimer's, has been recently shown to significantly decrease the cerebral levels of MeCP2 in these organisms [18].

In addition to all this, MeCP2 has been shown to participate in many other brain disorders such as epilepsy, bipolar mood disorder, depression and substance abuse (cocaine, fetal alcohol syndrome) [4]. Most of these result in serious and lifelong physical, mental and behavioral difficulties and are an enormous financial burden to society, yet the role of MeCP2 has not been extensively studied and hence its role in these brain alterations is not well understood. 


\section{MeCP2 as an epigenetic marker with high potential for treatment development of neurological disorders}

Besides its ability to act as an epigenetic 'reader' of DNA methylation, MeCP2 can recruit chromatin regulatory complexes such as NCoR/SMRT (nuclear receptor corepressor 2/silencing mediator for retinoid or thyroidhormone receptors) which contain histone deacetylases [19]. Therefore, MeCP2 can also operate as a 'writer' contributing to modifications of the epigenetic chromatin landscape. Thus, like with any other epigenetic factors, the effects of MeCP2 should be amenable to reversal hence providing a good therapeutic target for neurological disorders where the protein is involved [20]. Indeed, it should be possible to use MeCP2 druggable regulators as those recently identified using an RNA interference screen procedure [21]. Moreover, we have shown that the histone deacetylase inhibitors sodium butyrate and trichostatin A can decrease the levels of MeCP2 [22]. This potentially opens a door to the use of these inhibitors in the treatment of neurological disorders similarly to the way that they are routinely used in cancer treatment [23].

\section{Conclusion \& future perspective}

As described in the previous section, more emphasis should be put in the participation of MeCP2 in normal and altered states of the brain beyond RTT and MeCP2 duplication syndrome. Similarly, at the molecular level more attention needs to be paid to the role of the potentially detrimental effects arising from the disturbances of the $\mathrm{MeCP} 2$ interactions with RNA. Research on this front should not only focus on RNA splicing [24], but also in the participation of MeCP2 in its direct interactions with RNA such as those recently described for lncRNA [25] and its putative interaction with methylated m6A RNA [26], which remain relatively unexplored territory. We are still far away from our complete understanding all the details on the molecular biology of this highly complex multifunctional protein in its normal physiological setting. This might provide newly unexpected and exciting results and is key to ultimately understand the functional implications of MeCP2 in disease.

From the information already available, several questions arise: do the disturbances in neuronal activity resulting from neurological disorders affect MeCP2 PTMs other than histone phosphorylation? And if so, what are the mechanisms involved? There is also not much known about the role of MeCP2 in the brain alterations resulting from physical injury such as concussion, a prevalent sports injury. To what extent is MeCP2 also involved in these?

Despite the clear involvement of MeCP2 in any brain alteration, a quick pubmed search of recent papers on the topic of this article reveals that $90 \%$ of the current MeCP2research is related to RTT. This underscores a gap between what is and what could be researched and shows the much needed expansion of research on this topic.

Acknowledgements

The author is thankful to HMO Reid and ME Freeman for carefully proofreading the manuscript.

Financial \& competing interests disclosure.

Preparation of this manuscript was supported by the Canadian Institutes of Health Research (CIHR grant MOP -130417). The author has no other relevant affiliations or financial involvement with any organization or entity with a financial interest in or financial conflict with the subject matter or materials discussed in the manuscript apart from those disclosed.

No writing assistance was utilized in the production of this manuscript.

\section{References}

1. Fatemi M, Wade PA. MBD family proteins: reading the epigenetic code. J. Cell Sci. 119(Pt 15), 3033-3037 (2006).

2. Filarsky M, Zillner K, Araya I et al. The extended AT-hook is a novel RNA binding motif. RNA Biol. 12(8), 864-876 (2015).

3. Ausio J. MeCP2 and the enigmatic organization of brain chromatin. Implications for depression and cocaine addiction. Clin. Epigenetics 8, 58 (2016).

4. Ausió J, De Paz A, Esteller M. MeCP2: the long trip from a chromatin protein to neurological disorders. Trends Mol. Med. 20(9), 487-498 (2014).

5. Bellini E, Pavesi G, Barbiero I et al. MeCP2 post-translational modifications: a mechanism to control its involvement in synaptic plasticity and homeostasis? Front. Cell Neurosci. 8, 236 (2014).

6. Zoghbi HY. Rett syndrome and the ongoing legacy of close clinical observation. Cell 167(2), 293-297 (2016).

7. Baylin SB, Jones PA. Epigenetic determinants of cancer. Cold Spring Harb. Perspect. Biol. 8(9), pii:a019505 (2016).

8. Neupane M, Clark AP, Landini S et al. MECP2 is a frequently amplified oncogene with a novel epigenetic mechanism that mimics the role of activated RAS in malignancy. Cancer Discov. 6(1), 45-58 (2015). 
9. Collins AL, Levenson JM, Vilaythong AP et al. Mild overexpression of MeCP2 causes a progressive neurological disorder in mice. Hum. Mol. Genet. 13(21), 2679-2689 (2004).

10. Martinez De Paz A, Vicente Sanchez-Mut J, Samitier-Marti M et al. Circadian cycle-dependent MeCP2 and brain chromatin changes. PLoS ONE 10(4), e0123693 (2015).

11. Thambirajah AA, Eubanks JH, Ausio J. MeCP2 post-translational regulation through PEST domains: two novel hypotheses: potential relevance and implications for Rett syndrome. Bioessays 31(5), 561-569 (2009).

12. Huang EJ, Reichardt LF. Neurotrophins: roles in neuronal development and function. Annu. Rev. Neurosci. 24, 677-736 (2001).

13. Gabel HW, Kinde B, Stroud H et al. Disruption of DNA-methylation-dependent long gene repression in Rett syndrome. Nature 522(7554), 89-93 (2015).

14. Van Esch H, Bauters M, Ignatius J et al. Duplication of the MECP2 region is a frequent cause of severe mental retardation and progressive neurological symptoms in males. Am. J. Hum. Genet. 77(3), 442-453 (2005).

15. Suter B, Treadwell-Deering D, Zoghbi HY, Glaze DG, Neul JL. Brief report: MECP2 mutations in people without Rett syndrome. J. Autism Dev. Disord. 44(3), 703-711 (2014).

16. Sajan SA, Jhangiani SN, Muzny DM et al. Enrichment of mutations in chromatin regulators in people with Rett syndrome lacking mutations in MECP2. Genet. Med. 19(1), 13-19 (2017).

17. Curie A, Lesca G, Bussy G et al. Asperger syndrome and early-onset schizophrenia associated with a novel MECP2 deleterious missense variant. Psychiatr. Genet. 27(3), 105-109 (2017).

18. Eid A, Bihaqi SW, Renehan WE, Zawia NH. Developmental lead exposure and lifespan alterations in epigenetic regulators and their correspondence to biomarkers of Alzheimer's disease. Alzheimers Dement. (Amst) 2, 123-131 (2016).

19. Lyst MJ, Ekiert R, Ebert DH et al. Rett syndrome mutations abolish the interaction of MeCP2 with the NCoR/SMRT co-repressor. Nat. Neurosci. 16(7), 898-902 (2013).

20. Shah RR, Bird AP. MeCP2 mutations: progress towards understanding and treating Rett syndrome. Genome Med. 9 (1), 17 (2017).

21. Lombardi LM, Zaghlula M, Sztainberg Y et al. An RNA interference screen identifies druggable regulators of MeCP2 stability. Sci. Transl. Med. 9(404), (2017).

22. Good KV, Martinez De Paz A, Tyagi M et al. Trichostatin A decreases the levels of MeCP2 expression and phosphorylation and increases its chromatin binding. Epigenetics doi: 10.1080/15592294.2017.1380760 (2017) (Epub ahead of print).

23. Johnstone RW. Histone-deacetylase inhibitors: novel drugs for the treatment of cancer. Nat. Rev. Drug Discov. 1(4), 287-299 (2002).

24. Young JI, Hong EP, Castle JC et al. Regulation of RNA splicing by the methylation-dependent transcriptional repressor methyl-CpG binding protein 2. Proc. Natl Acad. Sci. USA 102(49), 17551-17558 (2005).

25. Maxwell SS, Pelka GJ, Tam PP, El-Osta A. Chromatin context and ncRNA highlight targets of MeCP2 in brain. RNA Biol. 10(11), 1741-1757 (2013).

26. Roignant JY, Soller M. m6A in mRNA: an ancient mechanism for fine-tuning gene expression. Trends Genet. 33(6), 380-390 (2017). 


\title{
Epigenetic profiles in polyglutamine disorders
}

\author{
Hongmei Liu*, ${ }^{*}$, Tie-Shan Tang ${ }^{1}$ \& Caixia Guo*,2 \\ ${ }^{1}$ State Key Laboratory of Membrane Biology, Institute of Zoology, University of Chinese Academy of Sciences, Chinese Academy of \\ Sciences, Beijing 100101, China \\ ${ }^{2}$ CAS Key Laboratory of Genomics \& Precision Medicine, Beijing Institute of Genomics, University of Chinese Academy of Sciences, \\ Chinese Academy of Sciences, Beijing 100101, China \\ * Author for correspondence: Tel.: +86 106480 7281; liuhongmei@ioz.ac.cn; guocx@big.ac.cn
}

The dominant polyglutamine (polyQ) disorders are a group of progressive and incurable neurodegenerative disorders, which are caused by unstable expanded CAG trinucleotide repeats in the coding regions of their respective causative genes. The most prevalent polyQ disorders worldwide are Huntington's disease and spinocerebellar ataxia type 3. Epigenetic mechanisms, such as DNA methylation, histone modifications and chromatin remodeling and noncoding RNA regulation, regulate gene expression or genome function. Epigenetic dysregulation has been suggested to play a pivotal role in the pathogenesis of polyQ disorders. Here, we summarize the current knowledge of epigenetic changes present in several representative polyQ disorders and discuss the potentiality of miRNAs as therapeutic targets for the clinic therapy of these disorders.

First draft submitted: 20 July 2017; Accepted for publication: 28 September 2017; Published online: 27 November 2017

Keywords: epigenetic dysregulation • pathogenesis • polyglutamine disorders

\section{Background}

Polyglutamine disorders

Polyglutamine (PolyQ) disorders constitute a group of at least nine hereditary neurodegenerative disorders known to date, including Huntington's disease (HD), spinal and bulbar muscular atrophy, dentatorubral-pallidoluysian atrophy, spinocerebellar ataxia (SCA) type 1, 2, 3, 6, 7 and 17 [1-3]. All polyQ disorders are autosomal dominantly inherited disorders except spinal and bulbar muscular atrophy, which is sex dependent. They are caused by unstable expanded CAG trinucleotide repeats in the coding regions of their respective causative genes, leading to mutant proteins with an abnormally long stretch of repeated glutamines (Table 1).

The most common type of polyQ disorders worldwide is HD, with a prevalence of 10.6-13.7/100,000 in the west [4]. It is caused by an expansion of CAG triplets in the HTT gene, and is characterized by progressive decline in motor function and cognition and the development of psychiatric symptoms. The SCA family comprises more than 35 subtypes of progressive neurodegenerative disorders so far, 6 of which are polyQ disorders. The worldwide prevalence of all SCAs combined is around 4/100,000, with geographical variability in some subtypes. SCA3, also known as Machado-Joseph disease (MJD), accounts for 15-45\% of dominantly inherited ataxia in different countries and ethnic populations. Thus, although SCA3 is relatively rare globally, with a prevalence of $1-2 / 100,000$, it is presently considered the most common subtype of SCA worldwide [5,6]. It is caused by a polyQcoding expansion in the ATXN3 gene, and is primarily characterized by neuronal dysfunction and degeneration in the cerebellum and functionally related brain regions. There are no effective treatments for these progressive and fatal disorders yet.

These polyQ disorders share many clinical and pathological features. One classic histopathological hallmark of all polyQ disorders is the insoluble intracellular aggregates or inclusion bodies formed by the various expanded polyQ-encoding proteins in different regions of the neuronal tissue, and the nucleus is thought to be the principal site of the majority polyQ disorder pathogenesis with the exception of SCA2 and SCA6 [7,8]. Intriguingly, the polyQ-containing proteins associated with each different disorder are expressed throughout the body, while the 


\begin{tabular}{|c|c|c|c|c|c|c|c|c|c|}
\hline \multirow{2}{*}{$\begin{array}{l}\text { PolyQ } \\
\text { disorders }\end{array}$} & \multirow[t]{2}{*}{ Prevalence } & \multirow[t]{2}{*}{ Gene } & \multirow{2}{*}{$\begin{array}{l}\text { Chromosome } \\
\text { location }\end{array}$} & \multirow{2}{*}{$\begin{array}{l}\text { Protein } \\
\text { (length, } \\
\text { MW) }\end{array}$} & \multicolumn{2}{|c|}{ PolyQ expansion } & \multirow{2}{*}{$\begin{array}{l}\text { Typical onset } \\
\text { (years) }\end{array}$} & \multirow[t]{2}{*}{ Affected brain areas } & \multirow[t]{2}{*}{ Main clinical symptoms } \\
\hline & & & & & Normal & Pathological & & & \\
\hline HD & $5-10 / 100,000$ & $H T T$ & $4 p 16.3$ & $\begin{array}{l}\text { Huntingtin } \\
\text { (3142 aa, } \\
348 \mathrm{kDa} \text { ) }\end{array}$ & $6-35$ & $36-121$ & $\begin{array}{l}\text { Third to fifth } \\
\text { decades }\end{array}$ & $\begin{array}{l}\text { Striatum, cortex, } \\
\text { thalamus and } \\
\text { subthalamic nucleus }\end{array}$ & $\begin{array}{l}\text { Chorea, dystonia, } \\
\text { hypometric saccades and } \\
\text { catchy pursuit, disrupted } \\
\text { fine motor movements, } \\
\text { dysphagia, dysarthria, } \\
\text { dysdiadokinesis, rigidity, } \\
\text { ataxia, cachexia, } \\
\text { progressive dementia } \\
\text { and psychiatric } \\
\text { symptoms }\end{array}$ \\
\hline SBMA & $1-2 / 100,000$ & $A R$ & $X q 11-q 12$ & $\begin{array}{l}\text { AR (920 aa, } \\
100 \text { kDa) }\end{array}$ & $6-36$ & $38-62$ & $30-60$ & $\begin{array}{l}\text { Lower motor neurons in } \\
\text { the anterior horn, bulbar } \\
\text { region and dorsal root } \\
\text { ganglia }\end{array}$ & $\begin{array}{l}\text { Slowly progressive } \\
\text { muscle weakness and } \\
\text { atrophy of bulbar, facial } \\
\text { and limb muscles }\end{array}$ \\
\hline DRPLA & $<1 / 100,000$ & ATN1 & $12 \mathrm{p} 13.31$ & $\begin{array}{l}\text { Atrophin-1 } \\
(1191 \mathrm{aa}, \\
125 \mathrm{kDa})\end{array}$ & $3-38$ & $49-88$ & $\begin{array}{l}\text { Vary from early } \\
\text { childhood to } \\
\text { late adulthood }\end{array}$ & $\begin{array}{l}\text { Purkinje cells, cerebral } \\
\text { cortex, globus palidus, } \\
\text { striatum, dentate, } \\
\text { subthalamic and red } \\
\text { nuclei }\end{array}$ & $\begin{array}{l}\text { Ataxia, chorea, } \\
\text { myoclonic epilepsy and } \\
\text { dementia }\end{array}$ \\
\hline SCA1 & $1-2 / 100,000$ & $A T X N 1$ & $6 p 23$ & $\begin{array}{l}\text { Ataxin-1 } \\
(815 \mathrm{aa} \\
87 \mathrm{kDa})\end{array}$ & $6-44$ & $49-91$ & $\begin{array}{l}\text { Third or fourth } \\
\text { decade }\end{array}$ & $\begin{array}{l}\text { Cerebellar purkinje cells, } \\
\text { inferior olive neurons } \\
\text { and neurons within } \\
\text { brainstem cranial nerve } \\
\text { nuclei }\end{array}$ & $\begin{array}{l}\text { Ataxia, dysarthria, } \\
\text { ophthalmoparesis, } \\
\text { muscle wasting, } \\
\text { extrapyramidal and } \\
\text { bulbar dysfunction }\end{array}$ \\
\hline SCA2 & $1-2 / 100,000$ & $A T X N 2$ & $12 q 23-24.1$ & $\begin{array}{l}\text { Ataxin-2 } \\
(1313 \mathrm{aa} \\
140 \mathrm{kDa})\end{array}$ & $14-32$ & $33-200$ & Third decade & $\begin{array}{l}\text { Purkinje and granule } \\
\text { neurons }\end{array}$ & $\begin{array}{l}\text { Progressive gait and limb } \\
\text { ataxia, dysarthria, } \\
\text { tremor, nystagmus, slow } \\
\text { saccadic eye movement } \\
\text { and supranuclear } \\
\text { ophtalmoplegia }\end{array}$ \\
\hline SCA3 & $1-2 / 100,000$ & ATXN3 & $14 q 32.1$ & $\begin{array}{l}\text { Ataxin-3 } \\
(364 \mathrm{aa}, \\
42 \mathrm{kDa})\end{array}$ & $12-44$ & $50-89$ & $20-50$ & $\begin{array}{l}\text { Cerebellum and } \\
\text { brainstem, basal ganglia, } \\
\text { thalamus, substantia } \\
\text { nigra and spinal cord }\end{array}$ & $\begin{array}{l}\text { Cerebellar ataxia, } \\
\text { progressive external } \\
\text { ophthalmoplegia, } \\
\text { dysarthria, dysphagia, } \\
\text { pyramidal signs, } \\
\text { dystonia, rigidity and } \\
\text { distal muscle atrophies, } \\
\text { weight loss and restless } \\
\text { legs syndrome }\end{array}$ \\
\hline SCA6 & $<1 / 100,000$ & CACNA1A & $19 \mathrm{p} 13$ & $\begin{array}{l}\text { CACNA1A } \\
(2505 \mathrm{aa} \\
282 \mathrm{kDa})\end{array}$ & $3-18$ & $21-33$ & $19-71$ & Cerebellar purkinje cells & $\begin{array}{l}\text { Gait unsteadiness, } \\
\text { stumbling and imbalance }\end{array}$ \\
\hline SCA7 & $<1 / 100,000$ & ATXN7 & $3 p 21-p 12$ & $\begin{array}{l}\text { Ataxin-7 } \\
(892 \mathrm{aa} \\
95 \mathrm{kDa})\end{array}$ & $4-35$ & $36-460$ & $\begin{array}{l}\text { Third or fourth } \\
\text { decade }\end{array}$ & $\begin{array}{l}\text { Cerebellar cortex, deep } \\
\text { cerebellar nuclei, inferior } \\
\text { olive and anterior horns } \\
\text { of the spinal cord, as } \\
\text { well as axonal loss in } \\
\text { spinocerebellar tracts }\end{array}$ & $\begin{array}{l}\text { Cerebellar ataxia, } \\
\text { uncoordinated } \\
\text { movement, abnormal } \\
\text { gait, dysarthria and } \\
\text { dysphagia, degeneration } \\
\text { of the retinal macula }\end{array}$ \\
\hline SCA17 & unknown & $T B P$ & $6 q 27$ & $\begin{array}{l}\text { TBP (338 } \\
\mathrm{aa}, 38 \mathrm{kDa})\end{array}$ & $25-44$ & $45-63$ & Middle age & $\begin{array}{l}\text { Small neurons in the } \\
\text { caudate and putamen, } \\
\text { purkinje cells and frontal } \\
\text { and temporal cortex }\end{array}$ & $\begin{array}{l}\text { Ataxia, dystonia and } \\
\text { parkinsonism, dementia, } \\
\text { psychiatric abnormalities } \\
\text { and seizures }\end{array}$ \\
\hline
\end{tabular}

pathology is primarily restricted to neuronal tissue. These disorders further share a negative correlation between the polyQ expansion length and the age of disease onset $[7,9,10]$. Transcriptional dysregulation and defects in the ubiquitin proteosome system are other important common aspects to all of these disorders [11]. Moreover, many of the polyQ-encoding proteins have been shown to share interacting partners. For example, both Huntingtin (Htt) and ataxin-3 interact with p53, CBP and p300 [12-14]. Currently, the precise pathogenic mechanism in polyQ disorder patients remains elusive $[1,2,12,15-17]$. 
The shared features of these disorders indicate similarities in disease mechanisms relating to the expanded polyQ. As no cases of polyQ diseases with deletions or point mutations in their causative genes have been reported, the polyQ expansion is thought to confer a toxic gain-of-function to the affected proteins and trigger a pathogenic cascade, leading to distinct patterns of neuronal loss and clinical manifestation. The involvement of the polyQ expansion in the mechanism leading to the disease includes induction of conformational transition of the host protein, alteration of the normal protein function, generation of toxic polyQ-containing fragments, transcriptional disturbances, proteotoxic stress and mitochondrial dysfunction [3,18]. However, it is noteworthy that the polyQ tract within a protein is not always deleterious. For example, aggresomes formed by expanded polyQ protein, which is different from the small aggregates or oligomers formed by the self-association of the proteins, protect cells by enhancing the degradation of toxic polyQ-containing proteins [19,20]. The aggregates or intranuclear inclusions formed by misfolded and expanded disease proteins have been reported to be dissociated from the pathogenic process in HD and SCA1 [3,21-23].

\section{Epigenetic mechanisms}

Epigenetics is commonly defined as stable and heritable changes in gene expression or genome function without changes in the genotype. Modification of epigenetic processes, which can be influenced by a number of internal and external environmental risk factors, may alter chromosomal stability and gene expression and affect the phenotype of an organism. The role of epigenetics in human diseases is first recognized in oncology, but in the last decade the contribution of epigenetic modifications to neurodegenerative disorders, such as Alzheimer's disease (AD), Parkinson's disease (PD) and HD, has also been extensively investigated. Primary epigenetic mechanisms include DNA methylation and hydroxymethylation, histone modifications and chromatin remodeling and more recently noncoding RNA regulation [24].

DNA methylation \& hydroxymethylation

DNA methylation is one of the most studied and best characterized epigenetic modifications. DNA methylation in mammals, catalyzed by DNA methyltransferases (DNMTs) using 5-adenosylmethionine (SAM) as the methyl donor [25], mainly occurs on the cytosine nucleotide in a CpG site, forming 5'-methylcytosine (5-mC) [26,27]. There are also reports of $\mathrm{CpH}$ methylation as well as guanine and adenine methylation [28,29]. How DNA methylation affects gene transcription is highly dependent on the location in or around the gene [30]. DNA methylation in promoter regions generally acts to repress gene transcription [25], while DNA methylation within gene bodies is reported to be associated with splicing-related regulation at introns [31,32]. 5-hydroxymethylcytosine (5-hmC) [3337] and 5-formylmethylcytosine, two intermediate states of the DNA demethylation, are thought to act as newly functional epigenetic markers. They are present in high prevalence in the brain $(\sim 8 \%$ for 5 -hmC and $\sim 0.8 \%$ for 5-formylmethylcytosine, in contrast to $\sim 80 \%$ for 5 -mC), associating with relief of transcriptional silencing $[38,39]$.

\section{Histone modifications \& chromatin remodeling}

Gene expression is generally regulated by the binding of transcriptional coregulators and the chromatin structure alterations, with the latter being primarily regulated by histone modifications. Nucleosome, the fundamental unit of chromatin, is a histone octamer consisting of two molecules of each core histone $(\mathrm{H}) \mathrm{H} 2 \mathrm{~A}, \mathrm{H} 2 \mathrm{~B}, \mathrm{H} 3$ and $\mathrm{H} 4$ around which $147 \mathrm{bp}$ of DNA is wrapped. The histone tail, which is the flexible N-terminals of histones protruding from the nucleosome, is subjected to numerous post-translational modifications on multiple residues (known as the so called 'histone code'), such as acetylation, methylation, phosphorylation, ubiquitination, sumoylation, ADP-ribosylation, carbonylation, glycosylation, biotinylation and hydroxylation, among which lysine acetylation and lysine methylations are particularly important [40]. The histone code plays important roles in gene expression regulation by altering chromatin dynamics and influencing histone-DNA interactions, as well as recruiting regulatory proteins and enzymes to chromatin.

Acetylation of histones, catalyzed by histone acetyltransferases (HATs), is generally believed to lead to the decondensation of the chromatin and promote transcriptional activity, whereas histone deacetylases (HDACs) exert the opposite effect [41]. In addition to remodeling chromatin, acetylated-lysine residues may act as transcriptional activators, thus indirectly accelerating transcriptional initiation.

In contrast to histone acetylation, histone methylation, which is regulated by the counteracting activity of histonemethyltransferases and histone-demethylases, appears to be more specific. Lysine residues of histone proteins are able to be mono-, di- or tri-methylated by specific enzymes while arginine is able to be mono- or di-methylated. 
Histone methylation can either increase or decrease the gene expression, depending on which amino acids are methylated and the number of methyl groups that are added to these residues. For example, hypermethylation of histone $\mathrm{H} 3 \mathrm{~K} 9$ and $\mathrm{H} 4 \mathrm{~K} 20$ residues frequently represses transcription while di- and tri-methylation of $\mathrm{H} 3 \mathrm{~K} 4$, $\mathrm{H} 3 \mathrm{~K} 36$ and $\mathrm{H} 3 \mathrm{~K} 79$ are generally correlated with active chromatin [42]. The interplay between different histone modifiers determines chromatin structure and function. In addition, chromatin remodelers, including SWI/SNF, ISWI, CHD and INO80 family, can regulate the chromatin accessibility by modifying the presence, composition and nucleosome positioning [43].

\section{Noncoding RNAs}

The best characterized group of ncRNAs is miRNAs. miRNAs are small, 18-22 nt long ncRNAs that represent a major system of post-transcriptional regulation. They have been shown to regulate gene expression by promoting either degradation or translational repression of target mRNAs [44]. The expression of miRNAs varies with developmental processes, suggesting their involvement in various developmental processes such as cell fate determination, cell division and programmed cell death. In mammals, the majority of miRNAs are expressed abundantly in the CNS in specific spatial and/or temporal patterns, suggesting their potential roles in neurodevelopment, nervous system morphogenesis, synaptic plasticity and neurodegeneration.

\section{Epigenetic crosstalk}

Proteins that regulate DNA methylation are associated with proteins that regulate histone modifications, thus linking these two epigenetic processes. For example, MeCP2 is associated with HDACs, thus linking DNA methylation and histone deacetylation [45]. Generally, DNA methylation could exert great impact on interaction between histone and DNA, changing chromosome structure and gene expression [46]. In addition, DNA methylation and histone modification can regulate miRNA expression and vice versa. For example, treatment by DNA demethylation agent and/or HDAC inhibitors causes miRNA expression changes in cells [47,48], and enzymes involved in DNA methylation and histone modification, such as members of DNMTs [49,50] and DNA methyl-CpG-binding proteins (MBDs) as well as HDACs, are targeted by miRNAs [51,52]. For a more comprehensive view of different epigenetic marks crosstalk see [53].

\section{Epigenetic alterations in polyQ disorders}

$\mathrm{HD}$, as well as other polyQ diseases, is a single gene disorder. However, there is enormous variability in disease at onset and severity, suggesting that other genetic and/or environmental factors may influence disease phenotypes. Accumulating evidences show that the alteration of epigenetic processes, such as DNA methylation and posttranslational modifications of histone proteins, is linked pathologically with polyQ disorders in many aspects, such as modification of the disease progression and regulation of the instability of CAG repeats expansion, thus providing an epigenetic mechanism of gene regulation in these disorders. In this review, we first focus on the roles of DNA methylation and histone modifications in the pathological process of several polyQ disorders. We then summarize recent findings that highlight the association of miRNAs with polyQ disorders and discuss the potential therapeutic applications of miRNAs as targets to treat polyQ disorders, as well as their utilization as biomarkers.

Methylation-based epigenetic regulation in polyQ disorders

Alteration of methylation in $H D$

Mounting evidence in the literature supports the notion that aberrant DNA methylation is potentially linked with HD-related transcriptional dysregulation and neuronal dysfunction, as summarized below [54].

Global DNA (de)methylation changes in HD

Global levels of DNA methylation have been studied in cell lines and different mouse models of HD as well as HD patient samples. An immortalized mouse striatal cell line carrying polyQ-expanded HTT (STHdhQ111/Q111) shows a general tendency toward hypomethylation as measured by reduced representation bisulfite sequencing [55]. Similar results have been obtained in fibroblasts from HD patients [56. In human HD brain and animal models, global levels of 7-methylguanosine are significantly reduced in the motor cortex [28]. A previous study from our lab reports the genome-wide DNA hydroxymethylation in HD mouse brain, with global reduced 5-hmC being observed in the striatum and cortex of YAC128 transgenic mice [57]. These studies indicate that HD is generally associated with lower levels of DNA methylation independently of the sample used. Dysregulation of 
other methylated marks, such as 7-methylguanosine and 5-hmC, may also contribute to the known transcriptional pathology of HD.

\section{Gene-specific DNA methylation changes in HD}

Gene-specific DNA methylation changes have focused on genes related to HD [56,58]. For example, adenosine $\mathrm{A} 2 \mathrm{~A}$ receptor $(\mathrm{A} 2 \mathrm{AR})$ gene $(A D O R A 2 A)$ is a gene that shows severely decreased expression in HD patients (7). An increase in 5-mC levels and a reduction in 5-hmC levels in the striatal 5'UTR of $A D O R A 2 A$ of HD patients have been observed, with both mechanisms being related to the pathological decrease of $A D O R A 2 A$ in HD [58]. However, this differential methylation of the $A D O R A 2 A$ gene may be driven by different cell-type proportions in brain instead of disease status [59]. Another example is hairy and enhancer of split 4 (HES4), a notch signaling gene which is recently suggested to be linked with HD pathogenesis. Hypermethylation of the HES4 promoter has been observed in HD cortical neurons [60]. Therefore, these studies show that some HD-associated genes are epigenetically dysregulated at the DNA methylation level in HD, but they require further validations using cell-type-specific studies.

\section{DNA methylation changes at the HTT locus}

There are also studies to investigate how the HD mutation impact local DNA methylation patterns at the HTT locus itself $[59,61,62]$. A more than 28-fold of variability of methylation in DNA from peripheral blood has been detected at a locus (D4S95) closely linked to the HTT gene [61]. No HD-associated DNA methylation changes at the HTT locus has been detected in HD cortex samples [59], and 38 sites within the HTT gene locus are differentially methylated between matched cortex and liver samples [59]. Therefore, no conclusive evidences have emerged from these studies. However, it should be noted that due to technique limitations, these studies fail to assess DNA methylation levels within the CAG tract of mutant HTT loci directly.

\section{Alteration of DNA methylation-related gene expression in HD}

Recent studies have also explored the possible causes for DNA methylation difference observed in HD. As mentioned above, DNA methylation in mammals is mainly catalyzed by DNMTs. Decreased expression of DNMT genes has been found in HD models by independent groups [55,63-65]. Moreover, several other DNA (de)methylation-related genes, such as Gadd45a [56,65], Gadd45g [55] and Rnf4 [56,63], have also been found to be differentially expressed in HD models. These studies would partly explain the correspondingly lowered levels of DNA methylation in HD.

Alteration of methylation in SCAs

Two large CpG islands are found in the promoter region (from -1089 to +1 ) of the ATXN3 gene [66], with the methylation levels in the first $\mathrm{CpG}$ island being significantly increased in SCA3/MJD patients and the second CpG island being hypomethylated in both patients and controls, suggesting an essential role of the first but not the second island DNA methylation in the SCA3 pathogenesis [67]. Genome-wide methylation analysis shows that ATXN2 is significantly methylated in a case series of coronary artery disease [68]. The ATXN2 promoter contains a CpG island rich segment without a TATA box. Both hyper- and hypomethylation states are found in the ATXN2 promoter in SCA2 patients [69,70]. In SCA1, local DNA methylation patterns have been detected at ATXN1 locus. Dnmt1 deficiency leads to opposite effects on local Scal DNA methylation in testes and ovaries of Dmnt1+/-SCA1 mice, with elevated levels in testes and reduced levels in ovaries using a mouse model for SCA1 [71]. In the case of SCA7, it is reported that CpG methylation of CTCF-binding site adjacent to the expanded CAG tracts of ATXN7 could enhance triplet repeat instability [72].

Taken together with data from these polyQ disease models, although the changes of DNA methylation or hydroxymethylation are relatively small in these studies, and the interpretation of these results can be influenced by many factors, such as the sample size, tissue difference, data analysis method and different measure techniques, they illustrate that aberrant DNA methylation is a key feature in the transcriptional dysregulation observed in polyQ diseases, which may, at least in part, contribute to altered gene expression and neuronal dysfunction, thus playing an important role in the transcriptional pathology of these diseases. Future studies aiming at assessing specific forms of DNA methylation using cell-type-specific studies will advance our understanding of the role of DNA methylation in the pathogenesis of these diseases. 
Histone modifications \& chromatin remodeling in polyQ disorders

Histone modifications, such as hypo/hyperacetylation and hypermethylation, have been identified in cell models of HD, HD animal models and patients [64,73-76]. Global levels of histone acetylation are reduced in HD models [76,77] and in HD patients [78-81], although hyperacetylation at certain gene loci is shown to be increased [82,83]. Different truncated Htt with an expanded polyQ domain has been found to inhibit the HAT function and reduce histone acetylation in cell culture by independent groups [76,84]. Similarly, in a transgenic SCA3 mouse model, mutant ataxin-3 impairs HAT activity, leading to hypoacetylation of $\mathrm{H} 3$ or $\mathrm{H} 4$ histone and further the transcriptional repression of cerebellar genes required for long-term depression (LTD) [85,86]. A reduction in acetylation induced by expanded polyQ proteins is also observed in yeast and in a cell model of Kennedy's disease [76. Therefore, these studies strongly implicate that histone hypoacetylation caused by a reduced HAT activity may be an important component of polyQ pathogenesis, and HDAC inhibitors may represent a relevant therapeutic strategy for polyQ diseases.

The wild-type Htt protein interacts with various histone modifiers such as CBP, TBP, p300 and REST/NRSF [87,88]. The abnormal histone acetylation in HD is related to the loss of CBP, a transcriptional cofactor with HAT activity. The stronger binding of CBP with mHtt leads to its sequestration in mHtt-induced inclusions and to the hypermethylation and hypoacetylation of histones, and the subsequent neuronal transcriptional dysfunction in brains of HD mice [74,77,82,89-92]. Moreover, a loss of CBP function results in an increase in H3K9me3, which is linked to upstream transcriptional dysregulation [79,89,93]. In agreement, upregulation of CBP rescues HD phenotypes in a Drosophila model of HD, which is associated with recovery of histone acetylation and normalization of the transcription profile [94]. However, partial deletion of CBP in HD transgenic mice fails to affect the global levels of histone $\mathrm{H} 3$ or $\mathrm{H} 4$ acetylation in the brain [95]. Further investigation is required to study the role of CBP in HD.

Additionally, mHtt can alter histone methylation [89,90,93] and ubiquitination [96-98]. For example, increased levels of ubiquitinated $\mathrm{H} 2 \mathrm{~A}$ has been observed in a cell model of $\mathrm{HD}$ and in $\mathrm{HD}$ mice, which is due to a disruption of the interaction between $\mathrm{mHtt}$ and Bmi1, a component of the hPRC1L E3 ubiquitin ligase complex [97].

Similar to Htt, some polyQ-encoding proteins in SCAs can also interact with various histone modifiers, thus linking them directly with chromatin regulation. For example, ataxin-1 interacts with Tip60, which is involved in transcriptional activation by acetylating $\mathrm{H} 4$ and $\mathrm{H} 2 \mathrm{~A}$ [99], whereas ataxin-3 can interact with CBP, p300, HDAC6 and PCAF [100-102]. SCA7 is caused by polyQ expansion of the ataxin-7, which is a component of the mammalian SAGA and SLIK HAT complexes [103]. Mutation of ataxin-7 leads to the disruption of the structural integrity of the SAGA complex and aberrant chromatin acetylation patterns at the promoters of genes [104].

Taken together, these studies implicate the important roles of histone modification and chromatin remodeling in the pathogenesis of polyQ diseases as well as in the regulation of the polyQ-encoding protein function, and chromatin-directed compounds such as HDAC inhibitors could be developed as potential drugs for polyQ diseases in the future.

\section{Dysregulation of miRNA in the pathophysiology of polyQ disorders}

miRNA dysregulation is emerging as a critical factor contributing to neurodegeneration [105-112]. Major alterations in brain mRNA levels have been observed in various neurodegenerative disorders, and as mentioned above, altered neuronal transcriptional activity is a persistent feature of most polyQ disorders. More and more miRNAs have been identified to play potential roles in polyQ disorders, either by post-transcriptionally regulating the expression of polyQ disease causing proteins in mammals or by modulating toxicity through miRNA-mediated mechanisms. Bilen et al. provided the first evidence that the miRNA pathways play protective roles in the polyQ neurodegeneration in both flies and human cells [113,114]. They have identified the anti-apoptotic miRNA bantam (ban) to prevent degeneration caused by the mutant ataxin-3 in Drosophila by modulating polyQ toxicity [113,114]. This finding has been supported by other studies $[115,116]$. For example, Liu et al. report that upregulation of a markedly conserved miRNA, miR-34, dramatically mitigate neurodegeneration induced by polyQ expanded mutant ataxin-3 in flies, thus supporting the protective role of miRNAs in SCA3 [117]. In the section below, we will discuss several brain-enriched miRNAs as well as circulating miRNAs (cmiRNAs) implicated in HD and other polyQ disorders. 


\section{Brain-enriched miRNAs dysregulation in poly $Q$ disorders}

Many transcription factors have been reported to interact with $\mathrm{Htt}$ and are recruited to the mHtt aggregates in the brain. Importantly, mHtt can interact with Ago1 and Ago2, which are involved in miRNA biogenesis, resulting in the inhibition of the formation of processing bodies [118]. Therefore, miRNA dysregulation is expected in HD. Widespread neuronal-specific miRNA dysregulation has been detected in the brains of HD animal models and HD patients [119-121], with some miRNAs being downregulated, such as miR-29b, miR-125b, miR-146a and miR-150 [122-124], and some miRNAs being significantly upregulated, such as miR-10b-5p, miR-196a-5p, miR-196b-5p, miR-200a and miR-200c (Table 2) [124-127]. However, it should be noted that these studies show considerable heterogeneity between different species of disease samples, and only a small fraction of miRNAs have been confirmed by different groups. Some of the studies appear compelling. For example, miR-132, a brain-enriched miRNA, is first found significantly depressed in both human and mouse HD samples [128,129], which has been confirmed later by Lee et al. [130] in two different HD mouse models. However, Packer et al. observe no change in miR-132 at stages 1 and 2, but significant upregulation later in human brain samples of HD grades 1-4 [124]. These seemingly contradictory results are probably because different miRNAs (precursor vs mature miRNA) are tested.

miRNA dysregulation in HD has been reported to be partly due to aberrant increased expression and the nuclear localization of REST [129]. REST and CoREST, two components of the REST complex, are known to suppress the expression of neuronal genes in non-neuronal cells. In healthy neurons, REST is primarily sequestered in the cytoplasm partly through binding to Htt [131]. However, in HD patients, the polyQ expansion abrogates REST-Htt binding, enabling its nuclear translocation, occupies RE1 repressor sequences and decreases neuronal gene expression such as BDNF [132,133]. Therefore, REST is thought to be one of the downstream effectors of HTT in HD pathology. A set of REST-target miRNAs including miR-29a, miR-124a, miR-132 and miR-330 have been found to be decreased in a mouse model of HD, among which only miR-132 downregulation has been confirmed in human samples [129].

Similar to the case in HD, alteration of numerous mRNA transcripts as well as several miRNA levels have been observed in brains of SCA sufferers as well as animal models [134,135]. Interestingly, ataxin-2, the protein involved in SCA2 disease, might be required for miRNA function, as lack of ataxin-2 impairs the repressive activity of several miRNAs [136]. In SCA3, miR-181a and miR-494, which interact with the ATXN3-3'-UTR, are found dysregulated in human MJD neurons as well as other MJD cell and animal models, and overexpression of these miRNAs alleviated MJD neuropathology in vivo [137]. In SCA17, downregulation of miR-29a and miR-29b is observed in a cellular model of SCA17, which was inversely correlated with BACE1 expression [138]. In addition, TBP gene was reported to be one of the targets of miR-146a, which was downregulated in cell and animal models of HD [122]. Studies of the dysregulation of miRNA expression in different SCA1 animal models as well as SCA1 patients show that most of miRNAs were downregulated, and only a small fraction of miRNAs were upregulated, such as miR-19a, miR-101, miR-130a, miR-144 and miR-150 [139-141].

It is important to mention that some miRNAs have been found to be involved in more than one neurodegenerative disease conditions. Here, we take miR-9 for example. miR-9 is one of the most abundant miRNAs in the brain, which has RE1 binding sites, and is a direct target of REST. Levels of mature miR-9/miR-9* are decreased in cell models of HD and HD mouse models as well as HD patients [122,124,128]. Decreased miR-9/9* in HD would increase REST transcription, amplifying its accumulation in the presence of $\mathrm{mHtt}$. This phenomenon is further magnified because miR-9/9* transcription depends on REST. Aberrant expression of miR-9 has also been observed in the cerebella of mouse model of SCA3 [137] and SCA1 [140], being downregulated in SCA3 and upregulated in SCA1. The miR-9 dysregulation in SCA3 is proposed to be related with an impairment in miRNA biogenesis. In contrast, both upregulation $[142,143]$ and downregulation $[144,145]$ of miR-9 have also been reported in AD models. These dysregulations are thought to be associated with one of its target BACE1 as well as A $\beta$ accumulation [146].

Another miRNA, miR-29a, has also been found to be dysregulated in three polyQ disorders, including HD [129], SCA3 [147] and SCA17 [138], due in large part, to the disrupted miRNA transcriptome. miR-29a has also been found to be decreased in $\mathrm{AD}$ patients and $\mathrm{AD}$ mice models [144,145], which is correlated with the increase of BACE1, a confirmed target of miR-29.

Taken together, the involvement of miRNA dysregulation in different neurodegenerative diseases, either causally or as part of positive feedback loops, suggests that each miRNA can regulate numerous targets and play different roles in the brain with different mechanisms. 
Table 2. Aberrant expression of miRNAs in polyglutamine disorders.

PolyQ miRNAs

Changes disorders

HD

miR-9/9*, miR-124, miR-132

Downregulated

Sources

Species

Ref.

miR-29a, miR-124a, miR-132, miR-135b, miR-204

Downregulated

Cortex

Human and

[128]

(n)

miR-132

miR-29a, miR-330

Downregulate

Cortex and/or

mice

hippocampus

Mice

miR-9/9*, mir-29b, miR-124a, miR-132

Upregulated

miR-128

Downregulated

Cortices

Human

miR-22, miR-29c, miR-128, miR-132, miR-138, miR-218, miR-222,

Upregulated

miR-344, miR-674*, miR-28*, miR-466h

miR-34b-3p, miR-207, miR-448, miR-669c, miR-18a*

miR-183, miR-96

miR-200a, miR-200b, miR-429

Downregulated

Cortex (Brodmann's Human

area 4)

miR-9/9*, miR-100, miR-125b, miR-135a, miR-135b, miR-138

miR-146a, miR-150, miR-181c, miR-190, miR-218, miR-221, miR-222, miR-338-3p

miR-145, miR-199-5p, miR-199-3p, miR-148a, miR-127-3p, miR-200a, Upregulated miR-205, miR-214, miR-335-5p, miR-299-5p, miR-323-3p, miR-154

miR-185, miR-194, miR-128a, miR-33a, miR-320, miR-17-3p,

Downregulated

Frontal cortex

Monkey

miR-181c, miR-220b, miR-940

miR-451, miR-133c

Upregulated

miR-124, miR-127-3p, miR-128, miR-139-3p, miR-181d, miR-221, Downregulate

Frontal cortex and Human

the striatum

miR-485-3p, miR-485-5p, miR-95

miR-100, miR-106b, miR-148b, miR-151-3p, miR-151-5p, miR-15b, Upregulated

miR-16, miR-17, miR-193b, miR-19b, miR-20a, miR-219-2-3p,

miR-219-5p, miR-27b, miR-33b, miR-363, miR-451, miR-486-5p,

miR-887, miR-92a

miR-432, miR-146a, miR-19a

Downregulated

STHdh $h^{Q 111} / H d h^{Q 111}$ Mice

cells

miR-10b-5p, miR-196a-5p, miR-196b-5p, miR-615-3p, miR-10b-3p, miR-1247-5p

miR-674-5p, miR-221, miR-24*, miR-693, miR-674-3p, miR-34a miR-711, miR-143, miR-138, miR-222, miR-326, miR-216a, miR-221, miR-448, miR-199b

miR-141, miR-182, miR-429, miR-200c, miR-183, miR-200a, miR-96, Upregulated

miR-152, miR-190, miR-496, miR-181d, miR-805, miR-369-3p,

miR-384, miR-361, miR-135a, miR-136, miR335, miR-706, miR-365, miR-744, miR-703, miR-694, miR-761

miR-10b-5p, miR-486-5p

Upregulated

Plasma

Human

miR-34b

miR-877-5p, miR-223-3p, miR-223-5p, miR-30d-5p, miR-128, Upregulated

Plasma

Human

miR-22-5p, miR-222-3p, miR-338-3p, miR-130b-3p, miR-425-5p

Upregulated

Plasma

Human

[151]

miR-628-3p, miR-361-5p, miR-942

SCA3

miR-25, miR-125b, miR-29a

Downregulate

Serum

Human

$[147,167]$

miR-34b

miR-33-5p, miR-92a, miR-100-5p

Upregulated

Upregulated

Head

Drosophila

[168]

miR-1-3p

miR-9, miR-181a, miR-494

Downregulated

miR-144, miR-101, miR-130a, miR-19a, miR-302

Downregulated

Cerebella

Mice

SCA1

miR-381, miR-203, miR-34c, miR-489, miR-224, miR-484, miR-329, miR-133b, miR-423, miR-138, miR-487b, miR-206

miR-22, miR-125b, miR-194, miR-24, miR-30c, miR-16, miR-191,

miR-143, miR-376b, miR-376a, miR-26a, miR-218, miR-195, miR-361,

Upregulated

Cortex

Human

Downregulated

Cerebella

Mice

miR-150, miR-100, miR-7, miR-146b, miR-335, miR-26b, miR-96,

miR-379, miR-9*, miR-30b, miR-126-3p, miR-128b, miR-9, miR-31,

miR-30d, miR-23a, miR-27a, miR-350, miR-129-3p, miR-99a

HD: Huntington's disease; PolyQ: Polyglutamine; SCA: Spinocerebellar ataxia. 
Table 2. Aberrant expression of miRNAs in polyglutamine disorders (cont.).

\begin{tabular}{|lllll}
\hline $\begin{array}{l}\text { PolyQ } \\
\text { disorders }\end{array}$ & miRNAs & Changes & Sources & Ref. \\
\hline SCA7 & miR-33-5p, miR-92a-5p, miR-34-5p & Upregulated & Head & Drosophila \\
\hline & miR-375-3p & Downregulated & Head & Drosophila \\
\hline SCA17 & miR-33-5p, miR-92a-5p & Upregulated & & \\
\hline HD: Huntington's disease; PolyQ: Polyglutamine; SCA: Spinocerebellar ataxia. & Downregulated & Cell model \\
\hline
\end{tabular}

cmiRNAs dysregulation in polyQ disorders

Outside of the CNS, significant alterations in miRNA expression have also been detected in extracellular fluids in HD and other polyQ disorders [148-151]. For example, miR-10b-5p [148,150] and miR-34b [149] were shown to be significantly elevated in the plasma of HD patients and HD asymptomatic gene carriers, respectively. Most recently, cmiRNAs analysis of plasma samples from $15 \mathrm{HD}$ symptomatic patients shows that $168 \mathrm{cmiRNAs}$ are altered. Most of upregulated miRNAs in HD patients are involved in metabolism regulation [151]. In SCA3, four miRNAs, including miR-25, miR-125b, miR-29a and miR-34b, are dysregulated in the serums of SCA3/MJD patients, with the first three being decreased and the last being dramatically elevated [147]. These results indicate that polyQ patients show markedly altered cmiRNA expression pattern, although the levels of some of the dysregulated miRNAs may be different in brain and plasma. These findings implicate that serum miRNAs, due to their stability and specificity, have the potential to be useful biomarkers for these disorders, thus being clinically beneficial for the diagnosis of polyQ patients.

Taken together, these studies provide evidences for a direct or indirect role of miRNAs in the pathogenesis of polyQ disorders and open the possibility for miRNA-based diagnosis, prognosis and therapeutic developments. However, it is quite possible that not all the identified miRNAs are directly associated with neurodegeneration in polyQ diseases although they are dysregulated. The precise and detailed roles of individual miRNAs demand further studies in future.

\section{Potential therapeutic roles of miRNAs to treat polyQ disorders}

The gene regulation role of miRNAs suggests a potential therapeutic approach of post-transcriptional silencing that targets the underlying disease etiology rather than the downstream pathological consequences. Besides, as mentioned above, certain miRNAs target multiple genes related to the diseases in a pathway simultaneously, making them more effective mediators. Therefore, those miRNAs whose expression are dysregulated and are directly associated with neurodegeneration can be biomarkers for the diagnosis and prognostication of these diseases. cmiRNAs, due to their high stability and specificity, may potentially serve as novel noninvasive biomarkers for neurological diseases. Moreover, some miRNAs are common in more than one polyQ disease conditions, making them global targets for novel therapeutic strategies.

Two main miRNA-based therapeutic strategies have been developed in vivo, which are replacing these miRNAs with their synthetic mimics and blocking the miRNAs of interest by anti-miRNA molecules. Modified oligonucleotides as well as peptide nucleic acids oligonucleotides and morpholinos (mainly used in zebrafish system) have been shown to be effective inhibitors of miRNA activity (for reviews, see [152]). In recent years, synthetic sponge miRNAs, which have complementary binding sites to a miRNA of interest, have been developed to inactivate the target miRNAs [153].

Most cases of $\mathrm{AD}$ and $\mathrm{PD}$, the most prevalent neurodegenerative diseases worldwide, are sporadic, meaning no significant associations with certain genes are detected in these cases. However, progress has been made in RNA-based silencing of targets linked to common sporadic forms of AD and PD despite of the difficulty of defining the appropriate therapeutic targets. For instance, the $\alpha$-synuclein pathway has been successfully targeted in $\mathrm{PD}$ [154]. In contrast to $\mathrm{AD}$ and $\mathrm{PD}$, each of the polyQ disorder is caused by a defined genetic mutation. Therefore, it is therapeutically possible that reducing the mutant gene expression may slow or even prevent disease progression. Most of the progress in the development of RNA-based therapies has been made in these hereditary neurodegenerative diseases. We specifically highlight the implications of miRNA mimics in polyQ disorders in this review. 
miRNA mimics have been applied to silence the corresponding mutant genes in different polyQ disorder models, either non-allele specifically or allele specifically [40,155-158]. For example, viral-mediated expression of mi2.4, an artificial miRNA silencing of both mutant and wild-type HTT mRNAs, has been shown to improve HD-related behavioral abnormalities dramatically in HD-N171-82Q mice [157,158]. miR-196a is previously identified as a possible candidate for involvement in the pathogenesis of HD. A double transgenic mouse carrying mutant HTT and miR-196a reveals the suppression of mutant HTT in the brain and also shows improvements in neuropathological progression [159]. Reducing expression of mutant ataxin-1 using virally delivered artificial miRNAs improves disease phenotypes in SCA1 transgenic mice [160] and knock-in mice [161]. Artificial anti-ATXN3 miRNA mimics based on the human miRNA-124 primary sequence have been shown to reduce mutant $A T X N 3$ expression, enhance the clearance of mutant ataxin-3 and partially restore specific miRNA steady-state levels in SCA3/MJD84.2 mice [162]. However, long-term expression of this miRNA mimic fails to alter the progression of motor deficits significantly in homozygote SCA3/84.2 mice [163]. Although miRNA-based therapeutic applications for polyQ disorders are promising, several hurdles should be overcome in the future, such as the mutant allele selectivity, stability, target specificity, efficacy and safety, as well as optimization of the synthetic and delivery techniques. Among these challenges, off-target effects of delivering miRNA targets are particularly important, as they may cause severe unanticipated responses. This is because, as summarized in this review, some miRNAs, such as miR-9 and miR-29a, are involved in more than one polyQ disease conditions. Moreover, these miRNAs have many different downstream targets, but only minor of them have been identified.

\section{Future perspective}

The importance of epigenetic variation in the neuronal development and activity is increasingly recognized [164]. A growing body of evidence reveals that epigenetic modifications contribute to the pathology of different kinds of polyQ disorders. However, to date, the extent to which epigenetic modification may modulate the onset, progression or severity of a specific polyQ disorder remains largely unknown. Here, we summarize epigenetic changes in several representative polyQ disorders. There remain a number of future challenges that need to be addressed before we can fully understand the mechanisms and consequences of the epigenetic regulation in the neuronal development and activity. There is a need to identify the specific DNA methylation maintenance factors that are involved in each polyQ disorder in the future. How miRNAs exactly mediate the pathological mechanisms in polyQ disorders also await further investigation. In addition, it is crucial to understand to what extent these epigenetic variation contribute to the pathogenesis of polyQ disorders.

Executive summary

Epigenetic alterations in polyglutamine disorders

- Aberrant DNA methylation is a key feature in the transcriptional dysregulation observed in polyglutamine (polyQ) diseases.

- Histone modification and chromatin remodeling are involved in the pathogenesis of polyQ diseases as well as in the regulation of the polyQ-encoding protein function, and chromatin-directed compounds such as histone deacetylase inhibitors could be developed as potential drugs for polyQ diseases in the future.

- Dysregulation of miRNAs is involved in different neurodegenerative disease processes, either causally or as part of positive feedback loops.

Potential therapeutic roles of miRNAs to treat polyQ disorders

- Diagnosis and prognosis: circulating miRNAs, whose expression are dysregulated and are directly associated with neurodegeneration, may potentially serve as novel noninvasive biomarkers for polyQ diseases.

- Treatment: replacing miRNAs with their synthetic mimics as a miRNA-based therapeutic application for polyQ disorders is promising.

Future perspective

- There is a need to identify the specific DNA methylation maintenance factors that are involved in each polyQ disorder in the future.

- How miRNAs exactly mediate the pathological mechanisms in polyQ disorders await further investigation.

- It is crucial to understand to what extent these epigenetic variation contribute to the pathogenesis of polyQ disorders. 
Financial \& competing interests disclosure

This work was supported by NSFC (91754204, 31300880), National Key Research and Development Program of China 2017YFC1001001, NSFC81630078, CAS Strategic Priority Research Program XDB14030300, and the State Key Laboratory of Membrane Biology. The authors have no other relevant affiliations or financial involvement with any organization or entity with a financial interest in or financial conflict with the subject matter or materials discussed in the manuscript apart from those disclosed.

No writing assistance was utilized in the production of this manuscript.

\section{References}

Papers of special note have been highlighted as: $\bullet$ of interest; $\bullet \bullet$ of considerable interest

1 Gatchel JR, Zoghbi HY. Diseases of unstable repeat expansion: mechanisms and common principles. Nat. Rev. Genet. 6(10), 743-755 (2005).

2 Matos CA, De Macedo-Ribeiro S, Carvalho AL. Polyglutamine diseases: the special case of ataxin-3 and Machado-Joseph disease. Prog. Neurobiol. 95(1), 26-48 (2011).

3 Shao J, Diamond MI. Polyglutamine diseases: emerging concepts in pathogenesis and therapy. Hum. Mol. Genet. 16(2), R115-R123 (2007).

4 Mccolgan P, Tabrizi SJ. Huntington's disease: a clinical review. Eur. J. Neurol. doi:10.1111/ene.13413 (2017) (Epub ahead of print).

5 Ramachandra NB, Kusuma L. An understanding of spinocerebellar ataxia. Indian J. Med. Res. 141(2), 148-150 (2015).

6 Schols L, Bauer P, Schmidt T, Schulte T, Riess O. Autosomal dominant cerebellar ataxias: clinical features, genetics, and pathogenesis. Lancet Neurol. 3(5), 291-304 (2004).

7 Zoghbi HY, Orr HT. Glutamine repeats and neurodegeneration. Annu. Rev. Neurosci. 23, 217-247 (2000).

8 Ishikawa K, Fujigasaki H, Saegusa $\mathrm{H}$ et al. Abundant expression and cytoplasmic aggregations of [alpha] $1 \mathrm{~A}$ voltage-dependent calcium channel protein associated with neurodegeneration in spinocerebellar ataxia type 6. Hum. Mol. Genet. 8(7), 1185-1193 (1999).

9 Norremolle A, Sorensen SA, Fenger K, Hasholt L. Correlation between magnitude of CAG repeat length alterations and length of the paternal repeat in paternally inherited Huntington's disease. Clin. Genet. 47(3), 113-117 (1995).

10 Maciel P, Gaspar C, Destefano AL et al. Correlation between CAG repeat length and clinical features in Machado-Joseph disease. Am. J. Hum. Genet. 57(1), 54-61 (1995).

11 Ciechanover A, Brundin P. The ubiquitin proteasome system in neurodegenerative diseases: sometimes the chicken, sometimes the egg. Neuron 40(2), 427-446 (2003).

12 Liu H, Li X, Ning G et al. The Machado-Joseph disease deubiquitinase ataxin-3 regulates the stability and apoptotic function of $\mathrm{p} 53$. PLoS Biol. 14(11), e2000733 (2016).

13 Illuzzi JL, Vickers CA, Kmiec EB. Modifications of p 53 and the DNA damage response in cells expressing mutant form of the protein huntingtin. J. Mol. Neurosci. 45(2), 256-268 (2011).

14 Cohen-Carmon D, Meshorer E. Polyglutamine (polyQ) disorders: the chromatin connection. Nucleus 3(5), 433-441 (2012).

15 Bezprozvanny I. Role of inositol 1,4,5-trisphosphate receptors in pathogenesis of Huntington's disease and spinocerebellar ataxias. Neurochem. Res. 36(7), 1186-1197 (2011).

16 Bezprozvanny I. Calcium signaling and neurodegenerative diseases. Trends Mol. Med. 15(3), 89-100 (2009).

17 Li X, Liu H, Fischhaber PL, Tang TS. Toward therapeutic targets for SCA3: insight into the role of Machado-Joseph disease protein ataxin-3 in misfolded proteins clearance. Prog. Neurobiol. 132, 34-58 (2015).

18 Bauer PO, Nukina N. The pathogenic mechanisms of polyglutamine diseases and current therapeutic strategies. J. Neurochem. 110(6), 1737-1765 (2009).

- Comprehensive review of the pathogenesis of polyglutamine disorders.

19 Taylor JP, Tanaka F, Robitschek J et al. Aggresomes protect cells by enhancing the degradation of toxic polyglutamine-containing protein. Hum. Mol. Genet. 12(7), 749-757 (2003).

20 Li M, Chevalier-Larsen ES, Merry DE, Diamond MI. Soluble androgen receptor oligomers underlie pathology in a mouse model of spinobulbar muscular atrophy. J. Biol. Chem. 282(5), 3157-3164 (2007).

21 Arrasate M, Mitra S, Schweitzer ES, Segal MR, Finkbeiner S. Inclusion body formation reduces levels of mutant huntingtin and the risk of neuronal death. Nature 431(7010), 805-810 (2004).

22 Klement IA, Skinner PJ, Kaytor MD et al. Ataxin-1 nuclear localization and aggregation: role in polyglutamine-induced disease in SCA1 transgenic mice. Cell 95(1), 41-53 (1998).

23 Saudou F, Finkbeiner S, Devys D, Greenberg ME. Huntingtin acts in the nucleus to induce apoptosis but death does not correlate with the formation of intranuclear inclusions. Cell 95(1), 55-66 (1998).

- First report of the dissociation of inclusion bodies from the pathogenic process of Huntington's disease (HD). 
24 Adwan L, Zawia NH. Epigenetics: a novel therapeutic approach for the treatment of Alzheimer's disease. Pharmacol. Ther. 139(1), 41-50 (2013).

25 Klose RJ, Bird AP. Genomic DNA methylation: the mark and its mediators. Trends Biochem. Sci. 31(2), 89-97 (2006).

26 Jones PA. Functions of DNA methylation: islands, start sites, gene bodies and beyond. Nat. Rev. Genet. 13(7), 484-492 (2012).

27 Illingworth RS, Bird AP. CpG islands - ‘a rough guide’. FEBS Lett. 583(11), 1713-1720 (2009).

28 Thomas B, Matson S, Chopra V et al. A novel method for detecting 7-methyl guanine reveals aberrant methylation levels in Huntington disease. Anal. Biochem. 436(2), 112-120 (2013).

29 Guo JU, Su Y, Shin JH et al. Distribution, recognition and regulation of non-CpG methylation in the adult mammalian brain. Nat. Neurosci. 17(2), 215-222 (2014).

30 Ziller MJ, Gu H, Muller F et al. Charting a dynamic DNA methylation landscape of the human genome. Nature 500(7463), 477-481 (2013).

31 Flores K, Wolschin F, Corneveaux JJ, Allen AN, Huentelman MJ, Amdam GV. Genome-wide association between DNA methylation and alternative splicing in an invertebrate. BMC Genomics 13, 480 (2012).

32 Lyko F, Foret S, Kucharski R, Wolf S, Falckenhayn C, Maleszka R. The honey bee epigenomes: differential methylation of brain DNA in queens and workers. PLoS Biol. 8(11), e1000506 (2010).

33 Sherwani SI, Khan HA. Role of 5-hydroxymethylcytosine in neurodegeneration. Gene 570(1), 17-24 (2015).

34 Bernstein AI, Lin Y, Street RC et al. 5-Hydroxymethylation-associated epigenetic modifiers of Alzheimer's disease modulate Tau-induced neurotoxicity. Hum. Mol. Genet. 25(12), 2437-2450 (2016).

35 Szulwach KE, Li X, Li Y et al. 5-hmC-mediated epigenetic dynamics during postnatal neurodevelopment and aging. Nat. Neurosci. 14(12), 1607-1616 (2011).

36 Wang T, Wu H, Li Y et al. Subtelomeric hotspots of aberrant 5-hydroxymethylcytosine-mediated epigenetic modifications during reprogramming to pluripotency. Nat. Cell Biol. 15(6), 700-711 (2013).

37 Munzel M, Globisch D, Carell T. 5-Hydroxymethylcytosine, the sixth base of the genome. Angew. Chem. Int. Ed. Engl. 50(29), 6460-6468 (2011).

- Proposes hydroxymethylcytosine functions as an important epigenetic marker.

38 van den Hove DL, Chouliaras L, Rutten BP. The role of 5-hydroxymethylcytosine in aging and Alzheimer's disease: current status and prospects for future studies. Curr. Alzheimer Res. 9(5), 545-549 (2012).

39 Marques SC, Oliveira CR, Pereira CM, Outeiro TF. Epigenetics in neurodegeneration: a new layer of complexity. Prog. Neuropsychopharmacol. Biol. Psychiatry 35(2), 348-355 (2011).

40 Xu Z, Li H, Jin P. Epigenetics-based therapeutics for neurodegenerative disorders. Curr. Transl. Geriatr. Exp. Gerontol. Rep. 1(4), 229-236 (2012).

41 Graff J, Kim D, Dobbin MM, Tsai LH. Epigenetic regulation of gene expression in physiological and pathological brain processes. Physiol. Rev. 91(2), 603-649 (2011).

42 Lee J, Hwang YJ, Shin JY et al. Epigenetic regulation of cholinergic receptor M1 (CHRM1) by histone H3K9me3 impairs Ca(2+) signaling in Huntington's disease. Acta Neuropathol. 125(5), 727-739 (2013).

43 Langst G, Manelyte L. Chromatin remodelers: from function to dysfunction. Genes 6(2), 299-324 (2015).

44 Krol J, Loedige I, Filipowicz W. The widespread regulation of microRNA biogenesis, function and decay. Nat. Rev. Genet. 11(9), 597-610 (2010).

45 Suzuki T, Nagano Y, Matsuura A et al. Novel histone deacetylase inhibitors: design, synthesis, enzyme inhibition, and binding mode study of SAHA-based non-hydroxamates. Bioorg. Med. Chem. Lett. 13(24), $4321-4326$ (2003).

46 Cao JX, Zhang HP, Du LX. [Influence of environmental factors on DNA methylation]. Yi Chuan 35(7), 839-846 (2013).

47 Scott GK, Mattie MD, Berger CE, Benz SC, Benz CC. Rapid alteration of microRNA levels by histone deacetylase inhibition. Cancer Res. 66(3), 1277-1281 (2006).

48 Saito Y, Liang G, Egger G et al. Specific activation of microRNA-127 with downregulation of the proto-oncogene BCL6 by chromatin-modifying drugs in human cancer cells. Cancer Cell 9(6), 435-443 (2006).

49 Chen BF, Gu S, Suen YK, Li L, Chan WY. microRNA-199a-3p, DNMT3A, and aberrant DNA methylation in testicular cancer. Epigenetics 9(1), 119-128 (2014).

50 Fabbri M, Garzon R, Cimmino A et al. MicroRNA-29 family reverts aberrant methylation in lung cancer by targeting DNA methyltransferases 3A and 3B. Proc. Natl Acad. Sci. USA 104(40), 15805-15810 (2007).

51 Szulwach KE, Li X, Smrt RD et al. Cross talk between microRNA and epigenetic regulation in adult neurogenesis. J. Cell Biol. 189(1), 127-141 (2010).

52 Liu C, Teng ZQ, Santistevan NJ et al. Epigenetic regulation of miR-184 by MBD1 governs neural stem cell proliferation and differentiation. Cell Stem Cell 6(5), 433-444 (2010). 
53 Du J, Patel DJ. Structural biology-based insights into combinatorial readout and crosstalk among epigenetic marks. Biochim. Biophys. Acta 1839(8), 719-727 (2014)

54 Thomas EA. DNA methylation in Huntington's disease: implications for transgenerational effects. Neurosci. Lett. 625, 34-39 (2016).

$55 \mathrm{Ng}$ CW, Yildirim F, Yap YS et al. Extensive changes in DNA methylation are associated with expression of mutant huntingtin. Proc. Natl Acad. Sci. USA 110(6), 2354-2359 (2013).

\section{- First study on the genome-wide DNA methylation in HD cell models.}

56 Jia H, Morris CD, Williams RM, Loring JF, Thomas EA. HDAC inhibition imparts beneficial transgenerational effects in Huntington's disease mice via altered DNA and histone methylation. Proc. Natl Acad. Sci. USA 112(1), E56-E64 (2015).

57 Wang F, Yang Y, Lin X et al. Genome-wide loss of 5-hmC is a novel epigenetic feature of Huntington's disease. Hum. Mol. Genet. 22(18), 3641-3653 (2013).

- First study on the genome-wide 5-hydroxymethylation in HD mice models.

58 Villar-Menendez I, Blanch M, Tyebji S et al. Increased 5-methylcytosine and decreased 5-hydroxymethylcytosine levels are associated with reduced striatal A2AR levels in Huntington's disease. Neuromolecular Med. 15(2), 295-309 (2013).

59 De Souza RA, Islam SA, McEwen LM et al. DNA methylation profiling in human Huntington's disease brain. Hum. Mol. Genet. 25(10), 2013-2030 (2016).

60 Bai G, Cheung I, Shulha HP et al. Epigenetic dysregulation of hairy and enhancer of split 4 (HES4) is associated with striatal degeneration in postmortem Huntington brains. Hum. Mol. Genet. 24(5), 1441-1456 (2015).

61 Reik W, Maher ER, Morrison PJ, Harding AE, Simpson SA. Age at onset in Huntington's disease and methylation at D4S95. J. Med. Genet. 30(3), 185-188 (1993).

62 Flanagan JM, Popendikyte V, Pozdniakovaite N et al. Intra- and interindividual epigenetic variation in human germ cells. Am. J. Hum. Genet. 79(1), 67-84 (2006).

63 Narayanan M, Huynh JL, Wang K et al. Common dysregulation network in the human prefrontal cortex underlies two neurodegenerative diseases. Mol. Syst. Biol. 10, 743 (2014).

64 Thomas EA, Coppola G, Desplats PA et al. The HDAC inhibitor $4 \mathrm{~b}$ ameliorates the disease phenotype and transcriptional abnormalities in Huntington's disease transgenic mice. Proc. Natl Acad. Sci. USA 105(40), 15564-15569 (2008).

65 Tang B, Seredenina T, Coppola G et al. Gene expression profiling of R6/2 transgenic mice with different CAG repeat lengths reveals genes associated with disease onset and progression in Huntington's disease. Neurobiol. Dis. 42(3), 459-467 (2011).

66 Schmitt I, Evert BO, Khazneh H, Klockgether T, Wuellner U. The human MJD gene: genomic structure and functional characterization of the promoter region. Gene 314, 81-88 (2003).

67 Wang C, Peng H, Li J et al. Alteration of methylation status in the ATXN3 gene promoter region is linked to the SCA3/MJD. Neurobiol. Aging 53, 192.e5-192.e10 (2017).

68 Dick KJ, Nelson CP, Braund PS, Goodall AH, Samani NJ. Genome wide methylation analysis in coronary artery disease. Heart 97 , A42-A42 (2011).

69 Aguiar J, Santurlidis S, Nowok J et al. Identification of the physiological promoter for spinocerebellar ataxia 2 gene reveals a CpG island for promoter activity situated into the exon 1 of this gene and provides data about the origin of the nonmethylated state of these types of islands. Biochem. Biophys. Res. Commun. 254(2), 315-318 (1999).

70 Laffita-Mesa JM, Bauer PO, Kouri V et al. Epigenetics DNA methylation in the core ataxin-2 gene promoter: novel physiological and pathological implications. Hum. Genet. 131(4), 625-638 (2012).

71 Dion V, Lin Y, Hubert L Jr, Waterland RA, Wilson JH. Dnmt1 deficiency promotes CAG repeat expansion in the mouse germline. Hum. Mol. Genet. 17(9), 1306-1317 (2008).

72 Libby RT, Hagerman KA, Pineda VV et al. CTCF cis-regulates trinucleotide repeat instability in an epigenetic manner: a novel basis for mutational hot spot determination. PLoS Genet. 4(11), e1000257 (2008).

73 Moumne L, Betuing S, Caboche J. Multiple aspects of gene dysregulation in Huntington's disease. Front. Neurol. 4, 127 (2013).

74 Sadri-Vakili G, Bouzou B, Benn CL et al. Histones associated with downregulated genes are hypo-acetylated in Huntington's disease models. Hum. Mol. Genet. 16(11), 1293-1306 (2007).

75 Pena-Altamira LE, Polazzi E, Monti B. Histone post-translational modifications in Huntington's and Parkinson's diseases. Curr. Pharm. Des. 19(28), 5085-5092 (2013).

76 Steffan JS, Bodai L, Pallos J et al. Histone deacetylase inhibitors arrest polyglutamine-dependent neurodegeneration in Drosophila. Nature 413(6857), 739-743 (2001).

- Report on the possibility of using histone deacetylase inhibitors to treat polyglutamine disorders.

77 Steffan JS, Kazantsev A, Spasic-Boskovic O et al. The Huntington's disease protein interacts with $\mathrm{p} 53$ and CREB-binding protein and represses transcription. Proc. Natl Acad. Sci. USA 97(12), 6763-6768 (2000).

78 Ferrante RJ, Kubilus JK, Lee J et al. Histone deacetylase inhibition by sodium butyrate chemotherapy ameliorates the neurodegenerative phenotype in Huntington's disease mice. J. Neurosci. 23(28), 9418-9427 (2003). 
79 Gardian G, Browne SE, Choi DK et al. Neuroprotective effects of phenylbutyrate in the N171-82Q transgenic mouse model of Huntington's disease. J. Biol. Chem. 280(1), 556-563 (2005).

80 Yeh HH, Young D, Gelovani JG et al. Histone deacetylase class II and acetylated core histone immunohistochemistry in human brains with Huntington's disease. Brain Res. 1504, 16-24 (2013).

81 Dompierre JP, Godin JD, Charrin BC et al. Histone deacetylase 6 inhibition compensates for the transport deficit in Huntington's disease by increasing tubulin acetylation. J. Neurosci. 27(13), 3571-3583 (2007).

82 McFarland KN, Das S, Sun TT et al. Genome-wide histone acetylation is altered in a transgenic mouse model of Huntington's disease. PLoS ONE 7(7), e41423 (2012).

83 Anderson AN, Roncaroli F, Hodges A, Deprez M, Turkheimer FE. Chromosomal profiles of gene expression in Huntington's disease. Brain 131(Pt 2), 381-388 (2008).

84 Igarashi S, Morita H, Bennett KM et al. Inducible PC12 cell model of Huntington's disease shows toxicity and decreased histone acetylation. Neuroreport 14(4), 565-568 (2003).

85 Chou AH, Chen SY, Yeh TH, Weng YH, Wang HL. HDAC inhibitor sodium butyrate reverses transcriptional downregulation and ameliorates ataxic symptoms in a transgenic mouse model of SCA3. Neurobiol. Dis. 41(2), 481-488 (2011).

86 Chou AH, Chen YL, Hu SH, Chang YM, Wang HL. Polyglutamine-expanded ataxin-3 impairs long-term depression in Purkinje neurons of SCA3 transgenic mouse by inhibiting HAT and impairing histone acetylation. Brain Res. 1583, $220-229$ (2014).

87 Li SH, Li XJ. Huntingtin-protein interactions and the pathogenesis of Huntington's disease. Trends Genet. 20(3), 146-154 (2004).

88 Harjes P, Wanker EE. The hunt for huntingtin function: interaction partners tell many different stories. Trends Biochem. Sci. 28(8), 425-433 (2003).

89 Ryu H, Lee J, Hagerty SW et al. ESET/SETDB1 gene expression and histone H3 (K9) trimethylation in Huntington's disease. Proc. Natl Acad. Sci. USA 103(50), 19176-19181 (2006).

90 Ferrante RJ, Ryu H, Kubilus JK et al. Chemotherapy for the brain: the antitumor antibiotic mithramycin prolongs survival in a mouse model of Huntington's disease. J. Neurosci. 24(46), 10335-10342 (2004).

91 Kazantsev A, Preisinger E, Dranovsky A, Goldgaber D, Housman D. Insoluble detergent-resistant aggregates form between pathological and nonpathological lengths of polyglutamine in mammalian cells. Proc. Natl Acad. Sci. USA 96(20), 11404-11409 (1999).

92 Nucifora FC Jr, Sasaki M, Peters MF et al. Interference by huntingtin and atrophin-1 with CBP-mediated transcription leading to cellular toxicity. Science 291(5512), 2423-2428 (2001).

93 Stack EC, Del Signore SJ, Luthi-Carter R et al. Modulation of nucleosome dynamics in Huntington's disease. Hum. Mol. Genet. 16(10), 1164-1175 (2007).

94 Taylor JP, Taye AA, Campbell C, Kazemi-Esfarjani P, Fischbeck KH, Min KT. Aberrant histone acetylation, altered transcription, and retinal degeneration in a Drosophila model of polyglutamine disease are rescued by CREB-binding protein. Genes Dev. 17(12), 1463-1468 (2003).

95 Klevytska AM, Tebbenkamp AT, Savonenko AV, Borchelt DR. Partial depletion of CREB-binding protein reduces life expectancy in a mouse model of Huntington disease. J. Neuropathol. Exp. Neurol. 69(4), 396-404 (2010).

96 Wang H, Wang L, Erdjument-Bromage H et al. Role of histone H2A ubiquitination in Polycomb silencing. Nature 431(7010), 873-878 (2004).

97 Kim MO, Chawla P, Overland RP, Xia E, Sadri-Vakili G, Cha JH. Altered histone monoubiquitylation mediated by mutant huntingtin induces transcriptional dysregulation. J. Neurosci. 28(15), 3947-3957 (2008).

98 Bett JS, Benn CL, Ryu KY, Kopito RR, Bates GP. The polyubiquitin Ubc gene modulates histone H2A monoubiquitylation in the R6/2 mouse model of Huntington's disease. J. Cell. Mol. Med. 13(8B), 2645-2657 (2009).

99 Gehrking KM, Andresen JM, Duvick L, Lough J, Zoghbi HY, Orr HT. Partial loss of Tip60 slows mid-stage neurodegeneration in a spinocerebellar ataxia type 1 (SCA1) mouse model. Hum. Mol. Genet. 20(11), 2204-2212 (2011).

100 Li F, Macfarlan T, Pittman RN, Chakravarti D. Ataxin-3 is a histone-binding protein with two independent transcriptional corepressor activities. J. Biol. Chem. 277(47), 45004-45012 (2002).

101 Fiesel FC, Schurr C, Weber SS, Kahle PJ. TDP-43 knockdown impairs neurite outgrowth dependent on its target histone deacetylase 6. Mol. Neurodegener. 6, 64 (2011).

102 Fiesel FC, Voigt A, Weber SS et al. Knockdown of transactive response DNA-binding protein (TDP-43) downregulates histone deacetylase 6. EMBO J. 29(1), 209-221 (2010).

103 Helmlinger D, Hardy S, Eberlin A, Devys D, Tora L. Both normal and polyglutamine- expanded ataxin-7 are components of TFTC-type GCN5 histone acetyltransferase-containing complexes. Biochem. Soc. Symp. 73, 155-163 (2006).

104 McCullough SD, Grant PA. Histone acetylation, acetyltransferases, and ataxia-alteration of histone acetylation and chromatin dynamics is implicated in the pathogenesis of polyglutamine-expansion disorders. Adv. Protein Chem. Struct. Biol. 79, 165-203 (2010).

105 Lau P, de Strooper B. Dysregulated microRNAs in neurodegenerative disorders. Semin. Cell Dev. Biol. 21(7), 768-773 (2010). 
106 Karnati HK, Panigrahi MK, Gutti RK, Greig NH, Tamargo IA. miRNAs: key players in neurodegenerative disorders and epilepsy. J. Alzheimers Dis. 48(3), 563-580 (2015).

107 Qiu L, Tan EK, Zeng L. microRNAs and neurodegenerative diseases. Adv. Exp. Med. Biol. 888, 85-105 (2015).

108 Gupta S, Verma S, Mantri S, Berman NE, Sandhir R. Targeting microRNAs in prevention and treatment of neurodegenerative disorders. Drug Dev. Res. 76(7), 397-418 (2015).

109 Cao DD, Li L, Chan WY. MicroRNAs: key regulators in the central nervous system and their implication in neurological diseases. Int. J. Mol. Sci. 17(6), pii:E842 (2016).

110 Meza-Sosa KF, Valle-Garcia D, Pedraza-Alva G, Perez-Martinez L. Role of microRNAs in central nervous system development and pathology. J. Neurosci. Res. 90(1), 1-12 (2012).

111 Viswambharan V, Thanseem I, Vasu MM, Poovathinal SA, Anitha A. miRNAs as biomarkers of neurodegenerative disorders. Biomark. Med. 11(2), 151-167 (2017).

112 Tan H, Xu Z, Jin P. Role of noncoding RNAs in trinucleotide repeat neurodegenerative disorders. Exp. Neurol. 235(2), 469-475 (2012).

113 Bilen J, Liu N, Bonini NM. A new role for microRNA pathways: modulation of degeneration induced by pathogenic human disease proteins. Cell Cycle 5(24), 2835-2838 (2006).

114 Bilen J, Liu N, Burnett BG, Pittman RN, Bonini NM. MicroRNA pathways modulate polyglutamine-induced neurodegeneration. Mol. Cell 24(1), 157-163 (2006).

-. First evidence of the miRNA pathways plays protective roles in the polyglutamine neurodegeneration.

115 Hebert SS, De Strooper B. Alterations of the microRNA network cause neurodegenerative disease. Trends Neurosci. 32(4), 199-206 (2009).

116 Schaefer A, O'Carroll D, Tan CL et al. Cerebellar neurodegeneration in the absence of microRNAs. J. Exp. Med. 204(7), 1553-1558 (2007).

117 Liu N, Landreh M, Cao K et al. The microRNA miR-34 modulates ageing and neurodegeneration in Drosophila. Nature 482(7386), 519-523 (2012).

118 Savas JN, Makusky A, Ottosen S et al. Huntington's disease protein contributes to RNA-mediated gene silencing through association with Argonaute and P bodies. Proc. Natl Acad. Sci. USA 105(31), 10820-10825 (2008).

119 Buckley NJ, Johnson R. New insights into non-coding RNA networks in Huntington's disease. Exp. Neurol. 231(2), 191-194 (2011).

120 Marti E, Pantano L, Banez-Coronel M et al. A myriad of miRNA variants in control and Huntington's disease brain regions detected by massively parallel sequencing. Nucleic Acids Res. 38(20), 7219-7235 (2010).

121 Kocerha J, Xu Y, Prucha MS, Zhao D, Chan AW. microRNA-128a dysregulation in transgenic Huntington's disease monkeys. Mol. Brain 7, 46 (2014).

122 Sinha M, Ghose J, Das E, Bhattarcharyya NP. Altered microRNAs in STHdh(Q111)/Hdh(Q111) cells: miR-146a targets TBP. Biochem. Biophys. Res. Commun. 396(3), 742-747 (2010).

123 Ghose J, Sinha M, Das E, Jana NR, Bhattacharyya NP. Regulation of miR-146a by RelA/NFkB and p53 in STHdh(Q111)/Hdh(Q111) cells, a cell model of Huntington's disease. PLoS ONE 6(8), e23837 (2011).

124 Packer AN, Xing Y, Harper SQ, Jones L, Davidson BL. The bifunctional microRNA miR-9/miR-9* regulates REST and CoREST and is downregulated in Huntington's disease. J. Neurosci. 28(53), 14341-14346 (2008).

125 Kunkanjanawan T, Carter RL, Prucha MS, Yang J, Parnpai R, Chan AW. miR-196a ameliorates cytotoxicity and cellular phenotype in transgenic Huntington's disease monkey neural cells. PLoS ONE 11(9), e0162788 (2016).

126 Hoss AG, Kartha VK, Dong X et al. MicroRNAs located in the Hox gene clusters are implicated in Huntington's disease pathogenesis. PLoS Genet. 10(2), e1004188 (2014).

127 Jin J, Cheng Y, Zhang Y et al. Interrogation of brain miRNA and mRNA expression profiles reveals a molecular regulatory network that is perturbed by mutant huntingtin. J. Neurochem. 123(4), 477-490 (2012).

128 Johnson R, Buckley NJ. Gene dysregulation in Huntington's disease: REST, microRNAs and beyond. Neuromolecular Med. 11(3), 183-199 (2009).

129 Johnson R, Zuccato C, Belyaev ND, Guest DJ, Cattaneo E, Buckley NJ. A microRNA-based gene dysregulation pathway in Huntington's disease. Neurobiol. Dis. 29(3), 438-445 (2008).

130 Lee ST, Chu K, Im WS et al. Altered microRNA regulation in Huntington's disease models. Exp. Neurol. 227(1), 172-179 (2011).

131 Zuccato C, Tartari M, Crotti A et al. Huntingtin interacts with REST/NRSF to modulate the transcription of NRSE-controlled neuronal genes. Nat. Genet. 35(1), 76-83 (2003).

132 Buckley NJ, Johnson R, Zuccato C, Bithell A, Cattaneo E. The role of REST in transcriptional and epigenetic dysregulation in Huntington's disease. Neurobiol. Dis. 39(1), 28-39 (2010).

133 Ooi L, Wood IC. Chromatin crosstalk in development and disease: lessons from REST. Nat. Rev. Genet. 8(7), 544-554 (2007).

134 Gascon E, Gao FB. Cause or effect: misregulation of microRNA pathways in neurodegeneration. Front. Neurosci. 6, 48 (2012). 
135 Koscianska E, Krzyzosiak WJ. Current understanding of the role of microRNAs in spinocerebellar ataxias. Cerebellum Ataxias 1, 7 (2014).

136 McCann C, Holohan EE, Das $S$ et al. The Ataxin-2 protein is required for microRNA function and synapse-specific long-term olfactory habituation. Proc. Natl Acad. Sci. USA 108(36), E655-E662 (2011).

137 Carmona V, Cunha-Santos J, Onofre I et al. Unravelling endogenous microRNA system dysfunction as a new pathophysiological mechanism in Machado-Joseph disease. Mol. Ther. 25(4), 1038-1055 (2017).

138 Roshan R, Ghosh T, Gadgil M, Pillai B. Regulation of BACE1 by miR-29a/b in a cellular model of spinocerebellar ataxia 17. RNA Biol. 9(6), 891-899 (2012).

139 Persengiev S, Kondova I, Otting N, Koeppen AH, Bontrop RE. Genome-wide analysis of miRNA expression reveals a potential role for miR-144 in brain aging and spinocerebellar ataxia pathogenesis. Neurobiol. Aging 32(12), 2316.e17-2316.e27 (2011).

140 Rodriguez-Lebron E, Liu G, Keiser M, Behlke MA, Davidson BL. Altered purkinje cell miRNA expression and SCA1 pathogenesis. Neurobiol. Dis. 54, 456-463 (2013).

141 Lee Y, Samaco RC, Gatchel JR, Thaller C, Orr HT, Zoghbi HY. miR-19, miR-101 and miR-130 co-regulate ATXN1 levels to potentially modulate SCA1 pathogenesis. Nat. Neurosci. 11(10), 1137-1139 (2008).

142 Lukiw WJ. Micro-RNA speciation in fetal, adult and Alzheimer's disease hippocampus. Neuroreport 18(3), 297-300 (2007).

143 Sethi P, Lukiw WJ. Micro-RNA abundance and stability in human brain: specific alterations in Alzheimer's disease temporal lobe neocortex. Neurosci. Lett. 459(2), 100-104 (2009).

144 Hebert SS, Horre K, Nicolai L et al. Loss of microRNA cluster miR-29a/b-1 in sporadic Alzheimer's disease correlates with increased BACE1/beta-secretase expression. Proc. Natl Acad. Sci. USA 105(17), 6415-6420 (2008).

145 Wang X, Liu P, Zhu H et al. miR-34a, a microRNA up-regulated in a double transgenic mouse model of Alzheimer's disease, inhibits bcl2 translation. Brain Res. Bull. 80(4-5), 268-273 (2009).

146 Yan R, Vassar R. Targeting the beta secretase BACE1 for Alzheimer's disease therapy. Lancet Neurol. 13(3), 319-329 (2014).

147 Shi YT, Huang FZ, Tang BS et al. MicroRNA profiling in the serums of SCA3/MJD patients. Int. J. Neurosci. 124(2), 97-101 (2014).

148 Hoss AG, Lagomarsino VN, Frank S, Hadzi TC, Myers RH, Latourelle JC. Study of plasma-derived miRNAs mimic differences in Huntington's disease brain. Mov. Disord. 30(14), 1961-1964 (2015).

149 Gaughwin PM, Ciesla M, Lahiri N, Tabrizi SJ, Brundin P, Bjorkqvist M. Hsa-miR-34b is a plasma-stable microRNA that is elevated in pre-manifest Huntington's disease. Hum. Mol. Genet. 20(11), 2225-2237 (2011).

150 Hoss AG, Labadorf A, Latourelle JC et al. miR-10b-5p expression in Huntington's disease brain relates to age of onset and the extent of striatal involvement. BMC Med. Genomics 8, 10 (2015).

151 Diez-Planelles C, Sanchez-Lozano P, Crespo MC et al. Circulating microRNAs in Huntington's disease: emerging mediators in metabolic impairment. Pharmacol. Res. 108, 102-110 (2016).

152 Seto AG. The road toward microRNA therapeutics. Int. J. Biochem. Cell Biol. 42(8), 1298-1305 (2010).

153 Ebert MS, Neilson JR, Sharp PA. MicroRNA sponges: competitive inhibitors of small RNAs in mammalian cells. Nat. Methods 4(9), 721-726 (2007).

154 Sapru MK, Yates JW, Hogan S, Jiang L, Halter J, Bohn MC. Silencing of human alpha-synuclein in vitro and in rat brain using lentiviral-mediated RNAi. Exp. Neurol. 198(2), 382-390 (2006).

$155 \mathrm{Hu}$ J, Liu J, Corey DR. Allele-selective inhibition of huntingtin expression by switching to an miRNA-like RNAi mechanism. Chem. Biol. 17(11), 1183-1188 (2010).

156 Fiszer A, Mykowska A, Krzyzosiak WJ. Inhibition of mutant huntingtin expression by RNA duplex targeting expanded CAG repeats. Nucleic Acids Res. 39(13), 5578-5585 (2011).

157 Boudreau RL, McBride JL, Martins I et al. Nonallele-specific silencing of mutant and wild-type huntingtin demonstrates therapeutic efficacy in Huntington's disease mice. Mol. Ther. 17(6), 1053-1063 (2009).

158 McBride JL, Boudreau RL, Harper SQ et al. Artificial miRNAs mitigate shRNA-mediated toxicity in the brain: implications for the therapeutic development of RNAi. Proc. Natl Acad. Sci. USA 105(15), 5868-5873 (2008).

- Proposes that miRNA-based approaches may provide more appropriate biological tools for expressing inhibitory RNAs in the brain.

159 Cheng PH, Li CL, Chang YF et al. miR-196a ameliorates phenotypes of Huntington disease in cell, transgenic mouse, and induced pluripotent stem cell models. Am. J. Hum. Genet. 93(2), 306-312 (2013).

160 Keiser MS, Geoghegan JC, Boudreau RL, Lennox KA, Davidson BL. RNAi or overexpression: alternative therapies for spinocerebellar ataxia type 1. Neurobiol. Dis. 56, 6-13 (2013).

161 Keiser MS, Boudreau RL, Davidson BL. Broad therapeutic benefit after RNAi expression vector delivery to deep cerebellar nuclei: implications for spinocerebellar ataxia type 1 therapy. Mol. Ther. 22(3), 588-595 (2014).

162 Rodriguez-Lebron E, Costa MD, Luna-Cancalon K et al. Silencing mutant ATXN3 expression resolves molecular phenotypes in SCA3 transgenic mice. Mol. Ther. 21(10), 1909-1918 (2013). 
163 Costa Mdo C, Luna-Cancalon K, Fischer S et al. Toward RNAi therapy for the polyglutamine disease Machado-Joseph disease. Mol. Ther. 21(10), 1898-1908 (2013).

164 Yao B, Jin P. Unlocking epigenetic codes in neurogenesis. Genes Dev. 28(12), 1253-1271 (2014).

165. Sinha M, Ghose J, Bhattarcharyya NP et al. Micro RNA -214,-150,-146a and-125b target Huntingtin gene. RNA Biol.8(6), 1005-1021 (2011).

166. Das E, Jana NR, Bhattacharyya NP.Delayed cell cycle progression in STHdh(Q111)/Hdh(Q111) cells, a cell model for Huntington's disease mediated by microRNA-19a, microRNA-146a and microRNA-432. MicroRNA4(2), 86-100 (2015).

167. Huang F, Zhang L, Long Z et al. miR-25 alleviates polyQ-mediated cytotoxicity by silencing ATXN3. FEBS Lett. 588(24), 4791-4798 (2014).

168. Reinhardt A, Feuillette S, Cassar M et al. Lack of miRNA misregulation at earlyp athological stages in Drosophila neurodegenerative disease models. Front. Genet. 3, 226 (2012). 
(

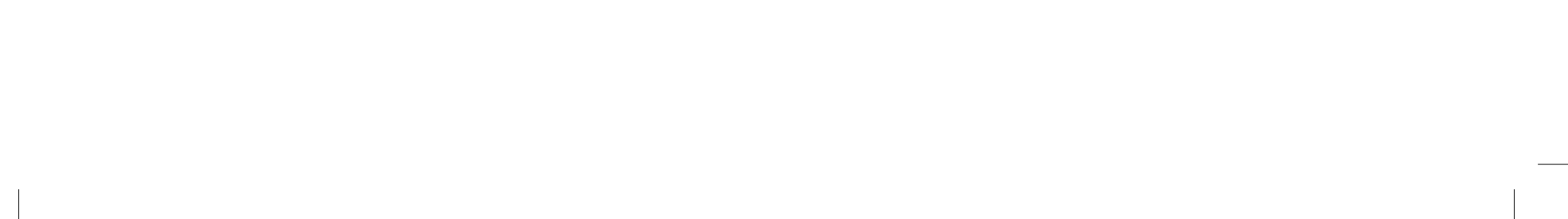




\title{
Maternal alcohol consumption and offspring DNA methylation: findings from six general population-based birth cohorts
}

\author{
Gemma C Sharp ${ }^{\ddagger 1,2,3}$, Ryan Arathimos ${ }^{*} \neq, 1,2$, Sarah E Reese ${ }^{4}$, Christian M Page ${ }^{5,6}$, Janine \\ Felix $^{7,8,9}$, Leanne K Küpers ${ }^{1,2,10}$, Sheryl L Rifas-Shiman ${ }^{11}$, Chunyu Liu ${ }^{12,13,14}$, The Cohorts for \\ Heart and Aging Research in Genomic Epidemiology plus (CHARGE +) methylation alcohol \\ working group, Kimberley Burrows ${ }^{1,2}$, Shanshan Zhao ${ }^{4}$, Maria C Magnus ${ }^{1,2,5}$, Liesbeth \\ Duijts $^{7,15,16}$, Eva Corpeleijn ${ }^{10}$, Dawn L DeMeo ${ }^{17}$, Augusto Litonjua ${ }^{17}$, Andrea Baccarelli ${ }^{18}$, \\ Marie-France Hivert ${ }^{11,19}$, Emily Oken ${ }^{11}$, Harold Snieder ${ }^{10}$, Vincent Jaddoe ${ }^{7,8,9}$, Wenche \\ Nystad $^{5}$, Stephanie J London ${ }^{4}$, Caroline L Relton ${ }^{1,2}$ \& Luisa Zuccolo ${ }^{1,2}$ \\ ${ }^{1} \mathrm{MRC}$ Integrative Epidemiology Unit, University of Bristol, Bristol, BS8 2BN, UK \\ ${ }^{2}$ School of Social \& Community Medicine, University of Bristol, Bristol, BS8 2BN, UK \\ ${ }^{3}$ School of Oral \& Dental Sciences, University of Bristol, Bristol, UK \\ ${ }^{4}$ Division of Intramural Research, Department of Health \& Human Services, National Institute of Environmental Health Sciences, \\ National Institutes of Health, Research Triangle Park, NC, USA \\ ${ }^{5}$ Division for Mental \& Physical Health, Department of Non-Communicable Diseases, Norwegian Institute of Public Health, Oslo, \\ Norway \\ ${ }^{6}$ Oslo Centre for Biostatistics \& Epidemiology, Oslo University Hospital, Oslo, Norway \\ ${ }^{7}$ The Generation R Study Group, Erasmus MC, University Medical Centre Rotterdam, Rotterdam, The Netherlands \\ ${ }^{8}$ Department of Epidemiology, Erasmus MC, University Medical Centre Rotterdam, Rotterdam, The Netherlands \\ ${ }^{9}$ Department of Pediatrics, Erasmus MC, University Medical Centre Rotterdam, Rotterdam, The Netherlands \\ ${ }^{10}$ Department of Epidemiology, University of Groningen, University Medical Center Groningen, Groningen, The Netherlands \\ ${ }^{11}$ Department of Population Medicine, Harvard Medical School, Harvard Pilgrim Health Care Institute, Boston, MA, USA \\ ${ }^{12}$ The Framingham Heart Study, Framingham, MA, USA \\ ${ }^{13}$ The Population Sciences Branch, Division of Intramural Research, National Heart, Lung, \& Blood Institute, Bethesda, MD, USA \\ ${ }^{14}$ Department of Biostatistics, Boston University School of Public Health, 715 Albany St, Boston, MA, USA \\ ${ }^{15}$ Department of Pediatrics, Division of Respiratory Medicine \& Allergology, Erasmus MC, University Medical Centre Rotterdam, \\ Rotterdam, The Netherlands \\ ${ }^{16}$ Department of Pediatrics, Division of Neonatology, Erasmus MC, University Medical Centre Rotterdam, Rotterdam, The \\ Netherlands \\ ${ }^{17}$ Channing Division of Network Medicine, Brigham \& Women's Hospital, Harvard Medical School, Boston, MA, USA \\ ${ }^{18}$ Laboratory of Precision Environmental Biosciences, Columbia University Mailman School of Public Health, New York, NY, USA \\ ${ }^{19}$ Diabetes Unit, Massachusetts General Hospital, Boston, MA, USA \\ * Author for correspondence: ryan.arathimos@bristol.ac.uk \\ Authors contributed equally
}

\begin{abstract}
Aim: Alcohol consumption during pregnancy is sometimes associated with adverse outcomes in offspring, potentially mediated by epigenetic modifications. We aimed to investigate genome-wide DNA methylation in cord blood of newborns exposed to alcohol in utero. Materials \& methods: We meta-analyzed information from six population-based birth cohorts within the Pregnancy and Childhood Epigenetics consortium. Results: We found no strong evidence of association at either individual $\mathrm{CpGs}$ or across larger regions of the genome. Conclusion: Our findings suggest no association between maternal alcohol consumption and offspring cord blood DNA methylation. This is in stark contrast to the multiple strong associations previous studies have found for maternal smoking, which is similarly socially patterned. However, it is possible that a combination of a larger sample size, higher doses, different timings of exposure, exploration of a different tissue and a more global assessment of genomic DNA methylation might show evidence of association.
\end{abstract}

First draft submitted: 3 August 2017; Accepted for publication: 27 September 2017; Published online: 27 November 2017

Keywords: alcohol $\bullet$ cord blood $\bullet$ DNA methylation $\bullet$ epidemiology $\bullet$ epigenetics $\bullet$ meta-analysis $\bullet$ PACE consortium - pregnancy 
It is well known that heavy alcohol consumption during pregnancy can cause Fetal Alcohol Spectrum Disorders (FASD), a spectrum of disorders characterized by a continuum of structural and neurodevelopmental abnormalities, with Fetal Alcohol Syndrome at the more severe end of the spectrum [1-3]. The severity of FASD appears to depend largely on the timing, dose and frequency of exposure to alcohol, with heavy exposure in the latter half of the first trimester being associated with the most severe effect $[4,5]$. However, in the general population most pregnant women do not drink at the doses required to cause FASD. For example, in population-based studies from Ireland, the UK, Australia and New Zealand, around 70\% (range: 67-77\%) of women who reported drinking in the first trimester consumed seven units or fewer per week, which is considered light-to-moderate consumption. In the second trimester, nearly all women who drank (range 99-100\%) consumed seven units or fewer per week [6]. Evidence of an effect of light-to-moderate levels of prenatal alcohol exposure is sparse and inconsistent. Although the majority of systematic reviews and studies published after these reviews have not found convincing evidence of association between light-to-moderate drinking and adverse offspring health and neurodevelopment [7-14], a recent comprehensive review of prospective studies found suggestive evidence of an association between mothers consuming up to four UK units of alcohol per week and babies born small-for-gestational age or preterm [15]. Furthermore, results from quasi-experimental study designs, which are more robust to the presence of confounding by parental socio-economic factors, have shown some evidence of effect of (mostly light-to-moderate) maternal alcohol consumption on offspring cognition and behavior [16-18]. The inconsistency of findings may be explained by the failure to adequately control for certain confounding factors, such as socioeconomic position, diet and ethnicity [19], which affect offspring outcomes both prenatally and postnatally, and could therefore bias any potentially small effect of light-to-moderate drinking in pregnancy [20]. Suggestions of harm from these later studies, together with findings from animal experiments, have prompted the UK Chief Medical Officer to recently revise the guidelines for alcohol drinking in pregnancy to recommend abstention, based on the precautionary principle [21].

Whether there is a causal association between light-to-moderate drinking in pregnancy and children's health outcomes is an important question. Identifying a possible biological pathway showing effects at birth would be a first step toward providing an answer. Currently, precise biological mechanisms underlying potential adverse effects of prenatal alcohol exposure are unknown. However, epigenetic modifications have been suggested as one such potential mediator, with some evidence that this is the case for prenatal exposure to smoking [22-24].

Animal studies suggest that alcohol exposure affects DNA methylation levels both globally, through its antagonistic effect on methyl donors such as folate [25,26], and in a gene-specific fashion [27,28]. Mouse pups exposed to alcohol during the highly epigenetically-sensitive intrauterine period show dose- and timing-specific epigenetic effects, including DNA methylation effects that correlate with long-lasting changes in gene expression and could potentially drive offspring adverse outcomes [29].

Similar experiments are impossible in humans for obvious ethical reasons. However, there is some evidence that treatment with a low physiologically relevant dose of ethanol induces genome-wide changes in DNA methylation in human embryonic stem cells [30]. In addition, a recent observational study of 110 children with FASD and 96 controls found genome-wide differences in buccal epithelial cell DNA methylation [31]. It is still unknown whether light-to-moderate prenatal alcohol exposure is associated with differential DNA methylation in human offspring.

In this study, we meta-analyzed epigenome-wide association study (EWAS) summary statistics from six population-based cohort studies within the Pregnancy and Childhood Epigenetics (PACE) Consortium to investigate DNA methylation profiles in the cord blood of newborns differentially exposed to alcohol in utero. We also compared these associations with those recently found in studies of differential buccal cell DNA methylation in children with FASD compared with controls [31], and differential whole blood DNA methylation in adults in the general population who drink light-to-moderately compared with adults who do not drink [32].

\section{Materials \& methods}

\section{Participating cohorts}

A total of six independent cohorts from four countries participated in this study, all were members of the PACE Consortium. Detailed methods for each cohort are provided in the Supplementary Material (Supplementary File 1). All cohorts had data on maternal alcohol consumption before and/or during pregnancy and DNA methylation data as measured using the Illumina Infinium HumanMethylation450k BeadChip array [33]. In alphabetical order, 
these cohorts were: The Avon Longitudinal Study of Parents and Children (ALSPAC) [34-37] from the UK, Groningen Expert Center for Kids with Obesity (GECKO) [38] and Generation R [39,40] from The Netherlands, two independent datasets from the Norwegian Mother and Child Cohort Study (MoBa1, MoBa2) [41,42] and Project Viva (Viva) from the USA [43].

\section{Maternal alcohol consumption (exposure)}

Cohorts assessed maternal alcohol consumption before and during pregnancy via questionnaires completed by the mothers during pregnancy. We were primarily interested in the effects of sustained consumption throughout pregnancy, which represents a longer prenatal exposure to alcohol, potentially interfering with all stages of embryonic and fetal development. Therefore, our main exposure of interest was a binary variable comparing offspring of mothers who drank both before pregnancy and in the second and/or third trimester of pregnancy to offspring of mothers who consumed alcohol before pregnancy but not during the second and/or third trimester of pregnancy. Using this definition, we hoped to compare offspring of mothers who continued to drink alcohol after finding out they were pregnant to offspring of mothers who stopped drinking. From previous research we know that women who drink in the second trimester tend to do so at light-to-moderate levels [6].

Cohorts ran secondary models assessing binge drinking during pregnancy and timing-specific alcohol consumption: before pregnancy, during the first trimester and during the second and/or third trimester. Binge drinking was defined as four, five or six (depending on the cohort) or more glasses per occasion at least once at any time point in pregnancy compared with consuming alcohol before pregnancy and drinking in moderation (i.e., no binge drinking) during pregnancy. Alcohol consumption before pregnancy, during the first trimester and during the second and/or third trimester were all defined using four categories of exposure: no drinking, less than one glass per week, one to six glasses per week and seven or more glasses per week.

\section{Covariates}

All models were adjusted for the potential confounders maternal age (years), maternal education (variable defined by each individual cohort) and maternal smoking status (the preferred categorization was into three groups: no smoking in pregnancy, stopped smoking in early pregnancy, smoking throughout pregnancy. A binary categorization of any versus no smoking was also acceptable). All cohorts also adjusted for technical covariates either by including a batch variable (e.g., chip ID) as a model covariate or by generating and adjusting for surrogate variables. All models were run with and without adjustment for cell counts, which were estimated using the Houseman method [43]. The analyzes were completed before a cord blood reference panel was widely available, so cohorts used an adult whole blood reference [44] to estimate the proportion of B cells, CD8 ${ }^{+} \mathrm{T}$ cells, $\mathrm{CD} 4^{+} \mathrm{T}$ cells, granulocytes, NK cells and monocytes in each sample.

\section{Methylation measurements (outcome)}

DNA from cord blood underwent bisulfite conversion using the EZ-96 DNA methylation kit (Zymo Research Corporation, CA, USA). DNA methylation was measured using the Illumina Infinium HumanMethylation $450 \mathrm{k}$ BeadChip assay at Illumina or in cohort-specific laboratories. Each cohort conducted its own quality control and normalization of methylation data, as detailed in the Supplementary Material (Supplementary File 1). In all analyzes, cohorts used normalized, untransformed $\beta$-values. As a consortium, we have found that extreme outliers in methylation data, likely caused by technical error or rare genetic variants, can have a large influence on results. Therefore, potential outliers were removed. Such outliers were defined using the Tukey method [45], in which an outlier is any value less than the lower quartile minus three-times the interquartile range, or more than the upper quartile plus three-times the interquartile range. This method is appropriate as it is not dependent on distributional assumptions of the data.

\section{Cohort-specific statistical analysis}

Each cohort performed independent EWAS according to a common, prespecified analysis plan. Full EWAS were conducted for each of the alcohol exposures (i.e., sustained alcohol consumption, binge drinking, drinking before pregnancy, drinking in the first trimester, drinking in the second/third trimester). Models were run using multiple robust linear regression ( $\mathrm{rlm}$ in the MASS R package [46]) in an attempt to control for potential heteroscedasticity in the methylation data. Alcohol consumption was modelled as the exposure and cord blood DNA methylation was the outcome, with adjustment for covariates (and estimated cell counts). 


\section{Meta-analysis}

We performed fixed-effects meta-analysis weighted by the inverse of the variance with METAL [47]. We then excluded control probes $(n=65)$ and probes mapped to the $X(n=11,232)$ or $Y(n=416)$ chromosomes. This left a total of 473,864 probes. Multiple testing was accounted for by controlling the false discovery rate (FDR) at 5\% using the method by Benjamini and Hochberg [48]. Probes were annotated according to hg19 using the IlluminaHumanMethylation450kanno.ilmn12.hg19 R package [49]. We used these annotations to assess enrichment in certain genomic features (relation to $\mathrm{CpG}$ island and genomic region) using Fisher's tests.

\section{Sensitivity analyzes}

For the top 500 sites with the smallest p-values in our main model (sustained drinking), we repeated the meta-analysis using a random effects model, to allow for potential differences in effect sizes between cohorts. We additionally assessed interstudy heterogeneity and influence of individual cohorts by observing forest plots and heterogeneity statistics, as well as conducting a 'leave-one-out' analysis using the metafor R package [50]. We checked consistency between models by comparing effect estimates and top hits to those of our primary model. We also compared top hits to a list of probes suggested to give spurious readings due to cross-hybridization or genomic features such as nearby SNPs [51]. When a cord blood reference became available [52], we ran a sensitivity analysis in ALSPAC adjusting for estimated proportions of B-cells, $\mathrm{CD}^{+} \mathrm{T}$ cells, $\mathrm{CD} 4^{+} \mathrm{T}$ cells, granulocytes, NK-cells, monocytes and nucleated red blood cells.

\section{Comparison to associations between FASD \& buccal epithelial DNA methylation in children} We performed a look-up in our results of the top CpGs and differentially methylated regions (DMRs; FDR-adjusted p-value $<0.05$, n CpGs $=658$ ) reported in a study by Portales-Casamar et al. [31], which analyzed epigenome-wide buccal epithelial cell DNA methylation in children with FASD compared with controls. We compared direction of effect and p-values across the two studies.

\section{Comparison to associations between light-to-moderate drinking \& whole blood DNA methylation in adults}

We performed a second look-up in our results of the top CpGs (FDR-adjusted p-value < 0.05) from a study by Liu et al. [32], which meta-analyzed associations between alcohol consumption and epigenome-wide whole blood DNA methylation in adults (CHARGE consortium). In order to harmonize with our models, we restricted the look-up to the top CpGs from the previous study's models that assessed light drinking (three CpGs) and moderate drinking (24 CpGs), versus no drinking (results supplied by the study authors). As with our first look-up, we compared direction of effect and p-values across the two studies.

\section{DMRs analysis}

Adjacent probes on the $450 \mathrm{k}$ array are often highly correlated and DMRs may be more biologically important than individual CpGs. However, there is currently no agreed 'gold-standard' method to identify DMRs and the currently available methods test slightly different (but not necessarily competing) hypotheses. Therefore, to provide confidence that any findings are robust to DMR method, we used two methods, Comb-P [53] and DMRcate [54], to identify DMRs in our meta-analyzed single-CpG EWAS results. Comb-P identifies genomic regions enriched for low p-values, corrects for auto-correlation with neighboring CpGs within 1000 bp using the Stouffer-Liptak method, then adjusts for multiple testing using the Sidak correction. DMRcate generates two smoothed estimates

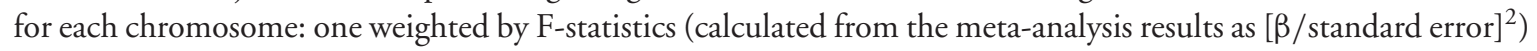
and one not, for null comparison. The two estimates are compared via a Satterthwaite approximation and $\mathrm{p}$-values are calculated and adjusted for multiple testing using the FDR method. Regions are defined from groups of significant probes $($ FDR $<0.05)$ where the distance to the next consecutive probe is less than $1000 \mathrm{bp}$. A regional $\mathrm{p}$-value is calculated using the Stouffer method. As a sensitivity analysis, we repeated the Comb-P and DMRcate analyzes using a 500 bp (rather than 1000 bp) window to define neighboring CpGs.

\section{Blood/brain comparison}

To assess whether identified associations between prenatal alcohol exposure and cord blood methylation are likely to represent associations in a more biologically relevant tissue (the brain), we performed a look-up of CpG sites in a database of correlations between blood and brain methylation [55]. Methods used to derive this database are 


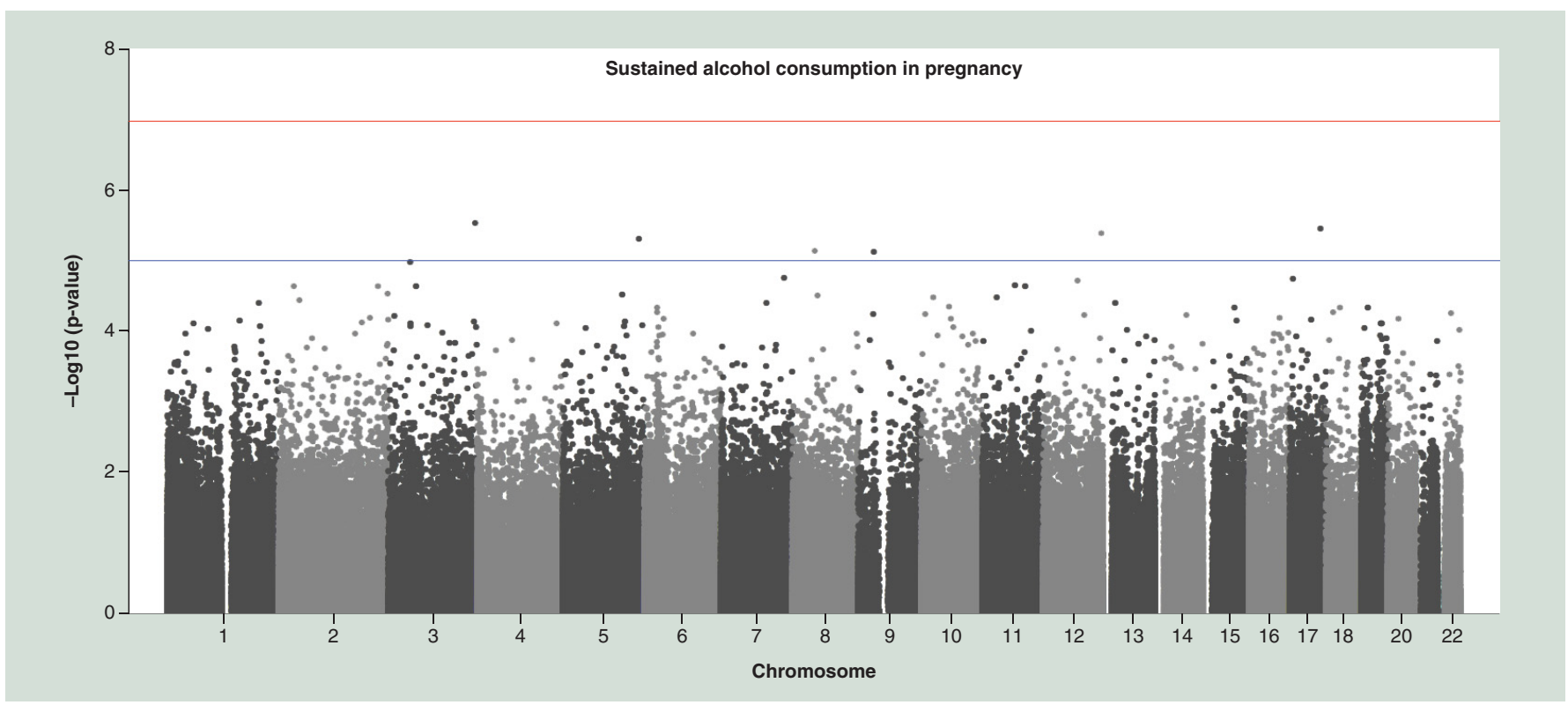

Figure 1. Manhattan plot of sustained alcohol consumption (without adjustment for cell counts).

described elsewhere [56], but briefly, the authors quantified DNA methylation in matched DNA samples from whole blood and four brain regions (prefrontal cortex, entorhinal cortex, superior temporal gyrus and cerebellum) from 122 individuals.

\section{Availability of data}

Data supporting the results reported in this article can be found in the Supplementary Materials. We regret that we are unable to make individual level data available due to concerns regarding compromising individual privacy. However, full meta-analysis results are available from the corresponding author on request.

\section{Results}

\section{Study characteristics}

Of the six participating cohorts, Project Viva and GECKO could not take part in all meta-analyzes due to insufficient data/number of exposed individuals. Project Viva was therefore only included in the analysis of drinking before pregnancy compared with abstaining. GECKO was included in the primary analysis assessing sustained alcohol consumption, and the secondary analysis assessing binge drinking. The other cohorts (ALSPAC, Generation R, MoBa1 and MoBa2) were included in all analyzes.

Five cohorts (ALSPAC, GECKO, Generation R, MoBa1, MoBa2) had the necessary data to take part in our primary analysis of sustained alcohol consumption in pregnancy. The meta-analysis included 3075 mother-child pairs, of which 1147 (37.3\%) mothers consumed alcohol both before and throughout pregnancy and the remaining 1928 mothers consumed alcohol before pregnancy/during the first trimester but not during the second and/or third trimester. Table 1 summarizes the characteristics of each cohort. In all investigated cohorts, women who drank throughout pregnancy were, on average, older and had a higher level of education than women who stopped. In $\mathrm{MoBa} 2$ and GECKO women who drank throughout pregnancy were less likely to smoke throughout pregnancy compared with women who stopped drinking, but the opposite was true for the other three studies.

\section{Primary models: sustained maternal alcohol consumption during pregnancy}

For both the cell-adjusted and cell-unadjusted models, effect sizes were moderate: for top CpGs with p-value $<1$ $\times 10^{-3}$, estimates ranged from a $4 \%$ decrease to $2 \%$ increase in average methylation level in the exposed compared with the unexposed group, with a median absolute estimate of $0.4 \%$ (Supplementary File 2, Supplementary Tables $1 \& 2$ ). No CpG sites survived correction for multiple testing with an FDR-adjusted p-value $<0.05$ (Figure 1). There was little evidence of inflation, as assessed by the lambda value (Table 1). Results (including effect sizes) for all sites with a p-value $<1 \times 10^{-3}$ are presented in Supplementary File 2, Supplementary Tables $1 \& 2$. 
Research Article Sharp, Arathimos, Reese et al.

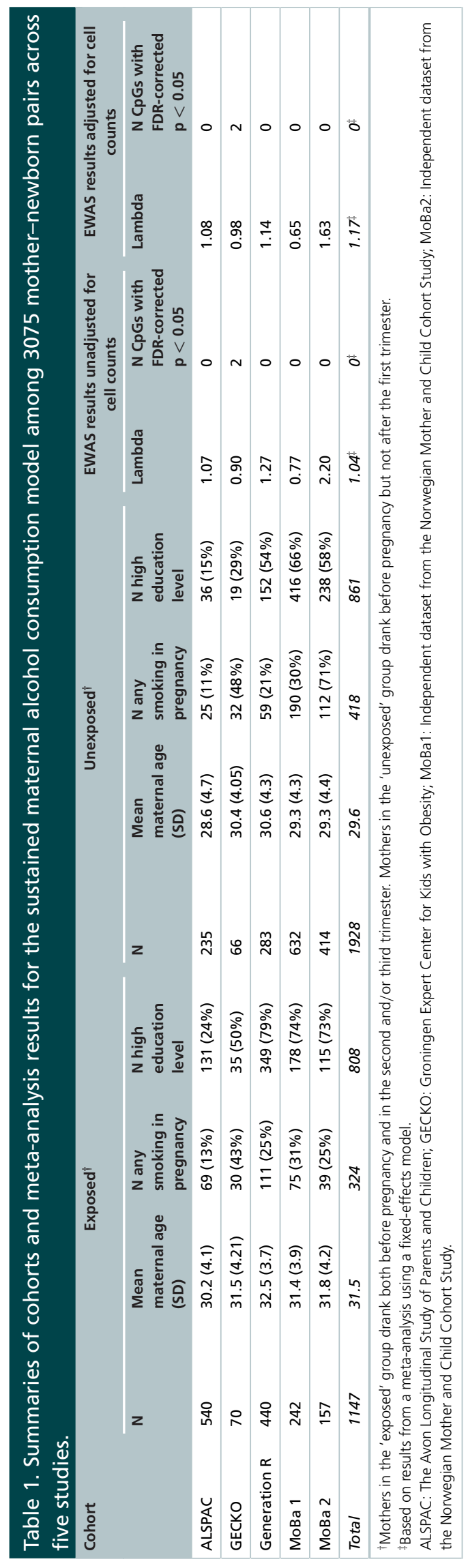


Of the 622 and $797 \mathrm{CpGs}$ with p-value $<1 \times 10^{-3}$ in the cell-unadjusted and cell-adjusted models, respectively, 500 had a p-value $<1 \times 10^{-3}$ according to both models. Adjusting for estimated cell counts appeared to have little effect: at the 500 top sites, the median percentage difference in effect sizes before and after adjustment was $3.4 \%$ and only $26 / 500$ sites changed by $10 \%$ or more. At exactly half of the 500 top sites, adjusting for cell counts reduced the effect size toward the null. At the remaining 250 sites the effect size increased after cell-adjustment. Adjusting for cell counts increased the standard error at 357/500 sites.

The top $500 \mathrm{CpGs}$ (with a p-value $<1 \times 10^{-3}$ according to both the cell-adjusted and cell-unadjusted models) were enriched at $\mathrm{CpG}$ islands ( $42.4 \%$ of the top 500 vs $30.9 \%$ in the whole array; $\mathrm{p}<0.0001)$ and first exons $(7.2$ vs $4.7 \% ; \mathrm{p}=0.0075)$, but under-represented in the open sea $(22.2$ vs $36.2 \% ; \mathrm{p}<0.0001)$ or gene bodies $(25.2$ vs $33.3 \% ; \mathrm{p}=0.0001$.

\section{Secondary models}

With and without adjustment for cell counts, no individual $\mathrm{CpG}$ sites were associated with drinking before pregnancy or during the first trimester after FDR correction for multiple testing. One CpG (cg12509712 near $A R S G)$ was associated with binge drinking, but only after adjustment for cell counts. In addition, one CpG (cg20334115 near PYCR2) was associated with drinking in the second and/or third trimester, but only before adjustment for cell counts. We did not consider these individual sites further due to the lack of consistency between the cell-adjusted and cell-unadjusted models. Results (including effect sizes) for all sites with a p-value $<1 \times 10^{-3}$ are presented in Supplementary File 2, Supplementary Tables 3-10.

\section{Sensitivity analyzes}

There was evidence of heterogeneity at a minority of the top 500 sites associated with sustained maternal alcohol consumption: 100/500 sites had a heterogeneity $\mathrm{p}$-value $<0.05$; $\mathrm{I}^{2}$ was $>40$ at $36 / 500$. After running a random effects meta-analysis at the top 500 , the coefficients for $88 / 500$ changed $>10 \%$ compared with coefficients generated using the fixed effects meta-analysis. Forest plots and results of a leave-one-out analysis (Supplementary File 3) suggested that no single cohort had a disproportionately large influence on the meta-analysis results consistently over all 500 sites.

Of the top 500 sites, 164 were on a published list of possibly problematic probes [51] (Supplementary File 2, Supplementary Tables $1 \& 2$ ). Although these sites may be more likely to contain outliers, cohorts removed outlier values prior to EWAS and used a regression model that is designed to be robust to outliers in the outcome variable.

The direction of effect in our primary model (sustained maternal alcohol consumption during pregnancy) was mostly consistent with results from our other models (Figure 2). As is expected given their similarity, results from the model assessing maternal alcohol consumption in the second/third trimester were particularly consistent with results from the primary model (Spearman's correlation coefficient for regression coefficients at all probes: 0.76 for the cell-adjusted models and 0.74 for the cell-unadjusted models). Results for all sites with a p-value $<1 \times 10^{-3}$ are presented in Supplementary File 2, Supplementary Tables 1-10 and lambdas and number of hits per cohort are provided in Supplementary File 2, Supplementary Table 11.

In ALSPAC, adjusting for cell counts estimated using the adult whole blood reference panel provided similar EWAS results to those obtained when adjusting for cell counts estimated using a cord blood reference panel: at the top 500 sites from the primary meta-analysis, the median percentage change in coefficients in ALSPAC was 7\% (IQR 3-14\%), with 317/500 sites changing less than $10 \%$. Of the top 500 sites, 219 had a crude p-value $<0.05$ in ALSPAC when using the adult reference and 197/219 (90\%) also had a crude p-value $<0.05$ when using the cord blood reference.

\section{Comparison to associations between FASD \& buccal epithelial DNA methylation in children}

Of 658 CpG sites at which DNA methylation in buccal cells was associated with FASD according to PortalesCasamar et al., 288 (44\%) had the same direction of association (regardless of p-value) in our study of maternal sustained drinking, but none survived correction for multiple testing at 658 sites (FDR-adjusted p-value $<0.05$ ). Of 542 CpG sites within 101 FASD-associated DMRs (identified using DMRcate), $215(40 \%)$ had the same direction of association in our study of maternal sustained drinking, but none survived correction for multiple testing at 542 sites (FDR-adjusted p-value $<0.05$ ). For all CpGs, the estimated effect sizes reported by Portales-Casamar et al. were larger than those found in our study. Full look-up results for all PACE models are provided in Supplementary File 2, Supplementary Table 12. 


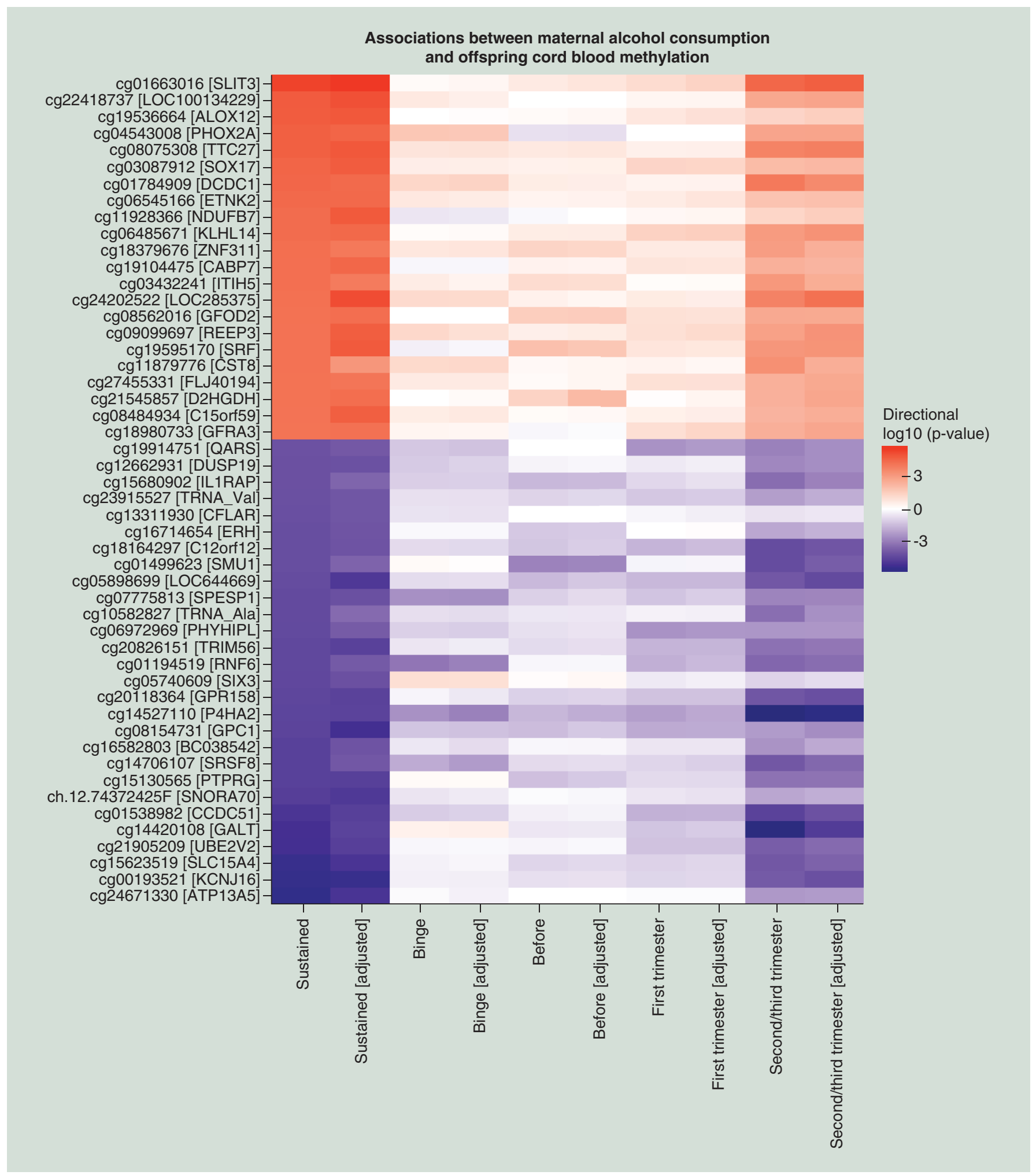

Figure 2. A heatmap to illustrate the direction and strength of association between all investigated alcohol exposures and offspring cord blood DNA methylation. Plotted CpGs are the top $50 \mathrm{CpGs}$ with the smallest p-values in the sustained alcohol consumption single-site EWAS (without adjustment for estimated cell proportions). "Adjusted" denotes models that were adjusted for estimated cell proportions. 


\begin{tabular}{|c|c|c|c|c|}
\hline Differentially-methylated region (DMR) & CpGs in DMR & Closest gene & $\begin{array}{l}\text { Sidak p-value (unadjusted } \\
\text { for cell counts) }\end{array}$ & $\begin{array}{l}\text { Sidak p-value (adjusted } \\
\text { for cell counts) }\end{array}$ \\
\hline Chr1: 152161237-152162026 & 7 & HRNR & $1.0 \times 10^{-13}$ & $6.0 \times 10^{-11}$ \\
\hline Chr16: 1583810-1584517 & 8 & IFT140 & $8.7 \times 10^{-13}$ & $5.9 \times 10^{-10}$ \\
\hline Chr6: 49681178-49681775 & 9 & CRISP2 & $9.5 \times 10^{-11}$ & $7.6 \times 10^{-08}$ \\
\hline Chr5:110062343-110062838 & 7 & TMEM232 & $2.1 \times 10^{-10}$ & $2.0 \times 10^{-07}$ \\
\hline Chr17: 6899085-6899759 & 12 & ALOX12 & $1.3 \times 10^{-09}$ & $9.3 \times 10^{-07}$ \\
\hline Chr12: 31271783-31272120 & 4 & DKFZp434C0631 & $8.8 \times 10^{-09}$ & $1.2 \times 10^{-05}$ \\
\hline Chr19: 57741988-57742445 & 10 & AURKC & $1.2 \times 10^{-08}$ & $1.2 \times 10^{-05}$ \\
\hline Chr8: $43131260-43131657$ & 5 & POTEA & $3.8 \times 10^{-07}$ & $4.5 \times 10^{-04}$ \\
\hline Chr5: 8457548-8458090 & 6 & LOC100505738 & $5.5 \times 10^{-07}$ & $4.8 \times 10^{-04}$ \\
\hline Chr13: 76334583-76334867 & 4 & LMO7 & $5.5 \times 10^{-07}$ & $4.8 \times 10^{-04}$ \\
\hline Chr5: 42953543-42953625 & 3 & AK056817 & $9.4 \times 10^{-07}$ & $1.6 \times 10^{-03}$ \\
\hline Chr2: 118594304-118594650 & 3 & $D D \times 18$ & $1.2 \times 10^{-06}$ & $7.1 \times 10^{-03}$ \\
\hline Chr3: 145879277-145879711 & 7 & PLOD2 & $1.5 \times 10^{-06}$ & $2.1 \times 10^{-03}$ \\
\hline Chr17: 35423405-35423817 & 4 & AATF & $2.4 \times 10^{-06}$ & $2.6 \times 10^{-03}$ \\
\hline Chr18: 77659572-77659696 & 2 & KCNG2 & $4.1 \times 10^{-06}$ & $4.7 \times 10^{-03}$ \\
\hline Chr15: 69222988-69223369 & 3 & SPESP1 & $4.6 \times 10^{-06}$ & $1.7 \times 10^{-02}$ \\
\hline Chr2: 103236861-103237269 & 2 & SLC9A2 & $6.1 \times 10^{-06}$ & $7.6 \times 10^{-03}$ \\
\hline Chr6: 31846769-31847029 & 10 & SLC44A4 & $6.4 \times 10^{-06}$ & $7.4 \times 10^{-03}$ \\
\hline Chr4: 3516534-3516759 & 4 & LRPAP1 & $9.2 \times 10^{-06}$ & $1.7 \times 10^{-02}$ \\
\hline
\end{tabular}

Comparison to associations between light/moderate drinking \& whole blood DNA methylation in adults

Of 24 CpGs associated (FDR-adjusted $\mathrm{p}<0.05$ ) with adult moderate drinking in the study by Liu et al. [32], one was associated with maternal sustained drinking in our PACE analysis after correction for multiple testing at 24 sites (FDR-adjusted $\mathrm{p}<0.05$ ). At this site, the direction of the effect of maternal drinking on methylation was inverse compared with the effect of own drinking: cg19909613; closest gene TTC35; PACE effect 0.0075 p-value $1.7 \times 10^{-3}$; Liu et al. effect -0.014 p-value $9.2 \times 10^{-7}$. In the cell-adjusted model, this site did not survive FDR correction. None of the three CpG sites that were associated with light drinking according to Liu et al. were associated with maternal sustained drinking in the PACE study. For all CpGs, the estimated effect sizes reported by Liu et al. were larger than those found in our study. Full look-up results for all PACE models are provided in Supplementary File 2, Supplementary Table 13.

DMR analysis

Using Comb-P to conduct a region-based analysis based on spatial correlation of p-values, we identified 30 and 32 DMRs in the cell-unadjusted and cell-adjusted maternal sustained drinking models, respectively (Sidak-corrected p-value $<0.05$ ). Nineteen regions were differentially methylated according to both models (Table 2; results for all models shown in Supplementary File 2, Supplementary Table 14). However, we found no DMRs when we conducted the region-based analysis using DMRcate. Defining DMRs using a 500 bp (as opposed to $1000 \mathrm{bp}$ ) window did not change our results using either Comb-P or DMRcate.

\section{Blood/brain comparison}

CpGs within the 19 sustained drinking DMRs identified using Comb-P tended to show strong correlations between blood and brain methylation. For example, at the top CpG with the smallest EWAS p-value within the top DMR with the smallest SIDAK-corrected p-value (cg26320663, DMR Chr1:152161237-152162026 an intergenic region on a $\mathrm{CpG}$ island near $H R N R$ ) correlation coefficients between blood and brain ranged from 0.61 in the cerebellum and entorhhinal cortex to 0.66 in the prefrontal cortex (p-values ranging $1.8 \times 10^{-8}$ to $1.5 \times$ $\left.10^{-10}\right)$. All results are presented in Supplementary File 2, Supplementary Table 15. 


\section{Discussion}

We combined data across six pregnancy cohorts to evaluate associations between maternal alcohol consumption during pregnancy and genome-wide DNA methylation in cord blood of offspring. Although we did not find any consistent evidence of association between any category of prenatal alcohol exposure, defined in terms of dose and timing, and single-site methylation, we found some evidence that 19 larger regions of the genome were differentially methylated in association with sustained maternal alcohol consumption throughout pregnancy (i.e., when comparing mothers who drank throughout pregnancy to those who stopped at the beginning or after the first trimester). However, optimal methods for regional analyzes are currently a matter of debate and we were not able to validate this finding using a different region-based method, so we conclude that we have not found any strong evidence for an association.

There are three potential interpretations of the results of our study: light-to-moderate prenatal alcohol exposure does not affect DNA methylation, any association between light-to-moderate prenatal alcohol exposure and DNA methylation is not detectable in cord blood (but could occur in other relevant tissues, e.g. the brain) and a causal association exists, but low statistical power, heterogeneity, measurement error (e.g., targeting the wrong CpG sites), confounding and bias (individually or in combination) prevented us from finding evidence of an association between prenatal alcohol exposure and cord blood DNA methylation.

Interpretation one: no effect of light-to-moderate prenatal alcohol exposure on DNA methylation The first interpretation, that prenatal alcohol exposure does not affect DNA methylation, seems at odds with a body of research in animals that suggests an effect [25-29]. However, it is important to consider alcohol dosage when comparing these studies to our own. Previous population-based studies have shown that women who drink during pregnancy tend to do so at light-to-moderate levels, especially those that drink after pregnancy detection [6]. The dose of prenatal alcohol exposure in our study is likely to be less than that considered by many animal studies. As outlined in the Introduction, evidence of fetal harm caused by light-to-moderate alcohol consumption during pregnancy is inconsistent [7-18]. Therefore, it could be argued that we would not expect light-to-moderate prenatal alcohol exposure to be strongly associated with variation in DNA methylation, either due to low power to detect very small associations, or because there is no causal effect.

Differences in the range of alcohol exposure could also partially explain why we found very limited overlap between our findings and those of a study by Portales-Casamar et al., comparing buccal cells from a small sample of children with and without FASD [31]. At the CpGs that Portales-Casamar et al. identified as associated with FASD, less than half showed the same direction of association with sustained maternal alcohol consumption in our study, and none survived correction for multiple testing. Additionally, the majority of FASD-associated differentially methylated CpGs were in gene bodies, whereas CpGs in gene bodies were significantly underrepresented amongst the top $500 \mathrm{CpGs}$ with the smallest meta-EWAS p-values in our study. The FASD case-control comparison likely covers a larger range of exposure (i.e., cases were exposed to much higher intensity of exposure in utero; controls were likely to have been exposed to less alcohol) than our study of differential prenatal exposure to alcohol in the general population.

However, differences in findings are also likely to arise given the stark methodological differences between the two studies: First, DNA methylation is strongly tissue specific [57], so DNA from buccal cells (Portales-Casamar et al.) and cord blood (our study) are perhaps unlikely to show the same general methylation patterns. Second, DNA methylation is strongly influenced by age [58] and could be affected by many factors in the postnatal environment that are associated with prenatal alcohol exposure (such as maternal education and childhood adversity) - both of these factors are important to consider when interpreting results from our study, where methylation was measured at birth, compared with the study by Portales-Casamar et al., where participants were around 11 years old. Thirdly, the prospective cohort design of the studies included in our meta-analysis meant that we were able to adjust for measured confounders and participants were sampled from the same populations. Portales-Casamar et al.'s casecontrol study of 11-year-olds is more open to confounding (especially by the postnatal environment, as mentioned above). In particular, the cases and controls differed with respect to important sociodemographic characteristics (namely, ethnicity and being raised by adoptive/foster parents) that have previously been associated with variation in DNA methylation [59-61]. Fourthly, the difference in level of alcohol exposure in both studies means there are different confounding structures, for example, in Portales-Casamar et al. higher exposure was associated with lower socioeconomic status, whereas, in our study, higher exposure was associated with higher maternal education in all investigated cohorts. 
We also saw limited similarities between our findings and those of a study recently published by Liu et al., which found some evidence of association between DNA methylation and varying levels of alcohol consumption in an adult population [32]. At the CpGs that were associated with light or moderate drinking in Liu et al., we found no strong evidence of an association with prenatal exposure. This lack of overlap might be explained by maternal alcohol consumption affecting own but not fetal DNA methylation. This would provide further support the hypothesis that light-to-moderate prenatal alcohol exposure is not associated with DNA methylation. However, other plausible explanations are that maternal alcohol consumption affects fetal DNA methylation at different CpG sites compared with adult methylation, and/or the lack of overlap is related to differences in population age, definitions, range and duration of exposure, and potential differences in accuracy of self-report in nonpregnant and pregnant adults leading to differences in measurement error.

Interpretation two: the effect of prenatal alcohol consumption on DNA methylation is not detectable in cord blood

The second possible interpretation of our results is that any association between prenatal alcohol exposure and DNA methylation is not detectable in cord blood. Although we did not find strong evidence of an association in offspring cord blood, this does not exclude the possibility that such an association does exist in a different tissue. For example, brain tissue may be a more appropriate tissue to study because some of the strongest evidence from observational studies suggests an association between prenatal alcohol exposure and impaired neurodevelopmental outcomes [62]. There are obvious ethical issues that preclude collection of brain tissue in population-based studies, however, there are reasons to believe that DNA methylation in blood may be a good surrogate for DNA methylation in brain at some sites. Strong correlations in blood and brain DNA methylation have been found at some CpGs [56,63], including at our top DMR from the Comb-P analysis. Although we were not able to replicate our Comb-P DMRs using a different method (DMRcate), the high correlation between some of these regions in blood and brain mean they may serve as interesting candidate genes for further studies of the role of DNA methylation as a mediator of associations between prenatal alcohol exposure and offspring neurodevelopmental outcomes. Alternatively, the high correlations between blood and brain methylation at these sites might represent a genetic influence on methylation that is also associated with maternal alcohol consumption. Regardless of causality, methylation at these regions might be a useful biomarker for prenatal alcohol exposure [64], thereby serving as a more objective measure than self-report. This possibility would have to be validated and tested in independent datasets.

Interpretation three: the effect of prenatal alcohol consumption on DNA methylation is not detectable due to methodological limitations

Our study has many strengths, including the use of data from six well-characterized and established cohorts. The prospective data available from these cohorts has allowed us to investigate the timing and strength of exposure, including binge-drinking and exposure by trimester, and to minimize measurement error and recall bias. Previous studies from the PACE consortium have used a similar methodology to prescribe cohort-specific analyzes and to meta-analyze results from several cohorts. These studies identified many, seemingly robust, associations [22,65-66]. This suggests that the lack of associations identified in our study is not likely due to poor EWAS or meta-analysis methodology or data. However, the third possible interpretation of our findings is that methodological elements such as statistical power, heterogeneity, confounding and bias prevented us from finding strong evidence of an association between maternal alcohol consumption during pregnancy and offspring cord blood DNA methylation.

Failure to observe associations between maternal alcohol consumption and cord blood DNA methylation could be due to lack of statistical power, particularly if we hypothesize a dose-response association such that low levels of exposure correspond to small methylation differences. As most pregnant women in our cohorts did not drink excessive amounts of alcohol, a large sample size would be required to detect a small epigenetic effect.

A further potential limitation of our study is that there was some intercohort heterogeneity. Sources of this heterogeneity include differences in the range of alcohol exposure, for example, in our main analysis, ALSPAC, GECKO and Generation R have more exposed than unexposed individuals, while the MoBa cohorts have a higher proportion of unexposed individuals. These differences are perhaps expected, because public health advice on drinking during pregnancy has changed over time in different countries. Other potential sources of heterogeneity include differences in how alcohol drinking is measured, confounding structures (we note that in some studies women who drank throughout pregnancy were more likely to be smokers, while in others the opposite was true) and/or EWAS methodological differences such as different methods of normalizing DNA methylation data, coding 
covariates and adjusting for batch. Encouragingly though, our meta-analysis results were not substantially different when we used a random-effects model compared with a fixed-effects model. Furthermore, forest plots and results of a leave-one-out analysis suggested our meta-analysis results were not strongly influenced by differences between studies. In a previous PACE analysis [22], we found that results obtained using raw $\beta$ s were similar to those obtained using $\beta$ s normalized by various methods, which indicates that the method of normalization did not impact the inference drawn from the meta-analysis. Furthermore, nonspurious associations would likely be robust to small differences in methodology between studies.

Measurement error could also reduce statistical power and this may be a particular problem for studies of maternal alcohol consumption: pregnant women may under-report behaviors that are widely thought to be harmful for their baby [67]. Similarly, classification bias may introduce measurement error, for example, harmonizing the definition of binge drinking was particularly difficult because binge drinking was defined differently in different cohorts. As a result, women consuming four drinks on one occasion would be classed as binge drinkers in some cohorts but nonbinge drinkers in others. This reduces the power to detect a true effect.

Cellular heterogeneity in cord blood samples is a further issue [68] that may introduce error in our measure of DNA methylation. Blood samples are highly heterogeneous and although we have attempted to adjust for cellular heterogeneity by including estimated cell proportions in our EWAS models, no suitable cord blood reference was widely available at the time of analysis, so these estimates were based on an adult blood reference panel. When a cord blood reference became available [52], a sensitivity analysis in ALSPAC did not reveal sizable differences between EWAS results adjusted for cell proportions estimated using either the cord or adult reference panel. However, we recognize that there may be a residual influence of cellular heterogeneity that could be biasing the results in either direction. Another factor that could be biasing results is circulating folate levels, which we could not formally evaluate because not all cohorts had the required data. However, there are two reasons why folate is likely to play at most a marginal role in our findings. First, we found low inter-study heterogeneity for most of our CpG sites - if there was a prominent interaction between alcohol (a folate antagonist) and folate (a methyl donor), then we might expect more heterogeneity due to country- and timing-specific differences in folate intake. Secondly, our results were largely null - confounding by folic acid supplementation, which is indirectly associated with alcohol consumption [14,69], is unlikely to be exaggerating estimates of the association between maternal alcohol and offspring methylation.

We also consider that some of our findings may be affected by selection bias: women who are actively trying to get pregnant may abstain from drinking alcohol, but these women were excluded from our analyzes of sustained and binge drinking. However, these women were not excluded from our analyzes of other alcohol exposures (before pregnancy, first trimester, second and third trimester) and our findings were consistently null across all alcohol exposures.

A final potential limitation is that the Illumina HumanMethylation $450 \mathrm{k}$ array covers only $1.7 \%$ of all CpG sites in the human genome, which may not cover potential regions where maternal alcohol consumption is most strongly associated with offspring methylation. In particular, the array is biased toward regulatory regions, whereas the majority of prenatal alcohol-associated regions identified by Portales-Casamar et al. were in intergenic regions or gene bodies [31]. However, it should be noted that Portales-Casamar et al. also used the Illumina HumanMethylation450k array, so limitations in array coverage are unlikely to explain differences in findings between these studies. The development of sequence-based approaches methods with better genomic coverage will help overcome this limitation in future studies.

If an association between maternal alcohol consumption and cord blood DNA methylation does exist (e.g., in larger samples and/or different populations with a greater range of alcohol exposure), then future studies should explore whether that association is causal or explained by some of the issues that plague observational studies, such as those discussed above. Confounding by genetics or shared mother-child environmental factors is a particular concern that should be addressed. For example, paternal alcohol consumption could be employed as a 'negative control' that will share the same confounding structure as maternal alcohol consumption but cannot plausibly affect offspring DNA methylation through a direct causal intrauterine mechanism [70]. Techniques such as two-step Mendelian randomization [71] could also be applied to explore the causal effect of prenatal alcohol exposure on DNA methylation and the causal effect of DNA methylation on offspring outcomes. Even if the association is not causal, newborn blood DNA methylation might capture both genetic and environmental influences of maternal alcohol consumption, which could be useful, both clinically and in research, as a biomarker of exposure and/or a useful predictor of offspring outcomes. 


\section{Conclusion}

In this multicohort study, we found no evidence that maternal alcohol consumption during pregnancy is associated with offspring cord blood DNA methylation in the general population. However, it is possible that exploration of a combination of different tissues, higher doses and different timings of exposure, as well as a more global assessment of genomic DNA methylation, might show evidence of association. We therefore recommend caution when interpreting the present null findings and encourage further investigations.

Summary points

- It is well known that heavy alcohol consumption during pregnancy can cause Fetal Alcohol Spectrum Disorders (FASD), but evidence is lacking or mixed on the effects of light-to-moderate drinking.

- Whether there is a causal association between light-to-moderate drinking in pregnancy and children's health outcomes is an important question, and identifying a possible biological pathway showing effects at birth would be a step toward providing an answer.

- Some evidence from animal studies suggests that epigenetic mechanisms, such as DNA methylation, might mediate associations between prenatal alcohol exposure and health outcomes.

- In the Pregnancy and Childhood Epigenetics (PACE) consortium, we meta-analyzed associations between maternal drinking in pregnancy and genome-wide cord blood DNA methylation across six population-based birth cohorts, looking at sustained drinking throughout pregnancy, binge drinking and time-specific exposures.

- Our main analysis of sustained maternal drinking included 3075 mother-child pairs, of which 1147 (37.3\%) mothers consumed alcohol both before and throughout pregnancy and the remaining 1928 mothers consumed alcohol before pregnancy/during the first trimester but not during the second and/or third trimester (i.e., they stopped drinking after pregnancy detection).

- We found no strong evidence of association between prenatal alcohol exposure and cord blood DNA methylation at either individual $\mathrm{CpGs}$ or across larger regions of the genome.

- We consider three potential interpretations of the results of our study: light-to-moderate prenatal alcohol exposure does not affect DNA methylation, any association between light-to-moderate prenatal alcohol exposure and DNA methylation is not detectable in cord blood (but could occur in other relevant tissues, e.g., the brain) and a causal association is there, but low statistical power, heterogeneity, measurement error (e.g., targeting the wrong CpG sites), confounding and bias (individually or in combination) prevented us from finding evidence of an association between prenatal alcohol exposure and cord blood DNA methylation.

- It is possible that exploration of a combination of different tissues, higher doses and different timings of exposure, as well as a more global assessment of genomic DNA methylation, might show evidence of association between maternal alcohol consumption and cord blood DNA methylation in the general population.

- We recommend caution when interpreting our null findings and encourage further investigations.

Financial \& competing interests disclosure

The ALSPAC cohort was supported by the Wellcome Trust (WT088806). The Project Viva cohort is funded by NIH grants R01 HL111108, R01 NR013945, and R01 HD034568. The authors have no other relevant affiliations or financial involvement with any organization or entity with a financial interest in or financial conflict with the subject matter or materials discussed in the manuscript apart from those disclosed.

No writing assistance was utilized in the production of this manuscript.

Ethical conduct of research

The authors state that they have obtained appropriate institutional review board approval or have followed the principles outlined in the Declaration of Helsinki for all human or animal experimental investigations. In addition, for investigations involving human subjects, informed consent has been obtained from the participants involved.

Open access

This article is distributed under the terms ofthe Creative Commons Attribution License 4.0 which permits any use, distribution, and reproduction in any medium, provided the original author(s) and the source are credited. To view a copy of the license, visit http://creativecommons.org/licenses/by/4.0/ 
Supplementary data

To view the supplementary data that accompany this paper please visit the journal website at: www.futuremedicine.com/doi/full/10.2217/epi-2017-0095

\section{References}

Papers of special note have been highlighted as: $\bullet$ of interest; $\bullet \bullet$ of considerable interest

1. Jones KL, Smith DW. Recognition of the fetal alcohol syndrome in early infancy. Lancet 302(7836), 999-1001 (1973).

2. Abel EL. Fetal Alcohol Abuse Syndrome. Springer Science and Business Media, NY, USA (1998).

3. Sokol RJ, Delaney-Black V, Nordstrom B. Fetal alcohol spectrum disorder. JAMA 290(22), 2996-2999 (2003).

4. Bonthius DJ, Goodlett CR, West JR. Blood alcohol concentration and severity of microencephaly in neonatal rats depend on the pattern of alcohol administration. Alcohol 5(3), 209-214 (1988).

5. Goodlett CR, Horn KH, Zhou FC. Alcohol teratogenesis: mechanisms of damage and strategies for intervention. Exp. Biol. Med. (Maywood) 230(6), 394-406 (2005).

6. O'Keeffe LM, Kearney PM, McCarthy FP et al. Prevalence and predictors of alcohol use during pregnancy: findings from international multicentre cohort studies. BMJ Open 5(7), e006323 (2015).

- Provides interesting and much-needed evidence on the prevalence of alcohol use during pregnancy. It shows that, in the general population, most women who drink during pregnancy do so at light-to-moderate levels.

7. Henderson J, Gray R, Brocklehurst P. Systematic review of effects of low-moderate prenatal alcohol exposure on pregnancy outcome. BJOG An. Int. J. Obstet. Gynaecol. 114(3), 243-252 (2007).

8. McCarthy FP, O'Keeffe LM, Khashan AS et al. Association between maternal alcohol consumption in early pregnancy and pregnancy outcomes. Obstet. Gynecol. 122(4), 830-837 (2013).

9. Patra J, Bakker R, Irving H, Jaddoe V, Malini S, Rehm J. Dose-response relationship between alcohol consumption before and during pregnancy and the risks of low birthweight, preterm birth and small for gestational age (SGA)-a systematic review and meta-analyses. BJOG An. Int. J. Obstet. Gynaecol. 118(12), 1411-1421 (2011).

10. O'Keeffe LM, Greene RA, Kearney PM. The effect of moderate gestational alcohol consumption during pregnancy on speech and language outcomes in children: a systematic review. Syst. Rev. 3(1), 1 (2014).

11. Gaskins AJ, Rich-Edwards JW, Williams PL, Toth TL, Missmer SA, Chavarro JE. Prepregnancy low to moderate alcohol intake is not associated with risk of spontaneous abortion or stillbirth. J. Nutr. 146(4), 799-805 (2016).

12. Flak AL, Su S, Bertrand J, Denny CH, Kesmodel US, Cogswell ME. The association of mild, moderate, and binge prenatal alcohol exposure and child neuropsychological outcomes: a meta-analysis. Alcohol. Clin. Exp. Res. 38(1), 214-226 (2014).

13. Bay B, Kesmodel US. Prenatal alcohol exposure - a systematic review of the effects on child motor function. Acta Obstet. Gynecol. Scand. 90(3), 210-226 (2011).

14. Zuccolo L, DeRoo LA, Wills AK et al. Pre-conception and prenatal alcohol exposure from mothers and fathers drinking and head circumference: results from the Norwegian Mother-Child Study (MoBa). Sci. Rep. 7, 39535 (2016).

15. Mamluk L, Edwards HB, Savović J et al. Low alcohol consumption and pregnancy and childhood outcomes: time to change guidelines indicating apparently 'safe' levels of alcohol during pregnancy? A systematic review and meta-analyses. BMJ Open 7(7), e015410 (2017).

- This is a comprehensive review of all the available studies of light alcohol consumption and pregnancy and childhood outcomes since the 1950s. It finds surprisingly limited evidence that light drinking during pregnancy poses any risk to the baby, but there was a weak association with preterm delivery and being small-for-gestational-age.

16. Lewis SJ, Zuccolo L, Davey Smith G et al. Fetal alcohol exposure and IQ at age 8: evidence from a population-based birth-cohort study. PLoS ONE 7(11), e49407 (2012).

17. Zuccolo L, Lewis SJ, Davey Smith G et al. Prenatal alcohol exposure and offspring cognition and school performance. A 'Mendelian randomization' natural experiment. Int. J. Epidemiol. 42(5), 1358-1370 (2013).

18. Murray J, Burgess S, Zuccolo L, Hickman M, Gray R, Lewis SJ. Moderate alcohol drinking in pregnancy increases risk for children's persistent conduct problems: Causal effects in a Mendelian randomisation study. J. Child Psychol. Psychiatry Allied Discip. 57(5), 575-584 (2016).

19. Gray R. Low-to-moderate alcohol consumption during pregnancy and child development - moving beyond observational studies. BJOG 120(9), 1039-1041 (2013).

20. Niclasen J. Drinking or not drinking in pregnancy: the multiplicity of confounding influences. Alcohol Alcohol. 49(3), 349-355 (2014).

21. Department of Health. UK Chief Medical Officers' Alcohol Guidelines Review: summary of the proposed new guidelines (2016). www.gov.uk/government/uploads/system/uploads/attachment_data/file/489795/summary.pdf

22. Joubert BR, Felix JF, Yousefi P et al. DNA methylation in newborns and maternal smoking in pregnancy: Genome-wide Consortium meta-analysis. Am. J. Hum. Genet. 98(4), 680-696 (2016). 
-. This previous study from the PACE consortium identified many strong associations between maternal smoking throughout pregnancy and DNA methylation in offspring cord blood at birth and peripheral blood later in childhood.

23. Richmond RC, Simpkin AJ, Woodward G et al. Prenatal exposure to maternal smoking and offspring DNA methylation across the lifecourse: findings from the Avon Longitudinal Study of Parents and Children (ALSPAC). Hum. Mol. Genet. 24(8), 2201-2217 (2015).

24. Küpers LK, Xu X, Jankipersadsing SA et al. DNA methylation mediates the effect of maternal smoking during pregnancy on birthweight of the offspring. Int. J. Epidemiol. 44(4), 1224-1237 (2015).

25. Halsted $\mathrm{CH}$, Medici V. Aberrant hepatic methionine metabolism and gene methylation in the pathogenesis and treatment of alcoholic steatohepatitis. Int. J. Hepatol. 2012, 959746 (2012).

26. Hutson JR, Stade B, Lehotay DC, Collier CP, Kapur BM. Folic acid transport to the human fetus is decreased in pregnancies with chronic alcohol exposure. PLoS ONE 7(5), e38057 (2012).

27. Stouder C, Somm E, Paoloni-Giacobino A. Prenatal exposure to ethanol: a specific effect on the H19 gene in sperm. Reprod. Toxicol. 31(4), 507-512 (2011).

28. Perkins A, Lehmann C, Lawrence RC, Kelly SJ. Alcohol exposure during development: Impact on the epigenome. Int. J. Dev Neurosci. 31(6), 391-397 (2013).

29. Laufer BI, Chater-Diehl EJ, Kapalanga J, Singh SM. Long-term alterations to DNA methylation as a biomarker of prenatal alcohol exposure: From mouse models to human children with fetal alcohol spectrum disorders. Alcohol 60, 67-75 (2017)

30. Khalid O, Kim JJ, Kim H-S et al. Gene expression signatures affected by alcohol-induced DNA methylomic deregulation in human embryonic stem cells. Stem Cell Res. 12(3), 791-806 (2014).

31. Portales-Casamar E, Lussier AA, Jones MJ et al. DNA methylation signature of human fetal alcohol spectrum disorder. Epigenetics Chromatin. 9, 25 (2016).

-. This is one of the only previously published studies of prenatal alcohol exposure and DNA methylation in humans. The authors found several regions differentially methylated in buccal cells from children with and without FASD. In the current paper, we discuss the many possible reasons for very limited overlap between these results and our own.

32. Liu C, Marioni RE, Hedman AK et al. A DNA methylation biomarker of alcohol consumption. Mol. Psychiatry. doi:10.1038/mp.2016.192 (2016) (Epub ahead of print).

-. This meta-analysis from the CHARGE + consortium identified many CPG sites where DNA methylation is associated with alcohol consumption in adults. We performed a look-up of their results within our dataset and found very limited overlap. Potential reasons are discussed in the current paper.

33. Sandoval J, Heyn HA, Moran S et al. Validation of a DNA methylation microarray for 450,000 CpG sites in the human genome. Epigenetics 6(6), 692-702 (2011).

34. Relton CL, Gaunt T, McArdle W et al. Data Resource Profile: Accessible Resource for Integrated Epigenomic Studies (ARIES). Int. J. Epidemiol. 44(4), 1181-1190 (2015).

35. Boyd A, Golding J, Macleod J et al. Cohort Profile: the 'children of the $90 \mathrm{~s}$ ' - the index offspring of the Avon Longitudinal Study of Parents and Children. Int. J. Epidemiol. 42(1), 111-127 (2013).

36. Fraser A, Macdonald-Wallis C, Tilling K et al. Cohort Profile: the Avon Longitudinal Study of Parents and Children: ALSPAC mothers cohort. Int. J. Epidemiol. 42(1), 97-110 (2013).

37. L'Abee C, Sauer PJ, Damen M, Rake J-P, Cats H, Stolk RP. Cohort Profile: the GECKO Drenthe study, overweight programming during early childhood. Int. J. Epidemiol. 37(3), 486-489 (2008).

38. Kruithof CJ, Kooijman MN, van Duijn CM et al. The Generation R Study: Biobank update 2015. Eur. J. Epidemiol. 29(12), 911-27 (2014).

39. Kooijman MN, Kruithof CJ, van Duijn CM et al. The Generation R Study: design and cohort update 2017. Eur. J. Epidemiol. 31(12), 1243-1264 (2016).

40. Magnus P, Irgens LM, Haug K et al. Cohort profile: the Norwegian Mother and Child Cohort Study (MoBa). Int. J. Epidemiol. 35(5), $1146-50$ (2006).

41. Rønningen KS, Paltiel L, Meltzer HM et al. The biobank of the Norwegian Mother and Child Cohort Study: a resource for the next 100 years. Eur. J. Epidemiol. 21(8), 619-25 (2006).

42. Oken E, Baccarelli AA, Gold DR et al. Cohort profile: project viva. Int. J. Epidemiol. 44(1), 37-48 (2015).

43. Houseman EA, Accomando WP, Koestler DC et al. DNA methylation arrays as surrogate measures of cell mixture distribution. $B M C$ Bioinformatics 13(1), 86 (2012).

44. Reinius LE, Acevedo N, Joerink M et al. Differential DNA methylation in purified human blood cells: implications for cell lineage and studies on disease susceptibility. PLoS ONE 7(7), e41361 (2012).

45. Tukey JW. Exploratory data analysis. Addison-Wesley Publishing Company Reading, Mass. Biometric Journal 23(4), 413-414 (1981).

46. Venables W, Ripley B. Modern Applied Statistics with S. Fourth. Springer, NY, USA (2002). 
47. Willer CJ, Li Y, Abecasis GR. METAL: fast and efficient meta-analysis of genomewide association scans. Bioinformatics 26(17), 2190-2191 (2010).

48. Benjamini Y, Hochberg Y. Controlling the false discovery rate: a practical and powerful approach to multiple testing. J. R. Stat. Soc. Ser B. 57(1), 289-300 (1995).

49. Hansen K. IlluminaHumanMethylation450kanno.ilmn12.hg19: annotation for Illumina’s 450k methylation arrays. Bioconductor doi:10.18129/B9.bioc.IlluminaHumanMethylation450kanno.ilmn12.hg19 (2016) (Epub ahead of print).

50. Viechtbauer W. Conducting meta-analyses in R with the metafor Package. J. Stat. Softw. 36(3), 1-48 (2010).

51. Naeem H, Wong $\mathrm{N}$, Chatterton $\mathrm{Z}$ et al. Reducing the risk of false discovery enabling identification of biologically significant genome-wide methylation status using the HumanMethylation 450 array. BMC Genomics 15(1), 51 (2014).

52. Andrews S, Bakulski K. FlowSorted.CordBlood.450k: Illumina 450k data on sorted cord blood cells. Bioconductor doi:10.18129/B9.bioc.FlowSorted.CordBlood.450k (2016) (Epub ahead of print).

53. Pedersen BS, Schwartz DA, Yang IV, Kechris KJ. Comb-p: software for combining, analyzing, grouping and correcting spatially correlated P-values. Bioinformatics 28(22), 2986-2988 (2012).

54. Peters TJ, Buckley MJ, Statham AL et al. De novo identification of differentially methylated regions in the human genome. Epigenetics Chromatin 8(1), 6 (2015).

55. Complex Disease Epigenetics Group. Blood Brain DNA Methylation Comparison Tool. http://epigenetics.iop.kcl.ac.uk/bloodbrain/

56. Hannon E, Lunnon K, Schalkwyk L, Mill J. Interindividual methylomic variation across blood, cortex, and cerebellum: implications for epigenetic studies of neurological and neuropsychiatric phenotypes. Epigenetics 10(11), 1024-1032 (2015).

57. Lokk K, Modhukur V, Rajashekar B et al. DNA methylome profiling of human tissues identifies global and tissue-specific methylation patterns. Genome Biol. 15(4), r54 (2014).

58. Horvath S. DNA methylation age of human tissues and cell types. Genome Biol. 14(10), R115 (2013).

59. Fraser HB, Lam LL, Neumann SM, Kobor MS. Population-specificity of human DNA methylation. Genome Biol. 13(2), R8 (2012).

60. Essex MJ, Boyce WT, Hertzman C et al. Epigenetic vestiges of early developmental adversity: childhood stress exposure and DNA methylation in adolescence. Child Dev. 84(1), 58-75 (2013).

61. Roth TL, Lubin FD, Funk AJ, Sweatt JD. Lasting epigenetic influence of early-life adversity on the bdnf gene. Biol. Psychiatry 65(9), 760-769 (2009).

62. Birney E, Davey Smith G, Greally JM. Epigenome-wide association studies and the interpretation of disease -omics. PLoS Genet. 12(6), e1006105 (2016).

63. Walton E, Hass J, Liu J et al. Correspondence of DNA methylation between blood and brain tissue and its application to schizophrenia research. Schizophr. Bull. 42(2), 406-414 (2016).

64. Relton CL, Hartwig FP, Davey Smith G. From stem cells to the law courts: DNA methylation, the forensic epigenome and the possibility of a biosocial archive. Int. J. Epidemiol. 44(4), 1083-1093 (2015).

65. Gruzieva $\mathrm{O}, \mathrm{Xu} \mathrm{C}-\mathrm{J}$, Breton CV et al. Epigenome-wide meta-analysis of methylation in children related to prenatal $\mathrm{NO} 2$ air pollution exposure. Environ. Health Perspect. 125(1), 104-110 (2017).

66. Sharp GC, Salas LA, Monnereau C et al. Maternal BMI at the start of pregnancy and offspring epigenome-wide DNA methylation: findings from the pregnancy and childhood epigenetics (PACE) consortium. Hum. Mol. Genet. 26(20), 4067-4085 (2017).

-. This previous study from the PACE consortium found weak associations between maternal body mass index at the start of pregnancy and offspring DNA methylation. After application of causal inference techniques, we found stronger evidence that the majority of these associations were better explained by shared genetic and/or environmental factors than a causal intrauterine effect.

67. Jacobson SW, Jacobson JL, Sokol RJ, Martier SS, Ager JW, Kaplan MG. Maternal recall of alcohol, cocaine, and marijuana use during pregnancy. Neurotoxicol. Teratol. 13(5), 535-540 (1991).

68. Jaffe AE, Irizarry RA. Accounting for cellular heterogeneity is critical in epigenome-wide association studies. Genome Biol. 15(2), R31 (2014).

69. Cueto HT, Riis AH, Hatch EE, Wise LA, Rothman KJ, Mikkelsen EM. Predictors of preconceptional folic acid or multivitamin supplement use: a cross-sectional study of Danish pregnancy planners. Clin. Epidemiol. 4, 259-265 (2012).

70. Richmond RC, Al-Amin A, Davey Smith G, Relton CL. Approaches for drawing causal inferences from epidemiological birth cohorts: a review. Early Hum. Dev. 90(11), 769-780 (2014).

71. Relton CL, Davey Smith G. Two-step epigenetic Mendelian randomization: a strategy for establishing the causal role of epigenetic processes in pathways to disease. Int. J. Epidemiol. 41(1), 161-176 (2012). 


\section{Regulation of UGT1A expression by miR-298 in human livers from the Han Chinese population and in human cell lines}

Pei Wang ${ }^{1}$, Ya-li Nie ${ }^{1}$, Shu-jie Wang ${ }^{2}$, Lin-lin Yang ${ }^{1}$, Wei-hong Yang ${ }^{1}$, Jiang-feng Li ${ }^{1}$, Xiao-tian $\mathrm{Li}^{2}$ \& Li-rong Zhang*,1

${ }^{1}$ Department of Pharmacology, School of Basic Medicine, Zhengzhou University, Zhengzhou, China

${ }^{2}$ Department of Pharmacology, School of Pharmacy, Zhengzhou University, Zhengzhou, China

* Author for correspondence: Tel.: +86 371 67781855; Fax: +86 371 66913510; zhanglirongzzu@126.com

Aim: This study aimed to investigate the role of miRNAs in UGT1A regulation. Materials \& methods: Based on bioinformatic prediction results, luciferase reporter assay and cell-transfection experiments were performed to study effects of miR-298 on UGT1A expression. Correlation study was conducted in human livers. Results: miR-298 overexpression reduced mRNA level of UGT1A1 and UGT1A4 in HepG2 and LS174T cells, and that of UGT1A3 and UGT1A9 in LS174T cells. miR-298 repression increased mRNA level of UGT1A4 in HepG2 and LS174T cells, and that of UGT1A1 and UGT1A3 in LS174T cells. Inverse correlations between miR-298, as well as miR-491-3p, and UGT1A3 and 1A4 mRNA levels were observed in livers. Conclusion: The study demonstrates that miR-298 and miR-491-3p downregulates UGT1A expression.

First draft submitted: 20 May 2017; Accepted for publication: 25 September 2017; Published online: 27 November 2017

Keywords: miRNA • miR-298 • post-transcriptional regulation • UDP-glucuronosyltransferase • UGT1A

UGTs are a group of Phase II metabolizing enzymes that play a critical role in the glucuronidation of endogenous substrates and a wide range of exogenous xenobiotics [1]. In humans, three UGT subfamilies (UGT1A, UGT2A and UGT2B) are primarily responsible for glucuronidation [2]. The UGT1A family comprises nine functional enzymes (1A1, 1A3-1A10) encoded by a single gene at 2q37; members of this family possess a unique first exon, with exons 2-5 in common, and a shared 3'-UTR [3]. The UGT2 family comprises ten functional enzymes, which are located on chromosome 4q13 and encoded by individual genes of six unique exons [4]. Human UGTs are expressed in a tissue-specific manner. Most UGTs, including UGT1A1, 1A3, 1A4, 1A6 and 1A9, are expressed in the liver and exhibit substantial interindividual variation in expression [5-7].

The variability in glucuronidation by the different UGT isoforms expressed in the human liver may be substantial and is considered to underlie human disorders (e.g., Crigler-Najjar syndrome) [8,9] or unpredictable drug response [10-12]. Genetic diversity, including polymorphisms, splice variants and ligand-activated transcription factors, has been reported to contribute to the interindividual variability of UGT transcription [7,13-15]. However, these factors do not explain the tissue-specific expression of $U G T 1 A[16,17]$ or the poor correlation between $U G T 1 A$ mRNA and protein levels [18,19]. Therefore, we attempted to identify the additional mechanisms involved in the regulation of $U G T 1 A$ expression.

miRNAs are endogenous noncoding RNA molecules comprising up to $2 \%$ of mammalian genomes [20]. miRNAs bind to 3'-UTRs, coding sequences or 5'-UTRs of target mRNAs to mediate post-transcriptional regulation of gene expression, leading to inhibition of translation or mRNA degradation [21,22]. miRNAs are predicted to regulate more than $60 \%$ of human gene expression [23,24], and currently, over 2500 miRNAs have been identified in humans (miRBase, Version 21.0) [20]. Recently, numerous studies have reported that miRNAs play an important role in the regulation of DMEs, with potential impacts on drug response [25-28]. Although most investigations have focused on the regulation of CYP450 enzymes by miRNAs, only one study has demonstrated the involvement of miRNAs in the regulation of UGT1A expression. Dluzen et al. reported that miR-491-3p may regulate UGT1A1 expression in both HepG2 and Huh-7 cells [29]. However, they failed to show a correlation between miR-491-3p expression 
levels and UGT1A1 mRNA levels in the human liver. A single gene may be regulated by multiple miRNAs, and it is likely that other miRNAs are involved in the regulation of UGT1A, especially UGT1A1. Studies of gene regulation in different populations have led to controversial conclusions in clinical investigations [30]. Therefore, the mechanisms underlying the regulation of UGT1A expression by miRNAs remain to be explored; in particular, the effect of miR-491-3p on UGT1A expression in the Chinese population has yet to be elucidated.

In the present study, we show that miR-298 is involved in the regulation of UGT1A expression through in silico and in vitro studies, as well as via analysis of liver tissue from Han Chinese donors. We additionally investigated the correlations between miR-298 or miR-491-3p and UGT1A expression levels in the Han Chinese population. The present findings provide novel insights into $U G T 1 A$ regulation and serve to elucidate the mechanisms underlying the remarkable interindividual variability in $U G T 1 A$ expression.

\section{Materials \& methods}

Chemicals \& reagents

The psiCHECKII plasmid and dual luciferase reporter assay system were purchased from Promega (Beijing, China). miR-298 mimic, mimic negative control, miR-298 inhibitor and inhibitor negative control were purchased from RuiBo Company (Guangzhou, China). All primers were commercially synthesized at Takara Bio (Dalian, China). Rabbit antihuman UGT1A1 monoclonal antibody and mouse antihuman GAPDH monoclonal antibody were obtained from Abcam (Cambridge, UK). Goat antirabbit secondary antibody and goat antimouse secondary antibody conjugated to hydrogen peroxidase were obtained from Proteintech (IL, USA).

Tissues \& cell culture

Liver samples were obtained from 28 Chinese Han donors at the First Affiliated Hospital of Zhengzhou University (detailed information is presented in Supplementary Tables $1 \& 2$ ). Before enrollment, all samples underwent clinical screening. Hepatitis B and C tests were negative. All the subjects did not smoke and drink. All samples were acquired following partial hepatectomy and diagnosed by two pathologists; normal tissue areas were selected for analysis in this study. The liver samples were cut into small pieces and immediately stored in liquid nitrogen. Written informed consent was obtained from participants. All donors demonstrated normal liver function. This study was approved by the Ethics Committees of the First Affiliated Hospital of Zhengzhou University. The human embryonic kidney cell line 293 (HEK293), human hepatic cellular carcinoma cell line HepG2 and human colon carcinoma cell line LS174T were purchased from the Type Culture Collection of the Chinese Academy of Sciences (Shanghai, China). HEK293, HepG2 and LS174T cells were cultured in DMEM (Hyclone, UT, USA) supplement with 10\% fetal bovine serum (BI, Kibbutz Beit Haemek, Israel) and 1\% $100 \mathrm{U} / \mathrm{ml}$ penicillin/streptomycin (Solarbio Bio, Beijing, China) at $37^{\circ} \mathrm{C}$ with $5 \% \mathrm{CO}_{2}$.

\section{Prediction of miRNA-binding sites}

The full-length 3'-UTR sequence of the human UGT1A gene was obtained from the University of California Santa Cruz Genome Browser (hg18 assembly). miRNA-binding site prediction was performed using five online software programs, namely TargetScan (www.targetscan.org/), miRanda (www.microrna.org/microrna/home.do), PITA (http://genie.weizmann.ac.il/pubs/mir07/mir07_prediction.html), microcosm Targets (www.ebi.ac.uk/enright-srv/ microcosm/htdocs/targets/v5/, formerly referred to as miRBase Targets) and PicTar (http://pictar.mdc-berlin.de/). Analyses using these programs were performed using the default parameters. Entries for UGT1A were not found in the PicTar database.

\section{Construction of reporter plasmids}

The UGT1A 3'-UTR segment (0-684 bp, from stop codon) was amplified from human genomic DNA using primers with Xbal/NotI sites (forward primer 5'-CCGCTCGAGGAAGTGGGTGGGAAATAAGGTAA$3^{\prime}$ and reverse primer 5'-AGTT GCGGCCGCGTAAACTTGCCCAGCACTTCA). The PCR product was cloned downstream of the Renilla luciferase reporter gene within the psiCHECK II vector (Promega) after digestion with XhoI and NotI. The seed deletion plasmid was created using the Mut Express II Fast Mutagenesis Kit V2 (Vazyme Biotech Co., Nanjing, China), and the forward and reverse primers were 5'-TTTTGGAGCTGGCAGATGGTTGCAATTGATCC-3' and 5'ATCTGCCAGCTCCAAAAGATAAGATTAAAACTGCCAT-3', respectively. The nucleotide sequences of the constructed plasmids were confirmed by DNA sequencing analyses. 


\begin{tabular}{|c|c|}
\hline Primer & Sequence $\left(5^{\prime}-3^{\prime}\right)$ \\
\hline UGT1A1-F ${ }^{[18]}$ & CCT TGC CTC AGA ATT CCT TC \\
\hline UGT1A3-F ${ }^{[18]}$ & GTT GAA CAA TAT GTC TTT GGT CT \\
\hline UGT1A4-F ${ }^{[18]}$ & CCT GCT GTG TTT TTT TGG AGG T \\
\hline UGT1A6-F ${ }^{[18]}$ & CAA CTG TAA GAA GAG GAA AGA C \\
\hline UGT1A9-F ${ }^{[18]}$ & GAA CAT TTA TTA TGC CAC CG \\
\hline UGT1A-R ${ }^{[18]}$ & ATT GAT CCC AAA GAG AAA ACC AC \\
\hline GAPDH-F & GCA CCG TCA AGG CTG AGA AC \\
\hline GAPDH-R & TGG TGA AGA CGC CAG TGG A \\
\hline
\end{tabular}

\section{Luciferase reporter activity assay}

Various luciferase reporter plasmids (psiCHECKII, psiCHECKII-UGT1A 3'-UTR or seed deletion plasmid) carrying the miRNA negative control or miRNA mimic were transiently transfected into HEK293 cells. Briefly, $24 \mathrm{~h}$ before transfection, the cells were seeded into 24 -well plates. After $24 \mathrm{~h}$, the reporter plasmids (100 ng) carrying the miRNA mimic or negative control mimic were transfected using Lipofectamine 2000 (Invitrogen, CA, USA). After incubation for $48 \mathrm{~h}$, cells were resuspended in the passive lysis buffer; then, luciferase activity was measured using the dual-luciferase reporter assay system (Promega). The results were expressed as the ratio of Renilla activity to firefly activity in the lysate.

\section{Quantitative real-time PCR}

Total RNA was extracted from human liver samples and cell lines using miRCURY RNA Isolation Kit (Exiqon, Vedbaek, Denmark), according to the manufacturer's protocol. cDNAs were synthesized from total RNA using the PrimeScript RT Reagent kit (Takara Bio, Beijing, China) to determine mRNA expression levels and quantified by quantitative real-time PCR (qRT-PCR) with specific primers (Table 1) using SYBR Premix EX Taq (Takara Bio, Beijing, China). GAPDH expression levels were measured as an endogenous control. For quantification of miRNA, reverse transcription and qRT-PCR were performed using miRCURY LNA Universal RT miRNA PCR system (Exiqon). U6 snRNA was measured as the endogenous control. All qRT-PCRs were performed using a 7500 FAST Real-Time PCR System (Life Technologies, CA, USA) with at least three replicates.

\section{Western blot analyses}

HepG2 and LS174T cells were transfected with $100 \mathrm{nM}$ miR-298 mimic or negative control in 6-well plates using Lipofectamine 2000 (Invitrogen) according to the manufacturer's instructions. At $48 \mathrm{~h}$ after transfection, cell protein homogenate was prepared with RIPA (Solarbio Bio) containing protease inhibitor PMSF. Relative protein concentrations of UGT1A1 were assessed by western blot using a rabbit anti-UGT1A1 antibody and normalized to the GAPDH protein concentration.

\section{Statistical analysis}

IBM SPSS 21.0 statistical software package was used for statistical analysis. Data are expressed as the mean \pm standard error of the mean of three independent experiments. Comparison of two groups was performed via an unpaired, two-tailed Student's $t$-test. Statistical significance in differences among more than two groups was determined by ANOVA and Dunnett's multiple comparisons test. Correlation analysis was performed with Spearman's rank method. $\mathrm{p}<0.05$ was considered to represent statistical significance.

\section{Results}

UGT1A1 protein levels are not associated with UGT1A1 mRNA levels in human livers

As UGT1A1 is the most abundant member of the UGT1A family in the human liver, where it is the sole enzyme responsible for the metabolism of bilirubin [1,31], we first examined the UGT1A1 mRNA and protein levels in a panel of 28 human livers by qRT-PCR and western blot, respectively. The relationship between the levels of UGT1A1 mRNA and protein was additionally investigated. As shown in Figure 1, no statistically significant correlation was observed between UGT1A1 mRNA and protein levels $(\mathrm{r}=-0.028 ; \mathrm{p}=0.885)$. This finding 


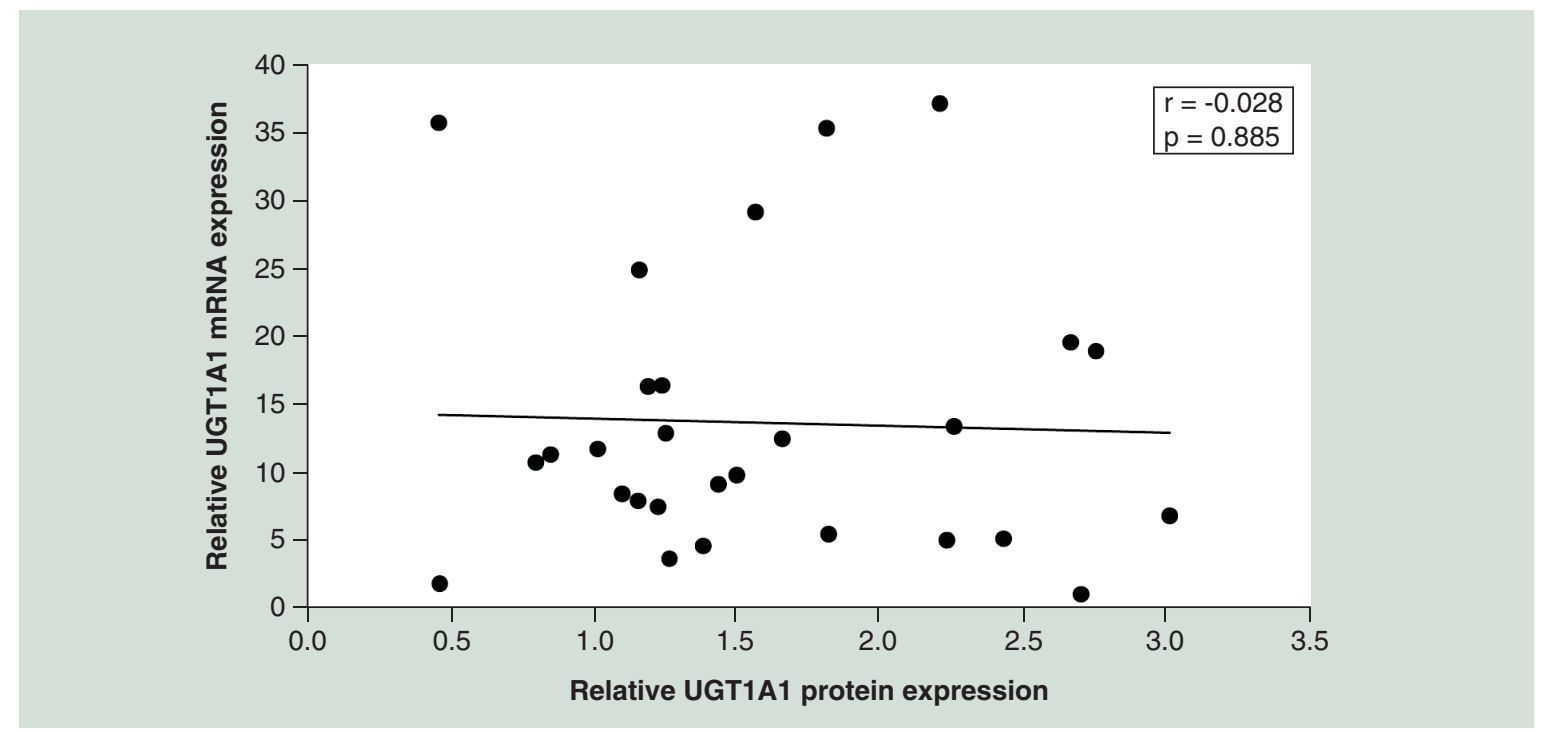

Figure 1. The relationship between UGT1A1 mRNA and protein expression levels in Chinese Han liver samples. No correlation between UGT1A1 mRNA and protein levels $(n=28)$. The UGT1A1 mRNA level was determined by qRT-PCR and normalized with the GAPDH mRNA level. The UGT1A1 protein level was determined by western blot analysis and normalized with the GAPDH protein level. Dots represent the mean \pm SEM of three independent replicates. qRT-PCR: Quantitative real-time PCR; SEM: Standard error of the mean.

\begin{tabular}{|c|c|c|c|c|c|c|c|}
\hline \multirow[t]{2}{*}{ RefSeq gene ID $^{\dagger}$} & \multirow[t]{2}{*}{$3^{\prime}$-UTR length (bp) } & \multicolumn{4}{|c|}{ Bioinformatic programs } & \multirow{2}{*}{$\begin{array}{l}\text { Total number of unique } \\
\text { miRNAs }\end{array}$} & \multirow[t]{2}{*}{ Overlap $\$$} \\
\hline & & TargetScan & miRanda & PITA & microCosm targets & & \\
\hline NM_000463.2 & 740 & 158 & 26 & 55 & 22 & 201 & 45 \\
\hline
\end{tabular}

indicates that $U G T 1 A 1$ is post-transcriptionally regulated. To elucidate the molecular mechanism underlying the post-transcriptional regulation of UGT1A1, we sought to examine whether miRNAs play a role in its regulation.

Bioinformatic analysis identifies UGT1A as a potential miRNA target

An online search using six bioinformatic algorithms identified potential recognition elements of 201 different miRNAs in the UGT1A 3'-UTR (Table 2). Among them, miR-152, miR-298, miR-548 and miR-590 exhibited high complementarity in their seed sequence and were predicted by four bioinformatic algorithms. Accordingly, in subsequent analyses in the present study we focused on these miRNAs. The alignment of the four predicted miRNAs is shown in Figure 2.

Luciferase activity of the UGT1A 3'-UTR-carrying reporter plasmid was repressed by miR-298 in vitro To determine whether the predicted miRNAs regulate UGT1A1 transcriptional activity, luciferase assays were performed using HEK293 cells (Figure 3). The selected miRNAs were cotransfected with either the psiCHECKII or the psiCHECKII-UGT1A-3'-UTR vector. Relative to the negative control miRNA, miR-298 reduced the luciferase activity of the psiCHECK2-UGT1A-3'-UTR plasmid by $29 \%$, as shown in Figure 3B. In contrast, transfection with miR-152, miR-548 and miR-590 exerted limited effects on UGT1A 3'-UTR luciferase activity. To confirm miRNA-MRE (miRNA recognition element) interactions, 4 bp of the sequence of the corresponding miR-298 MRE was deleted in the psiCHECKII-UGT1A-3'-UTR vector. Compared with UGT1A 3'-UTR-luciferase activity, the seed deletion plasmids exhibited higher luciferase activity in cells co-transfected with miR-298 (Figure 3C). These results suggest that miR-298 targets the UGT1A 3'-UTR directly, particularly at the predicted MRE sites. 


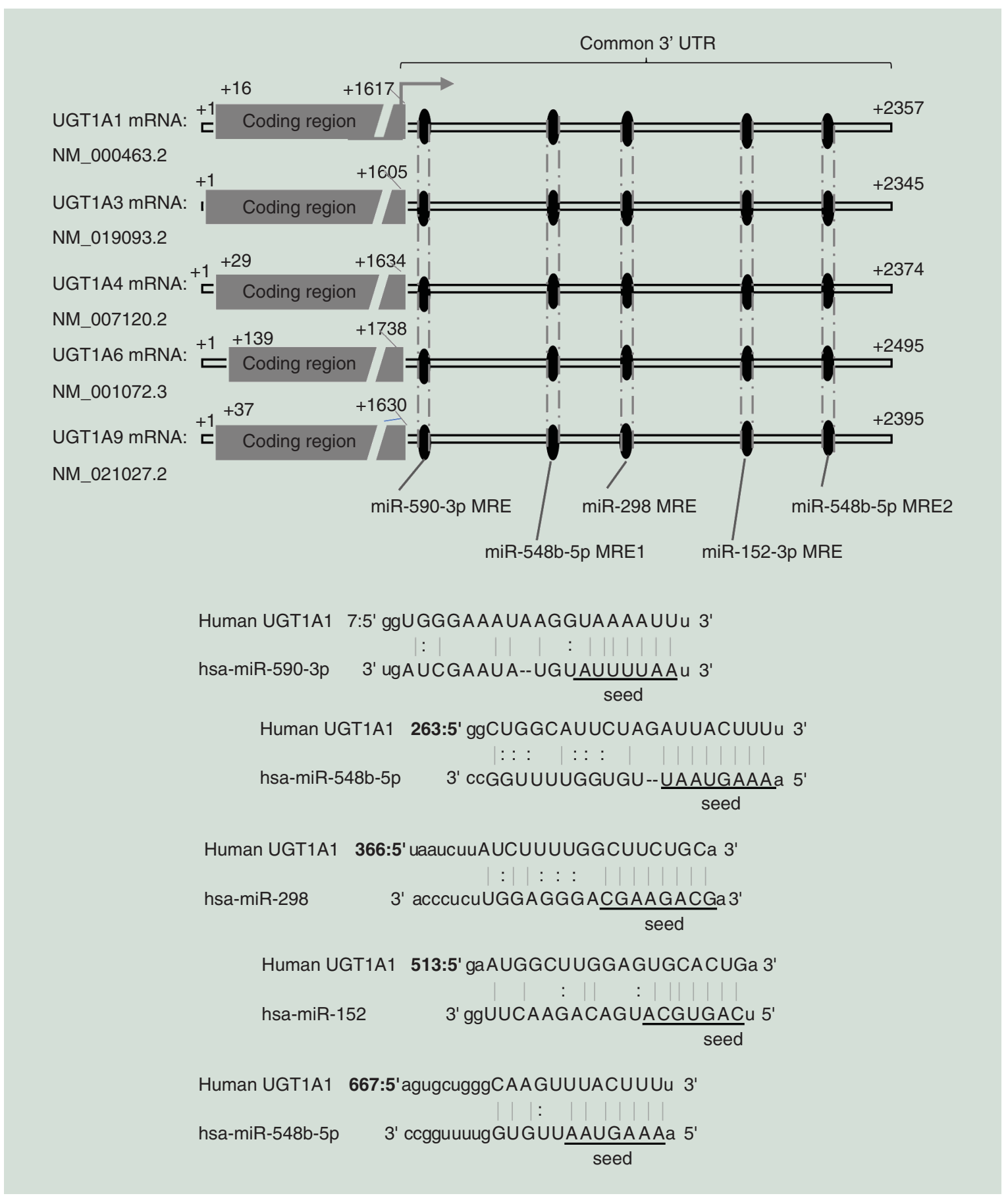

Figure 2. The potential-binding sites of predicted miRNAs in the UGT1A $3^{\prime}$ untranslated region. Schematic diagrams of human UGT1A mRNAs (UGT1A1, 1A3, 1A4, 1A6 and 1A9) and the potential-binding sites of predicted miRNAs (miR-590-3p, miR-548b-5p, miR-298 and miR-152) in the 3'-UTR are shown (upper panel). Complementarity of predicted miRNAs to UGT1A 3'-UTR is enlarged (lower panel). The miR-590-3p MRE begins +7 nucleotides from the stop codon for all UGT1A enzymes. The miR-298 and miR-152 MREs begin at +366 and +513 nucleotides, respectively. The miR-548b-5p has two MREs in UGT1A 3'-UTR, with one begins +263 and the other +667 . 


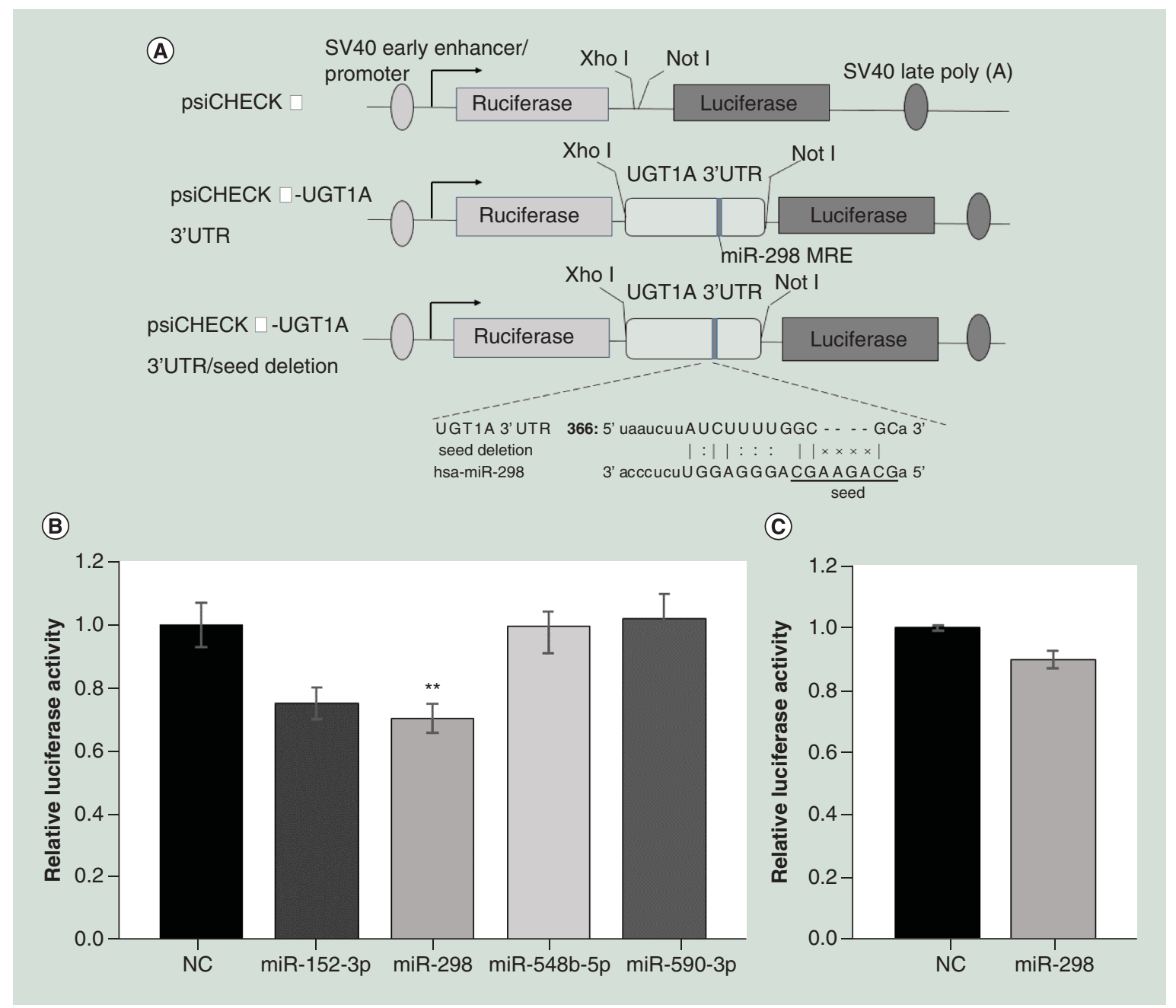

Figure 3. Analyzing the role of miRNAs in the UGT1A $3^{\prime}$-UTR with luciferase reporters. (A) Schematic representation of luciferase vectors. The psiCHECKII-UGT1A 3'-UTR vector contains the first 684 nucleotides of the UGT1A family 3'-UTR. The psiCHECKII-UGT1A 3'-UTR/seed deletion vector contains all sequences of the psiCHECKII-UGT1A 3'-UTR vector except for a four nucleotides deletion within the miR-298 MRE seed sequence. HEK293 cells were cotransfected with psiCHECKII-UGT1A 3'-UTR vector (B) or seed deletion vector (C) and miRNAs. The data shown are the ratio of the firefly luciferase activity to the Renilla luciferase activity. Columns represent the mean \pm SEM of three independent experiments in triplicates.

$* * \mathrm{p}<0.01$ compared with the NC.

NC: Negative control; SEM: Standard error of the mean.

Effects of miR-298 on UGT1A mRNA \& protein expression

To determine whether miR-298 silences the expression of hepatic UGT1A mRNA and protein, we transfected HepG2 and LS174T cells with miR-298 and the negative control miRNA. When miR-298 was transfected into HepG2 and LS174T cells, mature miR-298 levels significantly increased by 2120- and 3518-fold, respectively (Figure 4A \& C). Concomitantly, UGT1A1 and UGT1A4 mRNA levels were found to decrease in miR-298transfected HepG2 cells relative to miRNA negative control transfected cells (28 and 68\%, respectively; p $<0.05$ and $\mathrm{p}<0.01$, respectively) (Figure 4B). As observed in HepG2 cells, overexpression of miR-298 reduced the mRNA expression level of UGT1A1 and UGT1A4 in LS174T cells (43 and 69\%, respectively) (Figure 4D). In addition, UGTIA3 and UGT1A9 mRNA levels also decreased in the presence of miR-298 in LS174T cells (61 and 44\%, respectively) (Figure 4D). Consistent with the UGT1A1 mRNA levels, UGT1A1 protein levels were significantly reduced in miR-298-transfected cells compared with miRNA negative control transfected cells in HepG2 (51\%; $\mathrm{p}<0.05)$ and LS174T cells (30\%; $\mathrm{p}<0.05$ ) (Figure 5). 


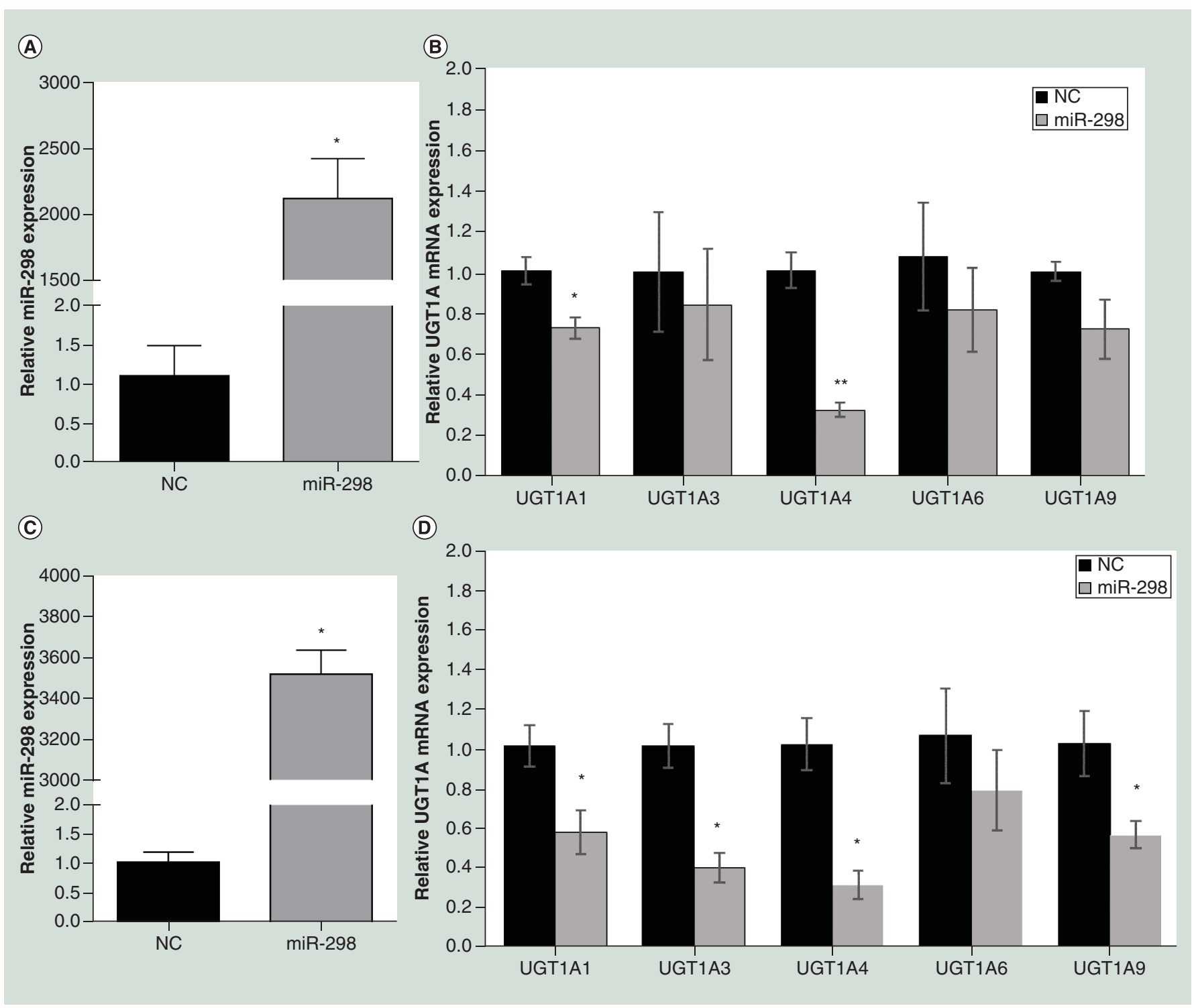

Figure 4. The effect of miR-298 overexpression on UGT1A mRNA expression in HepG2 and LS174T cells. miR-298 expression levels in miR-298 mimic or mimic negative control transfected HepG2 (A) and LS174T (C) cells; UGT1A mRNA expression levels in miR-298 mimic or mimic negative control transfected HepG2 (B) and LS174T (D) cells. The expression levels of UGT1A mRNA and miR-298 were determined by qRT-PCR and normalized to GAPDH and U6 snRNA, respectively. Columns represent the mean \pm SEM of three independent experiments in triplicates.

${ }^{*} \mathrm{p}<0.05 ;{ }^{* *} \mathrm{p}<0.01$ compared with NC.

NC: Negative control; qRT-PCR: Quantitative real-time PCR; SEM: Standard error of the mean.

To further confirm the effect of miR-298 on the expression of hepatic UGT1A, miR-298 inhibitor or inhibitor negative control and miR-298 mimic were transfected into HepG2 and LS174T cells. Briefly, at $24 \mathrm{~h}$ after transfection with the miR-298 mimic, cells were retransfected with a miR-298 inhibitor or negative control. Then, cells were harvested after $24 \mathrm{~h}$. miR-298 repression led to increase UGT1A4 mRNA expression in HepG2 cells (1.7-fold; $\mathrm{p}<0.01$; Figure 6C). In addition, the mRNA expression of UGT1A1, UGT1A3 and UGT1A4 increased in LS174T cells ( $<<0.05$; Figure 6D). Protein expression of UGT1A1 did not change in both HepG2 and LS174T cells (data not shown). 


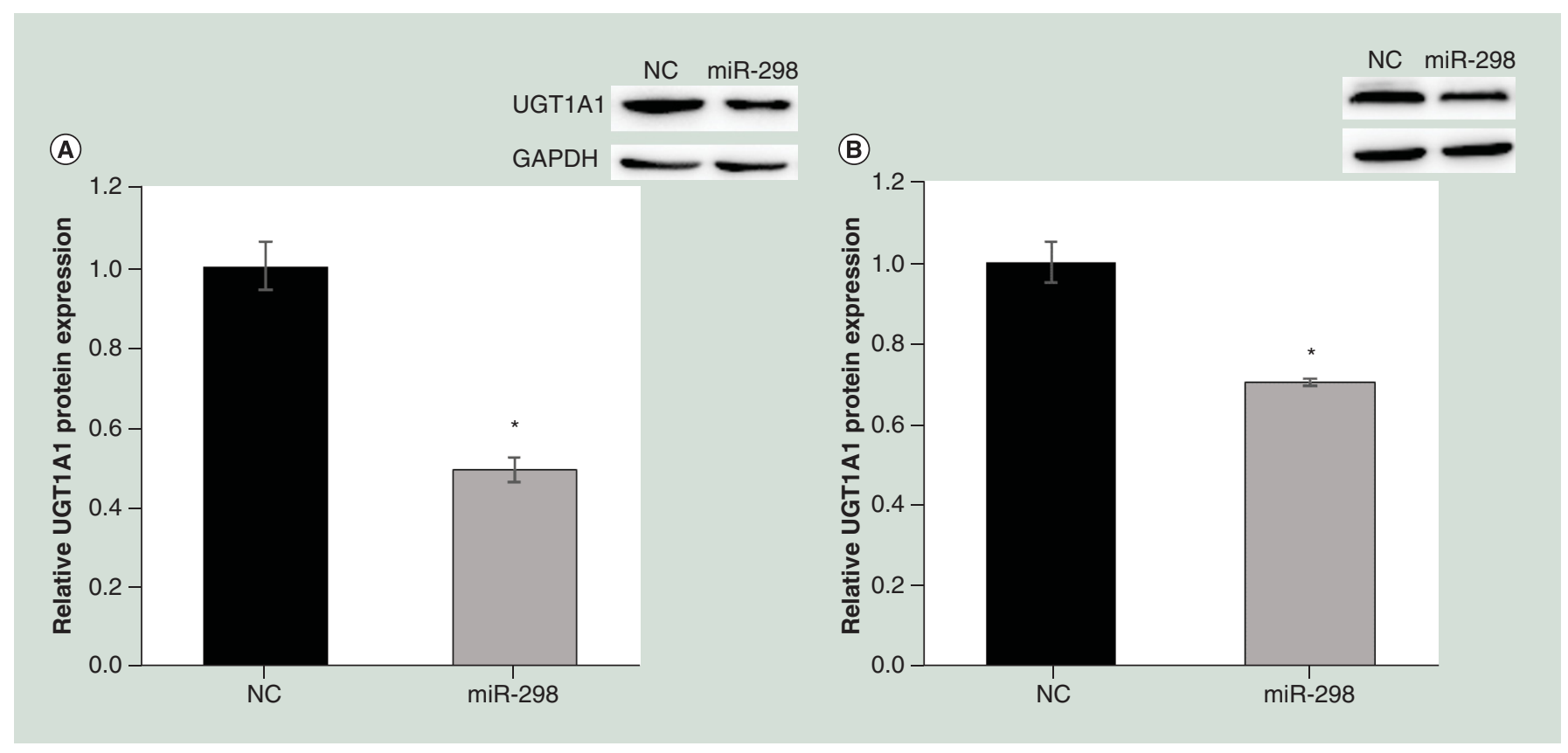

Figure 5. The effect of miR-298 overexpression on UGT1A1 protein expression in HepG2 and LS174T cells. UGT1A1 protein expression levels in miR-298 mimic or mimic negative control transfected HepG2 cells (A). UGT1A1 protein expression levels in miR-298 mimic or mimic negative control transfected LS174T cells (B). The expression level of miR-298 was determined by qRT-PCR and normalized to U6 snRNA. Quantification of UGT1A protein levels by western blot. GAPDH was used as the internal control. Columns represent the mean \pm SEM of three independent experiments in triplicates.

${ }^{*} \mathrm{p}<0.05$ compared with NC.

NC: Negative control; qRT-PCR: Quantitative real-time PCR; SEM: Standard error of the mean.

miR-298 may control UGT1A expression in human liver tissue

To investigate the effects of miR-298 expression in human liver tissue, the relationship between the expression of miR-298 and that of UGT1A was investigated using a panel of 28 human livers. As shown in Figure 7D, the miR-298 level in human livers was significantly correlated with the mRNA level of $U G T 1 A 3(\mathrm{r}=-0.404$; $\mathrm{p}<0.05)$. A similar inverse correlation was observed between miR-298 and UGT1A4 $(\mathrm{r}=-0.418 ; \mathrm{p}<0.05)$ (Figure 7E). However, there was no correlation between the mRNA level, protein level or translation efficiency of miR-298 and those of UGT1A1 (Figure 7A-C). Furthermore, no correlations were found between miR-298 and the mRNA expression of UGT1A6 or UGT1A9 ( $\mathrm{p}>0.05)$.

miR-491-3p affected the expression of UGT1A in the Chinese Han population

To assess whether miR-491-3p regulates $U G T 1 A$ expression in the Chinese Han population, we examined the relationship between miR-491-3p levels and $U G T 1 A$ levels. Significant inverse correlations were observed between miR-491-3p and UGT1A3 or UGT1A4 mRNA levels (Figure 8). However, no correlation was found between miR-491-3p and the expression levels of UGT1A1, UGT1A6 or UGT1A9.

\section{Secondary structures of UGT1A mRNA}

To investigate whether the secondary structure of $U G T 1 A$ mRNA contributes to the subtype-specific regulation mediated by miR-298 and miR-491-3p on UGT1A, the mfold Web Server (http://unafold.rna.albany.edu/?q= mfold) was used to predict the mRNA secondary structures for each hepatic UGT1A isoform. As shown in Figure 9, different $U G T 1 A$ isoforms have a unique secondary structure. In addition, the secondary structure of the miR-298-MRE in each UGT1A isoform was unique (Figure 9).

\section{Discussion}

The expression of DMEs exhibits a large degree of interindividual variability, which contributes to substantial variations in drug sensitivity, efficacy and toxicity $[1,32,33]$. Increasing evidence has indicated a role of miRNAs in the regulation of DMEs [20,26]. Although the post-transcriptional regulation of CYP450 by miRNAs is well 


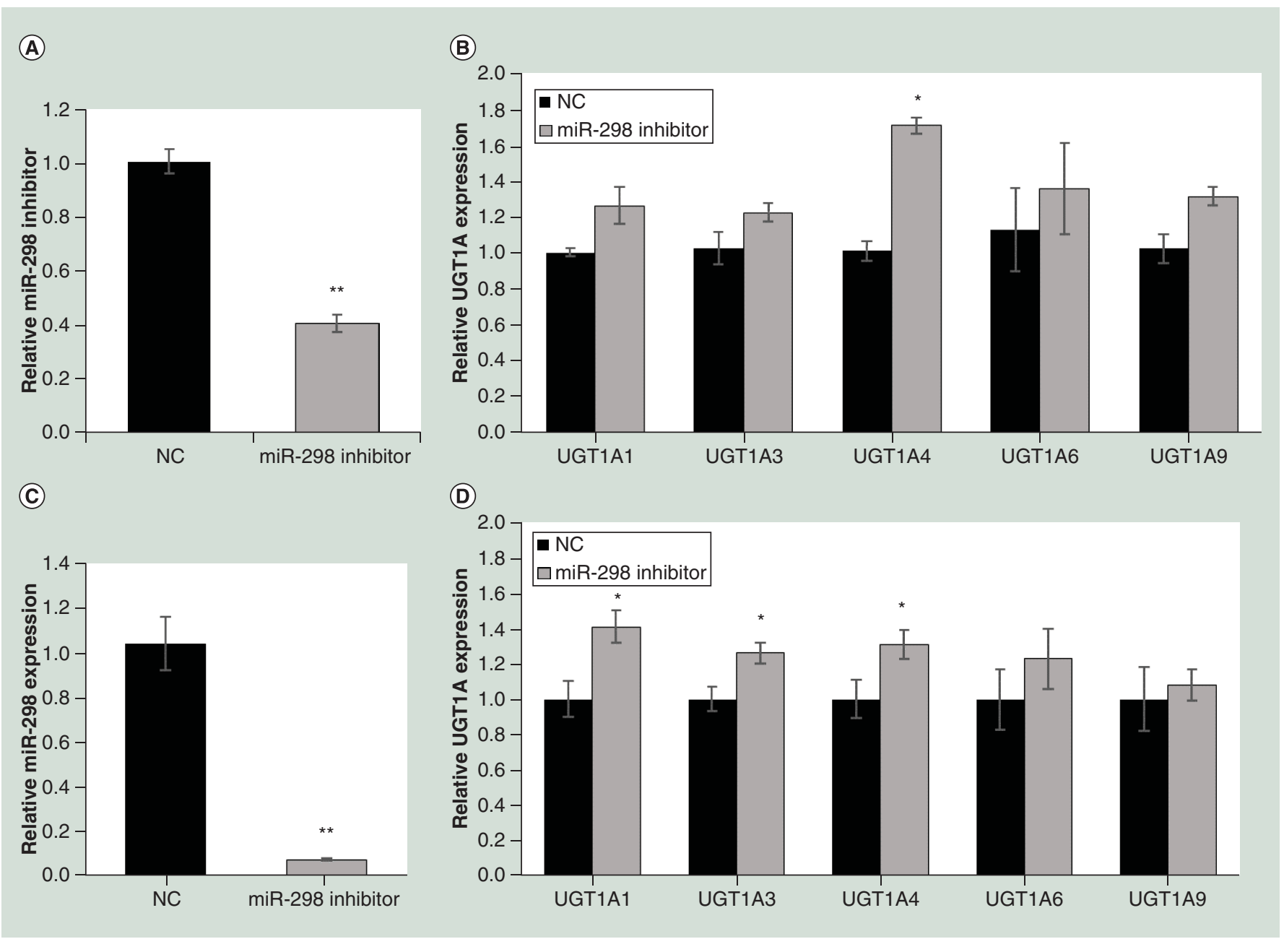

Figure 6. The effect of miR-298 downexpression on UGT1A mRNA expression in HepG2 and LS174T cells. miR-298 expression levels in miR-298 inhibitor or inhibitor negative control transfected HepG2 (A) and LS174T cells (C); UGT1A mRNA expression levels in miR-298 inhibitor or inhibitor negative control transfected HepG2 (B) and LS174T (D) cells. Columns represent the mean \pm SEM of three independent experiments in triplicates.

${ }^{*} \mathrm{p}<0.05 ;{ }^{* *} \mathrm{p}<0.01$ compared with NC.

NC: Negative control; SEM: Standard error of the mean.

understood, few reports of the impact of miRNAs on $U G T$, especially $U G T 1 A$, are available. The present study provides evidence for the role of miRNAs in the regulation of UGT1A.

We first demonstrated that there was no significant correlation between the protein and mRNA levels of UGT1A1 in the human liver samples studied; this finding was in agreement with those of previous studies [19]. The present bioinformatic analysis predicted that the UGT1A 3'-UTR is targeted by various miRNAs. These results together suggest that miRNAs are involved in the post-transcriptional regulation of $U G T 1 A 1$. As each database utilized different measurement principles [34,35], there was substantial interalgorithm variability in the miRNAs predicted to target the UGT1A 3'-UTR. On the basis of the number of algorithms predicting these miRNAs and their binding energy, hsa-miR-152, hsa-miR-298, hsa-miR-548b-5p and hsa-miR-590-3p were selected for further analysis via luciferase reporter assay. Among the four candidate miRNAs, only miR-298 was found to repress luciferase activity. Therefore, this study reports the first identification of the functional MRE for miR-298 in UGT1A 3'-UTR (Figure 3).

HepG2 and LS174T cells transfected with miR-298 mimic both exhibited significant reduction of UGT1A1 mRNA and protein expression compared with negative control cells (Figures $4 \& 5$ ). And the extents of the effects on both the mRNA and protein levels of UGT1A1 were not consistent. It is likely that miR-298 regulates 


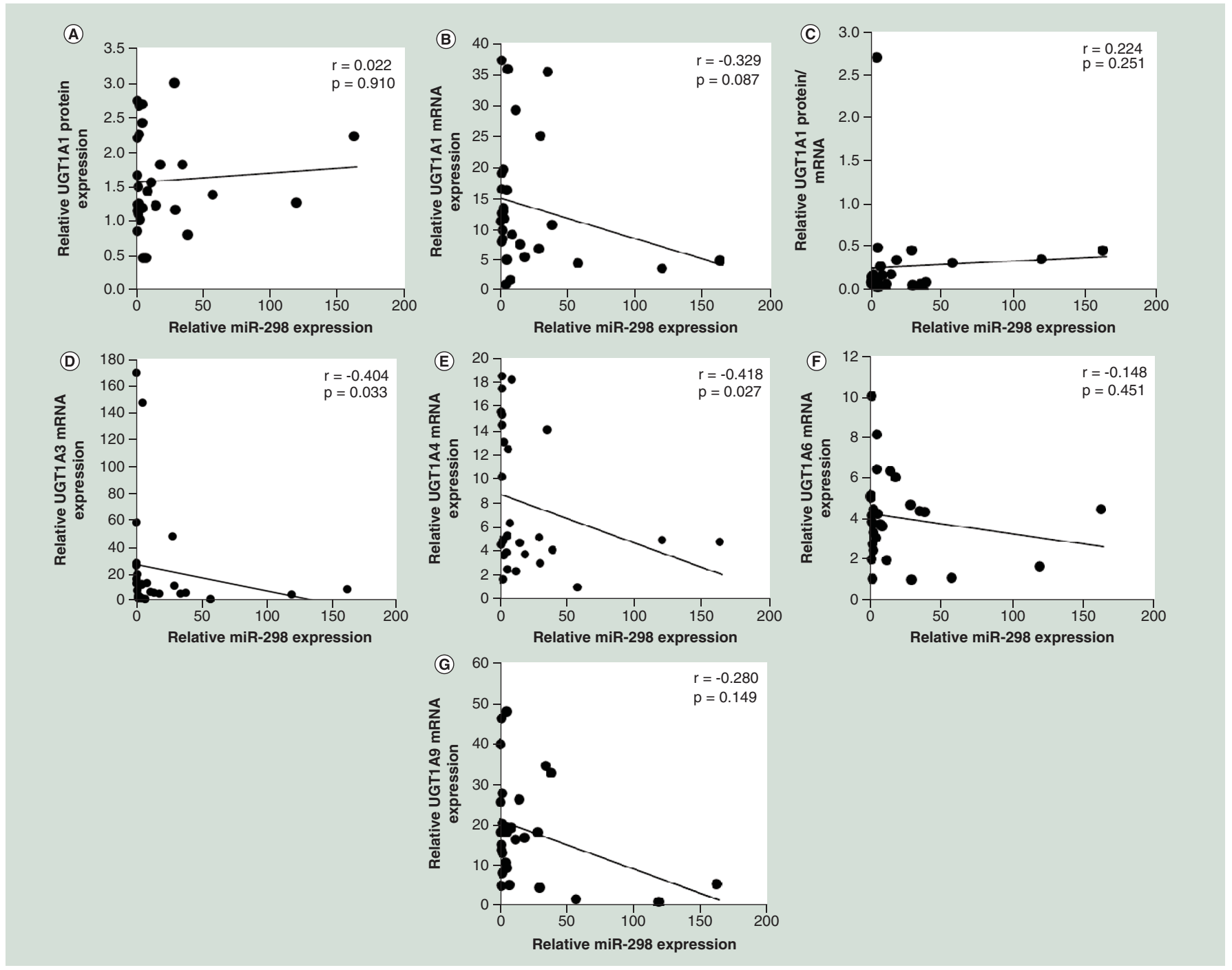

Figure 7. Analyses of correlations between expression levels of UGT1A and miR-298 in Chinese Han liver samples $(n=28)$. No correlation exists between miR-298 and UGT1A1 protein (A), mRNA (B) or protein/mRNA (C) level. The miR-298 levels were inversely correlated with UGT1A3 (D) or UGT1A4 (E) mRNA level. miR-298 was not correlated with UGT1A6 (F) or UGT1A9 (G). Levels of UGT1A mRNA and miR-298 were quantified in normal liver samples by qRT-PCR and normalized to GAPDH and U6 snRNA, respectively. Dots represent the mean \pm SEM of three independent replicates.

qRT-PCR: Quantitative real-time PCR; SEM: Standard error of the mean.

UGT1A1 expression via mRNA degradation. miR-298 overexpression led to a decrease in the mRNA levels of UGT1A1, UGT1A3, UGT1A4 and UGT1A9 in LS174T cells; however, no difference in UGT1A6 expression was observed. This indicates that the post-transcriptional regulation of $U G T 1 A$ by miR-298 has subtype specificity, even though the UGT1A family shares a common $3^{\prime}$-UTR. Similarly, miR-491 is not involved in regulation of the subtypes of $U G T 1 A$ [29]. As shown in previous studies, changes in mRNA secondary structure lead to altered miRNA regulation [35-37]. In the present study, we report a unique secondary structure for different UGT1A isoforms (Figure 9). In addition, we showed that the secondary structure of the miR-298-binding site for each UGT1A isoform was unique (Figure 9). These findings are mainly attributed to the specific UGT1A mRNA secondary structure, which potentially interferes with the interaction between miR-298 and UGT1A. Additionally, the mechanisms underlying regulation by miRNA involve mRNA degradation, which causes a reduction in mRNA expression level. miRNA regulation additionally involves the inhibition of translation, which leads to a decrease in the overall translational efficiency of the target mRNA. As both mechanisms play a role in reducing protein output, Takagi et al. proposed the concept of 'translation efficiency' to minimize transcription differences when evaluating 


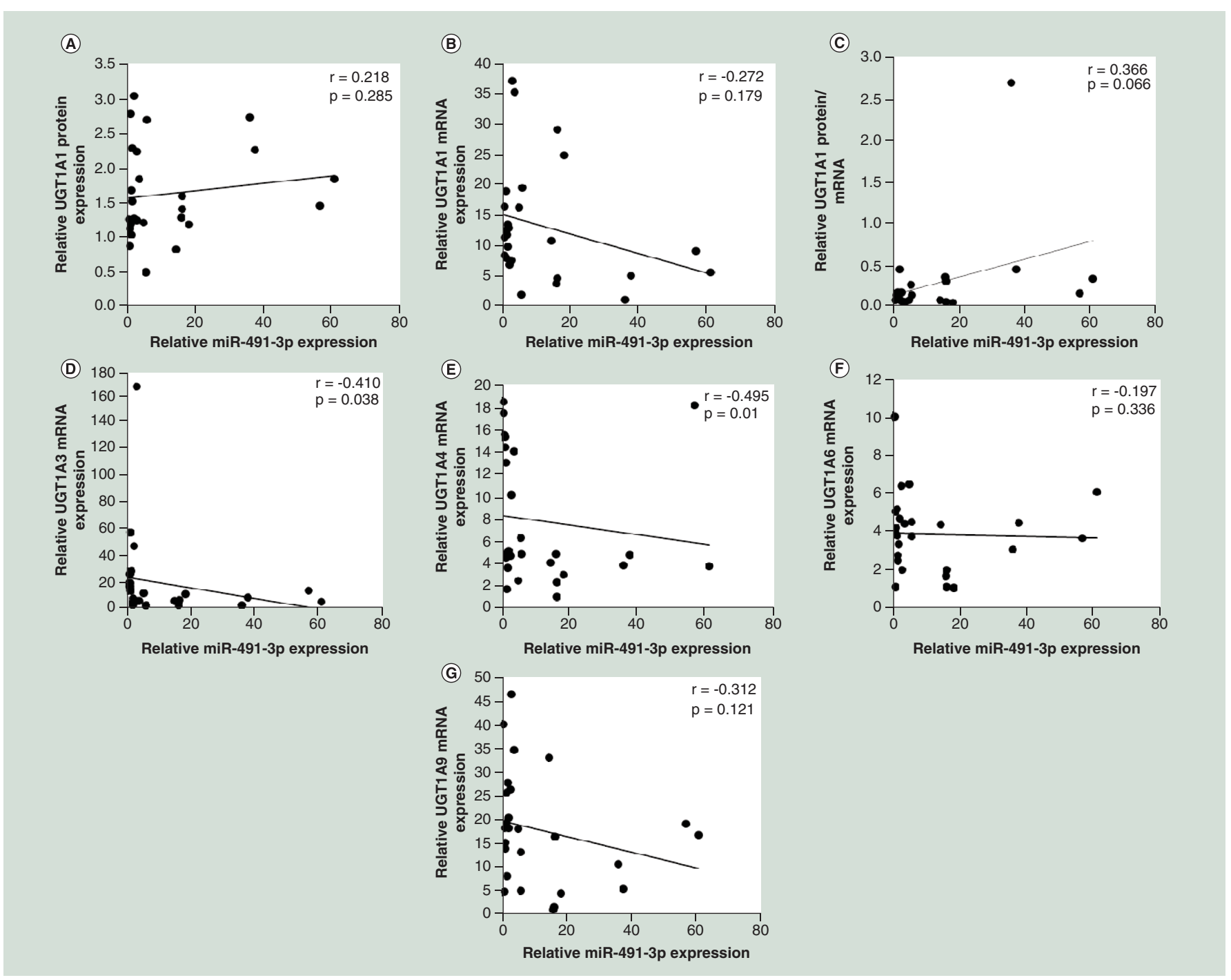

Figure 8. Analyses of correlations between expression levels of UGT1A and miR-491-3p in Chinese Han liver samples ( $n=28$ ). No correlation exists between miR-491-3p and UGT1A1 protein (A), mRNA (B) or protein/mRNA (C) level. The miR-491-3p levels were inversely correlated with UGT1A3 (D) or UGT1A4 (E) mRNA level. miR-491-3p was not correlated with UGT1A6 (F) or UGT1A9 (G). Levels of UGT1A mRNA and miR-491-3p were quantified in normal liver samples by qRT-PCR and normalized to GAPDH and U6 snRNA, respectively. Dots represent the mean \pm SEM of three independent replicates. qRT-PCR: Quantitative real-time PCR; SEM: Standard error of the mean.

the post-transcriptional regulation by miRNAs [38]. Oda et al. also reported that the two mechanisms can occur during the miR-34a mediation of RXR [39]. The miR-298-mediated change in the expression level of UGT1A1 can disrupt the normal balance in gene transcription. To thoroughly clarify which mechanisms underlying the regulation of $U G T 1 A 1$ by miR-298, additional studies are required. Moreover, the subtype-specific regulatory effects of miR-298 are putatively attributed to different mechanisms. Further studies of the effect of miR-298 on the protein expression of UGT1A3, UGT1A4, UGT1A6 and UGT1A9 levels are necessary for confirmation. The regulation of $U G T 1 A$ by miR-298 additionally occurs in a cell-specific manner. miRNAs act as genetic switches or fine tuners in the regulation of target gene expression, and this activity is dependent on the concentration of the targeted mRNA and that of the miRNA. It is likely that the differences in the overall effects of miR-298 on UGT1A in HepG2 and LS174T cells arise from different relative expression levels of UGT1A and miR-298. Dluzen et al. [29] reported that the cell-specific effects of miR-491-3p on UGT1A1 in HuH-7 and HepG2 cells may be attributed to the higher miR-491-3p/UGT1A1 expression ratio in HepG2 cells relative to HuH-7 cells. Cell-specific regulation is not a rare phenomenon; for example, a previous study showed that miR-27b only reduced the luciferase activity of 


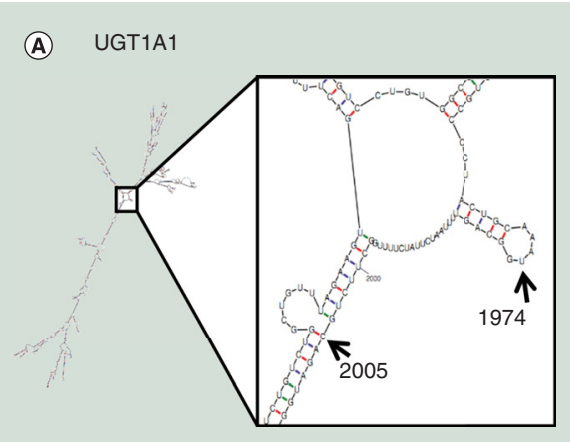

(D)

UGT1A6
(B) UGT1A3

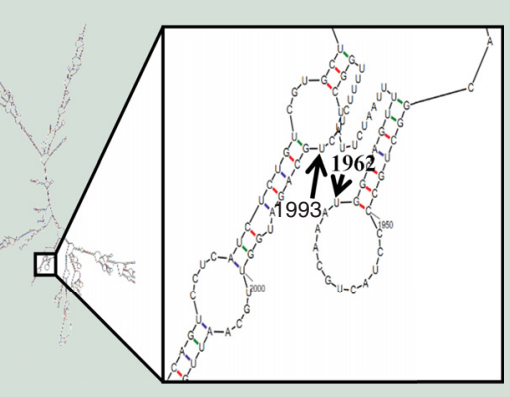

(C) UGTIA4

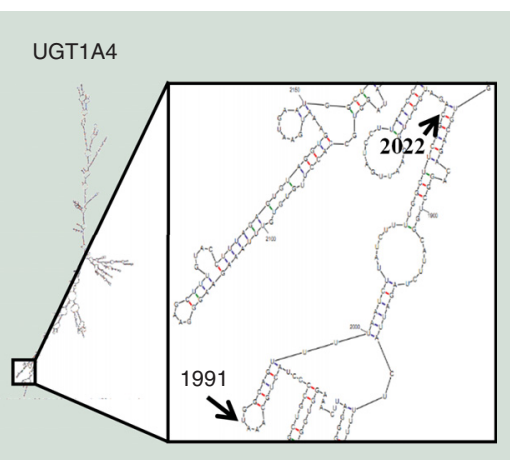

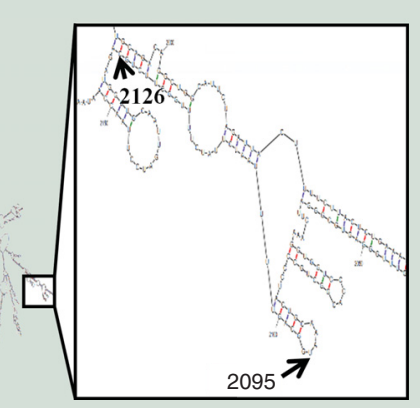

(E) UGT1A9

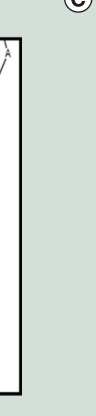

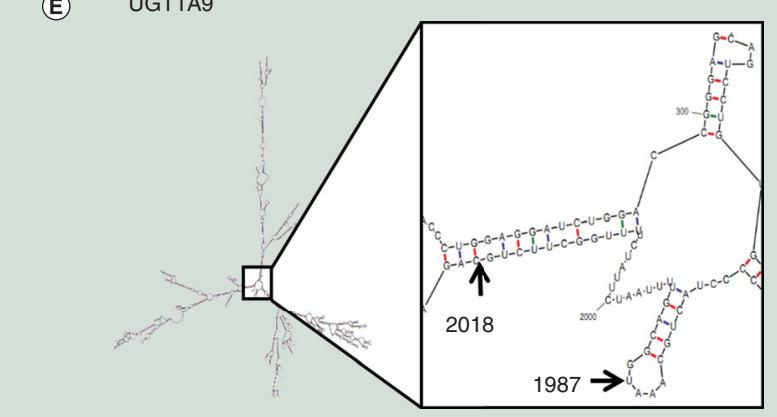

Figure 9. The secondary structures of UGT1A mRNA as assessed by the mfold Web Server. mfold Web Server (http://unafold.rna.albany.edu/?q=mfold) was used to predict the mRNA secondary structures for each hepatic UGT1A isoform. Secondary structures of miR-298 MRE region are enlarged at right. The numbering refers to the $5^{\prime}$ end of mRNA as 1 . The miR-298 MRE sequence is located from +1974 to +2005 in UGT1A1 mRNA (A), +1962 to 1993 in UGT1A3 mRNA (B), +1991 to +2022 in UGT1A4 mRNA (C), +2095 to +2126 in UGT1A6 mRNA (D) and +1987 to +2018 in UGT1A9 mRNA (E).

pGL3/1B1MRE $\times 6$ vector in Jurkat cells, whereas the luciferase activity of the pGL3/miR-27b, pGL3/1B1MRE $\times 3, \mathrm{pGL} 3 / 1 \mathrm{~B} 1 \mathrm{MRE} \times 6$ and pGL3/1B1MRE vectors was decreased in MCF-7 cells [40]. When HepG2 and $\mathrm{HuH}-7$ cells were treated with etoposide, miR-34a expression levels increased in HepG2 cells but not in HuH-7 cells [39]. Other genes such as that PPAR $\alpha$ exhibit cell-specific regulation; PPAR $\alpha$ is regulated by miR-21 and miR-27b in HuH-7 cells and by miR-141 in HepG2 cells [41,42].

In the present human liver specimens, inverse correlations were observed between UGT1A3 and UGT1A4 mRNA levels and miR-298 expression, which was consistent with the in vitro results. However, no correlation was found between miR-298 and the expression level of UGT1A1, although the present study showed a significant difference in the expression level of the UGT1A isoform. Similar to the effects observed in the cell lines, these results might be attributed to differences in the relative expression of each UGT1A isoform in the human liver. The regulation of target mRNA by miRNAs is thought to involve a complex network [43] in which a single gene may be regulated by multiple miRNAs, and a single miRNA regulates different genes. Furthermore, the overall expression level of UGT1A may rely on multiple miRNAs.

Moreover, previous studies have shown that UGT1A activity is additionally modified by post-transcriptional mechanisms such as phosphorylation, acetylation and protein-protein interactions with other factors or coregulators $[14,44-46]$. These findings suggest that UGT1A activity may be controlled by multiple pathways.

In addition to miRNA-mediated regulation, other factors, such as single nucleotide polymorphisms, transcription factors, DNA methylation and histone modification, contribute to the interindividual variability in UGTIA expression [14,16,47-49]. It is likely that miR-298 acts more as a participant than as a master in the complex UGT1A-regulatory network.

Ethnic variation is a confounding factor that potentially leads to controversial conclusions in scientific research and clinical investigations. Therefore, we investigated the effect of miR-491-3p on UGT1A expression specifically in the Chinese Han population. In the present human liver samples, an inverse correlation was observed between expression of UGT1A3 and that of miR-491-3p, which is consistent with the findings of previous studies [29]. A similar correlation was observed between UGTIA4 and miR-491-3p. However, no such association was observed 
for UGT1A6; this finding was inconsistent with that of Dluzen et al. This discrepancy may be attributable to several factors. The main contributing factor may be the difference in the populations studied. In the present work, all samples were obtained from Han Chinese subjects, whereas Dluzen et al. analyzed liver tissue obtained from American individuals. In addition, the techniques used to quantify the expression of miR-491-3p differed between the two studies. In the present work, we employed miRCURY LNA technology, which has high sensitivity and specificity, whereas Dluzen et al. used Taqman technology.

In summary, the present study demonstrates that miR-298 is involved in the post-transcriptional regulation of $U G T 1 A$. Similar to the results of a previous study, our findings indicate that miR-491-3p downregulates the expression of $U G T 1 A$ in the Chinese population. Taken together, these results confirm that miRNAs play a role in the regulation of $U G T 1 A$ expression. Further large-scale screening studies are warranted to systematically analyze the effects of miRNAs on $U G T 1 A$ expression. Furthermore, the role of miRNAs in regulating the tissue-specific expression of $U G T 1 A$ requires further elucidation.

\section{Summary points}

miRNAs are involved in the regulation of UGT1A

- UGT1A1 protein levels are not associated with UGT1A1 mRNA levels in livers from Han Chinese population.

- Bioinformatic analysis identifies UGT1A as a potential miRNA target.

- The potential recognition element of miR-298 was identified.

miR-298 altered the expression of UGT1A in HepG2 \& LS174T cells

- Transfection of miR-298 mimic into cells repressed UGT1A expression.

- Transfection of miR-298 inhibitor into cells increased UGT1A expression.

- The regulation of UGT1A by miR-298 occurs in a cell-specific manner.

miR-298 \& miR-491-3p are involved in the regulation of UGT1A in livers from Han Chinese population $(n=28)$

- Inverse correlations between miR-298 and UGT1A3 or UGT1A4 mRNA levels, as well as between miR-491-3p and UGT1A3 or UGT1A4 mRNA, were observed in the livers of the Han Chinese population.

mRNA secondary structure contributes the subtype specificity regulation of UGT1A by miRNA

- Each UGT1A isoforms possesses a unique secondary structure.

- The secondary structure of the miR-298-binding site in each UGT1A isoform was unique.

- Our findings extend the knowledge of postregulation of UGT1A by miRNA. miR-298 and miR-491-3p downregulate UGT1A expression in vitro and in the Han Chinese population.

Financial \& competing interests disclosure

This work was supported by the National Natural Science Foundation of China (grant number 81173127) and the Joint Funds of the National Natural Science Foundation of China-Henan province (grant number U1604163). The authors have no other relevant affiliations or financial involvement with any organization or entity with a financial interest in or financial conflict with the subject matter or materials discussed in the manuscript apart from those disclosed.

No writing assistance was utilized in the production of this manuscript.

Ethical conduct of research

The authors state that they have obtained appropriate institutional review board approval or have followed the principles outlined in the Declaration of Helsinki for all human or animal experimental investigations. In addition, for investigations involving human subjects, informed consent has been obtained from the participants involved.

\section{References}

1 Rowland A, Miners JO, Mackenzie PI. The UDP-glucuronosyltransferases: their role in drug metabolism and detoxification. Int. J. Biochem. Cell B. 45(6), 1121-1132 (2013).

2 Mackenzie PI, Bock KW, Burchell B et al. Nomenclature update for the mammalian UDP glycosyltransferase (UGT) gene superfamily. Pharmacogenet. Genomics 15(10), 677-685 (2005).

3 Gong QH, Cho JW, Huang T et al. Thirteen UDP-glucuronosyltransferase genes are encoded at the human UGT1 gene complex locus. Pharmacogenetics 11(4), 357-368 (2001).

4 Guillemette C, Levesque E, Harvey M, Bellemare J, Menard V. UGT genomic diversity: beyond gene duplication. Drug Metab. Rev. 42(1), 24-44 (2010). 
5 Ohno S, Nakajin S. Determination of mRNA expression of human UDP-glucuronosyltransferases and application for localization in various human tissues by real-time reverse transcriptase-polymerase chain reaction. Drug Metab. Dispos. 37(1), 32-40 (2008).

6 Court MH, Zhang X, Ding X et al. Quantitative distribution of mRNAs encoding the 19 human UDP-glucuronosyltransferase enzymes in 26 adult and 3 fetal tissues. Xenobiotica 42(3), 266-277 (2012).

7 Liu W, Ramirez J, Gamazon ER et al. Genetic factors affecting gene transcription and catalytic activity of UDP-glucuronosyltransferases in human liver. Hum. Mol. Genet. 23(20), 5558-5569 (2014).

8 Tukey RH, Strassburg CP. Human UDP-glucuronosyltransferases: metabolism, expression, and disease. Annu. Rev. Pharmacol. 40(1), 581-616 (2000).

9 Bosma PJ, Chowdhury JR, Bakker C et al. The genetic basis of the reduced expression of bilirubin UDP-glucuronosyltransferase 1 in Gilbert's syndrome. N. Engl. J. Med. 333(18), 1171-1175 (1995).

10 Kiang T, Ensom M, Chang T. UDP-glucuronosyltransferases and clinical drug-drug interactions. Pharmacol. Ther. 106(1), 97-132 (2005).

11 Court MH, Duan SX, Moltke LL et al. Interindividual variability in acetaminophen glucuronidation by human liver microsomes: identification of relevant acetaminophen UDP-glucuronosyltransferase isoforms. J. Pharmacol. Exp. Ther. 299(3), 998-1006 (2001).

12 Kostrubsky SE. Phenobarbital and phenytoin increased acetaminophen hepatotoxicity due to inhibition of UDP-glucuronosyltransferases in cultured human hepatocytes. Toxicol. Sci. 87(1), 146-155 (2005).

13 Sugatani J. Function genetic polymorphism, and transcriptional regulation of human UDP-glucuronosyltransferase (UGT) 1A1. Drug Metab. Pharmacol. 28(2), 83-92 (2013).

14 Sugatani J, Uchida T, Kurosawa $\mathrm{M}$ et al. Regulation of pregnane X receptor (PXR) function and UGT1A1 gene expression by posttranslational modification of PXR protein. Drug Metab. Dispos. 40(10), 2031-2040 (2012).

15 Zheng B, Hu G, Yu J, Liu Z. Crigler-Najjar syndrome type II in a Chinese boy resulting from three mutations in the bilirubin uridine 5'-diphosphate-glucuronosyltransferase (UGT1A1) gene and a family genetic analysis. BMC Pediatr. 14(1), 267 (2014).

16 Oda S, Fukami T, Yokoi T, Nakajima M. Epigenetic regulation is a crucial factor in the repression of UGT1A1 expression in the human kidney. Drug Metab. Dispos. 41(10), 1738-1743 (2013).

17 Oda S, Fukami T, Yokoi T, Nakajima M. Epigenetic regulation of the tissue-specific expression of human UDP-glucuronosyltransferase (UGT) 1A10. Biochem. Pharmacol. 87(4), 660-667 (2014).

18 Izukawa T, Nakajima M, Fujiwara R et al. Quantitative analysis of UDP-glucuronosyltransferase (UGT) 1A and UGT2B expression levels in human livers. Drug Metab. Dispos. 37(8), 1759-1768 (2009).

19 Ohtsuki S, Schaefer O, Kawakami H et al. Simultaneous absolute protein quantification of transporters, cytochromes $\mathrm{P} 450$, and UDP-glucuronosyltransferases as a novel approach for the characterization of individual human liver: comparison with mRNA levels and activities. Drug Metab. Dispos. 40(1), 83-92 (2011).

20 Koturbash I, Tolleson WH, Guo L et al. microRNAs as pharmacogenomic biomarkers for drug efficacy and drug safety assessment. Biomark. Med. 9(11), 1153-1176 (2015).

21 Duursma AM, Kedde M, Schrier M, le Sage C, Agami R. miR-148 targets human DNMT3b protein coding region. RNA 14(5), 872-877 (2008).

22 Ørom UA, Nielsen FC, Lund AH. MicroRNA-10a binds the 5'UTR of ribosomal protein mRNAs and enhances their translation. Mol. Cell 30(4), 460-471 (2008).

23 Garzon R, Marcucci G, Croce CM. Targeting microRNAs in cancer: rationale, strategies and challenges. Nat. Rev. Drug Discov. 9(10), 775-789 (2010).

24 Lewis BP, Burge CB, Bartel DP. Conserved seed pairing, often flanked by adenosines, indicates that thousands of human genes are microRNA targets. Cell 120(1), 15-20 (2005).

25 Yan L, Liu J, Zhao Y et al. Suppression of miR-628-3p and miR-641 is involved in rifampin-mediated CYP3A4 induction in HepaRG cells. Pharmacogenomics 18(1), 57-64 (2017).

26 Wei Z, Jiang S, Zhang Y et al. The effect of microRNAs in the regulation of human CYP3A4: a systematic study using a mathematical model. Sci. Rep. 4(1), 4283 (2015).

27 Shi Y, Liu Y, Wei Z et al. Hsa-miR-27a is involved in the regulation of CYP3A4 expression in human livers from Chinese Han population. Pharmacogenomics 16(12), 1379-1386 (2015).

28 He Y, Chevillet JR, Liu G, Kim TK, Wang K. The effects of microRNA on the absorption, distribution, metabolism and excretion of drugs. Br. J. Pharmacol. 172(11), 2733-2747 (2015).

29 Dluzen DF, Sun D, Salzberg AC et al. Regulation of UDP-glucuronosyltransferase 1A1 expression and activity by microRNA 491-3p. J. Pharmacol. Exp. Ther. 348(3), 465-477 (2014).

30 Wei ZY, Chen MJ, Zhang YT et al. No correlation of hsa-miR-148a with expression of PXR or CYP3A4 in humanlivers from Chinese Han population. PLoS ONE 8(3), e59141 (2013).

31 Strassburg CP. Pharmacogenetics of Gilbert's syndrome. Pharmacogenomics 9(6), 703-715 (2008). 
32 Tracy TS, Chaudhry AS, Prasad B et al. Interindividual variability in cytochrome P450-mediated drug metabolism. Drug Metab. Dispos. 44(3), 343-351 (2016).

33 Sim SC, Kacevska M, Ingelman-Sundberg M. Pharmacogenomics of drug-metabolizing enzymes: a recent update on clinical implications and endogenous effects. Pharmacogenomics J. 13(1), 1-11 (2012).

34 Dluzen DF, Lazarus P. MicroRNA regulation of the major drug-metabolizing enzymes and related transcription factors. Drug Metab. Rev. 47(3), 320-334 (2015).

35 Ramamoorthy A, Li L, Gaedigk A et al. In silico and in vitro identification of microRNAs that regulate hepatic nuclear factor 4 expression. Drug Metab. Dispos. 40(4), 726-733 (2012).

36 Haas U, Sczakiel G, Laufer S. MicroRNA-mediated regulation of gene expression is affected by disease-associated SNPs within the 3'-UTR via altered RNA structure. RNA Biol. 9(6), 924-937 (2014).

37 Long D, Lee R, Williams P et al. Potent effect of target structure on microRNA function. Nat. Struct. Mol. Biol. 14(4), 287-294 (2007).

38 Takagi S, Nakajima M, Mohri T, Yokoi T. Post-transcriptional regulation of human pregnane X receptor by micro-RNA affects the expression of cytochrome P450 3A4. J. Biol. Chem. 283(15), 9674-9680 (2008).

39 Oda Y, Nakajima M, Tsuneyama K et al. Retinoid X receptor $\alpha$ in human liver is regulated by miR-34a. Biochem. Pharmacol. 90(2), 179-187 (2014).

40 Tsuchiya Y, Nakajima M, Takagi S, Taniya T, Yokoi T. MicroRNA regulates the expression of human cytochrome P4501B1. Cancer Res. 66(18), 9090-9098 (2006).

41 Kida K, Nakajima M, Mohri T et al. PPAR $\alpha$ is regulated by miR-21 and miR-27b in human liver. Pharm. Res. 28(10), 2467-2476 (2011).

42 Wei Hu, Wang X, Ding X et al. MicroRNA-141 represses HBV replication by targeting PPARA. PLoS ONE 7(3), e34165 (2012).

43 He Y, Chevillet JR, Liu G, Kim TK, Wang K. The effects of microRNA on the absorption, distribution, metabolism and excretion of drugs. Br. J. Pharmacol. 172(11), 2733-2747 (2015).

44 Riches Z, Collier AC. Posttranscriptional regulation of uridine diphosphate glucuronosyltransferases. Expert. Opin. Drug Met. 11(6), 949-965 (2015).

45 Basu NK, Kole L, Owens IS. Evidence for phosphorylation requirement for human bilirubin UDP-glucuronosyltransferase (UGT1A1) activity. Biochem. Biophys. Res. Commun. 303(1), 98-104 (2003).

46 Williams JA, Hyland R, Jones BC et al. Drug-drug interactions for UDP-glucuronosyltransferase substrates: a pharmacokinetic explanation for typically observed low exposure (AUCI/AUC) ratios. Drug Metab. Dispos. 32(11), 1201-1208 (2004).

47 Cheng L, Li M, Hu J et al. UGT1A1*6 polymorphisms are correlated with irinotecan-induced toxicity: a system review and meta-analysis in Asians. Cancer Chemother. Pharm. 73(3), 551-560 (2014).

48 Hu DG, Meech R, McKinnon RA, Mackenzie PI. Transcriptional regulation of human UDP-glucuronosyltransferase genes. Drug Metab. Rev. 46(4), 421-458 (2014).

$49 \mathrm{Nie} \mathrm{Y}, \mathrm{He} \mathrm{H}, \mathrm{Li}$ J et al. Hepatic expression of transcription factors affecting developmental regulation of UGT1A1 in the Han Chinese population. Eur. J. Clin. Pharmacol. 73(1), 29-37 (2016). 
(

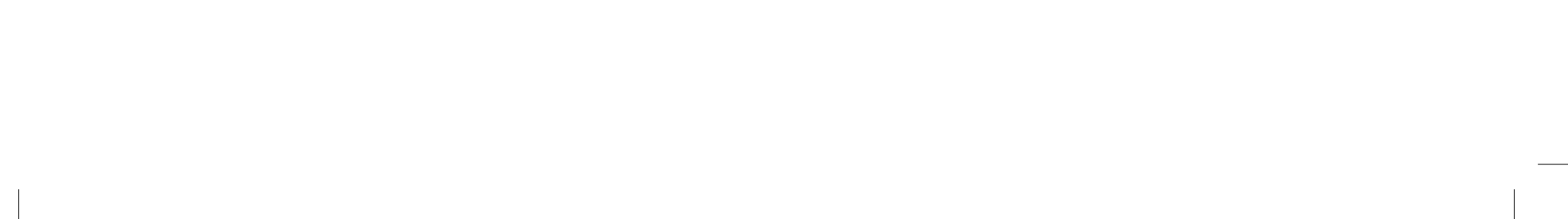




\section{Plant homeodomain finger protein 2 as a novel IKAROS target in acute lymphoblastic leukemia}

Zheng Ge*1,2, Yan Gu1 ${ }^{1}$, Qi Han ${ }^{3}$, Justin Sloane ${ }^{4,5}$, Qinyu Ge ${ }^{6}$, Goufeng Gao7, Jinlong

$\mathrm{Ma}^{1,2}$, Huihui Song ${ }^{1}$, Jiaojiao $\mathrm{Hu}^{1,2}$, Baoan Chen ${ }^{1,2}$, Sinisa Dovat ${ }^{\#, 2,5}$ \& Chunhua Song ${ }^{\$, 2,5}$

${ }^{1}$ Department of Hematology, Zhongda Hospital, Medical School of Southeast University, Southeast University Institute of

Hematology, Nanjing 210009, China

${ }^{2}$ International Cooperative Leukemia Group \& International Cooperative Laboratory of Hematology, Zhongda Hospital, Medical

School of Southeast University, Nanjing 210009, China

${ }^{3}$ Department of Hematology, The First Affiliated Hospital of Nanjing Medical University, Jiangsu Province Hospital, Nanjing 210029,

China

${ }^{4}$ Department of Obstetrics \& Gynecology, Abington Jefferson-Health, Abington, PA 19001, USA

${ }^{5}$ Department of Pediatrics, Pennsylvania State University Medical College, Hershey, PA 17033, USA

${ }^{6}$ State Key Laboratory of Bioelectronics, School of Biological Science \& Medical Engineering, Southeast University, Nanjing 210096, China

${ }^{7}$ Department of Pathology \& Laboratory Medicine, University of California-Davis Medical Center, Sacramento, CA 95817, USA

* Author for correspondence: Janege879@hotmail.com

\# Author for correspondence: sdovat@pennstatehealth.psu.edu

\$ Author for correspondence: csong@pennstatehealth.psu.edu

Aim: Clinical significance of plant homeodomain finger 2 (PHF2) expressions is explored in acute lymphoblastic leukemia (ALL) patients. Methods: mRNA level was examined by qPCR. The retroviral gene expression, shRNA knockdown and chromatin-immunoprecipitation are used to observe IKAROS regulation on PHF2 transcription. Results: PHF2 expression is significantly reduced in subsets of ALL patients, and $P H F 2^{\text {low }}$ expression correlates with leukemia cell proliferation and an elevation of several poor prognostic markers in B-cell ALL. IKAROS directly promotes PHF2 expression and patients with IKAROS deletion have significantly lower PHF2 expression. Casein kinase II (CK2) inhibitor significantly promotes PHF2 expression in an IKAROS-dependent manner, and casein kinase II inhibitor treatment also results in an increase of PHF2 expression and enrichment of IKAROS and H3K4me3 at PHF2 promoter in primary cells. Conclusion: Our results demonstrate that the IKAROS promotes $P H F 2$ expression, and suggest that $P H F 2^{\text {low }}$ expression works with the IKAROS gene deletion to drive oncogenesis of ALL.

First draft submitted: 30 July 2017; Accepted for publication: 26 September 2017; Published online: 10 October 2017

Keywords: acute lymphoblastic leukemia • histone modification •IKZF1 • PHF2

Histone demethylases and their role in the regulation of post-translational modifications of chromatin have greatly contributed to our understanding of epigenetics in tumorigenesis. Plant homeodomain finger 2 (PHF2) (Lysine demethylase $7 \mathrm{C}[\mathrm{KDM} 7 \mathrm{C}])$ is a member of the Jumonji $\mathrm{C}(\mathrm{JmjC})$ family and contains a plant homeodomain finger and a JmjC domain. PHF2 demethylates H3K9me2 (lysyl dimethylation), which, subsequently participates in epigenetic regulation of transcription [1].

PHF2 is a positive epigenetic regulator and is associated with tumor suppression in several types of cancer. PHF2 gene deletion, or hypermethylation in its promoter region, is associated with breast cancer, whereas its downregulation is associated with head and neck cancers [2,3]. PHF2 positively regulates the expression of genes such as $H N F 4, C E B P \alpha$ and $N F-\kappa B$, and subsequently aids with the expression of genes driven by these factors [4-6]. PHF2 is also in association with p53 which is involved in tumor suppression in human cancer [7]. It is also reported that PHF2 promotes the differentiation of tumor-initiating cells and induces the loss of tumor-initiating ability in the cells [8]. Thus, PHF2 is well recognized as a tumor suppressor. 
Many reports indicate that histone demethylases are aberrantly expressed in human cancers and may potentially provide additional therapeutic targets in the treatment of cancer [9-11], although the underlying mechanism of neoplastic transformation remains unknown. For example, JMJD3 is highly expressed in T-cell ALL (T-ALL) [12]; $K D M 5 B$ is highly expressed in acute lymphoblastic leukemia (ALL) [13]; PHF8 promotes breast cancer oncogenesis and governs the response to retinoic acid in acute myeloid leukemia (AML) [14,15]; and PHF2 is downregulated in colon cancer [4,7] and breast cancer [2]. It is also reported that low PHF2 expression is associated with increased aggressiveness of the clear cell variant of renal cell carcinoma and is a poor prognostic factor [16]. Until now, however, there have been no reports about the PHF2 expression in leukemia patients and its roles in oncogenesis in ALL.

Genetic alterations of $I K Z F 1$ encoding, the lymphoid transcription factor IKAROS, are a hallmark of high-risk B-progenitor ALL such as BCR-ABL1 positive $(\mathrm{Ph}+)$ and $\mathrm{Ph}$-like $\mathrm{ALL}$, and are associated with poor outcome, even in the era of contemporary chemotherapy incorporating tyrosine kinase inhibitors in the treatment of $\mathrm{Ph}+$ ALL [17]. Recently, we reported the IKAROS global binding profile in ALL cells and found that IKAROS regulates the expression of its targets through chromatin remodeling in ALL [18-21]. Our chromatin immunoprecipitation assays with sequencing (ChIP-seq) data showed IKAROS binding peaks in the promoter region of PHF2. We also found that casein kinase II (CK2) inhibitors increase the tumor suppressor activity of IKAROS and act as a functional activator of the IKZF1 gene product [18-20]. However, it is still unclear whether and how IKAROS regulates $P H F 2$ expression.

Here, we examined PHF2 expression in ALL patients, and analyzed the correlation between PHF2 expression and several high-risk markers in ALL patients. We also identified that PHF2 is a direct target of IKAROS, which binds to and promotes PHF2 expression in ALL leukemic cells.

CX-4945 is an inhibitor of CK2. We reported that CK2-induced IKAROS phosphorylation result in the IKAROS inactivation. CK2 activity is higher in leukemic cells, and CK2 inhibitor reduces the IKAROS phosphorylation and restores IKAROS tumor suppressor activity [18-21]. Here, we found that CX-4945 treatment increases PHF2 expression, whereas $I K Z F 1$ knockdown blocks these CX-4945-induced changes. In addition, we found that deletion of the IKZF1 gene is significantly associated with low PHF2 expression in ALL. We also found that IKAROS promotes PHF2 expression by chromatin remodeling through enrichment of H3K4me3 to the promoter. Our findings indicate that PHF2 is significantly downregulated in ALL, and that an IKZF1 deletion may be responsible for the low expression of PHF2 in high-risk ALL. PHF2 low expression may work in conjunction with IKZF1 deletion to drive oncogenesis in ALL. The significance of the correlation may also be valuable in an integrated prognostic model for high-risk ALL although it requires large prospective trials to confirmation.

\section{Materials \& methods}

\section{Subjects \& samples}

Subjects with newly diagnosed ALL (age 12-77 years old) were recruited between June 2008 and June 2016. There was a total of 164 patients (107 B-cell and 57 T-cell ALL) who were recruited at the First Affiliated Hospital of Nanjing Medical University and Zhongda Hospital Southeast University. Diagnoses were based on the WHO Diagnosis and Classification of ALL (2008). The study was approved by the Ethics Committee of the First Affiliated Hospital of Nanjing Medical University and Zhongda Hospital Southeast University, Nanjing, China.

\section{Therapy}

The therapy protocol has been previously published (CALLG2008) [22]. Induction was with vincristine, daunorubicin, cyclophosphamide, L-asparaginase, and prednisone-based regiments. Early consolidation used cyclophosphamide, cytarabine, thioguanine, high-dose methotrexate/L-asparaginase and mitoxantrone. Late consolidation used vincristine, daunorubicin, L-asparaginase, prednisone; cyclophosphamide, vincristine, cytarabine, epipodophyllotoxin and dexamethasone; or high-dose methotrexate/L-asparaginase, epipodophyllotoxin and cytarabine. Maintenance therapy used 6-mercaptopurine and methotrexate. Subjects with BCR/ABL1-positive ALL received Imatinib starting day 15 of introduction therapy.

\section{Cytogenetic \& molecular analyses}

Cytogenetics and detection of the most common IKZF1 deletion, IKAROS 6 (Ik6), were analyzed as previously described [20]. qPCR was performed on StepOne Plus Real-time PCR system (Applied Biosystem-Thermofisher, CA, USA). Gene expression values of genes of interest (GOI) were achieved in each sample by a formula derived from a scatter graph of cycle threshold $(\mathrm{Ct})$ values from serial dilutions of a template standard as described [20,23]. 
Expression levels of GOIs were normalized to housekeeping genes expressed as gene expression value of GOI/18s rRNA. Subjects were allocated into high or low PHF2 expression cohorts (4th quartile vs 1st-3rd quartiles) with a cut-off value determined by SPSS 20.0 (IBM, NY, USA) [20].

The qPCR for PHF2 expression was analyzed as above in Nalm6, CEM cells and primary ALL cells. Results were normalized to those obtained with $18 s \mathrm{~s} R N A$ and presented as fold induction over vector controls. Primers: $18 \mathrm{~s}$ $r R N A$, sense: 5'-GTAACCCGTTGAACCCCATT-3', antisense: 5' - CCATCCAATCGGTAGTAGCG-3'; PHF2 sense: 5' - CAAGCGGGTCCTCAACGT-3', antisense: 5' - CAAGCGGGTCCTCAACGT -3' .

\section{Cell culture, plasmids \& retroviral gene transfer}

The Nalm6 cell line has been previously described [20]. The CCRF-CEM (CEM) cell lines were obtained from the American Type Culture Collection (VA, USA). Cells were cultured in RPMI-1640 medium (Cellgro, MA, USA) supplemented with 10\% fetal bovine serum (Hyclone, UT, USA). HEK 293T cells were cultured in DMEM (Cellgro) supplemented with $10 \%$ fetal calf serum and $1 \%$ L-glutamine (Cellgro). Cells were incubated at $37^{\circ} \mathrm{C}$ in a humidified atmosphere with 5\% $\mathrm{CO}_{2}$. Primary human B- and T-cell ALL cells were cultured in RPMI-1640 medium supplemented with 10\% fetal bovine serum (GE-Hyclone, UT, USA). CX-4945 was purchased from Sigma (MO, USA). Cells were cultured with or without CX-4945 and collected for total RNA isolation. Human IKZF1 retroviral construct and retroviral production have previously been described [18,20,24-26].

\section{Quantitative chromatin immunoprecipitation}

Quantitative chromatin immunoprecipitation (ChIP) assays were performed by incubating chromatin with antibodies against IKAROS [18-20], H3K4me3 and HDAC1 (Abcam, MA, USA), Mi2 (CA, USA) or normal rabbit IgG (Abcam) as a control [18-20,26]. Enrichment of the ChIP sample over input was evaluated by qPCR with more than or equal to three replicates using specific primers in the promoter region of PHF2 (forward: 5'TGCTAGGAGACTGAGCCAGGTT-3', reverse: 5'-ACCCCTGCTCGGAATTCC-3') or no IKAROS-binding region (IKAROS-negative-binding control primers, forward: 5'- GCTCGAAACCACCCTGATCA-3', reverse: 5'TGCCGGCTAATTTTGTATTTTTAGT-3'). Relative concentration of the qPCR product is presented as the fold change of the level of DNA-IKAROS and DNA-H3K4me3 samples compared with controls.

\section{Luciferase assay}

The PHF2 promoter was cloned into pGL4.15 vector (Promega, WI, USA). The transient luciferase reporter assay was performed in HEK293T cells using the Promega luciferase assay reagents and measured with luminometer following the manufacture's instruction $[18,20,26]$. The firefly luciferase activities were calculated as fold change relative to values obtained from pGL4.15 vector only control cells, and expressed as a percentage of pcDNA3.1$I K Z F 1$ transfection-induced luciferase activity versus that of pcDNA3.1 vector. All transfection and reporter assays were performed independently, in triplicate, at least three-times.

For CX-4945 treatment, the cells are incubated with $20 \mu \mathrm{M} \mathrm{CX}-4945$ for $24 \mathrm{~h}$ after the transfection.

\section{IKZF1 shRNA knockdown}

Nalm6 and CEM cells were transiently transfected with human IKZF1 shRNA constructs in the green fluorescent protein vector (pGFP-v-RS) (OriGene, MD, USA) using the Neon Transfection System (Invitrogen, CA, USA). We used scrambled 29-mer shRNA cassette in the pGFP-v-RS vector as a control. Knockdown of IKAROS was confirmed by IKZF1 mRNA level $[18,20,26]$. Primers used for qPCR are 5'-GGCGCGGTGCTCCTCCT-3' (IKZF1-F) and 5'-TCCGACACGCCCTACGACA-3' (IKZF1-R).

\section{Statistical analyses}

Median differences between the cohorts were evaluated using a Mann-Whitney U-test. Frequency differences were analyzed using univariate and multivariate Cox models. Relapse-free survival (RFS) and overall survival (OS) were estimated by the Kaplan-Meier method and compared by log-rank test. The starting point for the observation time for OS was date of diagnosis. RFS was estimated for subjects achieving complete remission starting at the time remission was declared. Living subjects were censored for survival at last follow-up. Statistical analyses used SPSS version 20.0. Data were represented as mean values with bars representing the standard error of the mean. Data shown are representative of three independent experiments. Determinations of statistical significance were 


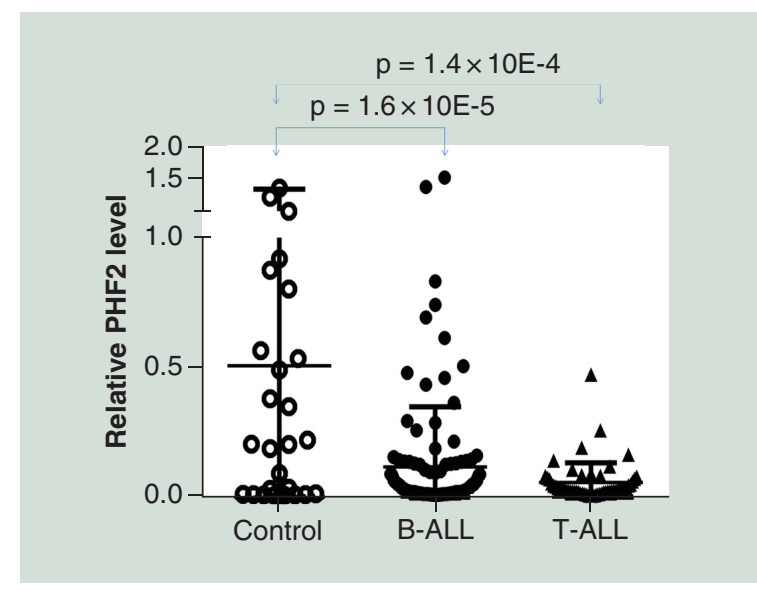

Figure 1. PHF2 expression in acute lymphoblastic leukemia.

Comparison of PHF2 expression in B-cell acute lymphoblastic leukemia and T-cell acute lymphoblastic leukemia with normal bone marrow controls.

\begin{tabular}{|c|c|c|c|c|c|}
\hline \multirow[t]{2}{*}{ Characteristics } & \multirow[t]{2}{*}{ PHF2 ${ }^{\text {high }}$} & \multirow[t]{2}{*}{ PHF2 low } & \multirow{2}{*}{$\begin{array}{l}\text { Univariate analyses } \\
\text { (Chi-square tests) } \\
p \text {-value }\end{array}$} & \multicolumn{2}{|c|}{ Multivariate analyses (multivariate Cox model) } \\
\hline & & & & $p$-value & HR $(95 \%$ Cl) \\
\hline IKZF1 deletion (Ik6) (\%) & 46.3 & 8.0 & 0.001 & 0.005 & $0.021(0.001-0.321)$ \\
\hline $\begin{array}{l}\text { Bone marrow blasts } \\
\text { (\%)median (range) }\end{array}$ & $90.4(38.0-100.0)$ & $82.4(28.0-96.0)$ & 0.001 & 0.021 & $0.001(0.000-0.335)$ \\
\hline $\begin{array}{l}\text { Spleen extramedullary } \\
\text { infiltration (\%) }\end{array}$ & 46.8 & 23.1 & 0.034 & 0.412 & $2.018(0.377-10.799)$ \\
\hline $\begin{array}{l}\mathrm{PLT}, \times 10^{9} / / \text { median } \\
\text { (range) }\end{array}$ & $32.0(2.0-292.0)$ & $62.0(4.0-292.0)$ & 0.048 & 0.143 & $1.008(0.997-1.019)$ \\
\hline $\begin{array}{l}\text { Stem cell marker } \mathrm{CD} 34^{+} \\
(\%)\end{array}$ & 82.3 & 60.0 & 0.021 & 0.728 & $1.325(0.271-6.484)$ \\
\hline Myeloid marker CD33 (\%) & 48.5 & 25.0 & 0.046 & 0.479 & $0.576(0.125-2.651)$ \\
\hline $\begin{array}{l}\text { Time to reach CR after } \\
\text { treatment }>4 \text { weeks (\%) }\end{array}$ & 48.7 & 19.0 & 0.015 & 0.006 & $0.119(0.026-0.549)$ \\
\hline
\end{tabular}

performed using a Student's $t$-test for comparison of two groups or analysis of variance for comparing more than two groups.

\section{Results}

Clinical \& laboratory variables in patients with high \& low PHF2 expression

PHF2 mRNA levels in bone marrow samples from adults with ALL, especially those with B-cell ALL (B-ALL), were significantly lower than those in healthy controls (Figure 1) and also in B-ALL (GSE7440 and GSE11877) and T-ALL (GSE10609, GSE26713) cohort studies (Supplementary Figure 1). We compared clinical and laboratory variables in subjects with B-ALL divided into cohorts with high or low PHF2 mRNA levels (Tables 1 and Supplementary Table 1). The B-ALL cohort with low PHF2 mRNA levels was associated with a high-median bone marrow blast percentage (90.4 vs $82.4 \%$; $\mathrm{p}=0.001)$, higher percentage of stem cell marker CD34 ${ }^{+}(82.3$ vs $60.0 \% ; \mathrm{p}=0.021$ ) and a higher myeloid marker CD33 (48.5 vs $25.0 \% ; \mathrm{p}=0.046$ ) in comparison to the high PHF2 expression cohort (Table 1 and Supplementary Table 1). The associated median bone marrow blast percentage was confirmed in multivariate analyses (Table 1). The low PHF2 expression cohort also had a higher frequency of Ik6 $(+)$, the most common IKZFI deletion ( 46.3 vs $8.0 \%$; $\mathrm{p}=0.001)$; a lower median platelet (PLT) count and a higher percentage of splenomegaly ( 46.8 vs $23.1 \%$; $\mathrm{p}=0.034$ ) (Table 1 ) compared with the high PHF2 expression cohort. The associated Ik6(+) percentages were also confirmed in multivariate analyses (Table 1$)$. There were no significant associations between clinical and laboratory variables in the low versus high PHF2 expression cohorts in subject with T-ALL (data not shown).

Time to complete remission $>4$ weeks after chemotherapy induction is considered a poor prognostic marker in patients with ALL. We found that our cohort of B-ALL patients with low PHF2 expression had a significantly 


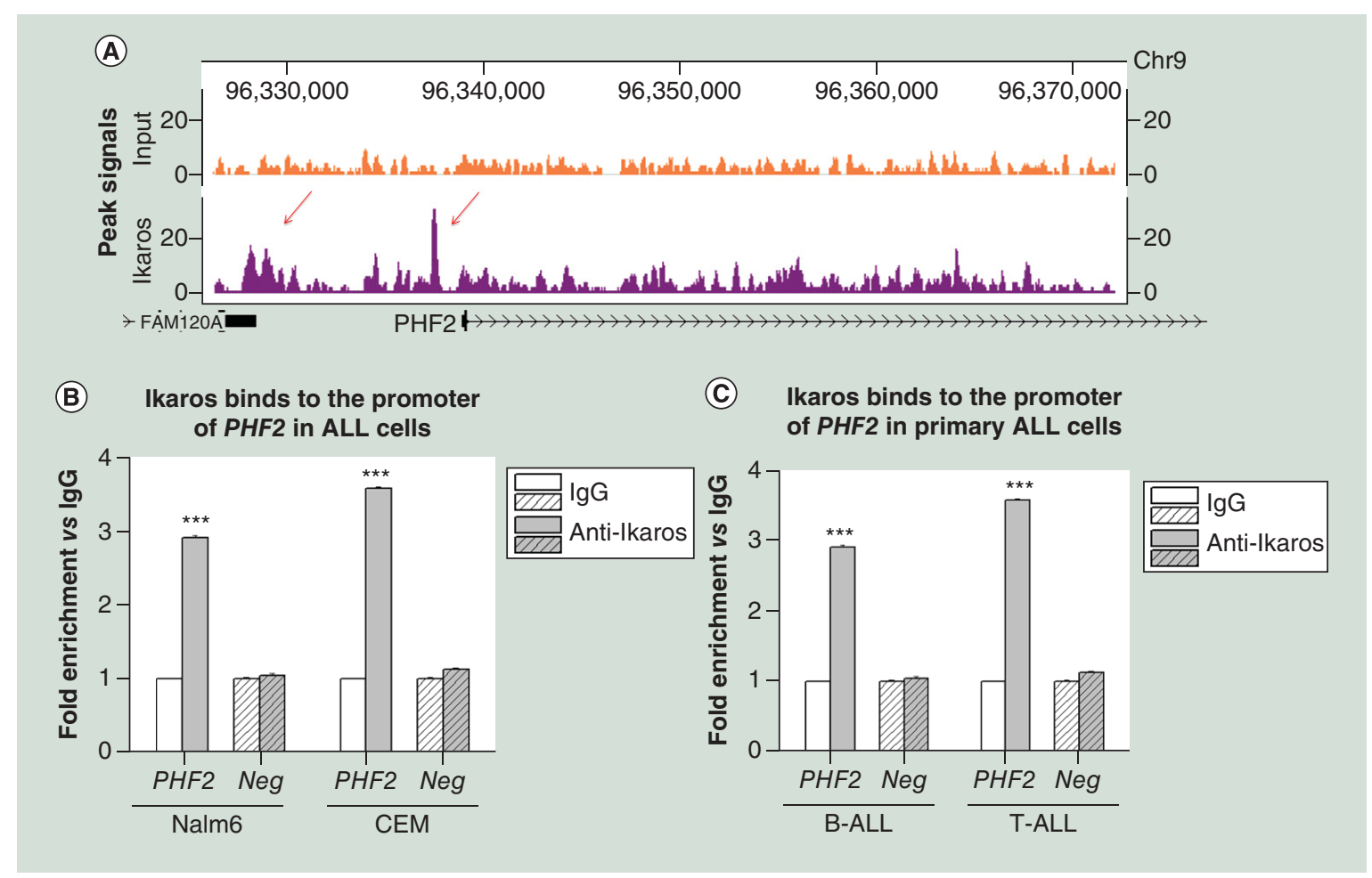

Figure 2. IKAROS binds to the promoter of PHF2 in Nalm6 B-cell acute lymphoblastic leukemia cells identified by ChIP-seq. (A) IKAROS-binding peaks on PHF2 promoter. The ChIP-seq data (GSE44218) was our previously published [18]; (B \& C) quantitative ChIP data show the IKAROS bindings to the promoter of PHF2 in ALL cell lines (B) and primary patients' samples (C).

ChIP: Chromatin immunoprecipitation; Neg: IKAROS-negative-binding control primers.

higher percentage of delayed remission $>4$ weeks ( 48.7 vs $19.0 \%$; $\mathrm{p}=0.015$ ) compared with the cohort with high PHF2 expression (Table 1).

We also analyzed the correlation of PHF2 expression with survival and found no significant difference in the median OS between the patients with PHF2 low and high expression (Supplementary Figure 2). While not statistically significant, we did note a trend toward a shorter median RFS in B-ALL patients with PHF2 low expression compared with the cohort with high PHF2 expression (9 months [5.581,12.419 months] vs 12 months [8.545,15.455 months]; $\mathrm{p}=0.139$ ) (Supplementary Figure 2).

IKAROS binds to the PHF2 promoter \& regulates its expression in ALL

To address the potential mechanism underlying low PHF2 expression we analyzed transcription factor motifs in the PHF2 promoter region. ChIP-seq data identified IKAROS-binding peaks in the PHF2 promoter region in Nalm6 B-ALL (Figure 2A) and primary B-ALL cells (Supplementary Figure 3) [18,19]. IKAROS binding was confirmed by quantitative ChIP assay in ALL cell lines (Figure 2B) and primary ALL patient samples (Figure 2C). These data indicate a direct effect of IKAROS on PHF2 transcription. Consistent with these data, expression of IKAROS promotes PHF2 promoter activity by a luciferase reporter assay (Figure 3A) and increases PHF2 mRNA levels in Nalm6 and CEM cells (Figure 3B). Conversely, efficient IKAROS knockdown decreases PHF2 expression in Nalm6 and CEM cells (Figure 3C).

Correlation of IKZF1 deletion with PHF2 low expression in B-ALL patients

We next analyzed a possible correlation between IKZF1 levels with PHF2 in primary B-ALL cells [27-29]. We found IKZF1 mRNA levels were positively correlated with PHF2 expression in B-ALL (GSE11877) and T-ALL (GSE26713) cohorts (Supplementary Figure 4). We also found PHF2 expression was significantly lower in B-ALL cells with $I k 6(0.03201 \pm 0.000527$ vs $0.11331 \pm 0.05773 ; \mathrm{p}=0.033$; Figure $3 \mathrm{D})$. This is consistent with our 
(A) Ikaros regulate promoter activity of PHF2

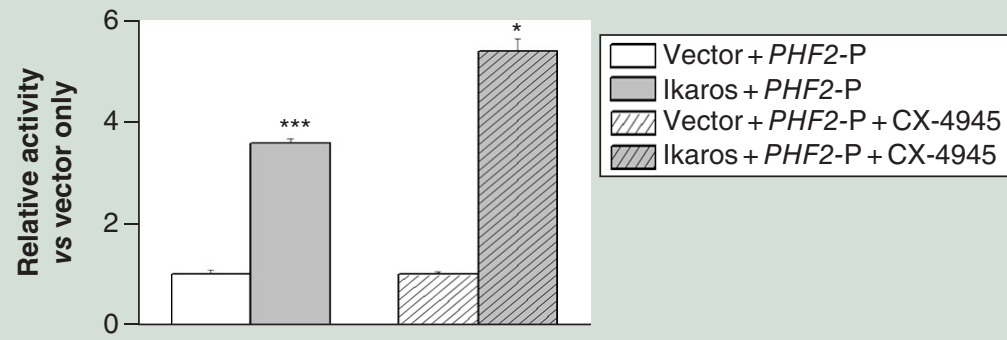

(B) Ikaros promotes PHF2 expression in ALL cells
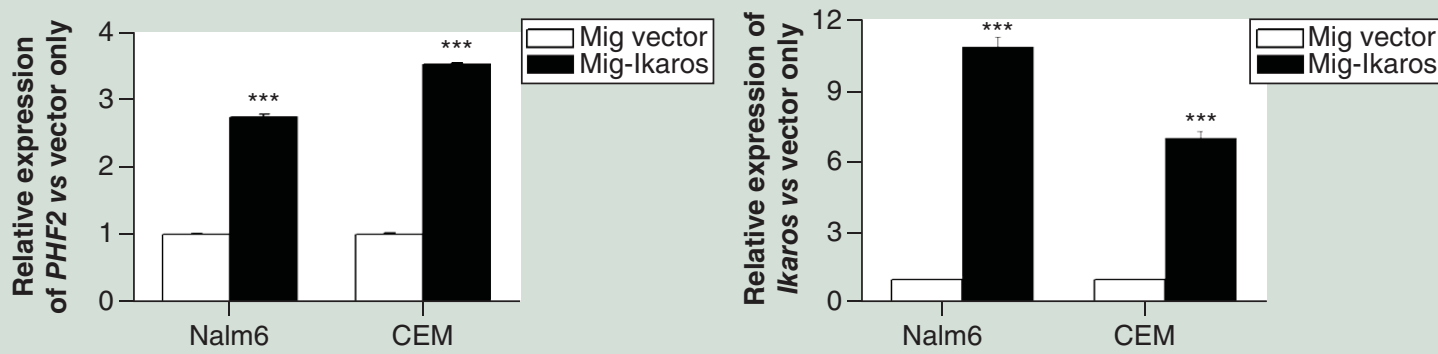

(C)

IKZF1 knockdown suppresses PHF2 transcription
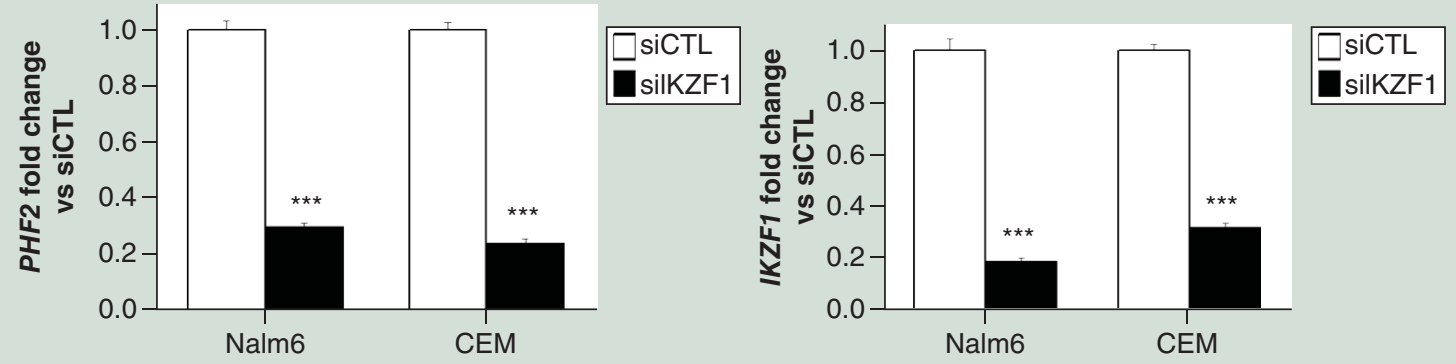

(D) IKZF1 deletion (Ik6+) associates PHF2 low expression in B-ALL patients $p=0.03332$

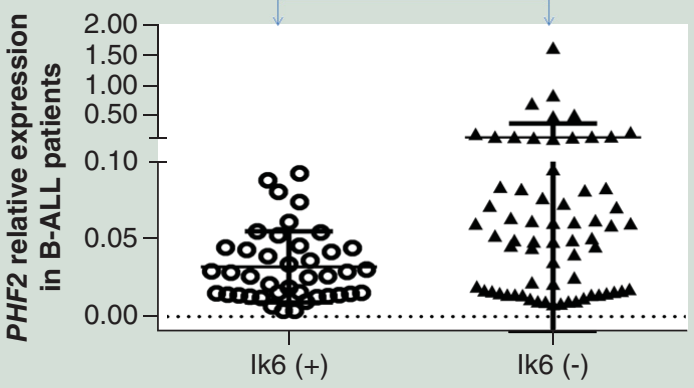

Figure 3. IKAROS promotes PHF2 expression in acute lymphoblastic leukemia.

(A) The activity of PHF2 promoters measured by luciferase reporter assay following transfection with IKAROS or control vector in HEK293 cells or that with CX-4945 treatment. ${ }^{* *} \mathrm{p}<0.001$ compared with pCDNA3.1 vector only; ${ }^{*} p<0.05$ compared with no CX-4945 treatment. (B) Expression of IKAROS promotes PHF2 expression in ALL cells. Compared with Mig vector: $* * * p<0.01$ compared with Mig vector only; (C) IKAROS knockdown by shRNA suppresses PHF2 expression in ALL cells. ${ }^{* *} \mathrm{p}<0.01$ compared with siCTL group. Data shown in A-C are representative of two to three independent experiments; (D) Comparison of PHF2 expression in the patients with Ik6(+) versus that of Ik6(-) in B-ALL.

ALL: Acute lymphoblastic leukemia; B-ALL: B-cell acute lymphoblastic leukemia; siCTL: Scramble shRNA control. 

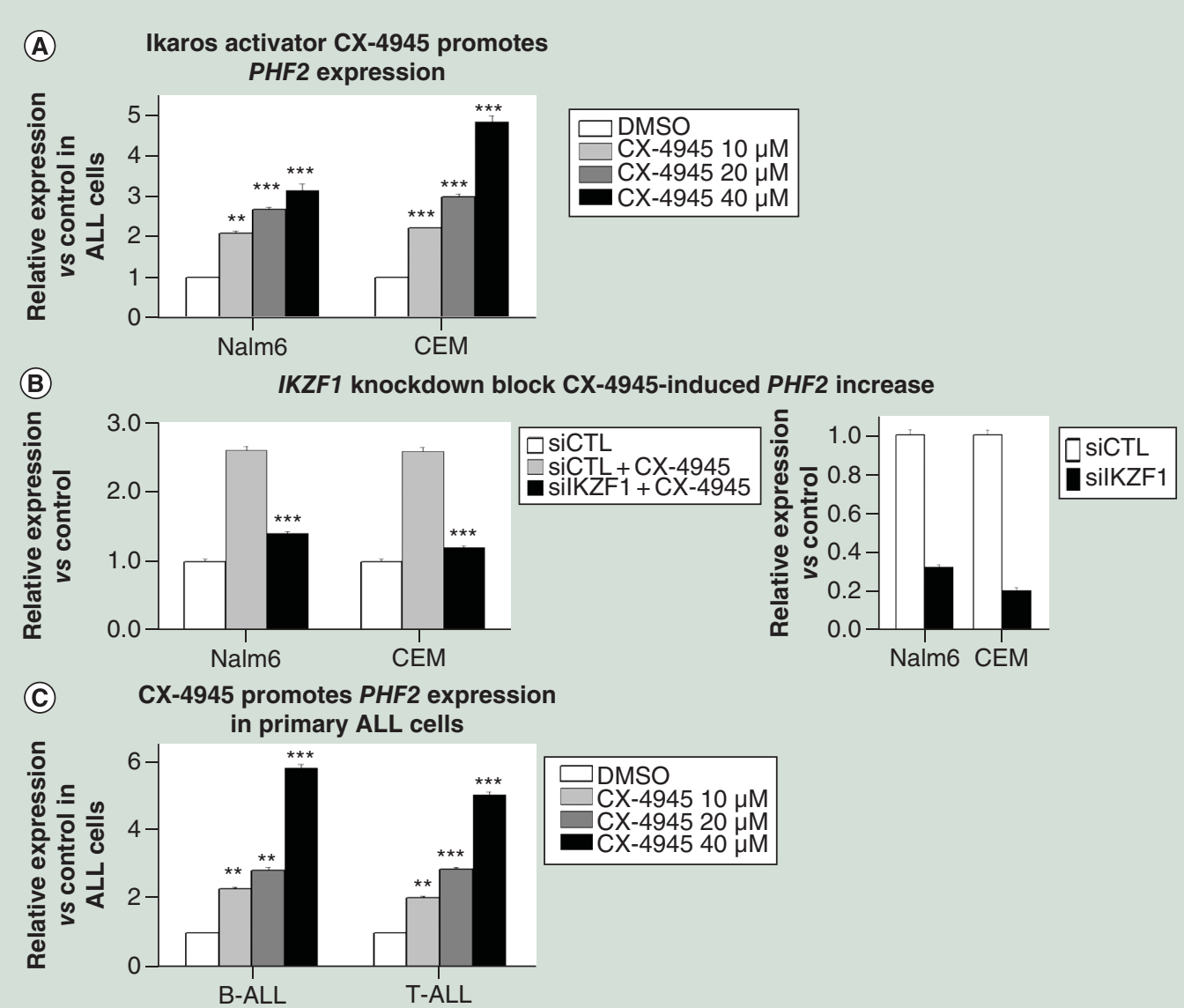

Figure 4. CX-4945 promotes PHF2 expression in an IKAROS-dependent manner.

(A) CX-4945 treatment induced increase of PHF2 expression in Nalm6 and CEM cells; (B) effect of IKAROS knockdown by shRNA on CX-4945-induced suppression of PHF2 expression. $* * * p<0.01$ compared with siCTL group; (C) CX-4945 promotes PHF2 expression in primary cells from ALL patients. Data shown in A-C are representative of two to three independent experiments.

ALL: Acute lymphoblastic leukemia; siCTL: Scramble shRNA control.

finding that B-ALL patients with PHF2 low expression have a significantly higher percentage of $I k 6$ (Table 1). These data suggest that a IKZF1 deletion may contribute to low PHF2 expression in B-ALL patients.

\section{CK2 inhibitor CX-4945 promotes PHF2 expression by enhancing IKAROS activity}

CX-4945 treatment facilitates the effect of IKAROS-mediated increase of PHF2 promotor activity (Figure 3A). Through qPCR, we also showed that treatment of Nalm6 and CEM cells with CX-4945 promoted expression of PHF2 mRNA in a dose-dependent manner (Figure 4A), and that IKZF1 knockdown significantly blocked this effect (Figure 4B). The effect of CX-4945 on PHF2 mRNA levels was also observed in primary B- and T-ALL cells (Figure 4C). Our results indicate that IKAROS not only binds to the PHF2 promoter but that treatment with CX-4945 enhances IKAROS tumor suppressor activity, promoting PHF2 expression in leukemia cells.

\section{Enhanced IKAROS activity by CK2 inhibition increases H3K4me3 occupancy at the PHF2 promoter}

Epigenetic regulation is critically important in mediating precise gene expression. We reported IKAROS regulates gene expression through epigenetic regulation [18,19]. Here, we also explored the epigenetic mechanism by which IKAROS regulates PHF2 expression. We performed ChIP assay for IKAROS, and HDAC1 and Mi2, the two key components of NuRD complex, and amplified the resulting PHF2 promoter sequences. No significant HDAC1 and Mi2 bindings are observed in the PHF2 promoter in both Nalm6 and CEM cells (Supplementary Figure 5A \& B). CX-4945 treatment significantly increases the binding of IKAROS to the PHF2 promoter region compared with 
(A)

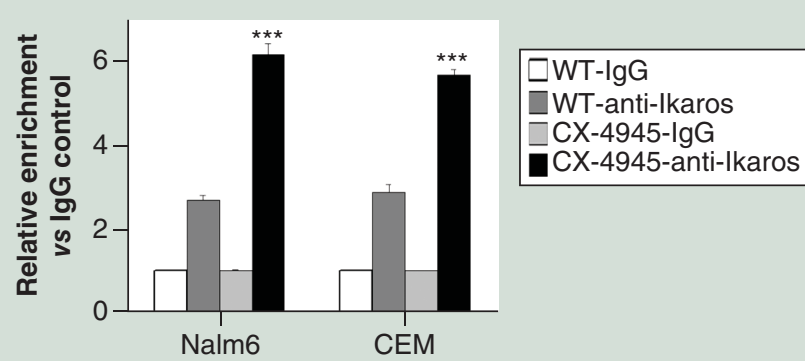

(C)

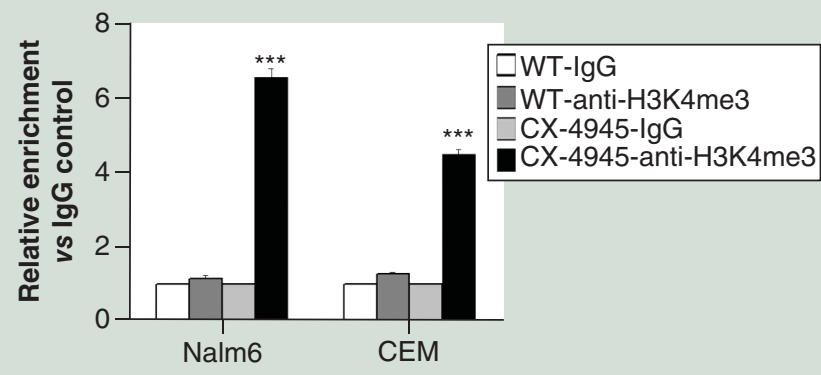

(B)

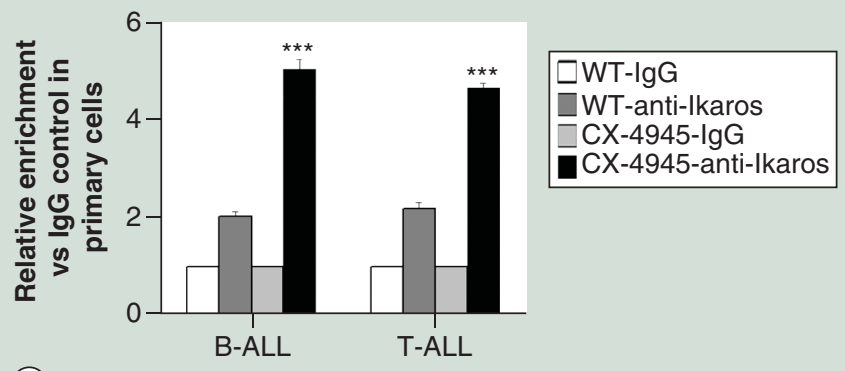

(D)

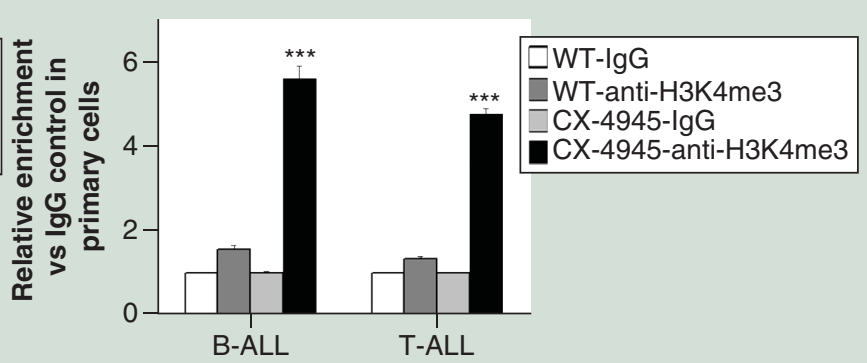

Figure 5. Chromatin switches upon CX-4945 treatment. (A-C) CX-4945 increases the enrichment of IKAROS (A, B) and H3K4me3 (C \& D) in promoter of PHF2 in ALL cell lines (A \& C) and primary cells (B \& D).

Data shown are representative of three independent experiments.

untreated Nalm6 B-ALL and CEM T-ALL cells (Figure 5A), as well as primary B-ALL and T-ALL cells (Figure 5B). However, CX-4945 treatment shows no effect of HDAC1 and Mi2 binding on the promoter in Nalm6 and CEM cells (Supplementary Figure 5A \& B). Instead, CX-4945 treatment increased H3K4me3 occupancy at the PHF2 promoter region in Nalm6 and CEM cells (Figure 5C) and in primary B- and T-ALL cells (Figure 5D), indicating the IKAROS activate the PHF2 expression by forming an open chromatin with H3k4me3.

\section{Discussion}

The discovery of histone demethylases has provided us with new insights into understanding the epigenetic landscape of the chromatin environment of cancer cells. The aberrant expression of histone demethylases in human cancers can potentially provide additional therapeutic targets in the treatment of cancer [30]. Prior studies have demonstrated that PHF2 is H3K9 demethylase, which subsequently participates in the epigenetic regulation of transcription [1].

H3K9 modification provides a universal mechanism of epigenetic gene regulation, thus PHF2 is suspected to be involved in a diverse set of biological functions including as a tumor suppressor. Here, our data indicate that PHF2 is downregulated in ALL and that PHF2 low expression is associated with leukemia cell proliferation and several poor prognostic markers. These data also reveal the oncogenic effect of PHF2 low expression in ALL. Our data are consistent with previous reports which indicate that PHF2 low expression is associated with poor outcomes in human cancer $[1-2,4-5,7,16]$.

Unlike most other histone demethylases, which are overexpressed in human cancers [13,31-33], PHF2 is deleted or hypermethylated in its promoter region in breast cancer and downregulated in head and neck cancers $[2,3]$. In addition, other reports confirm PHF2 is downregulated in colon cancer and involved in the epigenetic regulation of 553 [7]. These reports indicate that PHF2 low expression is involved in oncogenesis. Our data demonstrated that PHF2 is downregulated in adult ALL patients compared with normal controls; however, the downstream targets of PHF2 in the oncogenesis of ALL still need further clarification.

There have been few reports concerning the regulation of histone demethylase expression in human cancers. Histone demethylase 5B (KDM5B) is reported to be transcriptionally suppressed by IKAROS, HDAC1 and CK2 in ALL [13]. Here, we found that IKAROS directly promotes PHF2 transcription in ALL, and that an IKZF1 
gene deletion is associated with low PHF2 expression in high-risk ALL. Interestingly, we found restoring IKAROS function by CK2 inhibition can dramatically increase the H3K4me3 enrichment in the promoter of PHF2 in ALL cells. Thus, our data suggest that IKAROS promotes PHF2 expression by forming active chromatin. These data not only suggested the oncogenic roles of IKAROS dysfunction-induced PHF2 low expression, but also indicated that restoring IKAROS function is important to restoring the altered epigenomic state of IKAROS targets.

\section{Conclusion}

This is the first time to demonstrate that low PHF2 expression is significantly associated with acute lymphoblastic leukemia. Indeed, we found that PHF2 low expression was significantly associated with leukemia cell proliferation and several poor prognostic indicators in adult ALL patients. Moreover, we discovered that IKAROS directly regulates PHF2 transcription, and that CK2 inhibition, by restoring IKAROS function, promotes PHF2 transcription through histone modification. Our data, therefore, demonstrate the oncogenic effects of IKAROS dysfunction induce decreases in PHF2 expression. This also suggests that this dysfunction may represent a possible underlying mechanism in high-risk ALL. We suggest that PHF2 expression levels may be integrated into future adult ALL risk-stratification models.

\section{Summary points}

- PHF2 expression is significantly reduced in subsets of acute lymphoblastic leukemia (ALL) patients.

- PHF2 low expression correlates with leukemia cell proliferation and poor prognostic markers in B-cell ALL.

- Patients with IKAROS deletion have significantly lower PHF2 expression.

- IKAROSs directly promote PHF2 expression.

- Casein kinase II inhibitor significantly promotes PHF2 expression in an IKAROS-dependent manner through histone modification.

- PHF2 low expression works with the IKAROS gene deletion drive oncogenesis of ALL.

- Promotion of PHF2 expression may be one of the mechanisms underlying therapeutic effect of Ck2 inhibitor on high-risk ALL.

Acknowledgements

The authors thank J Koster, University of Amsterdam, the Netherlands for approval of use of microarray data on 'R2: Genomics Analysis and Visualization Platform' (http://r2.amc.nl).

Availability of data \& materials

The datasets generated from the patients during the current study are not publicly available in accordance with local health research ethics protocols but may be available from the corresponding author.

Authors' contribution

Z Ge, Y Gu, Q Han, Q Ge, G Gao, J Ma, H Song, J Hu, B Chen, S Dovat, C Song performed experiments and analyzed data; C Song, Z Ge, S Dovat designed and supervised data analysis; C Song, Z Ge, J Sloane, G Gao wrote the manuscript.

Financial \& competing interests disclosure

This work is supported in part by Milstein Medical Asian American Partnership (MMAAP) Foundation Research Project Award in Hematology (2017); The National Natural Science Foundation of China (81770172, 81270613); The Key Research \& Technology Projects in Jiangsu Province (BE2017747); The Fundamental Research Funds for the Central Universities (2242017K40271, 2242016K40143); Jiangsu Province Key Medical Talents (RC2011077); The Scientific Research Foundation for the Returned Overseas Chinese Scholars; State Education Ministry (39th); China Postdoctoral Science Foundation (20090461134); Special grade of the financial support from China Postdoctoral Science Foundation (201003598); The Six Great Talent Peak Plan of Jiangsu (2010-WS-024) (to Z Ge). This work has also been partially supported by NIH, National Cancer Institute grants (R01CA209829, R01CA213912), Hyundai Hope on Wheels Scholar Grant, the Four Diamonds Fund of the Pennsylvania State University College of Medicine (to S Dovat and C Song); Bear Necessities Pediatric Cancer Foundation, Alex's Lemonade Stand Foundation and the John Wawrynovic Leukemia Research Scholar Endowment (to S Dovat). The authors have no other relevant affiliations or financial involvement with any organization or entity with a financial interest in or financial conflict with the subject matter or materials discussed in the manuscript apart from those disclosed. 
No writing assistance was utilized in the production of this manuscript.

Ethical conduct of research

All the patients provided their written informed consent in accordance with the Declaration of Helsinki before enrollment in the study. The study was approved by the Institutional Review Board of the Nanjing Medical University and Zhongda Hospital Southeast University, Nanjing, China.

Supplementary data

To view the supplementary data that accompany this paper please visit the journal website at: www.futuremedicine.com/doi/full/10.2217/epi-2017-0092

\section{Reference}

1. Wen H, Li J, Song T, Lu M et al. Recognition of histone H3K4 trimethylation by the plant homeodomain of PHF2 modulates histone demethylation. J. Biol. Chem. 285(13), 9322-9326 (2010).

2. Sinha S, Singh RK, Alam N et al. Alterations in candidate genes PHF2, FANCC, PTCH1 and XPA at chromosomal 9q22.3 region: pathological significance in early- and late-onset breast carcinoma. Mol. Cancer 7, 84 (2008).

3. Ghosh A, Ghosh S, Maiti GP et al. Association of FANCC and PTCH1 with the development of early dysplastic lesions of the head and neck. Ann. Surg. Oncol. 19(Suppl. 3), S528-S538 (2012).

4. Baba A, Ohtake F, Okuno Y et al. PKA-dependent regulation of the histone lysine demethylase complex PHF2-ARID5B. Nat. Cell Biol. 13(6), 668-675 (2011).

5. Okuno Y, Ohtake F, Igarashi K et al. Epigenetic regulation of adipogenesis by PHF2 histone demethylase. Diabetes 62(5), 1426-1434 (2013).

6. Stender JD, Pascual G, Liu W et al. Control of proinflammatory gene programs by regulated trimethylation and demethylation of histone H4K20. Mol. Cell 48(1), 28-38 (2012).

7. Lee KH, Park JW, Sung HS et al. PHF2 histone demethylase acts as a tumor suppressor in association with p53 in cancer. Oncogene 34(22), 2897-2909 (2015).

8. Pattabiraman DR, Bierie B, Kober KI et al. Activation of PKA leads to mesenchymal-to-epithelial transition and loss of tumor-initiating ability. Science 351(6277), aad3680 (2016).

9. Cloos PA, Christensen J, Agger K et al. Erasing the methyl mark: histone demethylases at the center of cellular differentiation and disease. Genes Dev. 22(9), 1115-1140 (2008).

10. D'Oto A, Tian QW, Davidoff A M et al. Histone demethylases and their roles in cancer epigenetics. J. Med. Oncol. Ther. 1(2), 34-40 (2016).

11. Pfister SX, Ashworth A. Marked for death: targeting epigenetic changes in cancer. Nat. Rev. Drug Discov. 16(4), 241-263 (2017).

12. Ntziachristos P, Tsirigos A, Welstead GG et al. Contrasting roles of histone 3 lysine 27 demethylases in acute lymphoblastic leukaemia. Nature 514(7523), 513-517 (2014).

13. Wang H, Song C, Ding Y et al. Transcriptional regulation of JARID1B/KDM5B histone demethylase by IKAROS, histone deacetylase 1 (HDAC1), and casein kinase 2 (CK2) in B-cell acute lymphoblastic leukemia. J. Biol. Chem. 291(8), 4004-4018 (2016).

14. Ablain J, de The H. Retinoic acid signaling in cancer: the parable of acute promyelocytic leukemia. Int. J. Cancer 135(10), 2262-2272 (2014).

15. Wang Q, Ma S, Song N et al. Stabilization of histone demethylase PHF8 by USP7 promotes breast carcinogenesis. J. Clin. Invest. 126(6), 2205-2220 (2016).

16. Lee C, Kim B, Song B et al. Implication of PHF2 expression in clear cell renal cell carcinoma. J. Pathol. Transl. Med. 51(4), 359-364 (2017).

17. Churchman ML, Mullighan CG. Ikaros: exploiting and targeting the hematopoietic stem cellniche in B-progenitor acute lymphoblastic leukemia. Exp. Hematol. 46, 1-8 (2017).

18. Song C, Gowda C, Pan X et al. Targeting casein kinase II restores IKAROS tumor suppressor activity and demonstrates therapeutic efficacy in high-risk leukemia. Blood 126(15), 1813-1822 (2015).

19. Song $\mathrm{C}$, Pan $\mathrm{X}, \mathrm{Ge} \mathrm{Z}$ et al. Epigenetic regulation of gene expression by IKAROS, HDAC1 and casein kinase II in leukemia. Leukemia 30(6), 1436-1440 (2016).

20. Ge Z, Guo X, Li J et al. Clinical significance of high c-MYC and low MYCBP2 expression and their association with IKAROS dysfunction in adult acute lymphoblastic leukemia. Oncotarget 6(39), 42300-42311 (2015).

21. Song C, Li Z, Erbe AK et al. Regulation of IKAROS function by casein kinase 2 and protein phosphatase 1. World J. Biol. Chem. 2(6), 126-131 (2011). 
22. Ge Z, Gu Y, Han Q et al. Targeting high dynamin-2 (DNM2) expression by restoring IKAROS function in acute lymphoblastic leukemia. Sci. Rep. 6, 38004 (2016).

23. Guo X, Zhang R, Liu J et al. Characterization of LEF1 high expression and novel mutations in adult acute lymphoblastic leukemia. PLoS ONE 10, e0125429 (2015).

24. Campana D, Janossy G, Bofill M et al. Human B cell development. I. Phenotypic differences of B lymphocytes in the bone marrow and peripheral lymphoid tissue. J. Immunol. 134(3), 1524-1530 (1985).

25. Popescu M, Gurel Z, Ronni T et al. IKAROS stability and pericentromeric localization are regulated by protein phosphatase 1. J. Biol. Chem. 284(20), 13869-13880 (2009).

26. Wang H, Song C, Gurel Z et al. Protein phosphatase 1 (PP1) and casein kinase II (CK2) regulate IKAROS-mediated repression of TdT in thymocytes and T-cell leukemia. Pediatr. Blood Cancer 61(12), 2230-2235 (2014).

27. Homminga I, Vuerhard MJ, Langerak AW et al. Characterization of a pediatric T-cell acute lymphoblastic leukemia patient with simultaneous LYL1 and LMO2 rearrangements. Haematologica 97(2), 258-261 (2012).

28. Kang H, Chen IM, Wilson CS et al. Gene expression classifiers for relapse-free survival and minimal residual disease improve risk classification and outcome prediction in pediatric B-precursor acute lymphoblastic leukemia. Blood 115(7), 1394-1405 (2010).

29. Harvey RC, Mullighan CG, Wang X et al. Identification of novel cluster groups in pediatric high-risk B-precursor acute lymphoblastic leukemia with gene expression profiling: correlation with genome-wide DNA copy number alterations, clinical characteristics, and outcome. Blood 116(23), 4874-4884 (2010).

30. Hojfeldt JW, Agger K, Helin K. Histone lysine demethylases as targets for anticancer therapy. Nat. Rev. Drug Discov. 12(12), 917-930 (2013).

31. Zhu N, Chen M, Eng R et al. MLL-AF9- and HOXA9-mediated acute myeloid leukemia stem cell self-renewal requires JMJD1C. J. Clin. Invest. 126(3), 997-1011 (2016).

32. Chen M, Zhu N, Liu X et al. JMJD1C is required for the survival of acute myeloid leukemia by functioning as a coactivator for key transcription factors. Genes Dev. 29(20), 2123-2139 (2015).

33. Jung H, Chae YC, Kim JY et al. Regulatory role of G9a and LSD1 in the transcription of olfactory receptors during leukaemia cell differentiation. Sci. Rep. 7, 46182 (2017). 
(

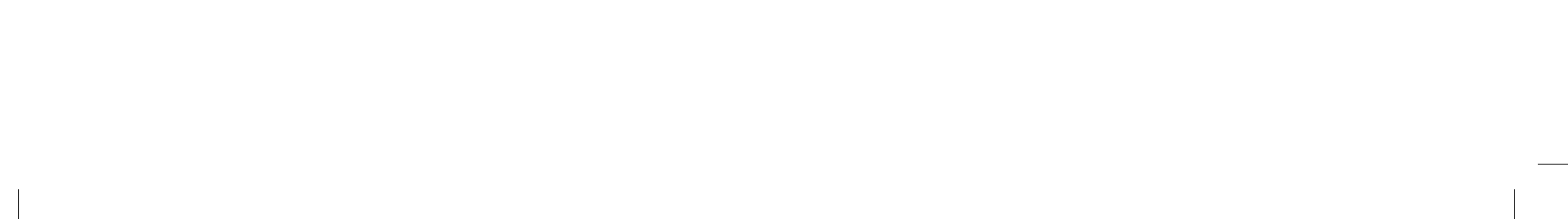




\title{
Vitamin $B_{12}$ supplementation influences methylation of genes associated with Type 2 diabetes and its intermediate traits
}

\author{
Dilip K Yadav ${ }^{1}$, Smeeta Shrestha ${ }^{1,2}$, Karen A Lillycrop ${ }^{3}$, Charu V Joglekar ${ }^{4}$, Hong Pan ${ }^{5}$, \\ Joanna D Holbrook ${ }^{5,6}$, Caroline HD Fall ${ }^{7}$, Chittaranjan S Yajnik ${ }^{\ddagger} 4$ \& Giriraj R Chandak ${ }^{*}, \ldots 1,8$ \\ ${ }^{1}$ Genomic Research on Complex Diseases (GRC Group), CSIR-Centre for Cellular \& Molecular Biology, Hyderabad, Telangana, 500 \\ 007, India \\ ${ }^{2}$ Building No 7, School of Basic \& Applied Sciences, Dayananda Sagar University, Shavige Malleshwara Hills, Kumaraswamy Layout, \\ Bangalore 560 078, Karnataka,India \\ ${ }^{3}$ Research Centre for Biological Sciences, Institute of Developmental Sciences, Southampton General Hospital, Southampton, \\ SO16 6 YD, UK \\ ${ }^{4}$ Diabetes Unit, King Edward Memorial Hospital \& Research Centre, Rasta Peth, Pune, Maharashtra, 411011 , India \\ ${ }^{5}$ Singapore Institute for Clinical Sciences, A*STAR, Brenner Centre for Molecular Medicine, 30 Medical Drive, 119521, Singapore \\ ${ }^{6}$ Human Development \& Health Academic Unit, University of Southampton \& National Institute for Health Research Southampton \\ Biomedical Research Centre, University of Southampton \& University Hospital Southampton NHS Foundation Trust, Tremona Road, \\ Southampton, SO16 6 YD, UK \\ ${ }^{7}$ MRC Lifecourse Epidemiology Unit, University of Southampton, Southampton General Hospital, Southampton, SO16 6 YD, UK \\ ${ }^{8}$ Adjunct Faculty, Human Genetics Unit, Genome Institute of Singapore, Biopolis, 138672, Singapore \\ * Author for correspondence: Tel.: +91 402719 2748; Fax: +91 402716 0591; chandakgrc@ccmb.res.in \\ $\ddagger$ Authors contributed equally
}

\begin{abstract}
Aim: To investigate the effect of $B_{12}$ and/or folic acid supplementation on genome-wide DNA methylation. Methods: We performed Infinium HumanMethylation450 BeadChip (Zymo Research, CA, USA) assay in children supplemented with $B_{12}$ and/or folic acid ( $n=12$ in each group) and investigated the functional mechanism of selected differentially methylated loci. Results: We noted significant methylation changes postsupplementation in $\mathrm{B}_{12}$ (589 differentially methylated $\mathrm{CpGs}$ and 2892 regions) and $\mathrm{B}_{12}+$ folic acid (169 differentially methylated CpGs and 3241 regions) groups. Type 2 diabetes-associated genes TCF7L2 and FTO; and a miRNA, miR21 were further investigated in another $\mathrm{B}_{12}$-supplementation cohort. We also demonstrate that methylation influences miR21 expression and FTO, TCF7L2, CREBBP/CBP and SIRT1 are direct targets of miR21-3p. Conclusion: $\mathrm{B}_{12}$ supplementation influences regulation of several metabolically important Type 2 diabetes-associated genes through methylation of miR21. Hence, our study provides novel epigenetic explanation for the association between disordered one carbon metabolism and risk of adiposity, insulin resistance and diabetes and has translational potential.
\end{abstract}

First draft submitted: 20 August 2017; Accepted for publication: 20 October 2017; Published online: 14 November 2017

Keywords: DNA methylation $\bullet$ folic acid $\bullet$ miRNAs $\bullet$ molecular mechanisms $\bullet$ supplementation $\bullet$ Type 2 diabetes - vitamin $\mathrm{B}_{12}$

Vitamin $\mathrm{B}_{12}\left(\mathrm{~B}_{12}\right)$ is an essential dietary micronutrient for human metabolism. $\mathrm{B}_{12}$ deficiency is classically described in pernicious anemia [1] and is associated with neurological damage [2]. $\mathrm{B}_{12}$ deficiency also manifests as hyperhomocysteinemia, which is an important risk marker for cardiovascular disease [3], obesity related complexities [4], Type 2 diabetes mellitus (T2D) [5] and metabolic syndrome [6]. Vitamin $\mathrm{B}_{12}$ and folic acid play important roles in one-carbon metabolism (OCM); $\mathrm{B}_{12}$ functions as an essential coenzyme for methionine synthase, which catalyzes methylation of homocysteine to methionine in the presence of the folic acid metabolite, 5-methyl tetrahydrofolate. This is an important step in generating S-adenosyl methionine (SAM), the universal methyl donor in OCM, which plays an important role in transmethylation reactions and epigenetic regulation.

$\mathrm{B}_{12}$ deficiency is common in Indians and is mainly attributed to vegetarian diets, but folate deficiency is relatively rare [7]. We have demonstrated associations between maternal plasma $\mathrm{B}_{12}$, folate and homocysteine concentrations 
and fetal growth [8], and childhood neurocognitive function [9], adiposity and insulin resistance [10,11]. A Mendelian randomization analysis using a maternal methylene tetrahydrofolate reductase C677T variant, suggested a causal role for maternal homocysteine concentrations in influencing fetal growth [8]. Given the potential public health importance of these findings for fetal growth and programming of noncommunicable disorders, we performed a pilot trial of oral $\mathrm{B}_{12}$ and folic acid supplementation in children and adults (registration number: ISRCTN59289820) which demonstrated a significant lowering of homocysteine concentrations with physiological doses of $\mathrm{B}_{12}$ supplementation but not with folic acid alone [12]. In the present study, we investigated molecular changes associated with $\mathrm{B}_{12}$ and folic acid supplementation by comparing genome-wide DNA methylation changes in the children in this trial. We identified differential methylation of several genes associated with T2D and related intermediate traits in the groups which received $B_{12}$ supplementation either alone or with folic acid. We further demonstrate that $B_{12}$ supplementation, through methylation of a specific miRNA, influences regulation of several T2D-associated and metabolically important genes.

\section{Subjects \& methods}

Characteristics of the study populations

Discovery cohort

The present study included children from the Pune Maternal Nutrition Study (PMNS), which was established to examine the relationship between maternal nutrition status, fetal growth and long-term outcomes in the children [13]. The study design of the $\mathrm{B}_{12}$ /folic acid intervention in the extended PMNS cohort has been described earlier [12]. In brief, 119 families (parents and children trios) from the extended PMNS were randomized in groups and supplemented daily for 12 months with $\mathrm{B}_{12}(10 \mu \mathrm{g})$ and/or folic acid $(200 \mu \mathrm{g})$, to investigate their effect on plasma homocysteine levels. We randomly selected 12 children (out of a total 15-17 children per group) from each of the four supplementation groups - placebo (B0F0), folic acid (B0F200), B $\mathrm{B}_{12}(\mathrm{~B} 10 \mathrm{~F} 0), \mathrm{B}_{12}+$ folic acid (B10F200), and compared their methylome before and after supplementation (Figure 1). Detailed physical and biochemical measurements $\left(\mathrm{B}_{12}\right.$, folate and homocysteine concentrations) and white blood cell counts at baseline and one-year follow-up were measured using standard techniques as described earlier [12]. Mean compliance in the study at 12 months was over $80 \%$. Informed consent was obtained from the parents and the Institutional Ethics Committee of the King Edward Memorial Hospital Research Centre approved the study following established guidelines for human research by Indian Council of Medical Research, Ministry of Health, Government of India.

\section{Replication cohort}

Selected hits identified from the discovery study were investigated in another $\mathrm{B}_{12}$ intervention trial in school children (Chikki trial). The three intervention groups received daily nutrient bars for a period of 120 days under direct observation, and compliance was $>95 \%$. The bars were fortified with nothing (placebo), $\mathrm{B}_{12}$ with multiple micronutrients $(\mathrm{MMN})$, or only $\mathrm{B}_{12}\left(\mathrm{~B}_{12}\right.$; Figure 1$)$. Hemoglobin, white blood cell counts, plasma $\mathrm{B}_{12}$, folate and homocysteine concentrations were measured at baseline and postintervention using standard methods. Out of 178 children screened, 14 were excluded because of a low $\mathrm{B}_{12}(\leq 100 \mathrm{pmol} / \mathrm{l})$ or low hemoglobin concentration ( $\mathrm{Hb}$ $<10 \mathrm{~g} / \mathrm{dl}$ ). The remaining 164 children (placebo $[\mathrm{n}=55], \mathrm{B}_{12}[\mathrm{n}=54]$ and $\mathrm{MMN}[\mathrm{n}=55]$ ) were randomized. Mean compliance was similar in all three groups (94.5\%). The detailed composition of the nutrient bar is given in Supplementary Table 1 .

\section{DNA methylation studies}

DNA methylation profiling, processing \& quality control analysis

Genomic DNA was isolated from the blood using QIAmp DNA blood midi kit (Qiagen, Hilden, Germany) and $500 \mathrm{ng}$ was bisulfite converted using EZ-96 DNA Methylation-Gold Kit (Zymo Research, CA, USA) according to the manufacturer's instructions. The Infinium HumanMethylation450 Beadchip Array (Illumina, CA, USA) was used for generation of methylation profiles as per the manufactures' protocols. The arrays were scanned on an Illumina iScan scanner and all quality control probes were analyzed from the control dashboard using Illumina’s Genome Studio (v2011.1) methylation module (v1.9.0) with default settings and HumanMethylation450_15017482_v1.1 manifest file. The .idat files obtained from the iScan were imported into the R environment (version 3.3.0) and preprocessed using minfi package [14]. The methylation value $(\beta)$ represents the proportion of methylation and is calculated as the ratio between methylated probe intensity and total probe intensity (range: $0-1$ ). The normalization was done using the 'funnorm' [15] with noob background correction. Normalized methylation $\beta$ values were con- 


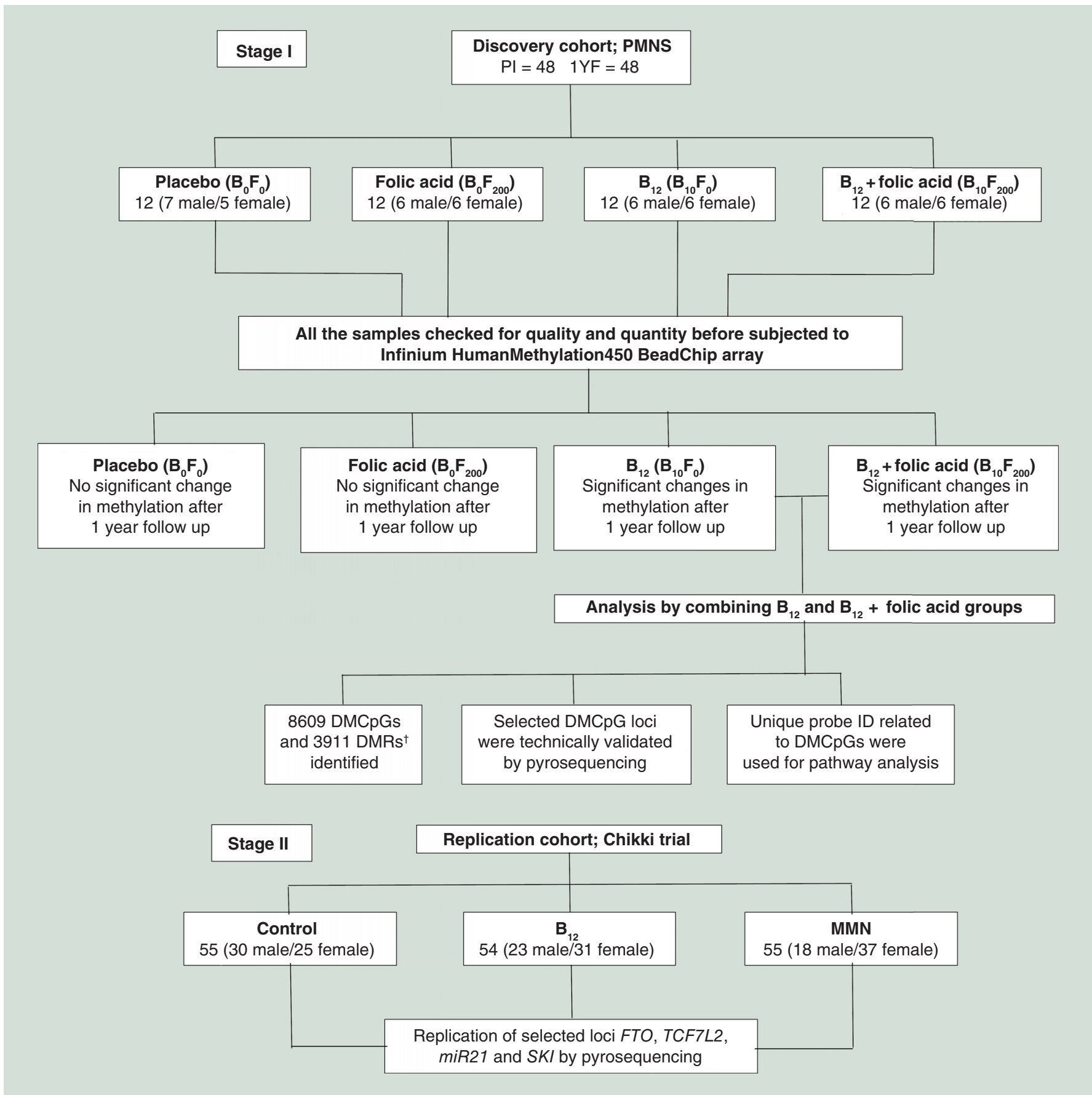

Figure 1. Overview of the study. Stage $I-B_{0} F_{0} /$ Placebo: No intervention; $B_{0} F_{200}$ : No $B_{12}$, folic acid $200 \mu g ; B_{10} F_{0}$ : $B_{12} 10 \mu g$, no folic acid; $B_{10} F_{200}: B_{12} 10 \mu \mathrm{g}$, folic acid $200 \mu \mathrm{g}$. Stage II - Control: No intervention; MMN: Multiple micronutrients $\left(B_{12} 1.8 \mu \mathrm{g}\right.$, folic acid $\left.300 \mu \mathrm{g}\right) ; B_{12}$ : $\mathrm{B}_{12} 2 \mu \mathrm{g}$; DMCpG: Differentially methylated CpG; DMR: Differentially methylated region.

$\dagger$ for DMR analysis FDR $\leq 0.02$ and mean methylation difference $\geq 2 \%$ was used.

1 YF: One year follow-up; FDR: False discovery rate; PI: Pre-intervention; PMNS: Pune Maternal Nutrition Study.

verted to $\mathrm{M}$ values which were used for downstream analyses. Probes on the $\mathrm{X}, \mathrm{Y}$ chromosomes and cross-reactive probes [16] were removed from the analysis. At last, a total of 458,057 probes' data from 94 subjects were carried forward for differentially methylated $\mathrm{CPG}(\mathrm{DMCpG})$ and region (DMR) analysis. 


\section{Detection of differentially methylated sites}

To identify supplementation-mediated DMCpGs, we tested methylation levels at baseline (pre) against those after 1 year follow-up (post) using linear regression. The regression analysis and empirical Bayes approach were performed using linear models for microarray data (limma) and the subjects as covariates, in order to account for the paired design [17]. In view of cellular heterogeneity [18] and strong collinearity in blood cell counts measured using variance inflation factor [19], principal components (PCs) were derived for the empirical cell counts and these were used in the linear model for adjustment. For genome-wide significance, we set the threshold for false discovery rate (FDR) adjusted to $\mathrm{p}<0.02$.

\section{Detection of dfferentially methylated regions}

We used the DMRcate Bioconductor R package [18] for identification of supplementation-mediated regional methylation differences. Briefly, DMRcate functions to calculate test statistics for each CpG probe using the limma empirical Bayes t-moderated statistic as mentioned in DMCpG analysis. DMRcate further re-calculates p-values at individual CpGs after modeling the Gaussian kernel smoothing using the Satterthwaite method50 within a predefined bandwidth of $\lambda=1000 \mathrm{bp}$ and scaling factor $\mathrm{C}=2$. The computed $\mathrm{p}$-values were adjusted for multiple testing using a Benjamin-Hochberg (BH) FDR threshold of 0.05 and the combined information from nearby significant $\mathrm{CpG}$ s within the bandwidth. DMRs were constructed by grouping FDR significant sites, which lie at maximum of $1000 \mathrm{bp}$ from each other and contain at least two or more CpGs. minFDR (minimum BH adjusted value) within a DMR is representative of the statistical inference for that region and the mean fold change (meanbetafc) is the mean $\beta$-fold change within the region. DMRcate analysis was performed to compare preand post-supplementation groups, where we defined significant DMRs having $\mathrm{BH}$ corrected $\mathrm{p}<0.2$, and for the combined $\mathrm{B}_{12}$ and non- $\mathrm{B}_{12}$ group analysis we defined significant DMRs having $\mathrm{BH}$ corrected $\mathrm{p}<0.05$. At last, on obtaining the DMRs for each analysis, we termed those DMRs significant which contained minFDR $<0.02$ and a meanbetafc of $2 \%$ in the final results output.

\section{Pyrosequencing \& genotyping}

Selected hits from the Infinium HumanMethylation450 BeadChip Array data were technically validated on the same individuals used in discovery experiment $(\mathrm{n}=12)$, but freshly bisulfite converted DNA samples were used. Similar approach was used for the replication analysis on individuals in the Chikki Trial $(\mathrm{n}=54-55$ in each group; Figure 1). Primers and assay files were designed using PyroMark Assay Design Software 2.0 (Qiagen; Supplementary Table 2). Pyrosequencing was performed using PyroMark ID 24 according to the manufacturer's instructions and the data were analyzed with the PyroMark Q24 software program (Qiagen).

\section{Bioinformatics analysis}

Genome coordinates provided by Illumina were used to annotate the significantly enriched DMCpGs and DMRs. Probes lacking an annotated gene identity and duplicate gene entries were removed and annotated genes (as per official UCSC reference), CpG islands, enhancers and promoter regions were analyzed further. We performed an enrichment analysis to examine whether the significant DMCpGs (FDR $<0.02$; absolute beta difference $>0.05$ ) were over- or under-represented in different biological features from the Infinium HumanMethylation 450 BeadChip annotation file. Annotated probes were tested for enrichment of DMCpGs using a two-tailed Fisher's exact test, compared with the frequency of DMCpGs in all annotated probes on the Infinium HumanMethylation 450 BeadChip array and on the DMCpGs obtained from the $\mathrm{B}_{12}$ and the $\mathrm{B}_{12}+$ folic acid groups.

The Ingenuity pathway analysis (IPA) software (Ingenuity Systems, Inc, CA, USA) contains probe identification information of the Infinium HumanMethylation450 BeadChip. The unique probe identifiers for DMCpGs obtained from each of the intervention groups (FDR $<0.02$; beta difference $<0.05$ ) were set up as 'Probe List' and were uploaded into the IPA. We used the 'Core Analysis' module to identify the canonical pathways and biological networks altered/regulated due to intervention in the groups studied and reported -10 logarithms of Fisher's exact test $\mathrm{p}$-values in canonical pathway analysis by IPA. Biological functions which were significant at Fischer's exact test were then assigned to network by determining a $\mathrm{p}$-value for the enrichment of the genes in the network for such functions compared with the whole Ingenuity Pathway Knowledge Base as a reference set. Online databases and miRNA target prediction tools such as miRDB [20], miRanda [21] and DIANA-microT [22] were used to identify the potential targets of $m i R 21$. 
Functional studies

Generation of constructs related to miR21 differentially methylated region

The miR21 promoter (miR21-Pro-pGL3B, 934 bp) and DMR (miR21-DMR-pGL3B, 256 bp) constructs were generated by cloning them in pGL3 basic vector (pGL3B; Promega, WI, USA) and sequences were verified by Sanger sequencing. Methylated miR21 DMR was generated by in vitro methylation of the DMR with CpG methyl transferase M.SssI in the presence of $32 \mathrm{mM} \mathrm{SAM}$ for $48 \mathrm{~h}$ at $37^{\circ} \mathrm{C}$. Mock methylation was performed in similar way but without M.SssI methyltransferase. Methylation status was verified by digesting the methylated and mock methylated DMR with methylation sensitive BceA1 enzyme (NEB, MA, USA). Methylated and mock methylated DMRs were re-ligated in pGL3B by incubating them for $16 \mathrm{~h}$ at $16^{\circ} \mathrm{C}$. The ligated products were gel purified and quantified before transfection in various cell lines. All primers are listed in Supplementary Table 3.

\section{Generation of the reporter constructs for validation of miR21-3p targets}

We generated reporter constructs of the predicted targets of miR21-3p (target-psiCHECK) by cloning the $500 \mathrm{bp}$ region of 3 '-UTR containing the potential seed sequences into psiCHECKTM-2 dual luciferase reporter vector (Promega) downstream to hRluc gene. For overexpressing miR21-3p (miR21-3p-pmU6), we adopted a method in which $21 \mathrm{bp}$ miRNA sequence was synthesized in a stem loop backbone oligo of $60 \mathrm{bp}$. The forward and reverse strands were synthesized in such way that on annealing, Bbs1 and Xba1 restriction sites were generated. The hybridized oligos were cloned into pmU6 vector having U6 promoter [23]. The same method was used to generate a control for miR21-3p (control-pmU6). We mutated seed sequences of target constructs by site-directed mutagenesis where the target constructs were amplified using primers containing the mutation and $2 \mathrm{X}$ Trans Taq High Fidelity (HiFi) PCR SuperMix according to manufacturers' protocols (Transgen Biotech, Beijing, China). After PCR amplification, templates were digested with $\mathrm{Dpn} 1$ at $37^{\circ} \mathrm{C}$ for $45 \mathrm{~min}$ and $5 \mu \mathrm{l}$ of Dpn1-digested amplicons were used for transformation. Sanger sequencing was used to confirm the mutations in the seed sequences (Supplementary Table 3).

\section{Dual luciferase reporter assay}

We performed dual luciferase reporter assay to evaluate the promoter activity of the miR21 DMR and the effect of methylation on promoter activity. We used three cell lines, HEK293, HepG2 and MIN6 cell lines and seeded individual cell lines at a density of $5.0 \times 10^{4}$ cells per well in 24-well plates, $24 \mathrm{~h}$ before transfection. Cells were co-transfected with different constructs (100 ng of each miR21DMR-pGL3B or miR21Pro-pGL3B constructs and $400 \mathrm{ng}$ of methylated or mock methylated construct per well) along with 1 ng of Renilla Luciferase (pRL) control vector using lipofectamine 2000 (Invitrogen, CA, USA). Cell lyses and dual luciferase assays were performed 24 $\mathrm{h}$ after transfection using dual luciferase reporter assay kit (Promega) on the Perkin Elmer multimode plate reader according to the manufacturer's instructions. Firefly luciferase activity was normalized by Renilla luciferase activity. For validation of $m i R 21$ - $3 p$ targets, each target-psiCHECK construct (100 ng), and either $300 \mathrm{ng}$ of pmU6-miR21$3 p$ or pmU6-control were co-transfected in the cell line. Luciferase assay was performed as described above except that on this occasion, the Renilla luciferase activity was normalized relative to the firefly luciferase activity.

\section{Electrophoretic mobility shift assay}

Methylated and mock methylated miR21 DMRs were radiolabeled using protocols described by the manufacturer (NEB). In brief, an equal amount $(1.5 \mu \mathrm{g})$ each of methylated and mock methylated miR21 DMR were incubated with $32 \mathrm{P}$ ATP and $10 \mathrm{U}$ polynucleotide kinase in $70 \mathrm{mM}$ Tris-HCL, $10 \mathrm{mM} \mathrm{MgCl}_{2}$ and $5 \mathrm{mM}$ dithiothreitol at room temperature for $1 \mathrm{~h}$. The labeled probes were purified using a sepharose bead column and eluted in $100 \mu \mathrm{l}$ of TE. The radiolabeled methylated and mock methylated DMR probes were incubated with $1.5 \mu$ of HEK293 cell nuclear extract $(5 \mathrm{mg} / \mathrm{ml}), 0.5 \mu \mathrm{l}$ polydIdC $(1 \mathrm{mg} / \mathrm{ml}$, Sigma-Aldrich, MO, USA) and $0.5 \mu$ l yeast tRNA $(1 \mathrm{mg} / \mathrm{ml}$, Invitrogen) in $20 \mu \mathrm{l}$ binding buffer (HEPES $20 \mathrm{mM} \mathrm{pH} \mathrm{7.9,} \mathrm{KCl} 150 \mathrm{mM}$, EDTA $1 \mathrm{mM}$ and Ficoll $8 \%$ ) on ice for $10 \mathrm{~min}$. Subsequently, the binding mix was loaded on $6 \%$ native polyacrylamide gel and run at 75 $\mathrm{V}$ at $4^{\circ} \mathrm{C}$ for $12 \mathrm{~h}$. After the run was over, the gel was exposed to phospho-imager screen (GE Healthcare, IL, USA) for $6 \mathrm{~h}$ followed by scanning by Personal Molecular Imager (BioRad, CA, USA).

\section{Statistical analysis}

Biochemical data were analyzed using SPSS software (v 17.0; SPSS, Inc, IL, USA). Demographic and biochemical values were reported as median and interquartile range. To investigate the significance of change in the values, a 
nonparametric Mann-Whitney test was conducted for across group (placebo vs other groups) and a paired $t$-test for within group (baseline vs 1 year follow-up) comparisons. All statistical analysis for the Infinium HumanMethylation450 BeadChip Array data was performed using ' $R$ ' as stated above. Methylation data from pyrosequencing were extracted using the PyroMark Q24 software (v.2.0.6) and CpG sites that 'failed' at the PyroMark software were excluded from the analysis. Variance inflation factor and PCs were calculated using R scripts. All methylation data were then adjusted for age, gender and blood count using linear regression in ' $R$ '. The adjusted methylation values were generated using the Kobor method [24] and compared at baseline (pre) and after supplementation (post) using paired student $t$-tests using Graphpad prism (v6.0, GraphPad Software, Inc, CA, USA). Median methylation differences were analyzed with the Mann-Whitney $U$ test for between-group comparisons (placebo vs supplementation subjects). Additionally, to analyze gender specific methylation changes, methylation data were stratified based on gender and methylation values were compared between the genders as mentioned above. Data in graphs were shown as mean \pm standard error of mean (mean \pm SEM) and results were considered significant at $\mathrm{p}<0.05$. All luciferase assays were performed in triplicate and repeated at least thrice. The data were normalized with the co-transfected vector and unpaired student $t$-test was performed to evaluate the significance level $(\mathrm{p}<0.05)$.

\section{Results}

\section{Cohort characteristics}

Characteristics of the children in the PMNS (discovery cohort) at baseline and after intervention are presented in Table 1. This population has a low $\mathrm{B}_{12}$ but adequate folate status, and hyperhomocysteinemia is common. Supplementation for 12 months resulted in higher concentrations of $\mathrm{B}_{12}$ in the $\mathrm{B}_{12}(\mathrm{~B} 10 \mathrm{~F} 0)$ and the $\mathrm{B}_{12}+$ folic acid (B10F200) groups (by 139.5 and $130.5 \mathrm{pmol} / \mathrm{l}$, respectively; $\mathrm{p}<0.01$ and $\mathrm{p}<0.05$, respectively) but it remained unchanged in the folic acid (B0F200) group. Folate levels increased in both the $\mathrm{B}_{12}+$ folic acid and folic acid groups (by 5.4 and $16.4 \mathrm{nmol} / \mathrm{l}$, respectively; $\mathrm{p}<0.01$ both) but decreased in the $\mathrm{B}_{12}$ group (by $4.5 \mathrm{nmol} / \mathrm{l}$; $\mathrm{p}<0.05)$. Plasma homocysteine levels did not change in the folic acid alone group but showed a reduction in both the $\mathrm{B}_{12}$ and $\mathrm{B}_{12}+$ folic acid groups (by 3.1 and $2.7 \mu \mathrm{mol} / \mathrm{l}$; $\mathrm{p}<0.01$ both). Placebo group (B0F0) did not show any change in plasma $\mathrm{B}_{12}$ and folate concentrations but plasma homocysteine levels increased (by $2.5 \mu \mathrm{mol} / 1 ; \mathrm{p}<0.01$ ). In the Chikki Trial (replication cohort; Table 2), plasma $\mathrm{B}_{12}$ concentrations increased with supplementation (along with multiple micronutrients $[\mathrm{MMN}]$ and $\mathrm{B}_{12}$ alone $\left[\mathrm{B}_{12}\right]$ ) by 91.0 and $82.0 \mathrm{pmol} / \mathrm{l}$, respectively ( $\mathrm{p}<0.0001$ for both), while no significant change was seen in the placebo group. Plasma folate concentrations increased by $27.8 \mathrm{nmol} / 1(\mathrm{p}<0.001)$ and $1.3 \mathrm{nmol} / \mathrm{l}(\mathrm{p}<0.05)$, respectively in the $M M N$ and $B_{12}$ groups, but remained unchanged in the placebo group. Both supplementation groups showed a reduction in plasma homocysteine concentrations ( 3.8 and $1.4 \mu \mathrm{mol} / \mathrm{l}$, respectively; $\mathrm{p}<0.0001$ ) but the levels increased in the placebo group $(1.9 \mu \mathrm{mol} / 1 ; \mathrm{p}<0.0001)$. A comparison of change in anthropometric measurements across the groups did not show any significant differences after supplementation indicating no additional effect of supplementation.

\section{Differentially methylated loci \& their biological relevance}

In the PMNS cohort, we compared methylation levels at approximately 483,000 loci measured on the Infinium HumanMethylation 450 BeadChip Array, pre- and post-supplementation in each group and across groups. Considering the small sample size, we used an FDR adjustment and a stringent FDR adjusted $\mathrm{p}<0.02$ for the analysis. At baseline, there were no significant differences in DNA methylation levels (FDR $<0.02$ and difference between group average $\%$ methylation levels $>5 \%$ ) among the four groups in the PMNS. On comparison of the pre- and post-supplementation methylation data, $12 \mathrm{DMCpGs}$ were detected in the placebo group, presumably representing background change over time and noise in the data. The folic acid group showed 19 DMCpGs, while the groups receiving $B_{12}$ alone and that with folic acid showed many more DMCpGs; 589 and 169 DMCpGs in the $B_{12}$ and $\mathrm{B}_{12}+$ folic acid groups, respectively (Figure 2A-D \& Table 3; Supplementary Tables 4 \& 5). On comparing the groups that received $\mathrm{B}_{12}\left(\mathrm{~B}_{12}\right.$ and $\mathrm{B}_{12}+$ folic acid groups) and those that did not (placebo and folic acid groups), we observed that the group which received $\mathrm{B}_{12}$ had 8609 DMCpGs while the group which did not receive $\mathrm{B}_{12}$ had only 519 significant DMCpGs at FDR $<0.02$ and beta difference $>5 \%$ (Table 3; Supplementary Tables $6 \& 7$ ). These observations clearly indicate that $\mathrm{B}_{12}$ supplementation has a larger impact than folic acid on the methylation status of various genes in this population. At last, we detected contiguous regions of differential methylation (DMR) containing multiple CpGs using the DMRcate algorithm ( $\operatorname{minFDR}<0.02$ and beta difference $>2 \%)$. We observed higher number of DMRs in the $\mathrm{B}_{12}+$ folic acid group compared with the $\mathrm{B}_{12}$ group (3241 vs 2891, respectively; Supplementary Tables 8 \& 9) while placebo and folic acid groups had only 18 and 27 DMRs, respectively (Table 3). 


\begin{tabular}{|c|c|c|c|c|}
\hline Groups & Placebo (B0F0) & Folic acid (BOF200) & $\mathrm{B}_{12}$ (B10F0) & $\mathrm{B}_{12}+$ folic acid (B10F200) \\
\hline \multicolumn{5}{|l|}{ Parameters } \\
\hline N (Male/female) & $12(7 \mathrm{M} / 5 \mathrm{~F})$ & $12(6 \mathrm{M} / 6 \mathrm{~F})$ & $12(6 \mathrm{M} / 6 \mathrm{~F})$ & $12(6 \mathrm{M} / 6 \mathrm{~F})$ \\
\hline \multicolumn{5}{|l|}{ Age (years) } \\
\hline Baseline & $9.1(8.9-9.2)$ & $9.0(8.8-9.2)$ & $8.9(8.7-9.1)$ & $8.9(8.9-9.2)$ \\
\hline Supplementation & $10.7(10.7-10.9)$ & $10.7(10.5-10.8)$ & $10.8(10.5-10.9)$ & $10.7(10.5-10.9)$ \\
\hline Change & $1.7(1.6-1.8)$ & $1.7(1.6-1.8)$ & $1.8(1.8-1.9)$ & $1.8(1.6-1.8)$ \\
\hline \multicolumn{5}{|l|}{ Height $(\mathrm{cm})$} \\
\hline Baseline & $124.9(123.2-127.1)$ & $127.8(122.6-133.5)$ & $126.5(122.5-128.5)$ & $126.3(119.8-129.4)$ \\
\hline Supplementation & $133.6(130.9-139.5)$ & $135.8(132.7-143.7)$ & $136.6(131.1-138.6)$ & $134.1(131.1-141.2)$ \\
\hline Change & $9.7(7.9-12.3)$ & $10.0(8.5-10.8)$ & $9.9(8.9-10.8)$ & $9.2(8.3-11.1)$ \\
\hline \multicolumn{5}{|l|}{ Weight (kg) } \\
\hline Baseline & $21.8(20.1-23.2)$ & $22.3(20.8-24.3)$ & $21.5(18.8-22.3)$ & $22.1(19.4-23.5)$ \\
\hline Supplementation & $24.4(23.6-30.1)$ & $26.6(24.8-30.2)$ & $25.6(24.1-27.3)$ & $25.2(24.3-28.7)$ \\
\hline Change & $4.1(3.2-4.5)$ & $4.8(3.3-5.4)$ & $4.6(3.5-6.0)$ & $4.3(2.8-6.1)$ \\
\hline \multicolumn{5}{|l|}{ BMI $\left(\mathrm{kg} / \mathrm{m}^{2}\right)$} \\
\hline Baseline & $13.9(13.2-14.5)$ & $13.8(12.8-14.8)$ & $13.1(12.7-14.5)$ & $13.9(13.4-14.2)$ \\
\hline Supplementation & $14.2(13.5-15.7)$ & $15.0(13.5-15.8)$ & $14.3(13.5-14.8)$ & $14.2(13.4-15.0)$ \\
\hline Change & $0.5(0.1-0.7)$ & $0.8(0.2-1.3)$ & $1.0(0.2-1.8)$ & $0.4(-0.1-1.2)$ \\
\hline \multicolumn{5}{|l|}{$\mathrm{B}_{12}(\mathrm{pmol} / \mathrm{l})$} \\
\hline Baseline & $217.0(157.0-269.0)$ & $193.5(161.8-301.8)$ & $177.0(137.5-196.5)$ & $155.0(117.0-231.8)$ \\
\hline Supplementation & $197.5(168.8-224.8)$ & $212.0(177.8-247.5)$ & $328.5(238.0-362.5)$ & $307.5(238.5-413.0)$ \\
\hline Change & $9.0(-40.0-33.0)$ & $-9.5(-49.0-46.0)$ & $139.5(68.3-209.8)^{\dagger * *}$ & $130.5(66.0-194.0)^{\dagger *}$ \\
\hline \multicolumn{5}{|l|}{ Folate $(\mathrm{nmol} / \mathrm{l})$} \\
\hline Baseline & $17.5(13.8-24.6)$ & $20.9(13.5-26.0)$ & $19.3(13.5-23.1)$ & $19.7(15.0-22.5)$ \\
\hline Supplementation & $21.0(15.9-23.1)$ & $41.0(26.7-49.1)$ & $13.8(10.9-17.2)$ & $25.7(17.8-30.9)$ \\
\hline Change & $1.6(-4.4-9.5)$ & $16.4(5.4-29.6)^{\dagger * *}$ & $-4.5(-7.7-0.4)^{\dagger *}$ & $5.4(0.1-12.6)^{\star *}$ \\
\hline \multicolumn{5}{|c|}{ Homocysteine $(\mu \mathrm{mol} / \mathrm{l})$} \\
\hline Baseline & $9.4(7.3-11.1)$ & $9.7(7.9-11.4)$ & $11.3(9.1-15.1)$ & $10.6(9.0-12.3)$ \\
\hline Supplementation & $12.6(9.8-16.0)$ & $9.8(8.1-15.4)$ & $7.6(6.3-9.2)$ & $7.8(6.1-9.0)$ \\
\hline Change & $2.5(0.9-4.6)^{\star *}$ & $0.5(-0.3-3.9)$ & $-3.1(-4.9-2.0)^{\dagger * *}$ & $-2.7(-4.5-1.6)^{\dagger} * *$ \\
\hline
\end{tabular}

Further, combining the data from both $\mathrm{B}_{12}$ supplementation groups identified more significant DMRs $\left(\mathrm{B}_{12}\right.$ and $\mathrm{B}_{12}+$ folic acid; $\mathrm{n}=3911$ ) in comparison to the two groups that did not receive $\mathrm{B}_{12}$ (placebo and folic acid, $\mathrm{n}=1725$; Table 3; Supplementary Tables $10 \& 11)$.

The majority of DMCpGs in the $\mathrm{B}_{12}(\mathrm{n}=432 / 589 ; 73.3 \%)$ and $\mathrm{B}_{12}+$ folic acid groups $(93 / 169 ; 55.03 \%)$ were hypomethylated. However, while the majority of DMRs in the $\mathrm{B}_{12}$ group ( $\mathrm{n}=1745 / 2908 ; 60.0 \%$ ) were hypomethylated, those in the $\mathrm{B}_{12}+$ folic acid group $(2331 / 3267 ; 71.35 \%)$ were mostly hypermethylated. The DMCpGs were unequally distributed with respect to the annotated genic features in both $\mathrm{B}_{12}$ and $\mathrm{B}_{12}+$ folic acid groups (Supplementary Table 12). The 589 DMCpG probes in the $\mathrm{B}_{12}$ group were located in 424 unique genes of which 75 were promoter-associated, 245 were enhancer-associated and 83 were in CpG islands. Similarly, in the $\mathrm{B}_{12}+$ folic acid group, $169 \mathrm{DMCpG}$ probes were distributed in 129 unique genes, 53 of which were in the promoter region, 50 and 61 were enhancer- and island-associated, respectively. Enrichment analysis demonstrated that DMCpGs were under-represented in TS1500, $1^{\text {st }}$ Exon, CpG island, S-shore, promoter and unclassified regulatory regions in the $\mathrm{B}_{12}$ group ( $\mathrm{p}$-range: $\left.0.01-9.5 \times 10^{-14}\right)$ and over-represented in the enhancer and DNAse hypersensitive regions ( $\mathrm{p}$ range: $0.05-1.7 \times 10^{-15}$ ]. However, in the $\mathrm{B}_{12}+$ folic acid group, DMCpGs were underrepresented in the gene body, S-shelf and S-shore ( $p$ range: 0.03-0.01) and over-represented in TS200, nongene and promoter-associated regulatory regions ( $\mathrm{p}$ range: $1.6 \times 10^{-3}-1.5 \times 10^{-4}$; Figure $2 \mathrm{E}-\mathrm{H}$; Supplementary 


\begin{tabular}{|c|c|c|c|}
\hline Groups & Control & $B_{12}$ & MMN \\
\hline \multicolumn{4}{|l|}{ Parameters } \\
\hline N (Male/female) & $55(30 \mathrm{M} / 25 \mathrm{~F})$ & $54(23 \mathrm{M} / 31 \mathrm{~F})$ & $55(18 \mathrm{M} / 37 \mathrm{~F})$ \\
\hline Age (years) & $11.3(11.0-12.0)$ & $11.4(10.9-11.8)$ & $11.4(10.9-12.2)$ \\
\hline \multicolumn{4}{|l|}{ Height (cm) } \\
\hline Baseline & $140.6(135.0-145.5)$ & $140.3(134.0-144.6)$ & $138.1(135.0-143.0)$ \\
\hline Supplementation & $145.6(139.0-150.0)$ & $144.9(138.1-149.0)$ & $143.8(140.0-147.8)$ \\
\hline Change & $4.3(3.6-5.2)$ & $4.1(3.3-5.0)$ & $4.5(3.9-5.3)$ \\
\hline \multicolumn{4}{|l|}{ Weight (kg) } \\
\hline Baseline & $28.2(25.0-34.0)$ & $30.0(25.8-33.1)$ & $28.8(25.0-33.4)$ \\
\hline Supplementation & $30.6(27.3-39.1)$ & $32.5(28.2-36.6)$ & $32.7(27.4-37.3)$ \\
\hline Change & $2.7(1.9-4.4)$ & $2.9(2.0-4.2)$ & $3.1(2.0-4.8)$ \\
\hline \multicolumn{4}{|l|}{ BMI $\left(\mathrm{kg} / \mathrm{m}^{2}\right)$} \\
\hline Baseline & $14.6(13.6-16.6)$ & $14.9(14.1-16.5)$ & $15.0(13.8-16.3)$ \\
\hline Supplementation & $15.1(14.0-17.4)$ & $15.3(14.6-17.2)$ & $15.3(14.3-17.2)$ \\
\hline Change & $0.4(0.1-1.1)$ & $0.5(0.2-0.9)$ & $0.7(0.2-1.1)$ \\
\hline \multicolumn{4}{|l|}{$\mathrm{B}_{12}(\mathrm{pmol} / \mathrm{l})$} \\
\hline Baseline & $183.0(141.0-230.0)$ & $187.5(156.0-226.5)$ & $173.0(132.0-224.0)$ \\
\hline Supplementation & $192.0(135.5-276.5)$ & $289.0(212.0-383.5)$ & $251.0(210.0-310.0)$ \\
\hline Change & $5.0(-26.5-44.5)$ & $91.0(47.0-167.0)^{\dagger * * * *}$ & $82.0(33.0-129.0)^{\dagger * * * *}$ \\
\hline \multicolumn{4}{|l|}{ Folate $(\mathrm{nmol} / \mathrm{l})$} \\
\hline Baseline & $20.8(15.6-27.3)$ & $19.6(16.1-22.8)$ & $19.0(15.7-24.8)$ \\
\hline Supplementation & $21.4(17.0-28.2)$ & $20.7(16.0-32.0)$ & $45.8(35.0-58.3)$ \\
\hline Change & $0.8(-2.0-5.1)$ & $1.3(-1.8-8.5)^{*}$ & $27.8(18.4-37.4)^{\dagger * * * *}$ \\
\hline \multicolumn{4}{|c|}{ Homocysteine $(\mu \mathrm{mol} / \mathrm{l})$} \\
\hline Baseline & $16.4(14.3-21.3)$ & $15.7(12.6-20.7)$ & $17.0(13.1-20.6)$ \\
\hline Supplementation & $18.9(15.6-24.1)$ & $14.2(12.5-16.3)$ & $12.7(10.6-14.4)$ \\
\hline Change & $1.9(0.2-4.2)^{* * * *}$ & $-1.4(-5.0-0.1)^{\dagger * * * *}$ & $-3.8(-8.1-1.7)^{\dagger * * * *}$ \\
\hline \multicolumn{4}{|c|}{$\begin{array}{l}\text { All values are median and IQR (interquartile range). Significance of change within the group }\left({ }^{*} \mathrm{p} \leq 0.05 ;{ }^{* *} \mathrm{p} \leq 0.01 ;{ }^{* * *} \mathrm{p} \leq 0.001 ; * * * * \mathrm{p} \leq 0.0001\right) \text {. } \\
\text { † Significant difference when compared with the placebo group. } \\
\mathrm{B}_{12}: \mathrm{B}_{12} 2 \mu \mathrm{g} \text {; Control: No supplementation; MMN: Multiple micronutrient }\left(\mathrm{B}_{12} 1.8 \mu \mathrm{g} \text {, folic acid } 300 \mu \mathrm{g}\right) \text {; } \mathrm{N} \text { : Number. }\end{array}$} \\
\hline
\end{tabular}

\section{Table 3. List of differentially methylated CpGs and regions in different supplementation and analysis groups in the Pune} Maternal Nutrition Study cohort.

\begin{tabular}{|c|c|c|}
\hline \multirow[t]{2}{*}{ Groups } & DMCpGs & DMRs \\
\hline & FDR $<0.02$, Beta_diff $>5 \%$ & $\min \mathrm{FDR}<0.02$, meanbetafc $>2 \%$ \\
\hline Placebo (B0F0) & 12 & 18 \\
\hline Folic acid (B0F200) & 19 & 27 \\
\hline $\mathrm{B}_{12}$ (B10F0) & 589 & 2891 \\
\hline $\mathrm{B}_{12}+$ folic acid (B10F200) & 169 & 3241 \\
\hline Without_B 12 (BOF200 + BOF0) & 519 & 1725 \\
\hline Pooled_B 12 (B10F200 + B10F0) & 8609 & 3911 \\
\hline \multicolumn{3}{|c|}{ 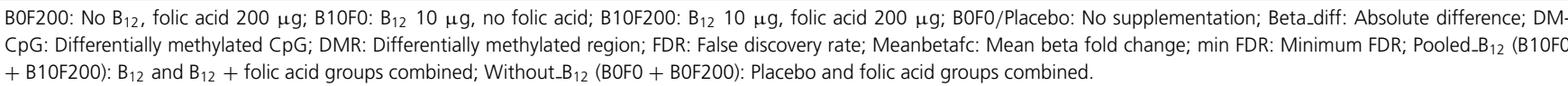 } \\
\hline
\end{tabular}

Table 13). IPA software was used to perform pathways analysis of the genes containing the DMCpGs identified above. We observed significant enrichment of canonical pathways reportedly having a role in T2D, such as estrogen receptor signaling, adipogenesis pathways, glycogen biosynthesis II in the $\mathrm{B}_{12}$ group along with other important pathways related to molecular and cellular function, physiology and development, cardiotoxicity, hepatotoxicity and nephrotoxicity (Supplementary Table 14A). Similarly, pathways significantly enriched in the $\mathrm{B}_{12}+$ folic acid 
group were Cell Cycle - G2/M DNA Damage Checkpoint Regulation, and Wnt/-catenin Signaling. Several other pathways related to molecular and cellular function, physiology and development, cardiotoxicity, hepatotoxicity and nephrotoxicity were also enriched in the $\mathrm{B}_{12}+$ folic acid group (Supplementary Table $14 \mathrm{~B}$ ). This indicates that the identified DMCpGs are enriched in pathways related to regulation of development and glucose and lipid metabolism.
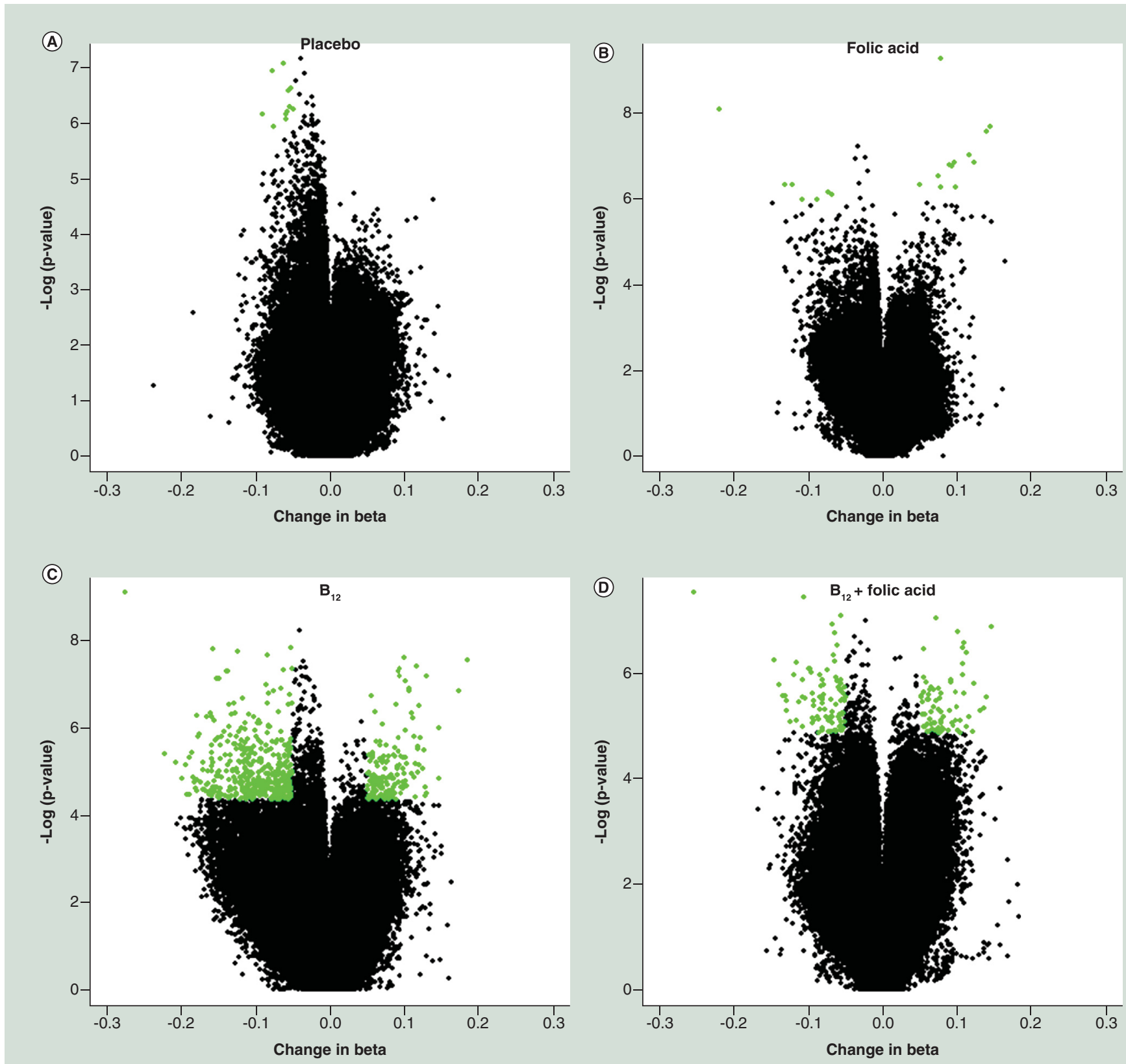

Figure 2. Differentially methylated positions in four supplementation groups and their distribution across different genomic features. Volcano plots show the changes in DNA methylation after supplementation with (A) placebo (B) folic acid (C) $B_{12}$ and (D) $B_{12}+$ folic acid. The black dots represent all the probes investigated and the green dots represent beta differences of $5 \%$ (methylation changes) and adjusted $p$-value $<0.02$. A total of 589 significant probes in $B_{12}$ group and 169 probes in $B_{12}+$ folic acid group were analyzed and plotted for their distribution across (E) genic features, (F) CpG island features, (G) regulatory features and (H) gene and nongenic features, respectively. 


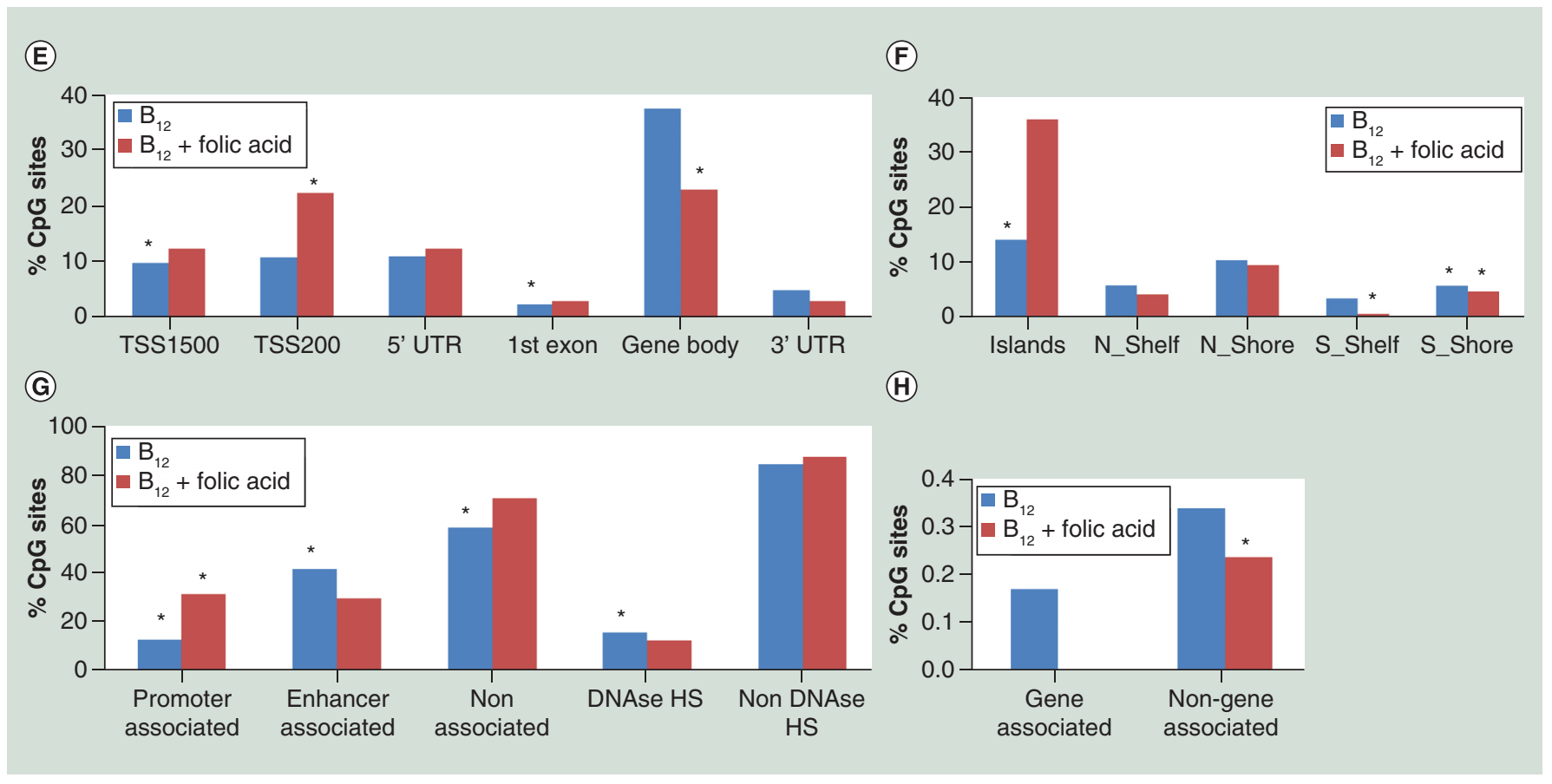

Figure 2. Differentially methylated positions in four supplementation groups and their distribution across different genomic features (cont.).

Volcano plots show the changes in DNA methylation after supplementation with (A) placebo (B) folic acid (C) $B_{12}$ and (D) $B_{12}+$ folic acid. The black dots represent all the probes investigated and the green dots represent beta differences of $5 \%$ (methylation changes) and adjusted $p$-value $<0.02$. A total of 589 significant probes in $B_{12}$ group and 169 probes in $B_{12}+$ folic acid group were analyzed and plotted for their distribution across (E) genic features, (F) CpG island features, (G) regulatory features and (H) gene and nongenic features, respectively.

\begin{tabular}{|c|c|c|c|c|c|c|c|c|c|c|c|c|}
\hline \multirow{2}{*}{$\begin{array}{l}\text { Groups } \\
\text { Gene/probe id }\end{array}$} & \multicolumn{2}{|c|}{ Placebo (BOF0) } & \multicolumn{2}{|c|}{ Folic acid (B0F200) } & \multicolumn{2}{|l|}{$\mathrm{B}_{12}(\mathrm{~B} 10 \mathrm{~F} 0)$} & \multicolumn{2}{|c|}{$\begin{array}{l}\text { B }_{12}+\text { folic acid } \\
\text { (B10F200) }\end{array}$} & \multicolumn{2}{|c|}{$\begin{array}{l}\text { Without_B }{ }_{12} \text { (BOF0 } \\
\text { and BOF200) }\end{array}$} & \multicolumn{2}{|c|}{$\begin{array}{l}\text { Pooled_B } 12 \text { (B10F0 and } \\
\text { B10F200) }\end{array}$} \\
\hline & $\begin{array}{l}\text { FDR } \\
(<0.02)\end{array}$ & $\begin{array}{l}\text { Beta diff } \\
(\%)\end{array}$ & $\begin{array}{l}\text { FDR } \\
(<0.02)\end{array}$ & $\begin{array}{l}\text { Beta diff } \\
(\%)\end{array}$ & $\begin{array}{l}\text { FDR } \\
(<0.02)\end{array}$ & $\begin{array}{l}\text { Beta diff } \\
(\%)\end{array}$ & $\begin{array}{l}\text { FDR } \\
(<0.02)\end{array}$ & $\begin{array}{l}\text { Beta diff } \\
(\%)\end{array}$ & $\begin{array}{l}\text { FDR } \\
(<0.02)\end{array}$ & $\begin{array}{l}\text { Beta diff } \\
(\%)\end{array}$ & $\begin{array}{l}\text { FDR } \\
(<0.02)\end{array}$ & $\begin{array}{l}\text { Beta diff } \\
(\%)\end{array}$ \\
\hline TCF7L2/ cg03683087 & $x$ & $x$ & $x$ & $x$ & 0.016 & 5.50 & $x$ & $\mathrm{x}$ & $x$ & $x$ & $1.0 \times 10^{-3}$ & 4.30 \\
\hline FTO/ cg26580413 & $x$ & $x$ & $x$ & $x$ & $x$ & $x$ & $x$ & $x$ & $x$ & $x$ & $1.2 \times 10^{-3}$ & 6.20 \\
\hline GALNT2/ cg00589617 & $\mathrm{x}$ & $x$ & 0.0125 & 7.80 & $x$ & $x$ & 0.014 & 7.10 & $7.0 \times 10^{-3}$ & 6.50 & $9.1 \times 10^{-7}$ & 7.60 \\
\hline $\begin{array}{l}\text { PPARGC1B/ } \\
\text { cg08928958 }\end{array}$ & $x$ & $x$ & $x$ & $x$ & $x$ & $\mathrm{x}$ & $x$ & $x$ & $x$ & $x$ & $5.0 \times 10^{-3}$ & 7.20 \\
\hline $\operatorname{miR} 21^{\dagger}$ & $\mathrm{x}$ & $x$ & $x$ & $x$ & $1.5 \times 10^{-5}$ & 4.30 & $3 . \times 10^{-8}$ & 4.50 & $1.3 \times 10^{-8}$ & 3.50 & $8.0 \times 10^{-15}$ & 4.20 \\
\hline$S K I^{\dagger}$ & $x$ & $x$ & $x$ & $x$ & $x$ & $x$ & $x$ & $x$ & $x$ & $x$ & $7.0 \times 10^{-4}$ & 4.70 \\
\hline
\end{tabular}

Technical validation \& investigation of selected differentially methylated CpGs \& regions in the Chikki Trial

Based on the above results, we selected loci for replication analysis; if they had attained FDR $<0.02$ in comparative pooled analysis of groups with and without $B_{12}$ supplementation (Table 4), based on their biological relevance and the established genetic association with T2D and associated intermediate phenotypes such as obesity and insulin resistance. Several T2D-associated genes such as TCF7L2, FTO, PPARGC1B, GALNT2, IGF2BP2, KCNQ1, etc. passed the FDR $<0.02$ (Supplementary Table 15). Four DMCpGs, located within FTO (cg26580413), TCF7L2 
(A)

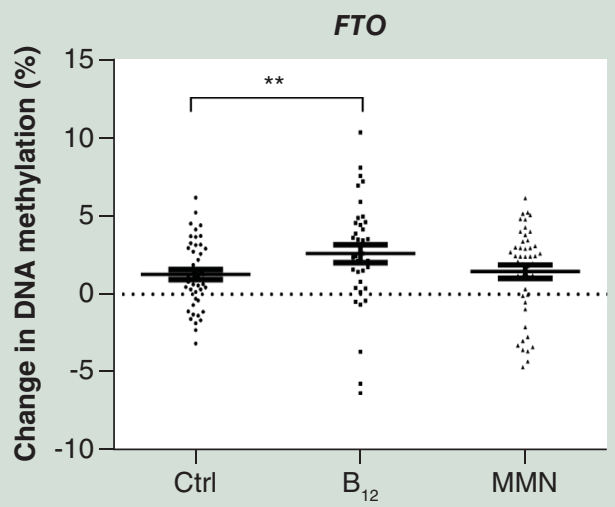

(C)

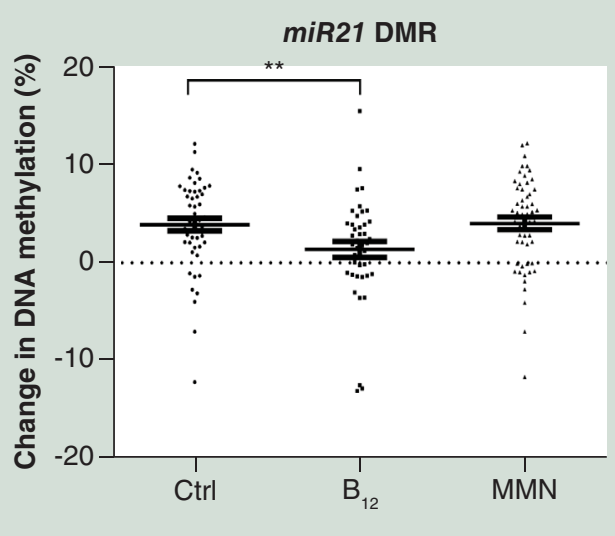

(B)

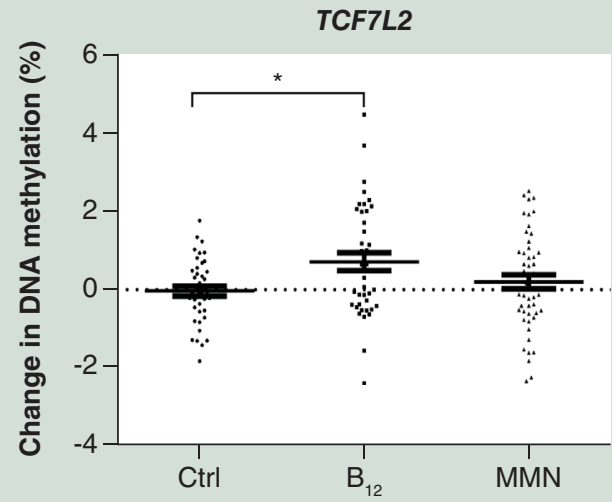

(D)

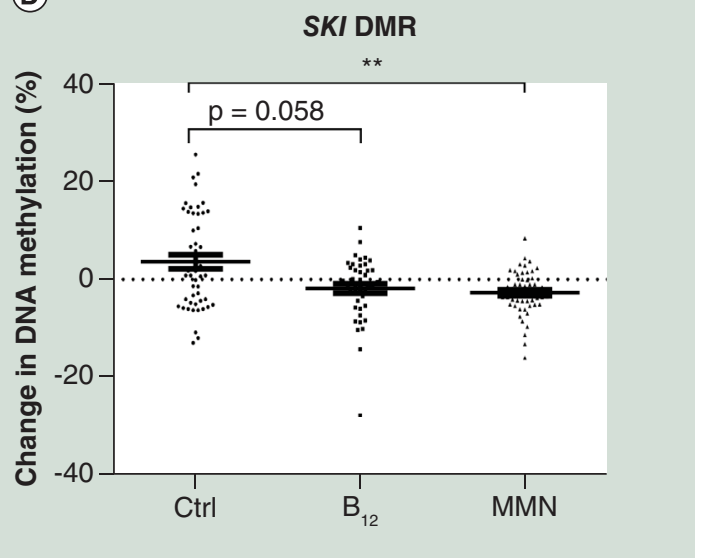

Figure 3. Investigation of selected differentially methylated $\mathrm{CpGs}$ and regions by pyrosequencing in the Chikki Trial. Two DMCpG loci (FTO and TCF7L2) and two DMRs (miR21 and SKI) were selected for replication in the Chikki Trial. Changes in DNA methylation in different groups are shown for (A) FTO, (B) TCF7L2, (C) miR21 and (D) SKI. ${ }^{*} \mathrm{p} \leq 0.05 ;{ }^{*} \mathrm{p} \leq 0.01$, all data presented as mean \pm standard error of the mean.

Ctrl: Control; DMCpG: Differentially methylated CpG; DMR: Differentially methylated region; MMN: Multiple micronutrient.

(cg03683087), PPARGC1B (cg08928958) and GALNT2 (cg00589617) genes and two DMRs (consisting of multiple consecutive positions) located within miRNA 21 ( $m i R 21)$ and SKI. Mean methylation difference at these loci within groups with $\mathrm{B}_{12}$ supplementation ranged from 4.20 to $7.60 \%$ (Table 4). First, we performed technical validation of the Infinium HumanMethylation450 BeadChip Array results for four DMCpGs mentioned above. While the finding of significant differential methylation within $F T O$ and TCF7L2 loci was technically validated, no significant methylation differences were observed at the PPARGC1B and GALNT2 loci (Supplementary Figure 1). Association analysis of methylation levels at CpGs in FTO and TCF7L2 with SNPs in a $50 \mathrm{~kb}$ region on either side did not show any significant association suggesting no effect of genotype at these CpGs (Supplementary Table 16). Thus, two DMCpGs (FTO and TCF7L2; both hypomethylated), and two DMRs, miR21 (hypermethylated) and $S K I$ (hypomethylated) were investigated in the replication analysis in the Chikki Trial subjects.

In the Chikki Trial samples, the mean methylation levels increased by $1.92 \%(\mathrm{p}=0.004)$ at $F T O(\mathrm{cg} 26580413)$ and by $0.58 \%(\mathrm{p}=0.012)$ at TCF7L2 $(\mathrm{cg} 03683087)$ after supplementation in the $\mathrm{B}_{12}$ group (Figure $3 \mathrm{~A} \& \mathrm{~B}$ ) but no statistically significant methylation differences were noted in the MMN group ( $\mathrm{p}>0.05$ ), compared with the placebo group. Further, a stratified analysis by sex revealed a median $1.5 \%$ increase in methylation at $T C F 7 L 2$ in males compared with females in the $\mathrm{B}_{12}$ group $(\mathrm{p}=0.0014)$ but no significant gender-specific differences were observed in the FTO locus (Supplementary Figure 2). Of the 12 CpGs in the miR21 DMR, we analyzed the region spanning CpGs $1-5$ near the transcription start site and identified $2.60 \%$ lower mean methylation levels $(p=0.004)$ in the $B_{12}$ group compared with the placebo group (Figure 3C). Similarly, the mean percentage of 
methylation of two CpG analyzed in the SKI DMR was $1.96 \%$ lower $(\mathrm{p}=0.058)$ in the $\mathrm{B}_{12}$ group than in the placebo group (Figure 3D). Comparing the placebo and the MMN group, we found no significant methylation changes at miR21 DMR ( $>0.05)$ but the SKI DMR showed a significant methylation difference of $3.31 \%$ ( $\mathrm{p}<$ 0.006). Overall, we observed significant hypermethylation in the FTO and TCF7L2 and hypomethylation in the miR21 and SKI DMRs after $\mathrm{B}_{12}$ supplementation in the Chikki Trial. While the mean methylation differences were similar at these loci in Chikki trial, the directionality was not same as in the PMNS cohort except for SKI locus. FTO and TCF7L2 are established candidate genes for obesity and T2D [25,26]. Similarly, miR21 has a regulatory role in OCM and complex metabolic diseases [27-29] and SKI is known to influence diet-induced obesity, body composition and lipid metabolism [30,31]. These observations suggest that $\mathrm{B}_{12}$ supplementation influences methylation at the above loci associated with T2D and related intermediate traits.

The miR21 differentially methylated region exhibits promoter activity that is suppressed by methylation of CpG1-4 region

In order to investigate the functional importance of differential methylation of CpGs in miR21 DMR, we generated two reporter gene constructs by cloning the miR21 promoter region (-344 bp to +590 bp; miR21_Pro_pGL3B) and a 246 bp region covering only the CpG1-4 in miR21 DMR $(+344$ bp to +590 bp with respect to the transcription start site; miR21_DMR_pGL3B) into a luciferase expression plasmid and performed reporter assay after transfecting them into three different cell lines (Figure 4A). Both constructs showed significant promoter-like activity. Compared with the miR21 promoter, the miR21 DMR exhibited 45, 47 and 63\% promoter activity in HEK293, HepG2 and MIN6 cell lines, respectively (Figure 4B-D). Hence, further experiments on the effect of methylation on promoter activity of miR21 DMR were conducted by transfecting the methylated and mock methylated miR21_DMR_pGL3B construct into the HEK293 cell line. The methylated miR21 DMR showed significantly less promoter activity than the mock methylated construct in the HEK293 cell line (25\% less; p < 0.001) (Figure 4E). Furthermore, on electrophoretic mobility shift assay using a methylated and mock methylated DMR sequence and HEK293 nuclear extract, we observed stronger interaction with the methylated DMR, indicating that DNA methylation of miR21DMR affects interactions with putative transcription factor(s) (Figure 4F). In competition assays, nonspecific probes did not affect binding to the DMR (data not shown) suggesting that the interactions between the DMR and putative transcription factor were specific and modulated by methylation.

Genes associated with Type 2 diabetes \& related traits are direct targets of miR21-3p

We used three different miRNA target prediction databases and tools, viz. miRDB, miRanda and DIANA to predict target genes of miR21. Of the several hits, four potential targets of miR21-3pFTO, TCF7L2, cAMP response element binding protein (CREB) binding protein, (CREBBP/CBP) and Sirtuin1 (SIRT1) were selected for further study. The targets were selected based on prediction by more than one tool, conserved binding sequences for $m i R 21-3 p$ and biological significance, especially with relevance to T2D and related intermediate traits. Co-transfection of FTO, TCF7L2, CREBBP and SIRT1 target constructs and miR21-3p overexpressing constructs in HepG2 cell line followed by reporter assays showed that overexpression of $m i R 21-3 p$ reduced the reporter activity of $F T O$ ( $48 \%$; $\mathrm{p}<0.0002)$, TCF7L2 (30\%; $\mathrm{p}<0.03)$, CREBBP (40\%; $\mathrm{p}<0.01)$ and SIRT1 (44\%; $<<0.0006)$, respectively (Figure 5A-D). To further confirm that these genes are direct targets of miR21-3p, we mutated the seed sequence of the target construct by site-directed mutagenesis (Figure $5 \mathrm{E}$ ) and observed that the seed sequence mutation abolished the effect of miR21-3p overexpression-dependent reduction of target reporter assay (Figure 5F-I). These observations confirm that miR21-3p directly regulates the expression of all four genes, TCF7L2, FTO, CREBBP and SIRT1.

\section{Discussion}

The study was driven by two important considerations: vitamin $\mathrm{B}_{12}$ deficiency is very common in Indians [7], and it may be associated with an increased risk of diabetes and cardiovascular disease in an intergenerational manner (fetal programming) [10,11]. We investigated the molecular changes associated with $\mathrm{B}_{12}$ supplementation, alone and with folic acid, in adolescent subjects from a $B_{12}$-deficient but folate-sufficient population and made several important observations. First, $B_{12}$ supplementation (alone and with folic acid) but not folic acid supplementation alone, led to DNA methylation changes throughout the genome. Second, supplementation influenced the methylation levels in several metabolically important genes or their regulators. An exciting finding was that one of the DMRs identified within miR21 regulates the expression of many genes implicated in T2D such as TCF7L2 and FTO which were 
(A) miR21_Pro-pGL3B construct

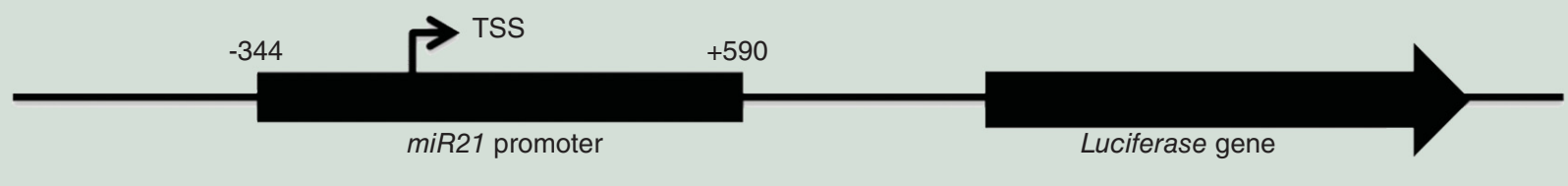

miR21_DMR-pGL3B construct

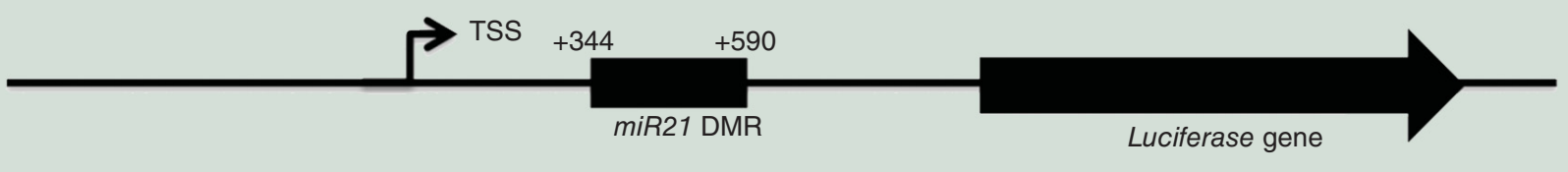

(B)

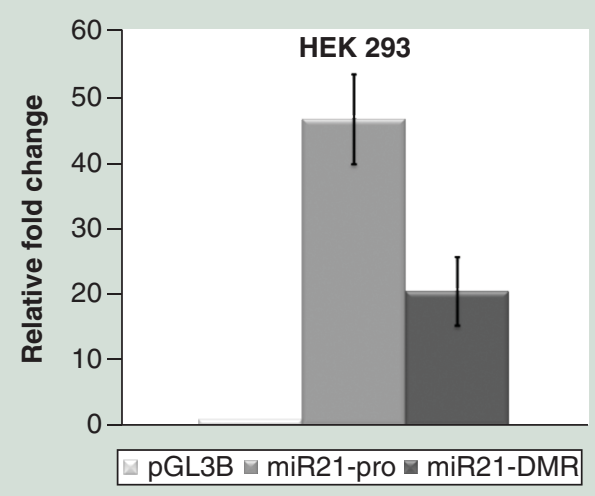

(E)
(C)

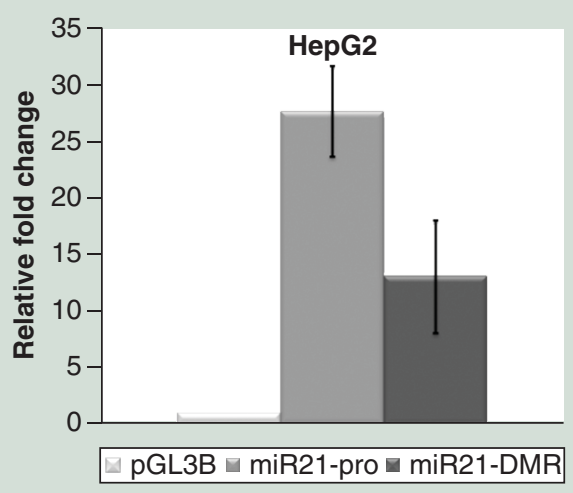

(F)
(D)

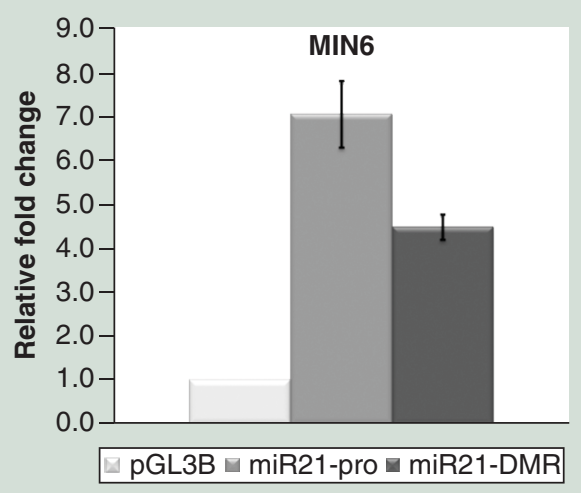

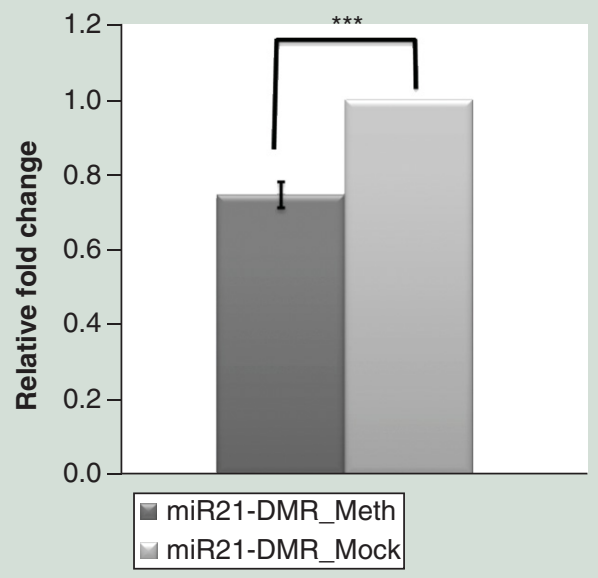

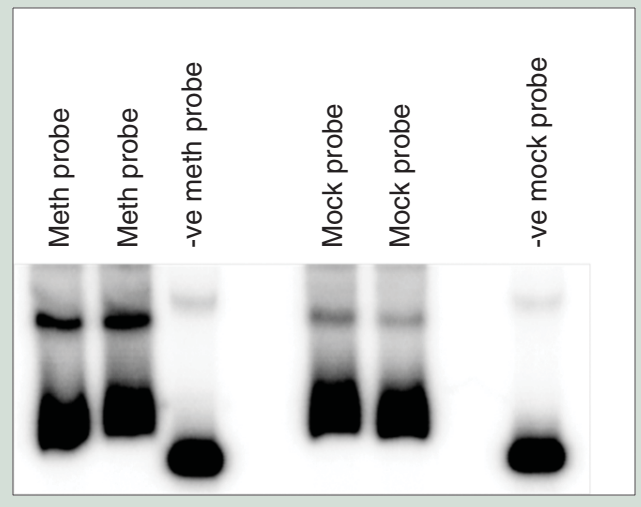

Figure 4. Functional characterization of miR21 differentially methylated region. Schematic representation of miR21 promoter and miR21 DMR clones, in basic luciferase reporter vector (pGL3B) (A). Relative promoter activity of miR21-DMR in (B) HEK 293, (C) HepG2 and (D) MIN6 cell lines. (E) Effect of methylation on promoter activity of miR21-DMR. (F) Differential interaction of putative transcription factor(s) with methylated and mock methylated miR21-DMR assessed by electrophoretic mobility shift assay.

$* * * \mathrm{p} \leq 0.001$, all data presented as mean \pm standard error of the mean.

DMR: Differentially methylated region.

hypermethylated upon $\mathrm{B}_{12}$ supplementation in both the PMNS and Chikki cohorts. Thus, we have identified a novel epigenetic mechanism mediated by miR21 that may be a link between $\mathrm{B}_{12}$ nutrition and the associated OCM with risk of $\mathrm{T} 2 \mathrm{D}$ and adiposity. 


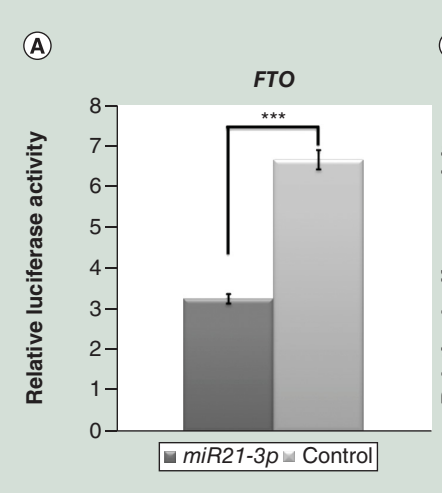

(B)

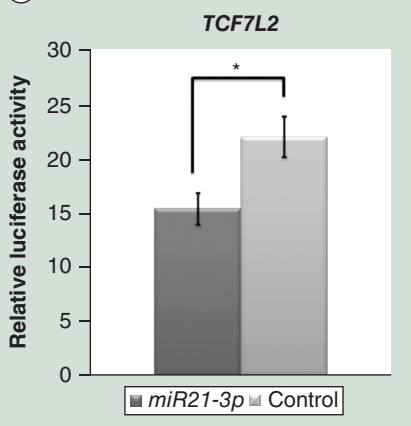

(C)

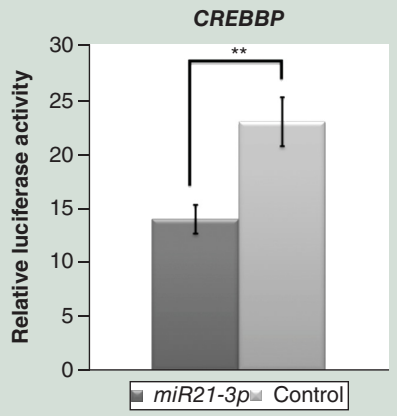

(D)

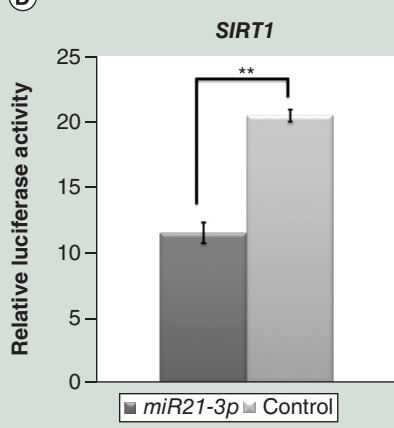

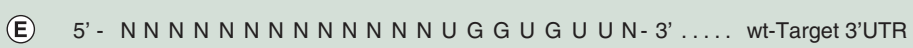

IIIIII|

3'- U GU C G GGUAG C U G A C C A C A A C - 5, .... hsa-miR-21-3p

II I

$5^{\prime}-$ N N N N N N N N N N N N N C G G A A U C N - 3'.... mut-Target 3'UTR

(F)

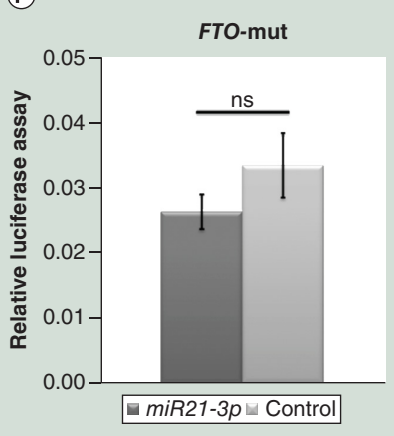

(G)

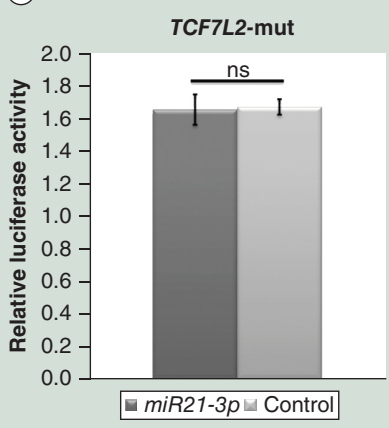

(H)

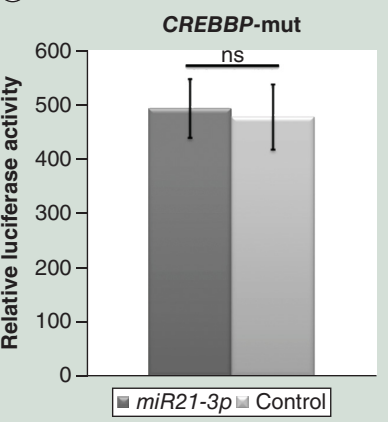

(1)

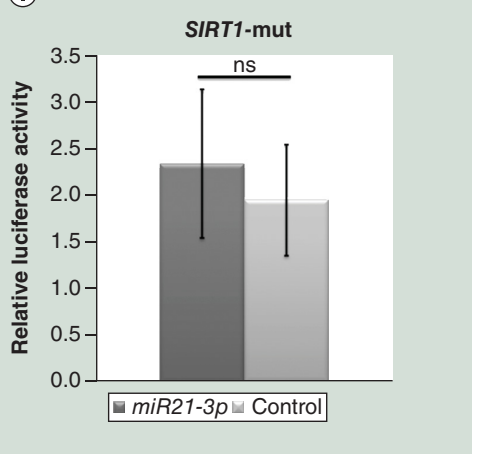

Figure 5. Validation of predicted miR21-3p targets by luciferase reporter assay. Overexpression of miR21-3p (miR21-3p-pmU6) significantly reduces relative expression of reporter gene containing putative binding sequence of predicted targets in comparison to control (control-pmU6) (A) FTO, (B) TCF7L2, (C) CREBBP and (D) SIRT1. (E) Schematic representation of putative binding site for miR21-3p in $3^{\prime}$-UTR of the target genes (top), mutation of the seed sequences of target genes (bottom). Mutation of seed sequences abolishes the effect of miR21-3p overexpression on (F) FTO-mut, (G) TCF7L2-mut, (H) CREBBP-mut and (I) SIRT1-mut in HepG2 cell line. mut, indicates the respective mutated constructs. All data presented as mean \pm standard error of the mean.

${ }^{*} \mathrm{p} \leq 0.05 ; * * \mathrm{p} \leq 0.01, * * * \mathrm{p} \leq 0.001$.

ns: Nonsignificant.

Methylation changes occur with $\mathrm{B}_{12}$ supplementation \& not with folic acid alone

Vitamins $B_{12}$ and folic acid regulate the one-carbon metabolic pathway by acting on the same enzyme (methionine synthase). Both play a role in determining SAM and S-adenosyl homocysteine (SAH) levels, and their deficiency is known to influence the SAM/SAH ratio [32,33], which is often used as an indicator for cellular methylation potential [34-36]. Several studies have reported individual effects of folic acid and $\mathrm{B}_{12}$ supplementation on genomewide DNA methylation [37,38]. Hence, it was interesting to note that $\mathrm{B}_{12}$ supplementation significantly influenced DNA methylation, both alone and when given with folic acid. Folic acid alone only induced changes in a small number of DMCpGs, a similar number to that observed in the placebo group suggesting that these may reflect change over time or noise. An earlier study in young Australian adults also reported that folate-sufficient and marginally $\mathrm{B}_{12}$-deficient individuals did not show any significant methylation changes on supplementation with folic acid [39]. Many other recent studies have also failed to demonstrate any effect of folic acid supplementation on DNA methylation in moderately hyperhomocysteinemic subjects [40,41]. As mentioned earlier, the Indian 
population has high homocysteine levels predominantly due to low $\mathrm{B}_{12}$ status, which may explain the lack of genome-wide significant methylation changes with only folic acid supplementation in our study.

\section{$B_{12}$ supplementation influences methylation of Type 2 diabetes candidate genes}

The methylation changes in the $B_{12}$ supplementation groups ( $B_{12}$ alone and $B_{12}+$ folic acid) were widespread across the genome, including the gene body, intergenic regions, $\mathrm{CpG}$ islands, enhancer and promoter-associated features, which corroborates earlier observations that methylation changes are not restricted to any specific region of the genome [42]. Interestingly, the genes that showed differential DNA methylation patterns were related to glucose and lipid metabolism, as exemplified by differential methylation of TCF7L2 and FTO, the strongest candidate genes for T2D and obesity, respectively and many others [25,26]. This observation is in line with earlier findings showing an association between $\mathrm{B}_{12}$ deficiency and lipid metabolism [43], and glucose [44] and cholesterol biosynthesis [45]. An earlier study by Dayeh et al. has reported differential methylation of several T2D loci including FTO and TCF7L2 in pancreatic islets of diabetic and nondiabetic individuals [46]. We observed differential methylation of the same CpG site cg26982104 in FTO, which was reported to be differentially methylated in pancreatic islets. Similar methylation differences were also noted in our study for several T2D loci such as ADCY5, PPAR $\gamma$, IGF2BP2, $K C N Q 1$, etc. that were reported by Dayeh et al. Another study based on T2D candidate genes has reported increased DNA methylation at FTO obesity susceptibility haplotype in females susceptible to T2D [47]. Thus, these findings provide a potential link between $\mathrm{B}_{12}$-mediated differential methylation of $F T O$ and its association with T2D. It was interesting to note gender-specific differences in TCF7L2 methylation, which need to be studied further in view of recent studies that have shown sex-specific differences in both DNA methylation and expression contributing to altered insulin secretion in human islets [48].

$B_{12}$ supplementation regulates metabolically important genes by influencing methylation of miR21 In addition to the differential methylation of $F T O$ and $T C F 7 L 2$, this study for the first time identified hypermethylation of a genomic stretch near the promoter region of miR21, as a result of $\mathrm{B}_{12}$ supplementation both alone and with folic acid. While the role of miRNAs in cancer has been extensively studied, recent studies propose an important role of miRNAs in $\beta$-cell function, T2D and cardiovascular disorders [49]. Several studies using diet and methyl donor supplementation in animals have reported modulation of miRNA expression in liver and adipose tissue [29,50]. A recent study reported hypomethylation of the same set of CpGs (cg16936953, cg12054453, cg01409343 and cg02782634) at the miR21 locus in Crohn's disease, and increased expression of miR21 in the peripheral blood [51]. This is in agreement with our 'in vitro' results, which demonstrate reduced expression of miR21 due to hypermethylation and increased interaction between the methylated miR21-DMR and a putative transcription factor. Although earlier studies had suggested that DNA methylation blocked transcription factor binding, a recent study has shown that DNA methylation can promote transcription factor binding and does so for about a third of transcription factors [15]. Since miR21 methylation suppressed promoter activity, the putative transcription factor could be a transcriptional repressor, whose identification needs further investigation. We validated four target genes of miR21-3p, TCF7L2, FTO, CREBBP and SIRT1, which have established importance in obesity, insulin function, glucose and lipid metabolism. While genetic variants in TCF7L2 are strongly associated with T2D and it acts as the master regulator of $\beta$-cell function, insulin production, processing and secretion [52], the significance of FTO in appetite, dietary habits and obesity induction is well-known [53]. Similarly, the CREB binding protein regulates insulin-mediated hepatic gluconeogenesis, insulin resistance in obesity and insulin sensitivity [54-56] and SIRT1 is known to influence glucose/ lipid metabolism, insulin secretion, signaling, inflammation and oxidative stress [57]. It is worth noting that all four target genes showed significant methylation differences upon supplementation at FDR $<0.02$ in the pooled group analysis; the effect size was smaller for CREBBP and SIRT1. This suggests a novel regulatory role for miR21 methylation in T2D pathways and associated intermediate traits like obesity, insulin resistance and secretion.

Link between $\mathrm{B}_{12}$-mediated miR21 methylation \& regulation of metabolically important genes Recent human and animal studies have reported the potential importance of maternal OCM and nutrients regulating it $\left(\mathrm{B}_{12}\right.$, folate, etc.) in the programming of metabolic diseases in their offspring $[11,43]$. Our observation of differential methylation of a set of T2D candidate genes, especially TCF7L2 and FTO and miR21 by $\mathrm{B}_{12}$ supplementation in a $\mathrm{B}_{12}$-deficient population suggests a possible molecular mechanism for these observations. We can only speculate about the possible mechanism/pathway that could link the methylation changes observed in the 
above loci. The SAM/SAH ratio determines the methylation potential of the cell, and conversion of methionine to SAM is regulated by two key enzymes, methionine adenosyltransferase 2A and 2B (MAT2A and MAT2B), which are established targets of miR21-3p [28]. Thus, differential methylation and expression of metabolically important genes like TCF7L2, FTO, CREBPB and SIRT1 could occur as an effect of miR21 on MAT2A and MAT2B, which regulate the SAM levels. This indicates that miR21 may be the key regulator functioning in a two-pronged way, orchestrating the methylation potential of the cell as well as regulating expression of key genes of metabolic importance.

\section{Strengths \& limitations of the study}

This is the first study to investigate the effects of $\mathrm{B}_{12}$ and folic acid supplementation on the methylome of adolescents. Our study population is multigenerationally undernourished and suffers from MMN deficiencies. The $\mathrm{B}_{12}$-deficient and folate-sufficient status is similar to many other vegetarian populations, making our findings important for other similarly affected populations. However, there are a few limitations in the study. Our observations are based on methylation data on genomic DNA from peripheral blood cells, and therefore may not be directly extrapolated to other tissues. However, the commonality of many sites of differential methylation in blood cells and metabolic tissues (e.g., pancreatic islet cells) lends support to their importance. We have corrected for PCs derived from the cell composition to account for differences in methylation due to cellular heterogeneity, collinearity of blood cell counts and consequent inflation in our study. The methylation changes in our study are relatively small but like genetic studies, the contribution of individual methylation differences to complex disease phenotypes is likely to be small [58]. In view of a small discovery cohort, we used a stringent FDR cut-off $\mathrm{p}<0.02$ and analyzed loci which passed the criteria in the combined $\mathrm{B}_{12}$ group analysis $\left(\mathrm{B}_{12}\right.$ and $\mathrm{B}_{12}+$ folic acid group). One of the differences in the findings in the discovery and validation studies is the contrasting directionality of the DMCpGs and DMRs. Though it is difficult to explain the mechanism, there are several studies which report contrasting methylation changes in similar but different supplementation studies both in animals and humans [59]. We speculate that these contrasting methylation changes may be due to differences in the structure, design, age, dose and duration of $\mathrm{B}_{12}$ and folic acid supplementation in two cohorts. We have made similar observations on methylation changes with different dose of folic acid in an independent animal study (data not shown). Since FTO and TCF7L2 are strongly associated with T2D and obesity, there is a possibility that the methylation changes may be the effect of SNPs near the CpGs at the two loci. Comparison of the genotype data from a $1.2 \mathrm{~kb}$ flanking region around specific CpGs did not demonstrate any association with variants in this region and $\mathrm{CpG}$ methylation. Therefore, this study along with functional results demonstrates that methylation at these loci is sensitive to $\mathrm{B}_{12}$ supplementation, not likely to be chance findings and generates a hypothesis to be tested in future studies.

\section{Conclusion}

To conclude, we demonstrate that $\mathrm{B}_{12}$ supplementation with and without folic acid in our $\mathrm{B}_{12}$-deficient and folate-sufficient population influences the methylation of key genes implicated in the risk of T2D and related phenotypes. This may be achieved through regulation of OCM via MAT2A and MAT2B by miR21 which offers a novel epigenetic explanation for the association between OCM and risk of noncommunicable diseases. Since $B_{12}$ deficiency is common in elderly population and other vegetarian population, therefore, our findings could have potential public health significance if confirmed in other populations and nutritional trials.

Supplementary data

To view the supplementary data that accompany this paper please visit the journal website at: www.futuremedicine.com/doi/suppl/ 10.2217/epi-2017-0102.

\section{Author contributions}

CS Yajnik and GR Chandak conceptualized and planned the study with significant intellectual contribution from CHD Fall and KA Lillycrop. DK Yadav and S Shrestha performed all high-throughput and functional experiments and wrote the first draft of the manuscript. H Pan, JD Holbrook and S Shrestha performed the analysis of Infinium HumanMethylation450 BeadChip Array data. CV Joglekar performed the statistical analysis of phenotype data from the cohorts. All authors read and provided critical comments on the manuscript. GR Chandak is the guarantor of this work and, as such, had full access to all the data in the study and takes responsibility for the integrity of the data and the accuracy of the data analysis. 
Summary points

- Vitamins $B_{12}$ and folate are critical determinants of one-carbon metabolism necessary for DNA methylation.

- Homocysteine, a summative marker of one-carbon metabolism, is an important risk marker for cardiovascular disease, Type 2 diabetes and metabolic syndrome.

- We have earlier demonstrated significant reduction in plasma homocysteine levels by $B_{12}$ but not folic acid supplementation in the Pune Maternal Nutrition Study.

- In the present study, we have investigated DNA methylation changes in blood samples of children supplemented with different combinations of $\mathrm{B}_{12}$ and folic acid for 1 year.

- Methylation changes were observed only in the groups which received $B_{12}$ (589 differentially methylated CpGs [DMCpGs] and 2892 regions [DMRs]) and $B_{12}$ with folic acid ( $B_{12}+$ folic acid; 169 DMCpGs and 3241 DMRs).

- We observed significant DMCpGs in Type 2 diabetes-associated genes like TCF7L2 and FTO.

- We also identified a DMR within miR21 gene upon supplementation.

- Using in vitro techniques, we demonstrated that methylation of miR21 DMR represses its expression by modulating interactions with putative transcription factors.

- At last, we demonstrated that metabolically important genes like FTO, TCF7L2, CREBBP/CBP and SIRT1 are direct targets of miR21-3p.

Acknowledgements

The authors thank all the participants of all the cohorts for agreeing to join the study and field staff for their contributions in sample collection and community work. The help of S Bhaskar, KR Mani and ID Mali, CSIR-Centre for Cellular and Molecular Biology, Hyderabad in genomic DNA isolation from blood samples and in managing the DNA samples is sincerely acknowledged. The authors acknowledge major contributions by US Deshmukh, S Rao, S Hirve, P Gupta, DS Bhat, H Lubree, S Rege, P Yajnik and the invaluable community work contributed by T Deokar, S Chaugule, A Bhalerao and V Solat from the KEM Hospital Research Centre, Pune.

Availability of data \& material

The summary association statistics from the genome-wide methylation data presented in this study will be made available at the institutional website (www.ccmb.res.in). The results of DMCpGs and DMRs identified in this study using Infinium HumanMethylation450 BeadChip are provided in Supplementary Tables 4-11.

Financial \& competing interests disclosure

This work was supported by funds from Council of Scientific and Industrial Research (CSIR), Ministry of Science and Technology, Government of India, India (XII 5-Year Plan titled "EpiHED; BSC0118"). The PMNS cohort and Chikki Trial were initially supported by funds from the Wellcome Trust, London, UK, Medical Research Council, London, UK and Department of Biotechnology (DBT), Ministry of Science and Technology, Government of India, India, respectively. DK Yadav also acknowledges the support of European Union for support under FP7 funded project GEoCoDe for his exchange visit to University of Southampton, Southampton, UK. The funders had no role in study design, data collection and analysis, decision to publish, or preparation of the manuscript. The authors have no other relevant affiliations or financial involvement with any organization or entity with a financial interest in or financial conflict with the subject matter or materials discussed in the man uscript apart from those disclosed.

No writing assistance was utilized in the production of this manuscript.

Ethical conduct of research

The study was approved by the KEM Hospital Ethics Committee and informed written consent of the parents and informed written assent of the participants has been taken (ref: KEMHRC/VSP/Dir Off/EC/065; Project No. 067).

\section{References}

Papers of special note have been highlighted as: $\bullet$ of interest; $\bullet \bullet$ of considerable interest

1. Smith EL, Parker LF. Purification of antipernicious anaemia factor. Biochem. J. 43(1), viii (1948).

2. Peker E, Demir N, Tuncer $\mathrm{O}$ et al. The levels of vitamin B, folate and homocysteine in mothers and their babies with neural tube defects. J. Matern. Fetal Neonatal Med. 23, 1-5 (2015).

3. Mahalle N, Kulkarni MV, Garg MK, Naik SS. Vitamin B12 deficiency and hyperhomocysteinemia as correlates of cardiovascular risk factors in Indian subjects with coronary artery disease. J. Cardiol. 61(4), 289-294 (2013). 
Research Article Yadav, Shrestha et al.

4. Martos R, Valle M, Morales R, Canete R, Gavilan MI, Sanchez-Margalet V. Hyperhomocysteinemia correlates with insulin resistance and low-grade systemic inflammation in obese prepubertal children. Metabolism 55(1), $72-77$ (2006).

5. Buysschaert M, Dramais AS, Wallemacq PE, Hermans MP. Hyperhomocysteinemia in Type 2 diabetes: relationship to macroangiopathy, nephropathy and insulin resistance. Diabetes Care 23(12), 1816-1822 (2000).

6. Ntaios G, Savopoulos C, Chatzopoulos S, Mikhailidis D, Hatzitolios A. Iatrogenic hyperhomocysteinemia in patients with metabolic syndrome: a systematic review and meta-analysis. Atheroscl. 214(1), 11-19 (2011).

7. Yajnik CS, Deshpande SS, Lubree HG et al. Vitamin B12 deficiency and hyperhomocysteinemia in rural and urban Indians. J. Assoc. Physicians India 54, 775-782 (2006).

- Vitamin $B_{12}$ deficiency is common in Indians and a major determinant of plasma homocysteine levels.

8. Yajnik CS, Chandak GR, Joglekar C et al. Maternal homocysteine in pregnancy and offspring birthweight: epidemiological associations and Mendelian randomization analysis. Int. J. Epidemiol. 43(5), 1487-1497 (2014).

- Mendelian randomization analysis using MTHFR established a causal role for maternal homocysteine concentrations in influencing fetal growth.

9. Bhate V, Deshpande S, Bhat D et al. Vitamin B12 status of pregnant Indian women and cognitive function in their 9-year-old children. Food Nutr. Bull 29(4), 249-254 (2008).

10. Deshmukh U, Katre P, Yajnik CS. Influence of maternal vitamin B12 and folate on growth and insulin resistance in the offspring. Nestle Nutr. Inst. Workshop Ser. 74, 145-154; discussion 154-146 (2013).

11. Yajnik CS, Deshpande SS, Jackson AA et al. Vitamin B12 and folate concentrations during pregnancy and insulin resistance in the offspring: the Pune Maternal Nutrition Study. Diabetologia 51(1), 29-38 (2008).

- Hyperhomocysteinemia due to $B_{12}$ deficiency influences fetal growth, the risk of Type 2 diabetes and cardiovascular disease.

12. Deshmukh US, Joglekar CV, Lubree HG et al. Effect of physiological doses of oral vitamin B12 on plasma homocysteine: a randomized, placebo-controlled, double-blind trial in India. Eur. J. Clin. Nutr. 64(5), 495-502 (2010).

-• $\quad B_{12}$ supplementation, but not folic acid, significantly reduces plasma homocysteine levels in Indians.

13. Rao S, Yajnik CS, Kanade A et al. Intake of micronutrient-rich foods in rural Indian mothers is associated with the size of their babies at birth: Pune Maternal Nutrition Study. J. Nutr. 131(4), 1217-1224 (2001).

14. Aryee MJ, Jaffe AE, Corrada-Bravo $\mathrm{H}$ et al. Minfi: a flexible and comprehensive bioconductor package for the analysis of Infinium DNA methylation microarrays. Bioinformat. 30(10), 1363-1369 (2014).

15. Yin Y, Morgunova E, Jolma A et al. Impact of cytosine methylation on DNA binding specificities of human transcription factors. Science 356(6337), pii:eaaj2239 (2017).

16. Chen YA, Lemire M, Choufani S et al. Discovery of cross-reactive probes and polymorphic CpGs in the Illumina Infinium HumanMethylation 450 microarray. Epigenetics 8(2), 203-209 (2013).

17. Ritchie ME, Phipson B, Wu D et al. limma powers differential expression analyses for RNA-sequencing and microarray studies. Nucleic Acids Res. 43(7), e47 (2015).

18. Jaffe AE, Irizarry RA. Accounting for cellular heterogeneity is critical in epigenome-wide association studies. Genome Biol. 15(2), 2014-2015 (2014).

19. Michael Olusegun Akinwande HGD, Agboola Samson. Variance inflation factor: as a condition for the inclusion of suppressor variable(s) in regression analysis. Open J. Stat. 5, 754-767 (2015).

20. Wong N, Wang X. miRDB: an online resource for microRNA target prediction and functional annotations. Nucleic Acids Res. 43, 5 (2015).

21. Miranda KC, Huynh T, Tay Y et al. A pattern-based method for the identification of microRNA binding sites and their corresponding heteroduplexes. Cell 126(6), 1203-1217 (2006).

22. Kiriakidou M, Nelson PT, Kouranov A et al. A combined computational-experimental approach predicts human microRNA targets. Genes Dev. 18(10), 1165-1178 (2004).

23. Yu JY, Deruiter SL, Turner DL. RNA interference by expression of short-interfering RNAs and hairpin RNAs in mammalian cells. Proc. Natl Acad. Sci. USA 99(9), 6047-6052 (2002).

24. Jones MJ, Islam SA, Edgar RD, Kobor MS. Adjusting for cell-type composition in DNA methylation data using a regression-based approach. Methods Mol. Biol. 1589, 99-106 (2015).

25. Frayling TM, Timpson NJ, Weedon MN et al. A common variant in the FTO gene is associated with body mass index and predisposes to childhood and adult obesity. Science 316(5826), 889-894 (2007).

26. Grant SF, Thorleifsson G, Reynisdottir I et al. Variant of transcription factor 7-like 2 (TCF7L2) gene confers risk of Type 2 diabetes. Nat. Genet 38(3), 320-323 (2006).

27. Arner P, Kulyte A. MicroRNA regulatory networks in human adipose tissue and obesity. Nat. Rev. Endocrinol. 11(5), 276-288 (2015).

28. Lo TF, Tsai WC, Chen ST. MicroRNA-21-3p, a berberine-induced miRNA, directly downregulates human methionine adenosyltransferases 2A and 2B and inhibits hepatoma cell growth. PLoS ONE 8(9), e75628 (2013). 
- Methionine adenosyltransferase 2A and 2B (MAT2A and MAT2B) are established targets of miR21-3p.

29. Kim YJ, Hwang SH, Cho HH, Shin KK, Bae YC, Jung JS. MicroRNA 21 regulates the proliferation of human adipose tissue-derived mesenchymal stem cells and high-fat diet-induced obesity alters microRNA 21 expression in white adipose tissues. J. Cell Physiol. 227(1), 183-193 (2012).

30. Leong GM, Kee AJ, Millard SM et al. The Ski proto-oncogene regulates body composition and suppresses lipogenesis. Int. J. Obes. (Lond.) 34(3), 524-536 (2010).

31. Diaz M, Martel N, Fitzsimmons RL et al. Ski overexpression in skeletal muscle modulates genetic programs that control susceptibility to diet-induced obesity and insulin signaling. Obesity 20(11), 2157-2167 (2012).

32. Bottiglieri T, Laundy M, Crellin R, Toone BK, Carney MW, Reynolds EH. Homocysteine, folate, methylation and monoamine metabolism in depression. J. Neurol. Neurosurg. Psychiatry 69(2), 228-232 (2000).

33. Tolmunen T, Hintikka J, Voutilainen $\mathrm{S}$ et al. Association between depressive symptoms and serum concentrations of homocysteine in men: a population study. Am J. Clin. Nutr. 80(6), 1574-1578 (2004).

34. Cantoni GL. The role of S-adenosylhomocysteine in the biological utilization of S-adenosylmethionine. Prog. Clin. Biol. Res. 198, 47-65 (1985).

35. Chiang PK, Cantoni GL. Perturbation of biochemical transmethylations by 3-deazaadenosine in vivo. Biochem. Pharmacol. 28(12), 1897-1902 (1979).

36. Hoffman DR, Marion DW, Cornatzer WE, Duerre JA. S-Adenosylmethionine and S-adenosylhomocystein metabolism in isolated rat liver. Effects of L-methionine, L-homocystein and adenosine. J. Biol. Chem. 255(22), 10822-10827 (1980).

37. Crider KS, Yang TP, Berry RJ, Bailey LB. Folate and DNA methylation: a review of molecular mechanisms and the evidence for folate's role. Adv. Nutr. 3(1), 21-38 (2012).

38. Kok DE, Dhonukshe-Rutten RA, Lute $\mathrm{C}$ et al. The effects of long-term daily folic acid and vitamin B12 supplementation on genome-wide DNA methylation in elderly subjects. Clin. Epigenetics 7, 121 (2015).

39. Fenech M, Aitken C, Rinaldi J. Folate, vitamin B12, homocysteine status and DNA damage in young Australian adults. Carcinogenesis 19(7), 1163-1171 (1998).

40. Jung AY, Smulders $Y$, Verhoef $P$ et al. No effect of folic acid supplementation on global DNA methylation in men and women with moderately elevated homocysteine. PLoS ONE 6(9), e24976 (2011).

- There is no effect of folic acid supplementation on DNA methylation in moderately hyperhomocysteinemic subjects.

41. Basten GP, Duthie SJ, Pirie L, Vaughan N, Hill MH, Powers HJ. Sensitivity of markers of DNA stability and DNA repair activity to folate supplementation in healthy volunteers. Br. J. Cancer 94(12), 1942-1947 (2006)

42. Jones PA. Functions of DNA methylation: islands, start sites, gene bodies and beyond. Nat. Rev. Genet. 13(7), 484-492 (2012).

43. Kumar KA, Lalitha A, Pavithra D et al. Maternal dietary folate and/or vitamin B12 restrictions alter body composition (adiposity) and lipid metabolism in Wistar rat offspring. J. Nutr. Biochem. 24(1), 25-31 (2013).

44. Kumar KA, Lalitha A, Reddy U, Chandak GR, Sengupta S, Raghunath M. Chronic maternal vitamin B12 restriction-induced changes in body composition \& glucose metabolism in the Wistar rat offspring are partly correctable by rehabilitation. PLoS ONE 9(11), e112991 (2014).

45. Adaikalakoteswari A, Finer S, Voyias PD et al. Vitamin B12 insufficiency induces cholesterol biosynthesis by limiting s-adenosylmethionine and modulating the methylation of SREBF1 and LDLR genes. Clin. Epigenetics 7, 14 (2015).

46. Dayeh T, Volkov P, Salo $S$ et al. Genome-wide DNA methylation analysis of human pancreatic islets from Type 2 diabetic and nondiabetic donors identifies candidate genes that influence insulin secretion. PLoS Genet. 10(3), e1004160 (2014).

-• Several Type 2 diabetes-associated loci including $F T O$ and $T C F 7 L 2$ show differential methylation in pancreatic islets of diabetic and nondiabetic individuals.

47. Bell CG, Finer S, Lindgren CM et al. Integrated genetic and epigenetic analysis identifies haplotype-specific methylation in the FTO Type 2 diabetes and obesity susceptibility locus. PLoS ONE 5(11), e14040 (2010).

48. Hall E, Volkov P, Dayeh T et al. Sex differences in the genome-wide DNA methylation pattern and impact on gene expression, microRNA levels and insulin secretion in human pancreatic islets. Genome Biol. 15(12), 522 (2014).

49. Fernandez-Valverde SL, Taft RJ, Mattick JS. MicroRNAs in beta-cell biology, insulin resistance, diabetes and its complications. Diabetes 60(7), 1825-1831 (2011)

50. Ross SA, Davis CD. The emerging role of microRNAs and nutrition in modulating health and disease. Annu. Rev. Nutr. 34, 305-336 (2014).

51. Adams AT, Kennedy NA, Hansen R et al. Two-stage genome-wide methylation profiling in childhood-onset Crohn's disease implicates epigenetic alterations at the VMP1/MIR21 and HLA loci. Inflamm. Bowel Dis. 20(10), 1784-1793 (2014).

-. Hypomethylation of specific CpGs (cg16936953, cg12054453, cg01409343 and cg02782634) at the miR21 locus is observed in Crohn's disease and it leads to increased expression of $m i R 21$ in the peripheral blood. 
52. Zhou Y, Park SY, Su J et al. TCF7L2 is a master regulator of insulin production and processing. Hum. Mol. Genet. 23(24), 6419-6431 (2014).

53. Grimm ER, Steinle NI. Genetics of eating behavior: established and emerging concepts. Nutr. Rev. 69(1), 52-60 (2011).

54. Zhou XY, Shibusawa N, Naik K et al. Insulin regulation of hepatic gluconeogenesis through phosphorylation of CREB-binding protein. Nat. Med. 10(6), 633-637 (2004).

55. Qi L, Saberi M, Zmuda E et al. Adipocyte CREB promotes insulin resistance in obesity. Cell Metab. 9(3), 277-286 (2009).

56. Yamauchi T, Oike Y, Kamon J et al. Increased insulin sensitivity despite lipodystrophy in Crebbp heterozygous mice. Nat. Genet. 30(2), 221-226 (2002).

57. Kitada M, Koya D. SIRT1 in Type 2 diabetes: mechanisms and therapeutic potential. Diabetes Metab. J. 37(5), 315-325 (2013).

58. Leenen FA, Muller CP, Turner JD. DNA methylation: conducting the orchestra from exposure to phenotype? Clin. Epigenetics 8, 92 (2016).

59. Anderson OS, Sant KE, Dolinoy DC. Nutrition and epigenetics: an interplay of dietary methyl donors, one-carbon metabolism and DNA methylation. J. Nutr. Biochem. 23(8), 853-859 (2012). 


\title{
Methylation changes and pathways affected in preterm birth: a role for SLC6A3 in neurodevelopment
}

\author{
Ana Arpón 1,2, Fermín I Milagro ${ }^{1,2,3}$, Ana Laja4, Víctor Segura5 ${ }^{5}$, Miguel Sáenz de \\ Pipaón ${ }^{6,7,8}$, José-Ignacio Riezu-Boj $\ddagger 1,2,9$ \& J Alfredo Martínez ${ }^{*} \ddagger, 1,2,3,9,10$ \\ ${ }^{1}$ Department of Nutrition, Food Sciences \& Physiology, Universidad de Navarra, Irunlarrea 1, 31008 Pamplona, Spain \\ ${ }^{2}$ Centre for Nutrition Research, Universidad de Navarra, Irunlarrea 1, 31008 Pamplona, Spain \\ ${ }^{3}$ Spanish Biomedical Research Centre in Physiopathology of Obesity \& Nutrition (CIBERobn), Institute of Health Carlos III, Madrid, \\ Spain \\ ${ }^{4}$ Department of Pharmaceutical \& Health Sciences, Faculty of Pharmacy, CEU San Pablo University, Madrid, Spain \\ ${ }^{5}$ Unit of Bioinformatics, Centre for Applied Medical Research (CIMA), Universidad de Navarra, Pamplona, Spain \\ ${ }^{6}$ Neonatology Department, Hospital Universitario de la Paz, Madrid, Spain \\ ${ }^{7}$ Instituto de Salud Carlos III, Red de Salud Materno Infantil y Desarrollo (SAMID), Madrid, Spain \\ ${ }^{8}$ Department of Pediatrics, Universidad Autónoma de Madrid, Madrid, Spain \\ ${ }^{9}$ Digestive disease and Nutrition group, Navarra Institute for Health Research (IdiSNa), Pamplona, Spain \\ ${ }^{10}$ Madrid Institute of Advanced Studies (IMDEA), IMDEA Food, Madrid, Spain \\ * Author for correspondence: Tel.: +34 948425 600; Fax: +34 948425 740; jalfmtz@unav.es \\ $\ddagger$ These authors share senior authorship
}

\begin{abstract}
Aim: To analyze whether preterm newborns show differences in methylation patterns in comparison to full-term newborns in white blood cells. Patients \& methods: Anthropometrical, biochemical features and methylation levels of preterm newborns $(n=24)$ and full-term newborns $(n=22)$ recruited in La Paz University Hospital (Spain) were assessed at 12 months of gestational age, whereas Bayley Scale of Infant Development was evaluated at 24/36 months. Results: From all the statistically significant CpGs, methylation levels of cg00997378 (SLC6A3 gene) showed the highest differences $(p<0.0001)$, being associated with prematurity risk factors. Conclusion: SLC6A3 methylation, previously related to attentiondeficit/hyperactivity disorder, neuronal function and behavior, might be a potential epigenetic biomarker with value in the early diagnosis and management of neurodevelopmental diseases in newborns.
\end{abstract}

First draft submitted: 3 July 2017; Accepted for publication: 29 September 2017; Published online: 27 November 2017

Keywords: Bayley scale $\bullet$ epigenetics $\bullet$ full-term newborns $\bullet$ peripheral white blood cells $\bullet$ prematurity $\bullet$ preterm newborns • SLC6A3

Despite recent advances in healthcare, preterm births continue to be a major health issue [1]. Preterm newborns (PT) not only exhibit an increased risk of mortality and morbidity in infancy but also the risk of disabilities and developing chronic diseases into adulthood, such as hypertension, Type 2 diabetes, cardiovascular disease and obesity $[1,2]$. Furthermore, PT children have an increased risk of neurodevelopmental and behavioral disorders such as attention-deficit/hyperactivity disorder (ADHD) [1], cerebral palsy or specific learning and behavioral impairments [3]. The underlying molecular mechanisms involved in the higher likelihood of suffering these lifelong effects are still not clear, though epigenetics is probably contributing to a part of this increased risk [4].

Epigenetic mechanisms play a crucial role in fetal development [2,5]. During these stages, epigenetic programming is especially susceptible to environmental factors, including maternal nutrition and health behavior [4,6]. Alterations in the epigenome in these first steps of life can lead to an increased risk of later developing chronic diseases [2].

Epigenetics have been defined as the study of heritable changes that cannot be merely explained through variations in DNA nucleotide sequence but can result in alterations of gene expression [7], providing a connection among genetics, diseases and the environment [8]. DNA methylation is one of the most investigated epigenetic processes in mammals, primarily occurring in CG sites (CpGs) by the addition of a methyl group in the carbon $5^{\prime}$ position

Future Medicine 
of a cytosine adjacent to a guanine [9]. Interestingly, temporal and tissue-specific gene expression is guided by DNA methylation during fetal development and tissue differentiation [1]. Furthermore, DNA methylation has been associated with gestational age $[10,11]$. Once born, DNA methylation is not permanent and may be influenced by drugs, nutrition, lifestyle, etc. [12].

Due to advances in perinatal medicine, the survival of preterm infants has increased considerably in the last years [1]. However, prematurity is accompanied by a risk of adverse neonatal outcomes. Modulation of gene expression through epigenetic mechanisms could play an important role in the development of neurological and metabolic diseases; however, to date, very few studies have been conducted in PT. The aim of this study was to determine epigenetic differences between PT and full-term newborns (FT), which could help to explain the adverse effects associated with prematurity.

\section{Patients \& methods}

\section{Study design \& participants}

This study involved a randomly selected group from a cohort of newborns from the neonatal unit of the La Paz University Hospital in Madrid, Spain, as a convenient sample for exploratory investigation. This group comprises 24 PT and 22 FT, who were controlled postnatally concerning nutritional and environmental conditions during the first 3 years. PT group includes newborns with a gestational age $<34$ weeks and $<1500 \mathrm{~g}$ of body weight, which are classified as very low birth weight newborns, whereas FT had a gestational age $>37$ weeks. Only PT classified as very low birth weight were included since they are at higher risk of stress and nutrition deficit than other PT, which in turn may lead to perinatal complications through epigenetic mechanisms. Exclusion criteria were the presence of congenital diseases, chromosomal abnormalities and short bowel syndrome or others digestive disorders, where absorption of nutrients might be impaired. The local research ethics committee of the La Paz University Hospital approved the study and written informed consent was obtained from the parent(s).

Anthropometrical and body composition measurements including weight, length, head circumference (HC) and fat mass were recorded at 12 months following standardized protocols. A nude weight was obtained in duplicate using a Seca electronic infant scale accurate to the nearest $10 \mathrm{~g}$ (Seca 375, Hamburg, Germany) and length was obtained in duplicate on an infant length board to the nearest $0.1 \mathrm{~cm}$ (Seca 210, Hamburg, Germany). HC was measured to the nearest millimeter by using validated procedures. Fat mass was obtained with dual-energy x-ray absorptiometry following manufacturer's instructions (Lunar-DPX-MD; GE Healthcare, Chalfont St Giles, UK) and analyzed by using infant whole body analysis software (General Electric, Chalfont St Giles, UK).

Biochemical measurements recorded at 12 months included plasma glucose, total cholesterol, insulin, triglycerides, leptin, adiponectin, acylated ghrelin, IL-6 and cortisol, which were measured following validated procedures at the La Paz Hospital. Glucose was immediately quantified from blood samples by enzymatic methods in an autoanalyzer. Insulin was determined by radioimmunoassay (Diagnostic Products Corporation, CA, USA). The homeostatic model assessment of insulin resistance was calculated with the following formula: [glucose $(\mathrm{mmol} / \mathrm{l}) \times$ insulin $(\mathrm{mU} / \mathrm{ml})] / 22.5$. The rest of measurements were obtained from the medical records at the La Paz University Hospital in Madrid. Developmental outcomes were assessed at 24 months of gestational age using the Bayley scale of Infant Development version II (BSID-II) [13] and at 36 months using the BSID version III (BSID-III) [14]. Perinatal complications of PT such as periventricular leukomalacia, retinopathy, sepsis, chronic lung disease and intraventricular hemorrhage were recorded. Maternal and paternal data such as age, height, weight, BMI, educational level, smoking and presence of history of diabetes were collected at the La Paz University Hospital in Madrid.

Nutritional management entailed the following recommendations for PT: a supplement of elemental iron at $2 \mathrm{mg} / \mathrm{kg}$ per day starting at 2 months of age and extending through to 12 months of age; exclusive breastfeeding for a minimum of 4 months but preferably for 6 months, without supplementation after discharge; if human milk was not available, preterm formula was used until 3 months of age. Preterm formula was used only for infants with bronchopulmonary dysplasia. For FT, exclusive breastfeeding was also recommended for a minimum of 4 months but preferable for 6 months, and no iron supplementation was suggested. The iron requirements for toddlers (1-3 years of age) were presumed to be met with naturally iron-rich foods.

DNA extraction \& methylation analysis

All products and reagents were purchased from Sigma-Aldrich (MO, USA) unless otherwise indicated. After overnight fasting, venous blood samples were obtained in ethylenediaminetetraacetic acid (EDTA) tubes at 12 months of age. Blood was transferred to other tubes and red blood lineage was lysed by adding cold water 
$\left(4^{\circ} \mathrm{C}\right)$ up to a total of $50 \mathrm{ml}$ and gently mixing. Samples were centrifuged at $2000 \times \mathrm{g}, 4^{\circ} \mathrm{C}, 20 \mathrm{~min}$. Supernatants were removed and pellets were washed and resuspended with $25 \mathrm{ml}$ of $0.1 \%$ Igepal CA-630 to break membranes and centrifuged at $2000 \times g$ for $20 \mathrm{~min}$ at $4^{\circ} \mathrm{C}$. Supernatants were discarded and pellets (nuclear fraction) were lysed with $3 \mathrm{ml}$ of lysis buffer ( $10 \mathrm{mM}$ Tris, $400 \mathrm{mM} \mathrm{NaCl}$ and $2 \mathrm{mM} \mathrm{Na}_{2}$ EDTA). After this, $600 \mu$ of Proteinase K solution ( $2 \mathrm{mg}$ Proteinase $\mathrm{K}+1 \mathrm{ml}$ of $2 \mathrm{mM} \mathrm{Na}_{2}$ EDTA and 1\% SDS) and $200 \mu \mathrm{l}$ of $10 \%$ SDS were added to samples and they were incubated at $60^{\circ} \mathrm{C}$ for $2 \mathrm{~h}$ or at $37^{\circ} \mathrm{C}$ overnight. Then, samples were tempered at room temperature and proteins were precipitated by vigorous mixing with $1 \mathrm{ml}$ of saturated ammonium acetate solution $(9.6 \mathrm{~mol} / \mathrm{l})$ for $15 \mathrm{~s}$ and resting $10 \mathrm{~min}$ at room temperature. Samples were centrifuged at $2000 \times g$ for $20 \mathrm{~min}$. Supernatants were transferred to other tubes and DNA was precipitated with two volumes of absolute ethanol, recovered with a blunt and curve Pasteur pipette and dissolved in another tube with enough TE buffer $(10 \mathrm{ml}$ Tris- $\mathrm{HCl}[\mathrm{pH} 8.0]$ and $1 \mathrm{mM}$ EDTA [pH 8.0]) to reach a final concentration of $250 \mu \mathrm{g} / \mathrm{ml}$. Isolated DNA of peripheral white blood cells samples were shipped on dry ice to Unidad de Genotipado y Diagnóstico Genético from Fundación Investigación Clínico de Valencia (INCLIVA), where microarray preparation, hybridization and scanning were performed. As previously described by Mansego et al., DNA was quantified using PicoGreen doublestranded DNA Quantification Reagent (Invitrogen, CA, USA). EZ DNA Methylation Kit (Zymo Research, CA, USA) was used to bisulfite modification of $500 \mathrm{mg}$ of genomic DNA according to the manufacturer's protocol [15]. Bisulfite-treated genomic DNA was amplified and hybridized using the Infinium HumanMethylation450 BeadChip (Illumina, CA, USA) and scanned using the Illumina hiScanSQ platform. The intensity of the images was extracted with the GenomeStudio Methylation Software Module following the instructions of the company (v 1.9.0, Illumina).

\section{Treatment of methylation raw data}

Microarray data were normalized (intracell type) using the Subset-quantile Within Array Normalization method to avoid the bias introduced by the Infinium type 2 probe design [16]. After data normalization, the magnitude of batch effects was assessed and corrected using the ComBat normalization method [17,18]. Differences in methylation resulting from differences in cellular heterogeneity were corrected by the Houseman method [19]. Linear Models for Microarray Data [20] for the R statistical software [21] were used to compute a F-statistic adjusted by sex. Raw p-values were adjusted with the Benjamini-Hochberg procedure.

Ingenuity Pathway Analysis

Out of all the significant CpGs obtained after Linear Models for Microarray Data analysis ( $p<0.05$ ), a number of them were selected in order to have an appropriate quantity of $\mathrm{CpGs}$ for obtaining relevant results in the analysis of canonical pathways by the Ingenuity Pathway Analysis (IPA) platform (Qiagen, CA, USA [22]). Due to the high number of tests performed in this study, type I error cannot be discarded. Therefore, in order to avoid this error, a stringent selection of CpGs with an effect size $\leq-4$ or $\geq 4$ and $B>0$ was further implemented. The effect size represents the methylation for the $\mathrm{CpG}$ site. A positive value of effect size indicates hypermethylation of a $\mathrm{CpG}$ site and a negative value indicates hypomethylation. The B-value represents the log odds that the $\mathrm{CpG}$ is differentially methylated. A B-statistic of zero means the probability of the CpG being differentially methylated is equal to the probability that it is not differentially methylated [23]. Relevant gene regulatory networks for significant differentially methylated genes were determined. Network analyses were performed with the IPA's Core Analysis module. Significance values were calculated based on Fisher's exact test. A p $<0.05$ was defined as a statistically significant over-representation of input genes in a given process, based on IPA threshold recommendations.

\section{Statistical analyzes}

Differences in anthropometric and biochemical features of participants as well as food consumption were explored using Student's $t$-test or Mann-Whitney U test, as appropriate. The Shapiro-Wilk test was used to test for normality and the statistically significant level was set at $\mathrm{p}<0.05$.

Correlations (Pearson or Spearman, as convenient) were calculated between CpG methylation and the different anthropometric, biochemical and neurodevelopmental variables. Multivariable regressions considering methylation and gestational age as independent variables were performed for each of the significant variables obtained after correlations.

CpGs with an effect size $\leq-4$ or $\geq 4$ and $B>0$ were represented in a heat map created with the $\mathrm{R}$ software [21] using library gplots and heatmap. 2 function. 


\begin{tabular}{|c|c|c|c|}
\hline Variable & PT $(n=24)$ & $\mathrm{FT}(\mathrm{n}=22)$ & p-value \\
\hline Sex (women) & $12(50)$ & $9(41)$ & 0.541 \\
\hline Gestational age (weeks) $)^{\dagger}$ & $28.23(0.52)$ & $38.87(0.25)$ & $<0.001$ \\
\hline Weight $(\mathrm{g})^{\dagger}$ & $8478(1322)$ & $9826(1368)$ & 0.039 \\
\hline Length $(\mathrm{cm})^{\dagger}$ & $72.85(0.71)$ & $76.24(0.49)$ & $<0.001$ \\
\hline Cephalic perimeter $(\mathrm{cm})^{\dagger}$ & $45.84(0.25)$ & $47.27(0.37)$ & 0.003 \\
\hline Fat mass $(\mathrm{g})^{\dagger}$ & $1890(667)$ & 2555 (939) & 0.279 \\
\hline Plasma glucose $(\mathrm{mg} / \mathrm{dl})^{\dagger}$ & $74.58(1.36)$ & $74.24(1.70)$ & 0.874 \\
\hline Total cholesterol $(\mathrm{mg} / \mathrm{dl})^{\dagger}$ & $159.08(4.67)$ & $144.86(6.05)$ & 0.066 \\
\hline Insulin $(\mathrm{mcU} / \mathrm{ml})^{\dagger}$ & $2.38(0.97)$ & $2.71(1.36)$ & 0.461 \\
\hline Triglycerides $(\mathrm{mg} / \mathrm{dl})^{\dagger}$ & $97.67(6.97)$ & $116.00(13.20)$ & 0.229 \\
\hline Leptin $(n g / l)^{\dagger}$ & $3.52(1.44)$ & $3.80(1.95)$ & 0.768 \\
\hline Adiponectin $(\mu \mathrm{g} / \mathrm{ml})^{\dagger}$ & $22.82(7.65)$ & $23.18(8.13)$ & 0.889 \\
\hline Acylated ghrelin $(\mathrm{pg} / \mathrm{ml})^{\dagger}$ & $65.70(31.15)$ & $77.25(40.38)$ & 0.182 \\
\hline $\mathrm{IL}-6(\mathrm{pg} / \mathrm{ml})^{\dagger}$ & $2.29(2.36)$ & $3.68(3.25)$ & 0.391 \\
\hline Cortisol $(\mu \mathrm{g} / \mathrm{dl})^{\dagger}$ & $12.09(1.16)$ & $11.87(0.78)$ & 0.878 \\
\hline HOMA-IR & $0.66(0.33)$ & $0.51(0.25)$ & 0.284 \\
\hline BSID-II motor ${ }^{\ddagger}$ & $82.78(3.51)$ & $104.09(2.84)$ & $<0.001$ \\
\hline BSID-II mental $\left.\right|^{\ddagger}$ & $92.57(4.88)$ & $107.23(2.89)$ & 0.014 \\
\hline BSID-III cognitive ${ }^{\S}$ & $100.50(10.50)$ & $99.74(5.89)$ & 0.232 \\
\hline BSID-III language ${ }^{\S}$ & $103.70(9.44)$ & $104.79(6.26)$ & 0.425 \\
\hline BSID-III receptive language subscale ${ }^{\S}$ & $11.70(0.22)$ & $11.63(0.38)$ & 0.876 \\
\hline BSID-III expressive language subscale ${ }^{\S}$ & $9.05(1.39)$ & $9.47(1.58)$ & 0.597 \\
\hline BSID-III motor 3 & $94.60(9.95)$ & $96.11(21.27)$ & 0.152 \\
\hline BSID-III fine motor subscale ${ }^{\S}$ & $9.45(0.34)$ & $10.74(0.30)$ & 0.008 \\
\hline BSID-III gross motor subscale ${ }^{\S}$ & $8.70(2.20)$ & $8.42(2.97)$ & 0.432 \\
\hline \multicolumn{4}{|c|}{$\begin{array}{l}\text { Values are represented as mean (SD) except for Sex which is represented as } n(\%) \text {. Values measured at † } 12, \ddagger 24 \text { or }{ }^{\S} 36 \text { months. } \\
\text { p-values were obtained by Student's } t \text {-test, median test or Mann-Whitney's } U \text { test, when appropriate. } p<0.05 \text { was considered significant. } \\
\text { BSID-II: Bayley Scale of Infant Development version II; BSID-III: Bayley Scale of Infant Development version III; FT: Full-term newborns; HOMA-IR: Homeostatic model assessment of insulir } \\
\text { resistance; PT: Preterm newborns; SD: Standard deviation. }\end{array}$} \\
\hline
\end{tabular}

Statistical calculations and graphs were performed using STATA version 12.0 (Stata Corp, TX, USA) and GraphPad Prism 6 (Graph-Pad Software, CA, USA), respectively.

\section{Results}

Preterm newborns showed important changes in anthropometrical, biochemical \& neurodevelopmental features

PT and FT showed expected differences in some anthropometrical, biochemical and neurodevelopmental features. Specifically, statistically significant differences were found in gestational age, weight, length, HC, the BSID-II motor scale, the BSID-II mental scale and the BSID-III fine motor subscale (Table 1). In all cases, the values of PT were lower than FT. Considering brain damage, seven PT showed periventricular leukomalacia and two of them also exhibited moderate-to-severe intraventricular hemorrhage (grades III and IV) as reported (Supplementary Table 1), although only one was considered pathological (BSID-II $<70$ ). Other perinatal complications were retinopathy $(n=7)$, sepsis $(n=6)$ and chronic lung disease $(n=11)$ as reported (Supplementary Table 1$)$. Maternal and paternal data are described in Supplementary Table 1 for PT and Supplementary Table 2 for FT.

The most relevant differentially methylated CpGs were related to nervous system \& general body development

Methylation data were analyzed by comparing the two groups of newborns (PT and FT) and a great number of significant $\mathrm{CpGs}$ were identified. The most relevant $\mathrm{CpGs}$ were selected by $\mathrm{B}>0$ and effect size $\leq-4$ or $\geq 4$, resulting in a total of $317 \mathrm{CpGs}$, corresponding to 232 genes according to the Illumina CG database. These 


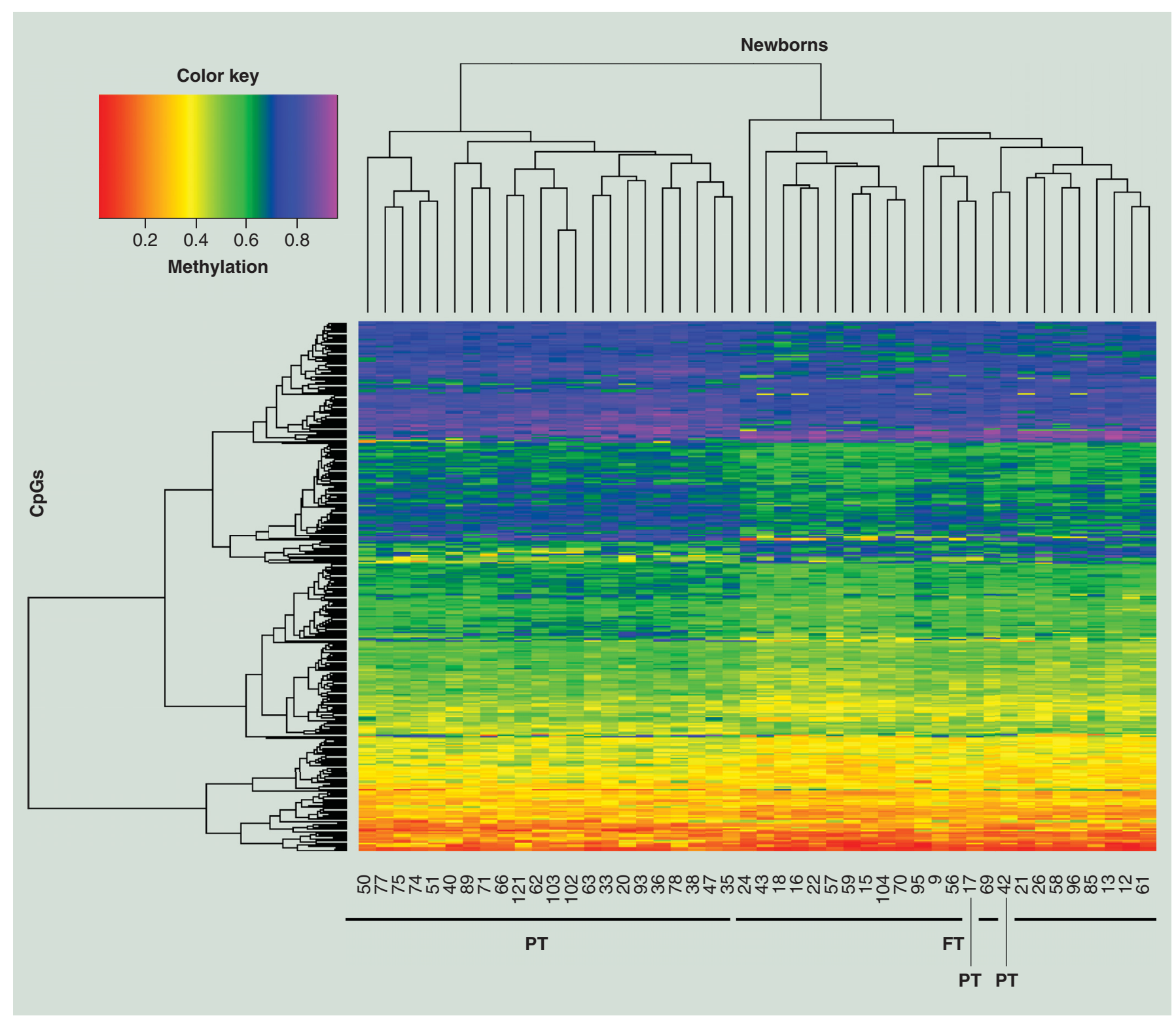

Figure 1. Heatmap of CpGs selected by effect size $\leq-4$ or $\geq 4$ and $B>0$.

selected CpGs separated PT from FT as shown in the clusters, except for two PT that were grouped with FT (Figure 1). When these CpGs were screened by IPA, relevant gene regulatory networks were identified, in which highly significant genes were implicated (Table 2). Most of the identified networks were related to nervous system and general body development, such as neurological disease, cellular development, nervous system development and function, cardiovascular system development and function, tissue development or auditory and vestibular system development and function.

\section{CpG cg00997378 showed the largest methylation differences between PT \& FT}

After this preliminary analysis, a search of specific CpGs with interesting functions was performed. The CpG cg00997378 was chosen as it showed the largest methylation differences between the two groups of newborns $(\mathrm{p}$-value $=3.87 \mathrm{E}-11, \mathrm{p}$-value adjusted with Benjamini-Hochberg $=1.51 \mathrm{E}-05, \mathrm{~B}=14.63)$ as reported (Figure 2). The CpG cg00997378 was located in 5' UTR of the corresponding gene named Solute Carrier Family 6 Member 3 (SLC6A3) in chromosome 5 (position: 1444260). 
Table 2. Networks from Ingenuity Pathway Analysis obtained with a selection of CpGs with effect size $\leq-4$ or $\geq 4$ and B $>0$.

\section{Top diseases and functions}

Cancer, dermatological diseases

and conditions, organismal injury

and abnormalities

\section{Score Focus molecules Molecules in network}

22

AGBL1, BSN, C2orf54, CACNB1, CERS1, CERS4, DES, FBXL22, GDF1, GIMAP5, HHIPL1, HLA-DQB2, HSPA7, KLHL35, miR-3689d (and other miRNAs w/seed GGAGGUG), miR-6081 (miRNAs w/seed GGAGCAG), miR-6742-3p (miRNAs w/seed CCUGGGU), OC90, PCDHA1, PCDHA2, PCDHA3, PCDHA4, PCDHA6, PCDHA7, PCDHA8, PCNX2, SOGA3, TEAD3, TMEM53, TP63, TRIML2, UBE2G2, UNC119B, voltage-gated calcium channel, WNK2

\begin{tabular}{|c|c|c|c|}
\hline $\begin{array}{l}\text { Molecular transport, hereditary } \\
\text { disorder, metabolic disease }\end{array}$ & 33 & 19 & $\begin{array}{l}26 \text { s Proteasome, ABCC1, ARHGAP27, CDH22, Ck2, DMTN, EP400, estrogen receptor, F actin, FOXR1, } \\
\text { GTPase, HDAC, hemoglobin, HIF3A, HISTONE, Histone h3, Histone h4, HLCS, Hsp70, Hsp90, ISL1, KLF1, } \\
\text { MAD1L1, NFkB (complex), Nr1h, PCDHA5, PREX1, Proinsulin, PTPRN2, RAB3C, SLC2A12, SLC6A19, TAP1, } \\
\text { TRIM6, Ubiquitin }\end{array}$ \\
\hline $\begin{array}{l}\text { Cardiovascular system } \\
\text { development and function, } \\
\text { organ development and nervous }\end{array}$ & 26 & 16 & $\begin{array}{l}\text { ADCY, ADRB, ALP, AMPK, ARHGAP35, atrial natriuretic peptide, CACNA1D, CAT, Cg, collagen type I, } \\
\text { DDAH1, DIO2, ERK1/2, FSH, GNAI1, HRC, IFIT3, INSL3,KCNQ1, LDL, Lh, MAST1, NOS3, p70 S6k, PDGF } \\
\text { BB, Pkg, PMAIP1, PP2A, PPP2R2C, PRKAA, PRLR, SLC6A3, Sod, Spectrin, TSH }\end{array}$ \\
\hline
\end{tabular}

organ development and nervous

system development and

function

Cellular development, nervous $\quad 26 \quad 16$

system development and

function, tissue development

ALDH3B2, CAV3, CYP26B1, CYP4F11, DNASE1L1, FBXW8, GJD3, GXYLT2, H2AFB3 (includes others) HOXB5, JPH2, KDM3B, LGR6, LRRC15, MDGA1, miR-105-3p (miRNAs w/seed CGGAUGU), miR-1471 (miRNAs w/seed CCCGCGU), miR-3621 (miRNAs w/seed GCGGGUC), miR-4723-5p (and other miRNAs w/seed GGGGGAG), miR-572 (miRNAs w/seed UCCGCUC), miR-6070 (miRNAs w/seed CGGUUCC), miR-6125 (miRNAs w/seed CGGAAGG), miR-6741-3p (miRNAs w/seed CGGCUCU), NANOS2, NLGN2, NOL10, NPAS3, P2RY8, PALM, POT1, POU6F2, SLC8A1, TMEM151B, TMEM63C, ZNF512B

\begin{tabular}{|c|c|c|c|}
\hline $\begin{array}{l}\text { Developmental disorder, } \\
\text { hereditary disorder, ophthalmic } \\
\text { disease }\end{array}$ & 26 & 16 & $\begin{array}{l}\text { ADAMTS17, ADAMTS19, ANKRD30B, AZIN2, C10orf71, DENND4B, DNAJB13, FBXL6, FCAR, GALNT10, } \\
\text { GLI4, IFNLR1, IRX6, miR-6752-3p (miRNAs w/seed CCCUGCC), miR-6756-5p (and other miRNAs w/seed } \\
\text { GGGUGGG), miR-6880-5p (miRNAs w/seed GGUGGAG), MLLT1, MOGAT3, NEMP2, OR2F1, OR2F2, } \\
\text { P2RX5, PI16, PRPF40B, PRR34, PRSS36, PRX, RBAK-RBAKDN, REEP6, SNED1, SNX15, TMPRSS9, ZBTB38, } \\
\text { ZDHHC24, ZNF554 }\end{array}$ \\
\hline $\begin{array}{l}\text { Cellular movement, cancer, } \\
\text { organismal injury and } \\
\text { abnormalities }\end{array}$ & 24 & 15 & $\begin{array}{l}\text { ABR, Akt, ALK, ALPI, ANGPTL2, Ap1, c-Src, calcineurin protein(s), calpain, COL5A3, collagen(s), G } \\
\text { protein alphai, GP6, IgA, IgE, IgG, immunoglobulin, ITGB3, MHC, mir-15, MYO16, NFAT (complex), } \\
\text { NFATC1, NR4A2, P38 MAPK, Pdgf (complex), PDGFD, PI3K (complex), PLC- } \gamma \text {, Rac, SEMA4B, SLFN12L, } \\
\text { SYK/ZAP, TGF- } \beta \text {, TNFSF8 }\end{array}$ \\
\hline $\begin{array}{l}\text { Cell death and survival, skeletal } \\
\text { and muscular disorders, cancer }\end{array}$ & 24 & 15 & $\begin{array}{l}\text { Actin, ARHGEF17, caspase, CBFA2T3, CD3, Creb, DLC1, ERK, focal adhesion kinase, GNMT, Gpcr, Gsk3, } \\
\text { IGF2, IL12 (complex), insulin, IFN- } \alpha \text {, Jnk, Mapk, MAPK4, MCF2L, NCF4, NRXN1, NXPH3, Pka, Pkc(s), } \\
\text { RABEP1, Ras homolog, SH3BP5, SRC (family), TCR, TNF (family), TP73, TRIM6-TRIM34, TSPAN9, VEGF }\end{array}$ \\
\hline $\begin{array}{l}\text { Cell-to-cell signaling and } \\
\text { interaction, cellular assembly and } \\
\text { organization and cellular } \\
\text { development }\end{array}$ & 22 & 14 & $\begin{array}{l}\text { AP2A2, APP, BEND2, CALML4, CCZ1/CCZ1B, DENND1C, EBF4, FANCM, GLB1L, HERC6, HIGD2A, KCNAB3, } \\
\text { KIAA1217, KIAA1841, KIF1A, KIFC3, KNTC1, LENG1, LMCD1, MAP6, miR-8056 (miRNAs w/seed } \\
\text { GUGGAUU), Na-k-atpase, PRRC1, PRSS3, RALGPS1, RASAL1, RP2, RSRC2, SIX2, SPATS2, TBCD, TUBA8, } \\
\text { TUBB6, ZCCHC12, ZNF483 }\end{array}$ \\
\hline $\begin{array}{l}\text { Cell death and survival, auditory } \\
\text { and vestibular system } \\
\text { development and function, } \\
\text { cellular growth and proliferation }\end{array}$ & 20 & 13 & $\begin{array}{l}\text { AFAP1, ARHGAP25, C2orf27A/C2orf27B, CTNNB1, CTNN } \beta \text {-LEF1, FGF20, FGF18/20, HILPDA, HLA-DQA2, } \\
\text { HRNR, IL7R, KCNQ1OT1, LYNX1, miR-4765 (miRNAs w/seed GAGUGAU), miR-6509-5p (miRNAs w/seed } \\
\text { UUAGGUA), MYC, NFKBIA, NLRP14, NOM1, PCBD1, Pdlim3, RMST, Scf TrCP beta, SOX2, Sprr1b, } \\
\text { Sprr2a1/Sprr2a2, Sprr2b, Sprr2e, Sprr2f, Sprr2g, Sprr2i, Sprr2k, TCF4-CTNN } \beta \text {, TLL2, } \\
\beta \text {-catenin-BCL9-PYGO-TCF/LEF }\end{array}$ \\
\hline $\begin{array}{l}\text { Hereditary disorder, neurological } \\
\text { disease, organismal injury and } \\
\text { abnormalities }\end{array}$ & 18 & 12 & $\begin{array}{l}\text { 3',5'-cyclic-GMP phosphodiesterase, ACKR1, ADORA3, AHNAK, DHRS7, DRD3, DRD4, EGFR ligand, } \\
\text { endothelin receptor, ERBB2, ERICH1, EWSR1, FAM118A, G protein alpha, G-protein gamma, GLRX3, } \\
\text { GNAI1, LCE2C/LCE2D, mGluR, miR-708-3p (miRNAs w/seed AACUAGA), MTNR1A, MTNR1B, OBSCN, } \\
\text { PCNA, PKD1L2, POLI, PTGER1, RGS20, RNA polymerase II, SMAD4, SMC4, SUMO-Ubc9 E2, TTC32, YY1, } \\
\text { ZNF8 }\end{array}$ \\
\hline $\begin{array}{l}\text { Dermatological diseases and } \\
\text { conditions, hereditary disorder, } \\
\text { immunological disease }\end{array}$ & 10 & 8 & $\begin{array}{l}\text { ADGRE2, ADGRE3, CELSR1, CFAP74, CPPED1, ELAC2, FFAR3, fibrin, GADD45GIP1, gamma tubulin, } \\
\text { GTPBP10, HTR1E, LBX2, MCM8, miR-877-5p (and other miRNAs w/seed UAGAGGA), miR-939-3p } \\
\text { (miRNAs w/seed CCUGGGC), MLNR, MMP16, MRPL17, MRPL47, MRPL53, MRPL55, MRPS30, Mt-mmp, } \\
\text { NDUFB8, PGA5 (includes others), Pik3r, RB1, SLC12A4, SLC16A13, SLC52A2, TGM2, transglutaminase, } \\
\text { WFDC10A, ZNF566 }\end{array}$ \\
\hline
\end{tabular}

Methylation changes in cg00997378 were associated with prematurity risk factors

The methylation level of cg00997378 in PT was significantly higher than in FT (Figure 3). The association between methylation and gestational age was statistically significant (Figure 4A). There was also a significant negative association between methylation and some studied variables such as triglycerides, BSID-II motor and 


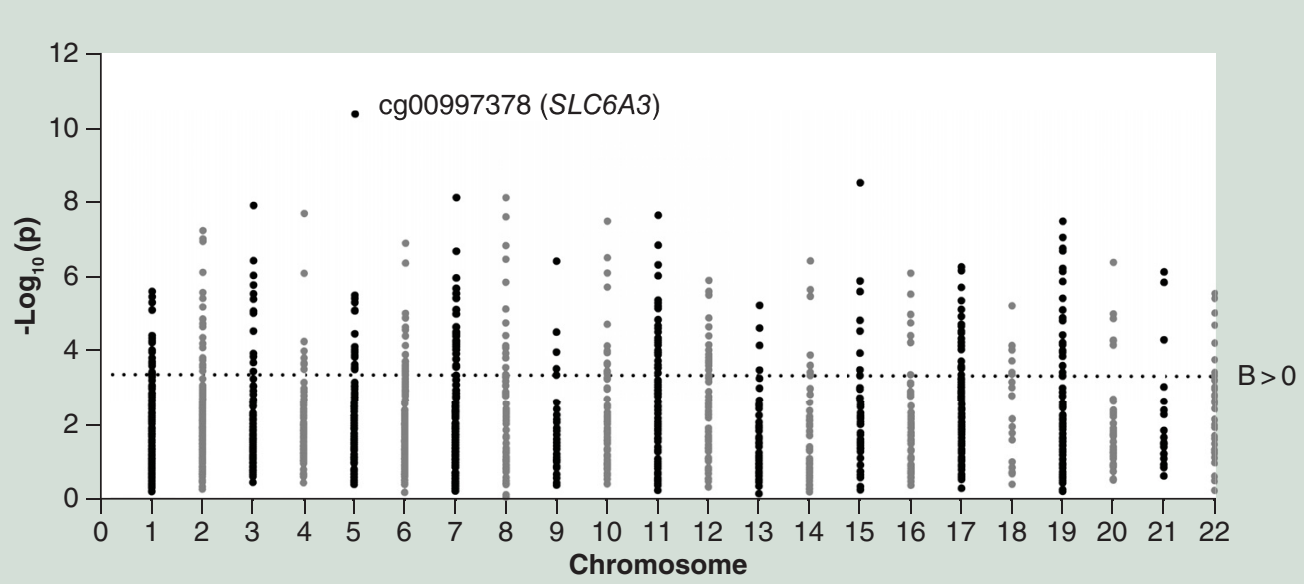

Figure 2. Manhattan plot of CpGs selected by effect size $\leq-\mathbf{4}$ or $\geq \mathbf{4}$ in each chromosome. Points above the horizontal line are $B>0(-\log 10(p)=3.40)$.

Figure 3. Differences in methylation of cg00997378 (SLC6A3) between preterm and full-term newborns. Lines in the distribution represent mean and SD. Two-tailed $p$-value was calculated using Student's $t$-test. $p<0.05$ was considered significant.

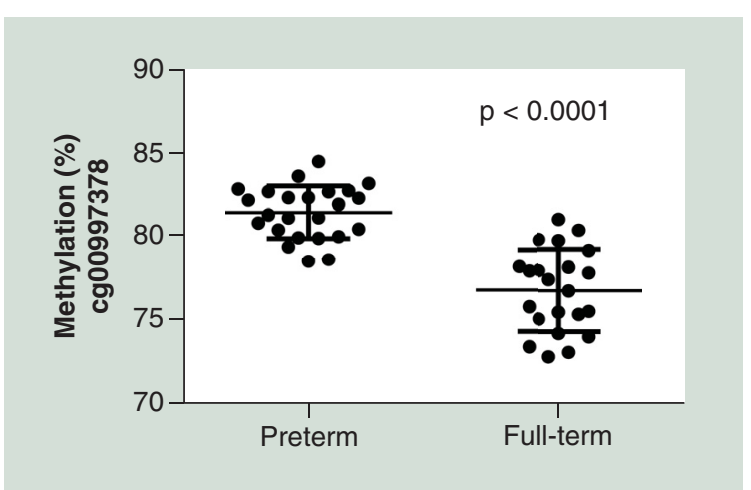

Table 3. Regression values (coefficient and p-value) for cg00997378 methylation in relation to different descriptive variables, adjusted by sex.

\begin{tabular}{|c|c|c|c|c|c|c|c|c|}
\hline \multirow[t]{3}{*}{ Variables } & \multicolumn{4}{|c|}{ Simple linear regression } & \multicolumn{4}{|c|}{ Multiple linear regression } \\
\hline & \multicolumn{2}{|c|}{ Methylation } & \multicolumn{2}{|c|}{ Gestational age } & \multicolumn{2}{|c|}{ Methylation } & \multicolumn{2}{|c|}{ Gestational age } \\
\hline & Coefficient & p-value & Coefficient & p-value & Coefficient & p-value & Coefficient & p-value \\
\hline $\begin{array}{l}\text { Triglycerides } \\
(\mathrm{mg} / \mathrm{dl})\end{array}$ & -6.74 & 0.003 & 1.37 & 0.290 & -11.65 & 0.001 & -3.52 & 0.050 \\
\hline BSID-II motor & -3.25 & $<0.001$ & 2.11 & $<0.001$ & -0.91 & 0.387 & 1.73 & 0.005 \\
\hline BSID-II mental & -2.70 & 0.005 & 1.38 & 0.010 & -1.90 & 0.177 & 0.59 & 0.443 \\
\hline
\end{tabular}

BSID-II mental (Figure 4B-D), displaying r-coefficients of $-0.44,-0.55$ and -0.44 , respectively, corresponding to p-values of 0.0026, 0.0001 and 0.0028 , respectively.

The methylation level of $\operatorname{cg} 00997378$ was associated with descriptive clinical variables that were different between PT and FT as explained before (Table 1 \& Figure 4). Simple and multiple linear regressions adjusted by sex were performed between each one of these measurements as dependent variables and methylation or/and gestational age as independent variables (Table 3) in order to elucidate whether these associations were due to changes in methylation or in gestational age. When comparing simple versus multiple linear regressions results, triglycerides showed a significantly association with cg00997378 methylation in both types of regression, but not with gestational age. Furthermore, the BSID-II motor score showed a significant association with both cg00997378 methylation 


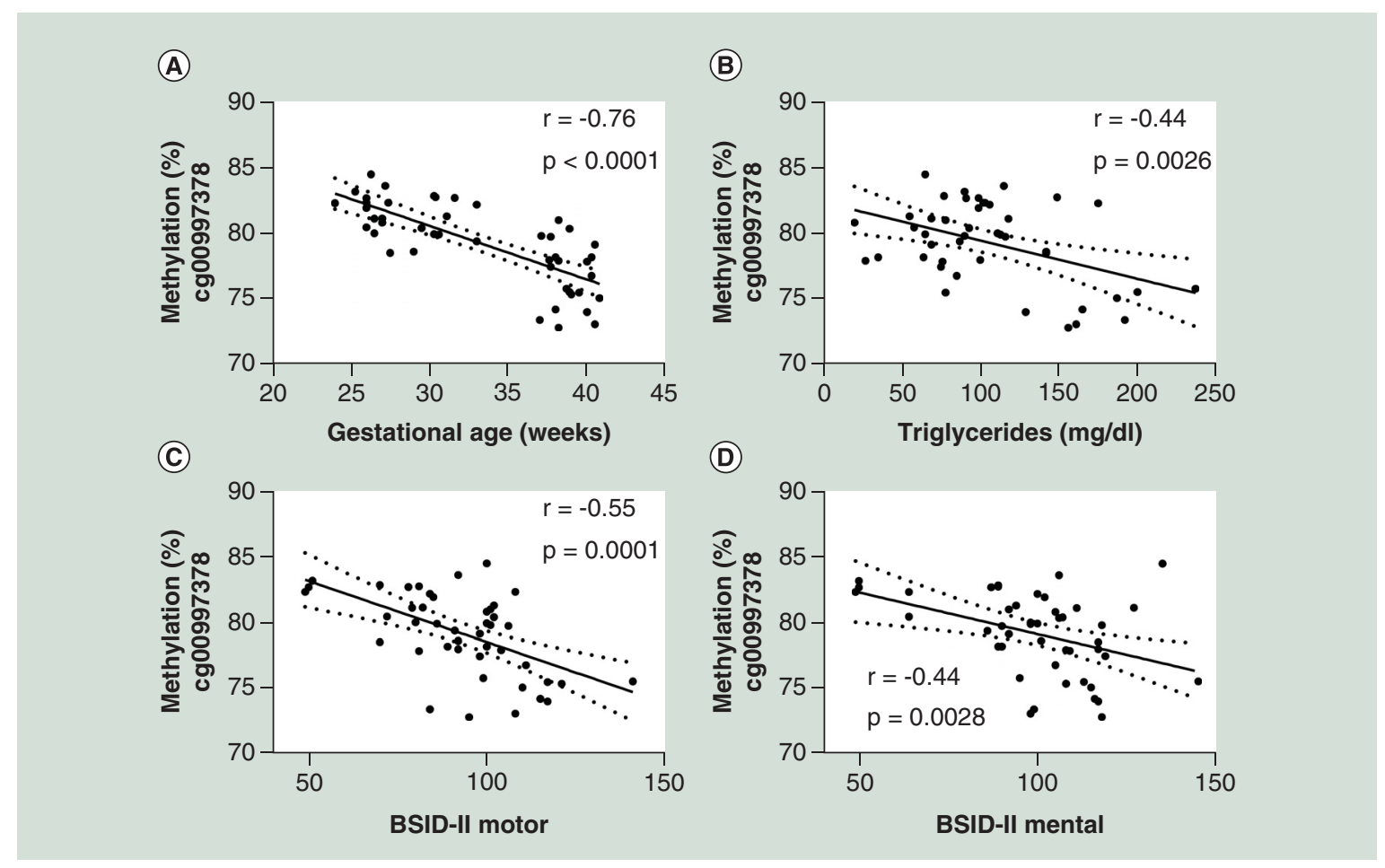

Figure 4. Statistically significant correlation graphs representing the association between methylation of cg00997378 (SLC6A3) with descriptive variables. Dot lines on both sides of the solid line (linear regression for correlation) represent $95 \%$ confidence band. Correlation tests used were Pearson or Spearman, when appropriate. BSID-II: Bayley scale of infant development version II.

and gestational age in the simple linear regression model, but it remained significant for gestational age only in the multiple linear regression model. In the case of the BSID-II mental score, methylation and gestational age were significantly associated with this score in simple linear regressions. However, multiple linear regression revealed no association with either of them. The coefficients for methylation changed from -2.70 to -1.90 , while the coefficient for gestational age changed from 1.38 to 0.59 .

\section{Discussion}

The present study demonstrates that PT and FT exhibit differences in the methylation patterns of a number of genes in peripheral white blood cells, mainly related to nervous system functions and general body development. The CpG with the largest methylation difference between both groups was cg00997378 (SLC6A3 gene). In this study, SLC6A3 methylation has been associated with triglycerides, BSID-II motor and BSID-II mental scores. Furthermore, multiple linear regression analysis suggest a possible influence of $S L C 6 A 3$ methylation and gestational age on neurodevelopment assessed by BSID-II mental scale and a relation between SLC6A3 methylation and triglyceride levels.

Prematurity involves being born before being physically ready for autonomous life [24]. Indeed, this study showed differences between PT and FT in physical and neurodevelopmental characteristics. This developmental immaturity can lead to an increased risk of suffering health problems later in life such as intellectual impairment, cerebral palsy, cardiovascular diseases, obesity, chronic lung disease, vision and hearing loss among others $[1,3]$. Indeed, one of the PT suffered from pathological brain damage since it exhibit periventricular leukomalacia [25], grade IV intraventricular hemorrhage [26] and BSID-II $<70$ [27].

Maternal risk factors can contribute to preterm delivery [28] and changes in the offspring methylation [4]. Spontaneous preterm birth is influenced by age at pregnancy, pregnancy spacing, multiple pregnancies, infections, underlying maternal chronic medical conditions, nutritional factors, lifestyle (alcohol and smoking) and physiological status [28]. Methylation in the offspring is also affected by nutritional factors, lifestyle or stress [4]. Some PT mothers were smokers, reported diabetes during pregnancy and they had a wide range of BMIs (from 17.6 to 
$32.4 \mathrm{~kg} / \mathrm{m}^{2}$ ). However, some FT mothers also exhibited these features. Spontaneous preterm birth is a multifactorial process; therefore, these factor risks might have played a role in PT mothers and in the offspring methylation, whereas this may not be the case in FT birth.

DNA methylation has been associated with gestational age $[10,11]$. In fact, previous studies have demonstrated methylation differences between PT and FT in several genes. For instance, in comparison with FT, lower methylation levels on the IGF2 in PT have been reported in buccal samples [29]. This gene also showed methylation differences between PT and FT in the current study (cg15168906 and cg04057455). Moreover, Kantake et al. described that glucocorticoid receptor promoter methylation at postnatal day 4 was larger in PT than in FT in peripheral white blood cells [30]. Another study showed larger methylation levels of $M C 4 R$ and $H N F 4 \alpha$ in cord blood samples from PT than in those from FT [31]. Neural function-related genes were also reported to be differentially methylated between PT and FT in saliva samples [32]. Thus, the differences in methylation may be associated with the adverse effects that PT may manifest in their development.

In the current study, PT and FT evidenced methylation differences in more than 300 relevant CpGs (effect size $\leq-4$ or $\geq 4$ and $\mathrm{B}>0$ ). These genes are involved in several pathways, most of them related to general body development, including nervous system development and function, cardiovascular system development and function, tissue development or auditory and vestibular system development and function.

The CpG cg00997378 showed the largest differences between both PT and FT. This CpG site is strongly associated with active chromatin sites including DNAse hypersensitivity sites and activates histone $\mathrm{H} 3 \mathrm{k} 27 \mathrm{ac}$ in a wide range of cell lines (USCS Genome Browser). The cg00997378 is located at 5'UTR of SLC6A3 according to the Illumina CG database, possibly affecting its expression. Actually, SLC6A3 encodes a dopamine transporter, belonging to the sodium- and chloride-dependent neurotransmitter transporter family [33].

Interestingly, SLC6A3 methylation showed an association with triglycerides, the BSID-II motor and BSID-II mental scales. When multiple linear regression analyses were performed for these variables, p-values were significant with methylation for the variable triglycerides and with gestational age for the BSID-II motor scale. However, simple linear regressions were significant with both variables for the BSID-II motor scale and only with methylation in the case of triglycerides. Intriguingly, both methylation and gestational age seemed to have an effect on the BSID-II mental scale since both coefficients in multiple linear regression were decreased $30 \%$ for methylation and $57 \%$ for gestational age), while both variables were significantly associated in simple linear regressions. Therefore, it is likely that SLC6A3 methylation is related to triglyceride levels and neurodevelopment.

On one hand, the dopaminergic system is involved in food reward, which regulates food intake [34]. SLC6A3 (dopamine transporter DAT-1) methylation changes may be related to triglyceride levels, as they directly affect cognitive and reward processes, contributing to obesity [35], which has been described as a prematurity risk [1]. On the other hand, dopamine has been intimately related to neurological and behavioral disorders such as Parkinson [36], Tourette syndrome [37], depression [38], behavioral impairments [39] and specially, ADHD. In particular, ADHD patients seem to have a reduced cortical dopamine or dopaminergic dysregulation [40,41]. In addition, a low score on the Bayley Mental Scale has been associated with ADHD [42]. Therefore, SLC6A3 methylation may have a role in neurodevelopment, which may lead to neurologic differences between PT and FT, such as future adverse effects of prematurity-like behavioral problems and ADHD [1].

Methylation changes in $S L C 6 A 3$ have been previously described, since it has high sensitivity to epigenetic regulation [43]. For instance, SLC6A3 methylation has been correlated with age within ADHD patients [44] and involved in the modulation of the response to methylphenidate treatment in ADHD [40]. On the other hand, Vucetic et al. demonstrated that maternal consumption of a high-fat diet can change DNA methylation of the offspring in association with long-term alterations in gene expression (SLC6A3) and behavior (preference for palatable foods) [45]. Furthermore, methylation of this gene has been found to be altered by alcohol exposure during pregnancy [46].

SLC6A3 appeared in IPA networks related to cardiovascular system development and function, organ development and nervous system development and function. Among the genes of this network, there were 14 that appeared differentially methylated between PT and FT when using the selection criteria by B $>0$ and effect size $\leq-4$ or $\geq 4$. Interestingly, 13 of them were also associated with BSID scores (Supplementary Table 3). The fact that there were several differentially methylated genes involved in those pathways and related to neurodevelopment suggests an interconnection among them. In addition, other networks related to general body development involving differentially methylated genes were identified in IPA analysis. Thus, methylation was not singular since several genes 
seemed to be affected, probably creating a network of changes in gene expression that could influence the development of the newborn. Indeed, the finding that methylation of several genes involved in neurodevelopment were conjointly affected offers a unique perspective to understand our hypothesis that epigenetic signature is implicated in gene expression and function.

The present investigation was not devoid of limitations such as the sample size, which was relatively low, increasing the probability for type II error. However, the sample involves a highly specific population. In addition, peripheral white blood cells were used, which are not part of a tissue related to neurodevelopment. However, it has been demonstrated that these cells can act as a surrogate for other tissues that are less accessible but clinically important, such as the brain $[47,48]$.

Further analysis of methylation changes in time would be interesting in order to determine if the differences are maintained or even other variations appear. In addition, investigating mRNA levels would also allow to elucidate whether methylation changes were affecting the expression of $S L C 6 A 3$, unfortunately no samples were available. The use of alternative techniques and replication of the results in other populations would allow validating the present results. However, SLC6A4, paralog of $S L C 6 A 3$ that encodes for a serotonin receptor, evidenced a similar trend when measured by pyrosequencing [49]. Finally, the cross-sectional feature of this research does not allow to set up causal inferences, neither consequences, but it appears that gestational age is important when interpreting the experimental outcomes in relation to $S L C 6 A 3$ methylation changes.

As a conclusion, this study shows that prematurity could contribute to differential methylation in peripheral white blood cells. Specifically, important methylation changes occur in SLC6A3, a gene that has been previously related to ADHD. Therefore, some of the prematurity adverse effects such as neurodevelopmental impairment could be mediated by epigenetic modifications. SLC6A3 might be a potential epigenetic biomarker with value in the early diagnosis of neurological diseases, in order to identify susceptible individuals and develop preventive and therapeutic measures. Further studies are necessary to confirm the interaction between gestational age as a cause of the changes or a causal role for SLC6A3 and genes related with this pathologic pathway.

Summary points

- This group of preterm newborns showed lower values of weight, length, cephalic perimeter, Bayley Scale of Infant Development (BSID) version II motor and mental scales and version III fine motor subscale.

- More than 300 relevant CpGs showed a differential methylation pattern between both groups of newborns in peripheral white blood cells.

- Those CpGs were related to nervous system and general body development.

- cg00997378 showed the largest methylation changes between both groups of newborns.

- cg00997378 methylation level is negatively associated with triglyceride levels and the BSID version II motor and mental scales.

- The BSID version II motor scale is mainly influenced by gestational age, whereas triglycerides by cg00997378 methylation. The BSID version II mental scale appears to be influenced by both gestational age and cg00997378 methylation.

- Prematurity could contribute to differential methylation pattern in peripheral white blood cells.

- Some of the prematurity adverse effects such as neurodevelopmental impairment may be mediated by epigenetic modifications in genes, such as SLC6A3.

Supplementary data

To view the supplementary data that accompany this paper please visit the journal website at: www.futuremedicine.com/doi/full/10.2217/epi-2017-0082

\section{Acknowledgements}

The authors are grateful to E Almiron-Roig (visiting scientist at MRC Elsie Widdowson Laboratory, Cambridge, UK) for reviewing the manuscript prior to submission.

Financial \& competing interests disclosure

Authors are very grateful to CIBERobn (CB12/03/30002 to JA Martínez) and MINECO (AGL 2013-45554-R to JA Martínez and FI Milagro; FIS PI041631 and PI16/00606 to M Sáenz de Pipaón) for financial help and scientific support. A Arpón was supported by 
a 'Formación de Profesorado Universitario' fellow from Ministerio de Educación, Cultura y Deporte (FPU15/02790). The authors have no other relevant affiliations or financial involvement with any organization or entity with a financial interest in or financial conflict with the subject matter or materials discussed in the manuscript apart from those disclosed.

No writing assistance was utilized in the production of this manuscript.

Ethical conduct of research

The local research ethics committee of the La Paz University Hospital approved the study and written informed consent was obtained from the parent(s).

\section{References}

Papers of special note have been highlighted as: $\bullet$ of interest; $\bullet \bullet$ of considerable interest

1. Parets SE, Bedient CE, Menon R, Smith AK. Preterm birth and its long-term effects: methylation to mechanisms. Biology (Basel) 3(3), 498-513 (2014).

-. An overview of the relationship between prematurity and DNA methylation.

2. Agha G, Hajj H, Rifas-Shiman SL et al. Birth weight-for-gestational age is associated with DNA methylation at birth and in childhood. Clin. Epigenetics 8, 118 (2016).

3. Blencowe H, Lee AC, Cousens S et al. Preterm birth-associated neurodevelopmental impairment estimates at regional and global levels for 2010. Pediatr. Res. 74(Suppl. 1), 17-34 (2013).

- A wide summary of neurodevelopmental impairments associated with prematurity.

4. Knight AK, Smith AK. Epigenetic biomarkers of preterm birth and its risk factors. Genes (Basel) 7(4), pii: E15 (2016) (Epub ahead of print).

-. A review of the relationship between environmental risk factors and DNA methylation in preterm newborns.

5. Casanello P, Krause BJ, Castro-Rodriguez JA, Uauy R. Fetal programming of chronic diseases: current concepts and epigenetics. Rev. Chil. Pediatr. 86(3), 135-137 (2015).

6. Geraghty AA, Lindsay KL, Alberdi G, McAuliffe FM, Gibney ER. Nutrition during pregnancy impacts offspring's epigenetic statu evidence from human and animal studies. Nutr. Metab. Insights 8(Suppl. 1), 41-47 (2015).

7. Rozek LS, Dolinoy DC, Sartor MA, Omenn GS. Epigenetics: relevance and implications for public health. Annu. Rev. Public Health 35, 105-122 (2014).

8. Corella D, Ordovás JM. Biomarkers: background, classification and guidelines for applications in nutritional epidemiology. Nutr. Hosp. 31(3), 177-188 (2015).

9. Tammen SA, Friso S, Choi SW. Epigenetics: the link between nature and nurture. Mol. Aspects Med. 34(4), 753-764 (2013).

10. Parets SE, Conneely KN, Kilaru V et al. Fetal DNA methylation associates with early spontaneous preterm birth and gestational age. PLoS ONE 8(6), e67489 (2013).

11. Schroeder JW, Conneely KN, Cubells JC et al. Neonatal DNA methylation patterns associate with gestational age. Epigenetics 6(12), 1498-1504 (2011).

- Association between gestational age and DNA methylation in some genes from umbilical cord blood samples.

12. Lillycrop KA, Burdge GC. Environmental challenge, epigenetic plasticity and the induction of altered phenotypes in mammals. Epigenomics 6(6), 623-636 (2014).

13. Bayley N. Bayley Scales of Infant Development. Psychological Corporation, TX, USA (1993).

14. Bayley N. Bayley Scales of Infant and Toddler Development. Harcourt Assessment, Inc., TX, USA (2006).

15. Mansego ML, Milagro FI, Zulet MA, Moreno-Aliaga MJ, Martinez JA. Differential DNA methylation in relation to age and health risks of obesity. Int. J. Mol. Sci. 16(8), 16816-16832 (2015).

16. Maksimovic J, Gordon L, Oshlack A. SWAN: subset-quantile within array normalization for illumina infinium HumanMethylation450 BeadChips. Genome Biol. 13(6), R44 (2012).

17. Teschendorff AE, Zhuang J, Widschwendter M. Independent surrogate variable analysis to deconvolve confounding factors in large-scale microarray profiling studies. Bioinformatics 27(11), 1496-1505 (2011).

18. Johnson WE, Li C, Rabinovic A. Adjusting batch effects in microarray expression data using empirical Bayes methods. Biostatistics 8(1), 118-127 (2007).

19. Houseman EA, Accomando WP, Koestler DC et al. DNA methylation arrays as surrogate measures of cell mixture distribution. BMC Bioinformatics 13, 86 (2012).

20. Smyth GK. LIMMA: linear models for microarray data. In: Bioinformatics and Computational Biology Solutions Using R and Bioconductor. Gentleman R, Carey VJ, Huber W, Irizarry RA, Dudoit S (Eds). Springer, NY, USA, 397-420 (2005).

21. Team R. RStudio: integrated development for R. www.rstudio.com/ 
22. Qiagen. www.ingenuity.com

23. Martino D, Saffery R. Characteristics of DNA methylation and gene expression in regulatory features on the Infinium 450k Beadchip. bioRxiv doi:10.1101/032862 (2015) (Epub ahead of print).

24. Stoll BJ, Hansen NI, Bell EF et al. Trends in care practices, morbidity, and mortality of extremely preterm neonates, 1993-2012. JAMA 314(10), 1039-1051 (2015).

25. Deng W, Pleasure J, Pleasure D. Progress in periventricular leukomalacia. Arch. Neurol. 65(10), 1291-1295 (2008).

26. Bolisetty $\mathrm{S}$, Dhawan A, Abdel-Latif $\mathrm{M}$ et al. Intraventricular hemorrhage and neurodevelopmental outcomes in extreme preterm infants. Pediatrics 133(1), 55-62 (2014).

27. Jary S, Whitelaw A, Walloe L, Thoresen M. Comparison of Bayley-2 and Bayley-3 scores at 18 months in term infants following neonatal encephalopathy and therapeutic hypothermia. Dev. Med. Child Neurol. 55(11), 1053-1059 (2013).

28. Blencowe H, Cousens S, Chou D et al. Born too soon: the global epidemiology of 15 million preterm births. Reprod. Health 10 (Suppl. 1), S2 (2013).

29. Piyasena C, Cartier J, Khulan B et al. Dynamics of DNA methylation at IGF2 in preterm and term infants during the first year of life: an observational study. Lancet 385(Suppl. 1), S81 (2015).

30. Kantake M, Yoshitake H, Ishikawa H, Araki Y, Shimizu T. Postnatal epigenetic modification of glucocorticoid receptor gene in preterm infants: a prospective cohort study. BMJ Open 4(7), e005318 (2014).

31. Kwon EJ, Lee HA, You YA et al. DNA methylations of MC4R and HNF4alpha are associated with increased triglyceride levels in cord blood of preterm infants. Medicine (Baltimore) 95(35), e4590 (2016).

32. Sparrow S, Manning JR, Cartier J et al. Epigenomic profiling of preterm infants reveals DNA methylation differences at sites associated with neural function. Transl. Psychiatry 6, e716 (2016).

-• DNA methylation differences between preterm and full-term newborns in genes association with neural function.

33. Weizmann Institute of Science. GeneCards Human Gene Database. www.genecards.org

34. Fontana C, Vitolo MR, Campagnolo PD, Mattevi VS, Genro JP, Almeida S. DRD4 and SLC6A3 gene polymorphisms are associated with food intake and nutritional status in children in early stages of development. J. Nutr. Biochem. 26(12), 1607-1612 (2015).

35. Cansell C, Luquet S. Triglyceride sensing in the reward circuitry: a new insight in feeding behaviour regulation. Biochimie 120, 75-80 (2016).

36. Habak C, Noreau A, Nagano-Saito A et al. Dopamine transporter SLC6A3 genotype affects cortico-striatal activity of set-shifts in Parkinson's disease. Brain 137(Pt 11), 3025-3035 (2014).

37. Yoon DY, Rippel CA, Kobets AJ et al. Dopaminergic polymorphisms in Tourette syndrome: association with the DAT gene (SLC6A3). Am. J. Med. Genet. B Neuropsychiatr. Genet. 144B(5), 605-610 (2007).

38. Huang CC, Lu RB, Shih MC, Yen CH, Huang SY. The dopamine transporter gene possibly affects personality traits in patients with early-onset major depressive disorder. Acta Neuropsychiatr. 25(4), 227-234 (2013).

39. Qadeer MI, Amar A, Mann JJ, Hasnain S. Polymorphisms in dopaminergic system genes; association with criminal behavior and self-reported aggression in violent prison inmates from Pakistan. PLoS ONE 12(6), e0173571 (2017).

40. Ding K, Yang J, Reynolds GP et al. DAT1 methylation is associated with methylphenidate response on oppositional and hyperactive-impulsive symptoms in children and adolescents with ADHD. World J. Biol. Psychiatry 18(4), 291-299 (2016).

41. Bidwell LC, Willcutt EG, McQueen MB et al. A family based association study of DRD4, DAT1, and 5HTT and continuous traits of attention-deficit hyperactivity disorder. Behav. Genet. 41(1), 165-174 (2011).

- Association between SLC6A3 and attention deficit and hyperactivity disorder.

42. Espirito Santo JL, Portuguez MW, Nunes ML. Cognitive and behavioral status of low birth weight preterm children raised in a developing country at preschool age. J. Pediatr. (Rio J.) 85(1), 35-41 (2009).

43. Shumay E, Fowler JS, Volkow ND. Genomic features of the human dopamine transporter gene and its potential epigenetic states: implications for phenotypic diversity. PLoS ONE 5(6), e11067 (2010).

44. Xu Y, Chen XT, Luo M et al. Multiple epigenetic factors predict the attention deficit/hyperactivity disorder among the Chinese Han children. J. Psychiatr. Res. 64, 40-50 (2015).

45. Vucetic Z, Kimmel J, Totoki K, Hollenbeck E, Reyes TM. Maternal high-fat diet alters methylation and gene expression of dopamine and opioid-related genes. Endocrinology 151(10), 4756-4764 (2010).

46. Kitsiou-Tzeli S, Tzetis M. Maternal epigenetics and fetal and neonatal growth. Curr. Opin. Endocrinol. Diabetes Obes. 24(1), 43-46 (2017).

47. Seifuddin F, Wand G, Cox O et al. Genome-wide Methyl-Seq analysis of blood-brain targets of glucocorticoid exposure. Epigenetics 12(8), 637-652 (2017).

48. Arosio B, D'addario C, Gussago C et al. Peripheral blood mononuclear cells as a laboratory to study dementia in the elderly. Biomed. Res. Int. 2014, 169203 (2014). 
49. Montirosso R, Provenzi L, Giorda R et al. SLC6A4 promoter region methylation and socio-emotional stress response in very preterm and full-term infants. Epigenomics 8(7), 895-907 (2016). 
(

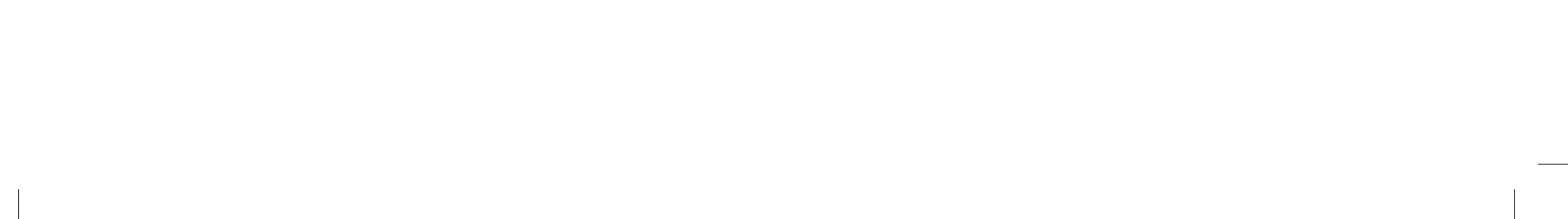




\title{
Evaluating LINE-1 methylation in cleft lip tissues and its association with early pregnancy exposures
}

\author{
Mohammad Faisal J Khan', Julian Little², Peter A Mossey³, Régine PM Steegers-Theunissen, \\ Luca Autelitano5, Ilenia Lombardo', Rita Bassi Andreasi' \& Michele Rubini ${ }^{*, 1}$ \\ 'Department of Biomedical \& Specialty Surgical Sciences, Section of Medical Biochemistry, Molecular Biology \& Genetics, University of Ferrara, \\ Ferrara, Italy \\ ${ }^{2}$ School of Epidemiology and Public Health, University of Ottawa, Ottawa, Ontario, Canada \\ ${ }^{3}$ Craniofacial Development at the WHO-collaborating Centre for Oral \& Craniofacial Research, Dental Hospital \& School, University of Dundee, \\ Dundee, Scotland \\ ${ }^{4}$ Department of Obstetrics \& Gynaecology, Department of Pediatrics, Division Neonatology Erasmus MC, University Medical Center, Rotterdam, \\ The Netherlands \\ ${ }^{5}$ Department of Cranio-Maxillo-Facial Surgery, Regional Centre for Orofacial Clefts \& Craniofacial Anomalies, San Paolo Hospital, University of \\ Milan, Milan, Italy \\ *Author for correspondence: Fax: +39 0532 236157; rub@unife.it
}

\begin{abstract}
Aim: To pilot investigation of methylation of long interspersed nucleotide element-1 in lip tissues from infants with nonsyndromic cleft lip, and its association with maternal periconceptional exposures. Methods: The lateral and medial sides of the cleft lips of 23 affected infants were analyzed for long interspersed nucleotide element-1 methylation by bisulfite conversion and pyrosequencing. Results: The medial side showed $1.8 \%$ higher methylation compared with the lateral side; $p=0.031$, particularly in male infants $(2.7 \%$ difference; $p=0.011)$ or when the mothers did not take folic acid during periconceptional period $(2.4 \%$ difference; $p=0.011)$. These results were not statistically significant when Bonferroni adjustment was used. Conclusion: The observed differences in DNA methylation, although nonsignificant after correction for multiple comparisons, suggest that differential regulation of the two sides may impact lip fusion and warrant larger-scale replication.
\end{abstract}

First draft submitted: 28 June 2017; Accepted for publication: 22 September 2017; Published online: 29 November 2017

Keywords: cleft lip with or without cleft palate $\bullet$ DNA methylation $\bullet$ LINE-1

Orofacial clefts (OFC) are congenital anomalies affecting the lip, palate or both and categorized in two broad phenotypes, cleft lip with or without cleft palate $(\mathrm{CL} / \mathrm{P})$ and cleft palate $(\mathrm{CP})[1]$. The occurrence of the two phenotypes shows a sex-based disparity, with a male predominance in CL/P and a female predominance in $\mathrm{CP}[2,3]$. About $30 \%$ of cases of OFC are syndromic. The nonsyndromic cases are thought to be due to multiple genes and environmental factors [4,5].

Like most other chronic diseases, the heritability of developing nonsyndromic OFC is only to a small extent accounted for by the major risk loci so far identified, indicating that the infant's genetic profile alone cannot explain the origin of this malformation. And as the formation of the lip and palate starts early in pregnancy, is vulnerable to perturbation of the maternal nutritional and nonnutritional milieu [6], which can affect its epigenetic programming. Therefore, cleft of the lip and palate can arise as a result of any change that impacts its normal development such as genetic variation $[7,8]$, and environmental factors including maternal nutrients, smoking and hormones $[9,10,11,12]$, but the role of these factors in etiology is still inconclusive $[13,14]$.

Numerous studies suggest that the risk for OFC is increased by disturbance of the one-carbon metabolism cycle [15], although the role of specific nutrients such as folate remains controversial [16,17,18]. Folate feeds into the one-carbon metabolism cycle that results in the formation of methyl groups [19]. The level of supplementation of methyl donors in pregnancy has been shown to influence the levels of DNA methylation in infants [20], espe- 
cially in the periconceptional period [21]. Moreover, in a mouse study, an increase in the level of dietary methyl donors has been found to increase genomic DNA methylation levels in the offspring [22].

DNA methylation of the pyrimidine base cytosine in DNA may be one of the mechanisms underlying differential programming of cell lineages in mammalian development, as suggested by the erasing or reshuffling of methylation marks in the early embryo and its re-establishment after implantation $[23,24,25]$. This process establishes basic adult methylation patterns prior to organogenesis. DNA methylation is in a state of flux during gametogenesis and early embryogenesis, which can be modulated by embryonic environmental exposures. Potentially, investigation of the methylation of long interspersed nucleotide element-1 (LINE-1) repetitive elements, generally accepted to be a surrogate measure of global DNA methylation content [26,27], could provide insight into the role of the environment in regulating whole-genome DNA methylation.

Numerous studies have shown changes in LINE-1 DNA methylation associated with the onset of specific conditions such as gestational diabetes, preeclempsia, congenital heart diseases ( $\mathrm{CHDs}$ ) and neural tube defects (NTDs) [28,29,30,31] and with prognosis of diseases such as several types of cancer [32,33,34]. Studies in animals or animal-derived tissues have shown the involvement of DNA methylation in the development of OFC, one study involved lip tissue [35], but the majority of investigations are limited to secondary palate tissues with an intact upper lip [36,37]. Most human OFC cases involve clefting of the upper lip [38,39,40]. Moreover, the lip and palate have separate embryological origins and therefore may have different etiologies and DNA methylation status [41]. This is supported by recent data showing distinct methylation profile in different cleft subtypes using blood DNA [42]. In addition, Alvizi et al. observed that DNA methylation correlated with the penetrance of nonsyndromic cleft lip and palate (nsCL/P) [43].

There is a lack of epigenetic data on the DNA methylation of cleft tissues in humans. To overcome this shortcoming, we undertook a pilot study of LINE-1 methylation in lip tissue taken from humans undergoing surgical repair of cleft lip (CL) to address epigenetic changes. We also investigated the association of LINE-1 methylation with reported maternal periconceptional folic acid (FA) supplementation, sex and cleft subtype.

\section{Materials \& methods}

Cases

Infants with nonsyndromic $\mathrm{CL}$ with or without palate were identified in the context of the ongoing PENTACLEFT project [44]. The PENTACLEFT project protocol includes the recruitment of nonsyndromic CL/P cases, their parents and maternal grandparents, and the collection of genomic DNA from peripheral blood or buccal swab samples. The project was approved by local institutional review board (prot. no. 08-2011), and case enrollment required written parental informed consent. Families of consecutive cases were invited to enroll in the study at the Regional Centre for Orofacial Clefts and Craniofacial Anomalies, San Paolo Hospital, Milan, Italy, at the time of the first surgical intervention on the index child. Infants with recognized syndromic clefts or the Pierre Robin sequence were excluded from the study. Parents of included infants were asked to respond to a specific questionnaire that was administered by personal interview when the affected child was brought to the surgical center to undergo the primary surgery. Information on educational status, ethnic group and family history of both parents was collected, along with data on maternal medical and reproductive history, exposure to environmental risk factors, use of drugs, medications and supplements such as FA during the periconceptional period (from 3 months before to 3 months after conception). Lip tissue samples were collected from nonsyndromic $\mathrm{CL} / \mathrm{P}$ cases at the time of first surgery.

\section{Tissue samples}

The lip tissue samples were collected from twenty-three nonsyndromic CL/P cases, with an average age of 6.5 (95\% CI: 5.0-7.1) months at the time of surgery: 12 males (seven CL, five cleft lip and palate [CLP]; three cases with preconceptional FA [preFA] and nine without preconceptional FA [No-preFA]); and 11 females (six CL, five CLP; two cases with preFA, seven No-preFA and two with missing preFA data). Samples were collected immediately in lysis buffer ( $\mathrm{pH} 7.4$ ) from both lateral and medial side of CL. The samples were then transferred to the laboratory at University of Ferrara where they were processed for epigenetic study.

\section{DNA extraction \& sodium bisulfite treatment}

The cases' lateral and medial side CL tissues collected in lysis buffer were homogenized separately, with a view to primarily include the connective tissue portion of the upper lip, with minimum contribution from the epidermis. The genomic DNA was extracted from the homogenate using Nucleon BACC1 kit (Amersham Biosciences, 
part of GE Healthcare Europe, Switzerland) according to the manufacturer's instructions and quantified using Qubit $^{\circledR}$ dsDNA BR Assay Kit (Life technologies, OR, USA). The DNA with concentration $>10$ ng on Qubit ${ }^{\circledR}$ instrument was selected and bisulfite converted using EZ-DNA Methylation-Lightning ${ }^{\text {TM }}$ Kit (Zymo Research, CA, USA).

\section{Pyrosequencing}

The LINE-1 DNA methylation level was measured for all the study samples with pyrosequencing on PyroMarkQ96 ID using PyroMark Gold reagents (Qiagen GmbH, Hilden, Germany). LINE-1 region including 4 CpG sites (position 305-331 in accession no. X58075) was amplified by PCR using the following primers: 5'-TTTTGAGTTAGGTGTGGGATATA-3' and 5'-Bio-AAATCAAAAAATTCCCTTTC-3'. LINE-1 PCR products represent a pool of approximately 15,000 genomic loci interspersed across the whole human genome $[45,46]$. PCR reactions were performed in duplicate to achieve precision between runs with total volume of $25 \mu \mathrm{l}$ containing $10 \times$ PCR buffer, $50 \mathrm{mM} \mathrm{MgCl}_{2}, 2.5 \mathrm{mM}$ deoxynucleotide triphosphates (dNTPs), $10 \mathrm{pM}$ of each primer, $5 \mathrm{U}$ Taq polymerase and $2.5 \mu \mathrm{l}$ of bisulfite-modified DNA with the following cycling profile: 27 cycles of $94^{\circ} \mathrm{C}$ for $15 \mathrm{~s}, 60^{\circ} \mathrm{C}$ for $30 \mathrm{~s}$ and $72^{\circ} \mathrm{C}$ for $30 \mathrm{~s}$, followed by $72^{\circ} \mathrm{C}$ for $2 \mathrm{~min}$. The amplicon of $147 \mathrm{bp}$ was analyzed on $8 \%$ polyacrylamide gel using silver staining. The PCR plate with each well contained $20 \mu \mathrm{l}$ of PCR product, $20 \mu \mathrm{l}$ of RNAse-free distilled water, $3 \mu \mathrm{l}$ of sepharose beads containing streptavidin and $37 \mu \mathrm{l}$ of binding buffer; thus a total volume of $80 \mu \mathrm{l}$ of the mixture was placed on the thermo-mixer. Following this, the PCR product was made single stranded to act as a template in a pyrosequencing reaction by washing with ethanol $70 \%$ and denaturation buffer using a Pyrosequencing Vacuum Prep Tool (Biotage AB, Uppsala, Sweden).

The pyrosequencing runs were performed to obtain a pyrogram from each PCR reaction, using software for analysis in allele-quantification mode, in a total volume of $40 \mu \mathrm{l}$ per well, including $38.4 \mu \mathrm{l}$ of annealing buffer and $1.6 \mu \mathrm{l}$ of $10 \mathrm{pM}$ sequencing primer with suspended beads containing the sample DNA. The assays were created according to the manufacturer's recommendations and the output of the two pyrosequencing runs was averaged. The nucleotide dispensation order was: ACTCAGTGTGTCAGTCAGTTAGTCTG. LINE-1 DNA methylation values were detected at positions $+306,+318,+321$ and +328 in Genebank sequence X58075. The CpG site at position +328 was not considered for subsequent analyses, as precision of methylation values was insufficient, probably due to the adjacent CT dinucleotide. Using the combined average data, the overall LINE-1 DNA methylation values was calculated as the mean of the proportions of $\mathrm{C}(\%)$ at the $3 \mathrm{CpG}$ sites analyzed, (positions $+306,+318$ and +321 ) and this indicated the level of methylation of LINE-1 elements [47].

\section{Statistical analysis}

All the statistical analysis was performed using the IBM SPSS Statistics 21 (IBM Corp., NY, USA). All p-values were two sided, with a threshold for declaring statistical significance of $\mathrm{p}<0.05$. The distributions of LINE-1 methylation levels were checked for normality using the Shapiro-Wilk test that is appropriate for small samples; none departed from normality. For within case comparison between lateral and medial cleft side, a paired student's $t$-test was used. For comparison of two-level categories of periconceptional use of supplements containing FA, sex and cleft subtype, the unpaired student's $t$-test was performed. In view of possible concerns about multiple comparisons, we also applied the Bonferroni correction to comparisons within and between cases. This was a secondary analysis because of the known limitations of the Bonferroni correction and inapplicability of other forms of adjustment to this study [48,49]. We adopted the most conservative approach of adjusting for all 13 comparisons reported.

\section{Results}

Our results are based on samples that showed a normal distribution of LINE-1 methylation for both medial $(\mathrm{p}=0.124)$ and lateral $(\mathrm{p}=0.773)$ sides. Initial analysis using the nominal $\mathrm{p}<0.05$ threshold showed that the DNA in tissue taken from the medial side of the CL was found to have $1.8 \%$ more methylation compared with DNA in tissue taken from the lateral side ( $p=0.031$; Table 1). In analysis stratified on sex, no significant difference in methylation between the sexes for either the lateral (males $71.5 \pm 3.1 \%$ verses females $72.1 \pm 1.2 \%$; $\mathrm{p}=0.748$ ) or medial (males $74.2 \pm 3.0 \%$ verses females $73.0 \pm 2.4 \% ; \mathrm{p}=0.293$ ) sides was observed (Figure 1). However, the methylation levels between lateral and medial sides in males were observed to be significantly different (lateral $71.5 \pm 3.10 \%$ verses medial $74.2 \pm 3.0 \% ; \mathrm{p}=0.011$ ) (Table 1). When methylation levels within and between CL and CLP subtype were analyzed, no significant differences were observed (Table 1). To evaluate the role of periconceptional FA as an environmental factor affecting the level of global DNA methylation, we calcu- 


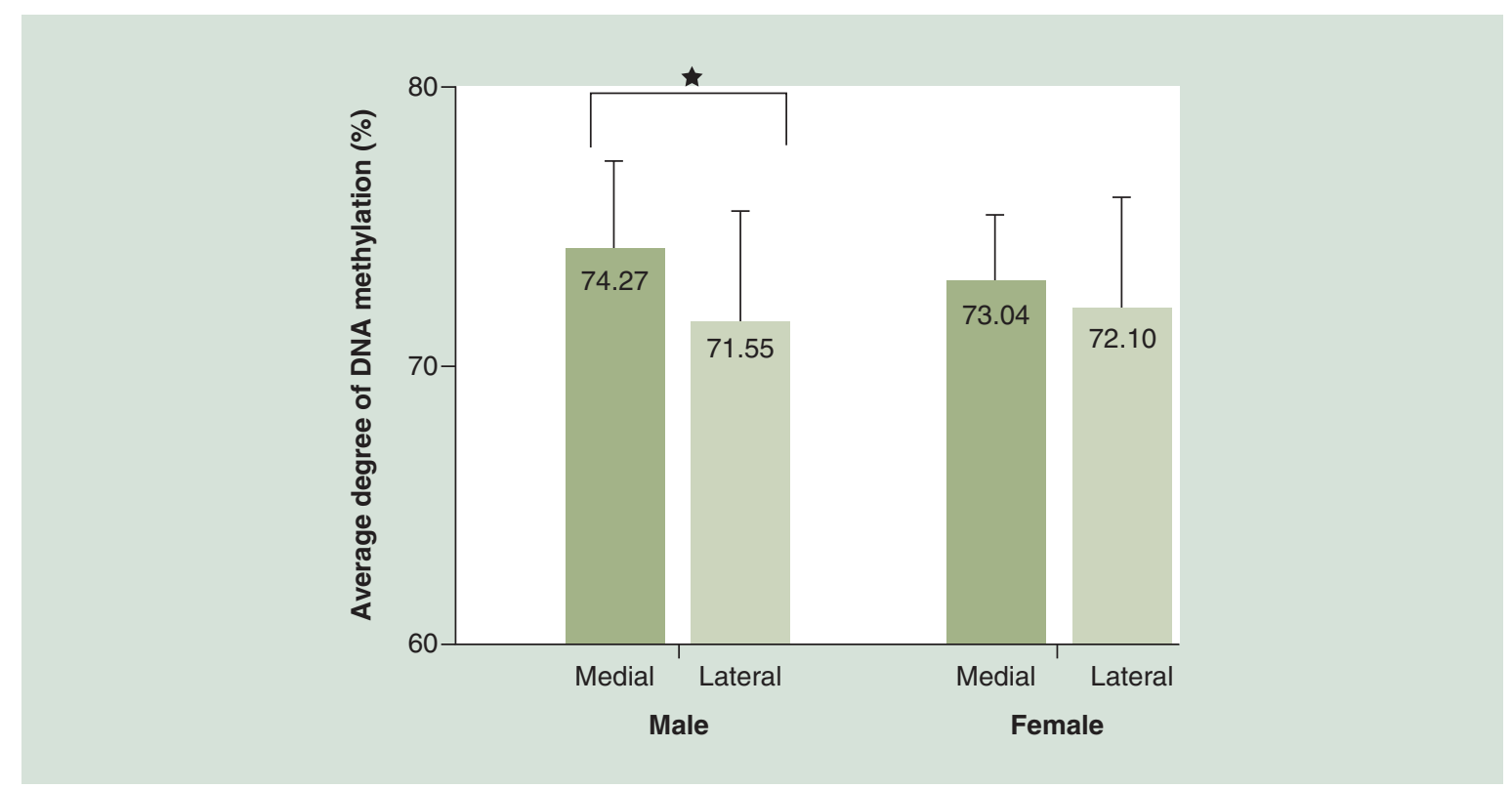

Figure 1. Average degree of long interspersed nucleotide element-1 DNA methylation on medial and lateral cleft side with sex. Bars represent standard deviation.

lated the average methylation level on lateral and medial cleft sides in infants born to mothers with and without periconceptional FA supplementation. Comparison between these two groups showed no significant difference. However, methylation on the medial side was $2.7 \%$ higher than that on the lateral side in the cases whose mothers did not take periconceptional FA supplements ( $p=0.011$; Table 1$)$. In secondary analysis using a Bonferronicorrected threshold $(\mathrm{p}=0.004)$, this finding was not statistically significant.

\section{Discussion}

In this first pilot study using human lip tissue obtained from infants with nonsyndromic CL/P, we observed differences in LINE-1 DNA methylation between tissues on the lateral and medial side of the cleft. These differences were apparent in boys but not in girls, and in infants whose mothers did not take supplements containing FA in the periconceptional period but not in the offspring of women who took supplements. There were no differences in methylation by sex or cleft subgroup.

From our results it appears that the medial sides of clefts have higher global methylation levels, especially in male infants. This pattern is also apparent in infants from pregnancies in which supplements containing FA were not taken during the periconceptional period, but we acknowledge that numbers are very small. We also recognize that the inability to obtain normal lip tissues with which to compare our lip tissue samples is a shortcoming that prevents the direct determination of whether this epigenetic difference between the two sides is a pattern present in the general population, rather than being specific to clefting, that is, a real cause or consequence of clefting. However, we suggest that this difference may reflect the fact that these tissues develop during separate embryonic stages and therefore possibly experience different environmental exposures. The lateral aspects of the upper lip originate from the maxillary process during the 4th week of embryonic development, while the medial aspects of the upper lip originate from the medial nasal process, beginning in the 5th week [4]. It is possible that the two separate windows of origin may have been exposed to different environmental milieus that resulted in differential methylation of the two sides of the cleft, in turn influencing the closure of the two processes and the occurrence of a CL.

Another possible explanation for the observed differences in methylation of the two cleft sides could lie in the developmental field concept, and act in a spatial and temporal manner [50]. For example, in normal circumstances, the lateral and medial aspects of the upper lip originate from two different embryonic units that constitute a morphogenetic reactive unit. It is possible that this part of the embryo's reactive unit in the two aspects of the upper lip may have experienced different spatial and temporal forces of organization and differentiation (epimorphic field) leading to dysmorphogenesis of the two sides, reflected as a difference in methylation as observed in our study. Some empirical support is provided by a recent animal study that shows temporal regulation of 
Sonic Hedgehog, resulting in downregulation of Foxf2 expression and reduced proliferation of medial nasal process mesenchymal cells that are required for upper lip closure [51]. We postulate that differential expression of a single gene on the two sides of the developing lip could be regulated by different epistatic factors, and hence we plan to investigate expression of specific genes implicated in human clefting in future work.

An influence of in utero environment on epigenetic modulation is compatible with previous reports showing associations between DNA methylation changes and neural tube defects [52] and congenital heart defects [53,54]. Neural tube defects are clearly linked with low folate status [55], and there is some evidence that this is also the case for several types of congenital heart defects [56]. There appears to be no previous reports on the association between FA intake during the periconceptional period or pregnancy and global LINE-1 DNA methylation in humans in general, or specific to the development of CL/P [57]. In infants born to mothers who did not use periconceptional FA supplementation we found a suggestion of a trend of increased methylation on the medial side.

In the cleft subgroup analysis, we found no significant difference in methylation between the two sides, a result that is compatible with the similar DNA methylation profile of CL and CLP reported by Sharp et al. [42]. Similarly, sex subgroup analysis showed no significant difference in methylation between the two sides. However, comparison within males showed a significantly higher methylation for the medial side that may be an outcome of differential developmental programming in males, who have an increased susceptibility to CL/P $[2,3]$. This may reflect a role of sex in cleft etiology. We acknowledge that our results are based on small numbers, because collecting tissues from the cleft cases is of great challenge [58]. Statistical power is low, and we note that all nominally significant results in this study were nonsignificant in the secondary analysis applying the Bonferroni correction. Therefore, we urge that until replication of our results with in a larger sample size, the clear answer to this primarily evidence should be taken with caution.

According to a recent study, infants with CL and CLP subtypes may have similar rates of development, suggesting that epigenetic changes associated with development may not be a confounding factor in epigenetic stud-

Table 1. Global long interspersed nucleotide element-1 DNA methylation level (\%) of medial and lateral cleft side.

\begin{tabular}{|c|c|c|c|}
\hline & Medial side \pm SD & Lateral side \pm SD & $\begin{array}{l}\text { Mean difference }(95 \% \\
\mathrm{CI}), \mathrm{p} \text {-value }\end{array}$ \\
\hline Total cases $(n=23)^{\dagger}$ & $73.68 \pm 2.75$ & $71.81 \pm 3.89$ & $\begin{array}{l}1.87(0.18-3.55) \\
p=0.031\end{array}$ \\
\hline Males $(n=12)$ & $74.27 \pm 3.03$ & $71.55 \pm 3.10$ & $\begin{array}{l}2.71(0.74-4.69) \\
p=0.011\end{array}$ \\
\hline Females $(n=11)$ & $73.04 \pm 2.40$ & $72.10 \pm 1.20$ & $\begin{array}{l}0.94(-2.13-4.01) \\
p=0.510\end{array}$ \\
\hline $\begin{array}{l}\text { Mean difference } \\
(95 \% \mathrm{Cl}) \mathrm{p} \text {-value }\end{array}$ & $\begin{array}{l}1.23(-1.14-3.61) \\
p=0.293\end{array}$ & $\begin{array}{l}-0.54(-3.10-2.91) \\
\mathrm{p}=0.748\end{array}$ & \\
\hline$C L(n=13)$ & $74.01 \pm 2.56$ & $72.13 \pm 3.36$ & $\begin{array}{l}1.87(-0.38-4.13) \\
p=0.095\end{array}$ \\
\hline $\operatorname{CLP}(n=10)$ & $73.26 \pm 3.07$ & $71.40 \pm 4.43$ & $\begin{array}{l}1.86(-1.20-4.93) \\
p=0.204\end{array}$ \\
\hline $\begin{array}{l}\text { Mean difference } \\
(95 \% \mathrm{Cl}) \mathrm{p} \text {-value }\end{array}$ & $\begin{array}{l}0.74(-1.70-3.20) \\
p=0.525\end{array}$ & $\begin{array}{l}0.73(-2.73-4.20) \\
p=0.252\end{array}$ & \\
\hline No-preFA $(n=16)$ & $73.78 \pm 2.91$ & $71.32 \pm 4.03$ & $\begin{array}{l}2.46(0.64-4.27) \\
p=0.011\end{array}$ \\
\hline preFA $(n=5)$ & $72.77 \pm 2.72$ & $72.40 \pm 2.58$ & $\begin{array}{l}0.37(-6.07-6.81) \\
p=0.880\end{array}$ \\
\hline $\begin{array}{l}\text { Mean difference } \\
(95 \% \mathrm{Cl}) \mathrm{p} \text {-value }\end{array}$ & $\begin{array}{l}1.01(-2.07-4.09) \\
p=0.594\end{array}$ & $\begin{array}{l}-1.08(-5.12-2.97) \\
p=0.442\end{array}$ & \\
\hline \multicolumn{4}{|c|}{$\begin{array}{l}\text { Mean } \pm \text { SD values of total CL/P cases or cases categorized by sex, cleft subtype and preFA supplementation are shown, along with mean } \\
\text { difference, } 95 \% \text { Cl and nominal p-value of } t \text {-test. } \\
\text { 'Information on preFA use was missing for two cases, both of whom were female. } \\
\text { CL: Cleft lip; CL/P: Cleft lip with or without cleft palate; CLP: Cleft lip and palate; No-preFA: No periconceptional folic acid; } \\
\text { preFA: Periconceptional folic acid; SD: Standard deviation. }\end{array}$} \\
\hline
\end{tabular}


ies of CL and CLP [42]. Children with CL and CLP underwent surgery and thus had samples taken at approximately similar ages. There are reports of the absence of an age effect on LINE-1 methylation [59,60,61], and from a study on lip tissues collected from 4-month-old CL and CLP cases that show an independent expression of genes associated with aging [60]. Therefore, we consider that our observation is little influenced by aging. Moreover, the advantage of using tissues derived from same individuals with relatively similar age in our study overcomes the influence of DNA sequence on DNA methylation and possibly the influence of age on DNA methylation, if any. Although the targeting of lip tissues in this study potentially would give the most direct insight into epigenetic changes associated with the occurrence of $\mathrm{CL}$, we are aware that heterogeneity could arise in these tissues from neural-crest-derived connective tissue and muscles, and in situ-derived epidermis. However, we sought to overcome this limitation by collecting tissues in a lysis buffer to minimize the contribution from the epidermis and primarily include the connective tissue portion of lip tissues. Moreover, the observation of similar correlations between blood and tissue methylation in nsCL/P epigenetic study of Sharp et al. [42] and Alvizi et al. [43] suggests that the two tissues can be considered to be exchangeable in nsCL/P methylation studies at least. An aim of our future work in newly recruited cases is to collect blood and investigate correlation between methylation in blood with that in tissue from the lateral and medial sides of clefts.

Another potential limitation of our study is that the tissue DNA methylation measurement in infancy may have been indirectly influenced by the presence of a CL and so may differ from that at the time of lip fusion in embryonic development. But for ethical reasons, this is the only accessible and the closest tissue associated with OFC that can be studied in humans. It is obviously difficult to collect lip-tissue specimens from normal babies and this limits making direct causal inference.

Of note, our study is based on small sample size (with possibility of both type I and type II errors), and being aware of this limitation, splitting our samples based on factors (sex, cleft subtype and pre-FA) thought to affect methylation, was an attempt to provide preliminary data. Our primary analysis did not include Bonferroni correction because of known limitations including type II error [48] and the inapplicability of other forms of adjustment [49] in this exploratory study, in view of being cautious of not missing a possible effect worthy of future investigation. Since the recruitment of cleft cases is still ongoing in the PENTACLEFT project, we hope to replicate and better justify our preliminary finding using larger number of cases and to investigate epistatic regulation of genes implicated in OFC.

\section{Conclusion}

The observed difference in methylation between tissue taken from the lateral and medial sides of a CL may reflect the fact that these tissues develop during separate embryonic stages and therefore possibly experience different environmental exposures that can regulate DNA methylation patterns differently. The finding of a difference in DNA methylation in male but not female infants should be further investigated. Our findings suggest that epigenetic mechanisms may be important in the etiology of OFC, warranting replication in a larger study.

Summary points

- The etiology of nonsyndromic orofacial cleft (OFC) is only in part explained by genetic variants. We hypothesized the possible role of early pregnancy epigenetic programming in the pathogenesis of orofacial cleft.

- There is lack in epigenetic data on the DNA methylation of cleft tissues in humans. Therefore, to overcome this shortcoming, this pilot study is the first comparative assessment of long interspersed nucleotide element-1 methylation between tissues taken from the two sides of infants with cleft lip, and investigate possible association with reported maternal periconceptional environmental exposures.

- We show that long interspersed nucleotide element-1 methylation of tissues from medial side of the lip is higher compared with the lateral side, and that is particularly apparent for male infants. In addition, we show that the medial side methylation is higher for infants whose mothers did not take supplements containing folic acid during periconceptional period.

- The observed differences in methylation between tissue taken from lateral and medial sides of cleft lip may reflect the fact that these tissues develop during separate embryonic stages and therefore possibly experience different environmental exposures that can modulate DNA methylation patterns differently.

- The differences in methylation between males and females may reflect a play of chance.

- This study suggests differential methylation of two cleft side that may impact lip fusion, warranting replication in a larger study. 


\section{Financial \& competing interests disclosure}

The authors have no relevant affiliations or financial involvement with any organization or entity with a financial interest in or financial conflict with the subject matter or materials discussed in the manuscript. This includes employment, consultancies, honoraria, stock ownership or options, expert testimony, grants or patents received or pending, or royalties.

No writing assistance was utilized in the production of this manuscript.

\section{Ethical conduct of research}

Research ethical approval was granted by the local IRB (prot. N.08-2011) and required written parental informed consent was collected for enrolled case.

\section{References}

Papers of special note have been highlighted as: • of interest; $\bullet \bullet$ of $\quad$ considerable interest

1 Marazita ML. The evolution of human genetic studies of cleft lip and cleft palate. Annu. Rev. Genomics Hum. Genet. 13, 263-283 (2012).

2 Fogh-Andersen P. Incidence of cleft lip and palate: constant or increasing? Acta. Chir. Scand. 122, 106-111 (1961).

3 Mossey PA, Little J. Epidemiology of oral clefts: an international perspective. In: Cleft Lip And Palate: From Origin To Treatment. Wyszynski DF (Ed.). Oxford University Press, NY, USA, 127-158 (2002).

4 Dixon MJ, Marazita ML, Beaty TH, Murray JC. Cleft lip and palate: understanding genetic and environmental influences. Nat. Rev. Genet. 12(3), 167-178 (2011).

- Describes the embryonic development of lip and palate with potential role of epigenetics in the etiology of orofacial cleft.

5 Mangold E, Ludwig KU, Nöthen MM. Breakthroughs in the genetics of orofacial clefting. Trends Mol. Med. 17(12), 725-733 (2011).

6 Desai M, Jellyman JK, Ross MG. Epigenomics, gestational programming and risk of metabolic syndrome. Int. J. Obes. (Lond) 39(4), 633-641 (2015)

- Describes the role of nutrients in early gestational programming that can affect the epigenome.

7 Dubois L, Ohm Kyvik K, Girard M et al. Genetic and environmental contributions to weight, height, and BMI from birth to 19 years of age: an international study of over 12,000 twin pairs. PLoS ONE 7(2), e30153 (2012).

8 Godfrey KM, Barker DJ. Fetal programming and adult health. Public Health Nutr. 4(2B), 611-624 (2001).

9 Grieger JA, Clifton VL. A review of the impact of dietary intakes in human pregnancy on infant birthweight. Nutrients $7(1)$, 153-178 (2014).

10 Walsh JM, Mcauliffe FM. Impact of maternal nutrition on pregnancy outcome - does it matter what pregnant women eat? Best Pract Res Clin Obstet Gynaecol 29(1), 63-78 (2015).

11 Martelli DR, Coletta RD, Oliveira EA et al. Association between maternal smoking, gender, and cleft lip and palate. Braz. J. Otorhinolaryngol. 81(5), 514-519 (2015).

12 Gresham E, Byles JE, Bisquera A, Hure AJ. Effects of dietary interventions on neonatal and infant outcomes: a systematic review and meta-analysis. Am. J. Clin. Nutr. 100(5), 1298-1321 (2014).

13 Murray JC. Gene/environment causes of cleft lip and/or palate. Clin. Genet. 61(4), 248-256 (2002).

14 Yuan Q, Blanton SH, Hecht JT. Genetic causes of nonsyndromic cleft lip with or without cleft palate. Adv. Otorhinolaryngol. 70, 107-113 (2011).

15 Ebadifar A, Ameli N, Khorramkhorshid HR, Salehi Zeinabadi M, Kamali K, Khoshbakht T. Incidence assessment of MTHFR C677T and A1298C polymorphisms in Iranian non-syndromic cleft lip and/or palate patients. J. Dent. Res. Dent. Clin. Dent. Prospects 9(2), 101-104 (2015).

16 Johnson CY, Little J. Folate intake, markers of folate status and oral clefts: is the evidence converging? Int. J. Epidemiol. 37(5), 1041-1058 (2008).

17 Butali A, Little J, Chevrier C et al. Folic acid supplementation use and the MTHFR C677T polymorphism in orofacial clefts etiology: an individual participant data pooled-analysis. Birth Defects Res. A. Clin. Mol. Teratol. 97(8), 509-514 (2013).

18 Rozendaal AM, Van Essen AJ, Te Meerman GJ et al. Periconceptional folic acid associated with an increased risk of oral clefts relative to non-folate related malformations in the Northern Netherlands: a population based case-control study. Eur. J. Epidemiol. 28(11), 875-887 (2013).

19 Fox JT, Stover PJ. Folate-mediated one-carbon metabolism. Vitamins and hormones 79, 1-44 (2008).

20 Sinclair KD, Allegrucci C, Singh R et al. DNA methylation, insulin resistance, and blood pressure in offspring determined by maternal periconceptional B vitamin and methionine status. Proc. Natl Acad. Sci. USA 104(49), 19351-19356 (2007). 
21 Pauwels S, Ghosh M, Duca RC et al. Maternal intake of methyl-group donors affects DNA methylation of metabolic genes in infants. Clin. Epigenet. 9, 16 (2017).

- Shows the role of periconceptional methyl donors in modulating DNA methylation.

22 Hollingsworth JW, Maruoka S, Boon K et al. In utero supplementation with methyl donors enhances allergic airway disease in mice. J. Clin. Invest. 118(10), 3462-3469 (2008).

23 Miyoshi N, Stel JM, Shioda K et al. Erasure of DNA methylation, genomic imprints, and epimutations in a primordial germ-cell model derived from mouse pluripotent stem cells. Proc. Natl Acad. Sci. USA 113(34), 9545-9550 (2016). Kafri T, Ariel M, Brandeis M et al. Developmental pattern of gene-specific DNA methylation in the mouse embryo and germ line. Genes Dev. 6(5), 705-714 (1992).

25 Reik W, Santos F, Mitsuya K, Morgan H, Dean W. Epigenetic asymmetry in the mammalian zygote and early embryo: relationship to lineage commitment? Philos. Trans. R. Soc. Lond. B Biol. Sci. 358(1436), 1403-1409; discussion 1409 (2003).

26 Fryer AA, Nafee TM, Ismail KM, Carroll WD, Emes RD, Farrell WE. LINE-1 DNA methylation is inversely correlated with cord plasma homocysteine in man: a preliminary study. Epigenetics 4(6), 394-398 (2009).

27 Yang AS, Estecio MR, Doshi K, Kondo Y, Tajara EH, Issa JP. A simple method for estimating global DNA methylation using bisulfite PCR of repetitive DNA elements. Nucleic Acids Res. 32(3), e38 (2004).

28 Reichetzeder C, Dwi Putra SE, Pfab T et al. Increased global placental DNA methylation levels are associated with gestational diabetes. Clin. Epigenetics 8, 82 (2016).

29 Choudhury M, Friedman JE. Epigenetics and microRNAs in preeclampsia. Clin. Exp. Hypertens. 34(5), 334-341 (2012).

30 Stolk L, Bouwland-Both MI, Van Mil NH et al. Epigenetic profiles in children with a neural tube defect; a case-control study in two populations. PLoS ONE 8(11), e78462 (2013).

31 Wang L, Wang F, Guan J et al. Relation between hypomethylation of long interspersed nucleotide elements and risk of neural tube defects. Am. J. Clin. Nutr. 91(5), 1359-1367 (2010).

32 Furlan C, Polesel J, Barzan L et al. Prognostic significance of LINE-1 hypomethylation in oropharyngeal squamous cell carcinoma. Clin. Epigenet. 9 , 58 (2017).

33 Ye D, Jiang D, Li Y, Jin M, Chen K. The role of LINE-1 methylation in predicting survival among colorectal cancer patients: a meta-analysis. Int. J. Clin. Oncol. 22(4), 749-757 (2017).

34 Ogino S, Nosho K, Kirkner GJ et al. A cohort study of tumoral LINE-1 hypomethylation and prognosis in colon cancer. J. Natl Cancer Inst. 100 (23), 1734-1738 (2008).

35 Juriloff DM, Harris MJ, Mager DL, Gagnier L. Epigenetic mechanism causes Wnt9b deficiency and nonsyndromic cleft lip and palate in the A/WySn mouse strain. Birth Defects Res. A. Clin. Mol. Teratol. 100(10), 772-788 (2014).

36 Mukhopadhyay P, Greene RM, Pisano MM. Cigarette smoke induces proteasomal-mediated degradation of DNA methyltransferases and methyl CpG-/CpG domain-binding proteins in embryonic orofacial cells. Reprod. Toxicol. 58, 140-148 (2015).

- Describes the potential role of environment in modulating DNA methylation of early embryonic tissues that contribute to the formation of lip.

37 Seelan RS, Mukhopadhyay P, Warner DR, Webb CL, Pisano M, Greene RM. Epigenetic regulation of Sox4 during palate development. Epigenomics 5(2), 131-146 (2013).

38 Watkins SE, Meyer RE, Strauss RP, Aylsworth AS. Classification, epidemiology, and genetics of orofacial clefts. Clin. Plast. Surg. 41(2), 149-163 (2014).

39 Gritli-Linde A. The etiopathogenesis of cleft lip and cleft palate: usefulness and caveats of mouse models. Curr. Top. Dev. Biol. 84, 37-138 (2008).

40 Gritli-Linde A. The mouse as a developmental model for cleft lip and palate research. Front. Oral Biol. 16, 32-51 (2012).

41 Pansky B. Review Of Medical Embryology. Embryome Sciences, Inc 1301 Harbor Bay Parkway, CA, USA, 94502 (1982).

42 Sharp GC, Ho K, Davies A et al. Distinct DNA methylation profiles in subtypes of orofacial cleft. Clin. Epigenetics 9, 63 (2017).

-• Benchmarking study showing methylation in human cleft subtypes.

43 Alvizi L, Ke X, Brito LA et al. Differential methylation is associated with non-syndromic cleft lip and palate and contributes to penetrance effects. Sci. Rep. 7(1), 2441 (2017).

- Benchmarking study showing contribution of methylation in penetrance effects in human nonsyndromic cleft lip and palate.

44 University of Ferrara, projects. www.unife.it/progetto/pentacleft.

45 Knothe C, Shiratori H, Resch E et al. Disagreement between two common biomarkers of global DNA methylation. Clin. Epigenet. 8, 60 (2016).

46 Yang AS, Estecio MR, Doshi K, Kondo Y, Tajara EH, Issa JP. A simple method for estimating global DNA methylation using bisulfite PCR of repetitive DNA elements. Nucleic Acids Res. 32(3), e38 (2004). 
Tournier B, Chapusot C, Courcet E et al. Why do results conflict regarding the prognostic value of the methylation status in colon cancers? The role of the preservation method. BMC Cancer 12, 12 (2012).

Opitz JM. The developmental field concept in clinical genetics. J. Pediatr. 101(5), 805-809 (1982).

- Describes the concept of epimorphic field in an embryo that reacts in a spatially and temporally coordinated manner to the localized forces of organization and differentiation.

49 Perneger TV. What's wrong with Bonferroni adjustments. BMJ 316(7139), 1236-1238 (1998).

50 Armstrong RA. When to use the Bonferroni correction. Ophthalmic \& physiological optics: the journal of the British College of Ophthalmic. Opticians 34(5), 502-508 (2014).

51 Everson JL, Fink DM, Yoon JW et al. Sonic Hedgehog regulation of Foxf2 promotes cranial neural crest mesenchyme proliferation and is disrupted in cleft lip morphogenesis. Development 144(11), 2082-2091 (2017).

-. Benchmarking study showing temporal regulation of gene in the medial aspect of a cleft lip.

52 Wang X, Guan Z, Chen Y et al. Genomic DNA hypomethylation is associated with neural tube defects induced by methotrexate inhibition of folate metabolism. PLoS ONE 10(3), e0121869 (2015).

53 Serra-Juhe C, Cusco I, Homs A, Flores R, Toran N, Perez-Jurado LA. DNA methylation abnormalities in congenital heart disease. Epigenetics 10(2), 167-177 (2015).

54 Sheng W, Qian Y, Zhang P et al. Association of promoter methylation statuses of congenital heart defect candidate genes with Tetralogy of Fallot. J. Transl. Med. 12, 31 (2014).

55 Arth A, Kancherla V, Pachon H, Zimmerman S, Johnson Q, Oakley GP Jr. A 2015 global update on folic acid-preventable spina bifida and anencephaly. Birth Defects Res. A. Clin. Mol. Teratol. 106(7), 520-529 (2016).

56 Liu S, Joseph KS, Luo W et al. Effect of folic acid food fortification in Canada on congenital heart disease subtypes. Circulation 134(9), 647-655 (2016).

57 Geraghty AA, Lindsay KL, Alberdi G, McAuliffe FM, Gibney ER. Nutrition during pregnancy impacts offspring's epigenetic status-evidence from human and animal studies. Nutr. Metab. Insights 8(Suppl. 1), 41-47 (2015).

58 Stock NM, Humphries K, Pourcain BS et al. Opportunities and challenges in establishing a cohort study: an example from cleft lip/palate research in the United Kingdom. Cleft Palate Craniofac. J. 53(3), 317-325 (2016).

59 El-Maarri O, Walier M, Behne F et al. Methylation at global LINE-1 repeats in human blood are affected by gender but not by age or natural hormone cycles. PLoS ONE 6(1), e16252 (2011).

60 Zhang FF, Cardarelli R, Carroll J et al. Significant differences in global genomic DNA methylation by gender and race/ethnicity in peripheral blood. Epigenetics 6(5), 623-629 (2011).

61 Chalitchagorn K, Shuangshoti S, Hourpai N et al. Distinctive pattern of LINE-1 methylation level in normal tissues and the association with carcinogenesis. Oncogene 23(54), 8841-8846 (2004). 
(

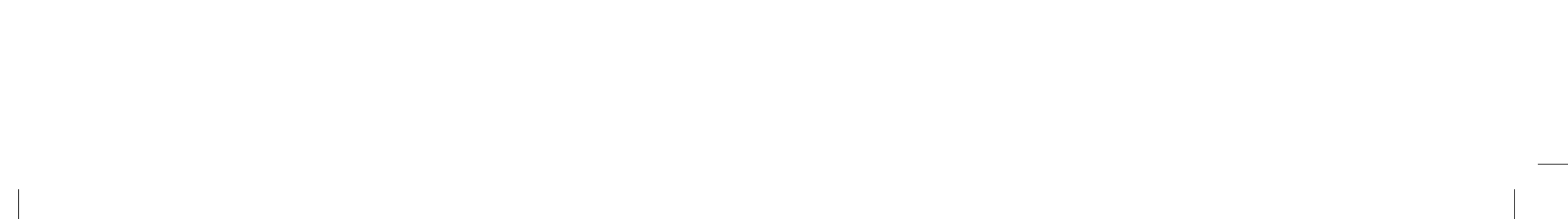

